\title{
Bootstrap inference for environmental trends
}

Citation for published version (APA):

Friedrich, M. (2020). Bootstrap inference for environmental trends. [Doctoral Thesis, Maastricht University]. Universitaire Pers Maastricht. https://doi.org/10.26481/dis.20201210mf

Document status and date:

Published: 01/01/2020

DOI:

10.26481/dis.20201210mf

Document Version:

Publisher's PDF, also known as Version of record

\section{Please check the document version of this publication:}

- A submitted manuscript is the version of the article upon submission and before peer-review. There can be important differences between the submitted version and the official published version of record.

People interested in the research are advised to contact the author for the final version of the publication, or visit the DOI to the publisher's website.

- The final author version and the galley proof are versions of the publication after peer review.

- The final published version features the final layout of the paper including the volume, issue and page numbers.

Link to publication

\footnotetext{
General rights rights.

- You may freely distribute the URL identifying the publication in the public portal. please follow below link for the End User Agreement:

www.umlib.nl/taverne-license

Take down policy

If you believe that this document breaches copyright please contact us at:

repository@maastrichtuniversity.nl

providing details and we will investigate your claim.
}

Copyright and moral rights for the publications made accessible in the public portal are retained by the authors and/or other copyright owners and it is a condition of accessing publications that users recognise and abide by the legal requirements associated with these

- Users may download and print one copy of any publication from the public portal for the purpose of private study or research.

- You may not further distribute the material or use it for any profit-making activity or commercial gain

If the publication is distributed under the terms of Article $25 \mathrm{fa}$ of the Dutch Copyright Act, indicated by the "Taverne" license above, 


\section{Bootstrap Inference for Environmental Trends}

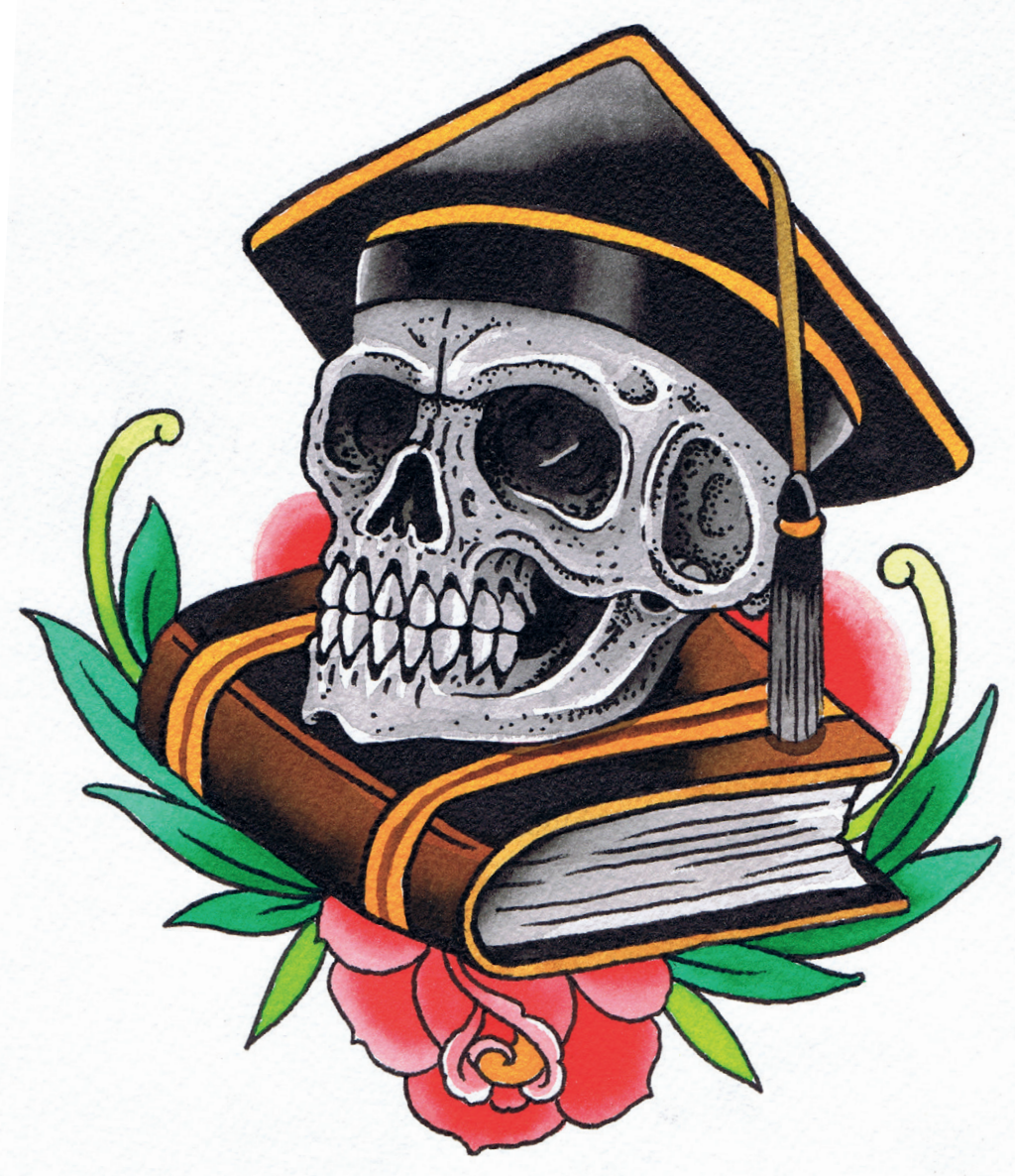

Marina Friedrich 



\section{Bootstrap Inference for Environmental Trends}


(C) Marina Friedrich, Maastricht 2020

All rights reserved. No part of this publication may be reproduced, stored in a retrieval system, or transmitted in any form, or by any means, electronic, mechanical, photocopying, recording or otherwise, without the prior permission in writing from the author.

This book was typeset by the author using $\mathrm{AT}_{\mathrm{E}} \mathrm{X}$.

Published by Universitaire Pers Maastricht ISBN: 978-94-6380-821-7

Printed in The Netherlands by Datawyse Maastricht 


\title{
Bootstrap Inference for Environmental Trends
}

\author{
DISSERTATION \\ to obtain the degree of Doctor at \\ Maastricht University, \\ on the authority of the Rector Magnificus, \\ Prof. dr. Rianne M. Letschert, \\ in accordance with the decision of the Board of Deans, \\ to be defended in public \\ on 10 December 2020, at 10.00 hours \\ by \\ Marina Friedrich
}




\section{Supervisors}

Prof. dr. J.R.Y.J. Urbain (deceased on 1 October, 2016)

Dr. S.J.M. Smeekes

Dr. E.A. Beutner

Prof. dr. F.C. Palm

\section{Assessment Committee}

Prof. dr. A.W. Hecq (Chair)

Dr. I. Wilms

Prof. dr. E. Hillebrand (Aarhus University)

Prof. dr. T. Proietti (University of Rome - Tor Vergata) 
To Jean-Pierre. 



\section{Acknowledgements}

In September 2014, I moved into my new office at Maastricht University. After having written my Bachelor and Master theses under the supervision of Stephan Smeekes and Jean-Pierre Urbain, I had signed a contract for three years as a Ph.D. student. During my studies, I needed quite some convincing that this was a good idea, but luckily, Jean-Pierre was very persuasive. Since I really enjoyed the previous thesis writing experience with Stephan and Jean-Pierre, I thought why not give it a try, it is probably just like writing a very big master thesis under their supervision. Now, roughly 5 years and 5 months later, I am writing this text as a different person.

These past years have been quite a journey and, oddly, the thought that I will at some point be able to write these acknowledgments (which certainly will be the part of my thesis read by the largest number of people) motivated me from a far distance. I did not allow myself to write this before finishing every other aspect of the thesis and I thought, I will really enjoy writing it. Now, I find it to be much harder than expected.

But let's give this a try. In the past years, there were many people who helped me finish this thesis and they are definitely too many to mention. I hope that nobody will be offended by not reading their name here. I do not intend to provide an exhaustive list of people, I rather want to thank some people in particular. In case someone does feel offended or left out, I apologize and as an excuse, I blame it on the long time period that it took me to finish, which makes it harder to keep track of such things. 
I would first like to thank the members of the reading committee, Ines Wilms, Alain Hecq, Tommaso Proietti and Eric Hillebrand, for agreeing to be part of this process and for taking the time to carefully read my thesis. I would also like to thank my former colleagues at Maastricht University. In particular, Karin van den Boorn and Yolanda Paulissen for being extremely supportive with all kinds of administrative issues. You were not only more than eager to help but also always up for a nice conversation. Most importantly, you never gave me the feeling of coming to your office at an inappropriate time. After having worked at a German bank where people you approach with any kind of questions will most likely make you feel unwelcome, this was a great experience.

I am extremely grateful that this time made it possible for me to meet Sean Telg, Yuliya Shapovalova, Anne Balter, Rasmus Lönn, Alexander Heinemann, Verena Jung, Aida Abiad, Hanno Reuvers, Yicong Lin and Andrey Kateshov. Thank you for great times at various coffee breaks, lunch breaks, conferences, (department) dinners and events, and for allowing me to hang out in your offices and complain.

Sean, although we met in the first year of the Bachelor studies 11(!) years ago, we only really got to know each other while sharing an office. Without you, this time would have been way less fun, less intense and less enjoyable (however, probably accompanied by more pleasant music). Thank you for your support and for letting me get to know you so well. Yuliya, you inspire me with your drive and motivation, independence as well as your passion for research and for your multifaceted hobbies. Anne, spending time with you is always a lot of fun. I think we have so many things in common and share similar views on life. Thank you for the great conversations. The same thing I want to say to you, Rasmus. I enjoyed all the conversations and our countless coffee breaks. I knew I could always come to your office, which is probably the door I knocked on the most, and you were always there to talk. Thank you for your openness and your support. Alex, Aida, Verena, thank you for all the coffee breaks and countless games of pool. Those were fun times.

Over the last 5 years, I had the chance of becoming part of the Climate Econometrics community and to spend a few weeks at the Climate Econometrics Network at Oxford, for which I would like to express my gratitude to Sir David Hendry, Felix Pretis, Luke Jackson, Andrew Martinez and Angela Wenham. I really enjoy being part of this network and I am looking forward to more conferences and future exchange. 
I would also like to thank my current colleagues at the Potsdam Institute for Climate Impact Research: Michael Pahle, Christian Gambardella, Eva-Maria Mauer, Samuel Okullo, Sebastian Osorio and Oliver Tietjen. Thank you for making me feel welcome in your group and for treating me like more of an expert on econometrics than I really am. Thank you also for enduring my constant complaints about my unfinished $\mathrm{PhD}$ thesis and for being excited with me about the final submission of my thesis and my first publication. My time at PIK made me find my way back to econometrics and I am excited to start my new position at VU Amsterdam in a few months.

Next, I would like to thank my parents, Gustel and Heinz-Georg Friedrich, for their constant and unconditional support. I know I am always welcome at your place and I can always reach out to you at any time. Thank you for making it possible for me to pursue my studies which ultimately led to this thesis. Additionally, I want to thank my aunt, Ellen Teeuwen-Strasser, and her family for many great conversations over red wine and tasty food.

Christian, thank you for being there for me in the past four years and for joining me to Berlin. Without you, I probably still wouldn't have completed this thesis. Thank you for being patient with me and for the invaluable help solving programming and Latex issues.

Finally and most importantly, I would like to finish these acknowledgments by thanking my four supervisors. First, I want to express my sincere gratitude to Franz Palm, for taking over the role of promotor after Jean-Pierre's unexpected passing. Thank you for encouraging me to finish and for believing in my research and its relevance. Second, I am thankful for the kind support of Eric A. Beutner. Although I am a bit disappointed that you never revealed to me what the A. in your name stands for, it was fun trying to find out. By now, I have collected "Approved", "Available", "Agree", "Applied" and "Awesome". To be continued.

Third, I owe more than a huge thank you to Stephan Smeekes. It has been an honor being your first PhD student. You have the extraordinary ability to understand the most complex issues and to be able to explain them in a very intuitive manner. You combine this with patience and kindness which makes you an amazing teacher, supervisor and person. You have taught me that our work often requires a certain creativity and that you cannot force ideas to come to you. This is valuable advice I often remind myself of. I would have probably never figured this out by myself. Your colleagues and future $\mathrm{PhD}$ students can consider 
themselves extremely lucky. You are the perfect supervisor. Your achievements are an inspiration.

Fourth, I would like to dedicate this thesis to Jean-Pierre Urbain. Without you, I would have never considered doing a PhD. Without you, I would not have gained so much confidence throughout the process. You were one of the kindest and most genuine people I have ever met. You were so passionate about econometrics and your hobbies. You genuinely cared about the people you worked with to an extend that I have never seen before and I have never seen since.

You took two hours from your very busy schedule just to give me travel advice for Chicago; you brought me your US sim card, maps and guide books to take on my trip; you offered me your DSLR camera to try out. Once, when I was worried about my upcoming yearly evaluation, you spontaneously took care of it right away, a week before the evaluation meeting was scheduled, so that I won't have to worry about it a second longer. You gave me constant feedback about my academic but also my personal development. You were one of the only people who saw from the beginning what a positive influence getting tattooed has on my confidence and you took every opportunity to express this. You once told me that it's more important that I am happy as a person than that I am functioning as an employee. All this meant so much to me.

I cannot express how much of a positive, long-lasting impact you had on my personal development by being an excellent supervisor and great human being. Our meetings have always made me feel happy and positive. Even when I was frustrated about my academic progress at times, I knew that everything will work out because of your support.

I will never forget the nerdy econometrics tattoo suggestions Jean-Pierre and Stephan had for me. This is why the cover of this thesis displays a traditional academic tattoo design which, as I told you, is the only academic tattoo I would ever get. Big thanks go out to Andreas Coenen for kindly agreeing to design this for me. I got a similar design tattooed on me a few months after Jean-Pierre passed away as a tattoo in his memory (which means that I have an econometrics tattoo after all). I had the great privilege of knowing him and I will forever be grateful.

Marina Friedrich 


\section{Contents}

1 Introduction 1

1.1 Nonparametric estimation - an overview . . . . . . . . . . . 3

1.2 Confidence intervals . . . . . . . . . . . . . . . . . . 9

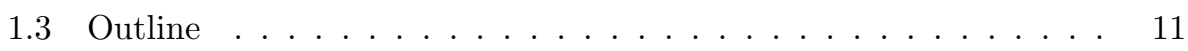

2 Autoregressive Wild Bootstrap Inference for Nonparametric $\begin{array}{ll}\text { Trends } & 13\end{array}$

2.1 Introduction . . . . . . . . . . . . . . . . . . 15

2.2 Trend Model with Missing Data . . . . . . . . . . . . . 17

2.3 Inference on Trends . . . . . . . . . . . . . . . . 19

2.3.1 Estimation of the Trend Function . . . . . . . . . . 20

2.3.2 Autoregressive Wild Bootstrap . . . . . . . . . . . . 22

2.3.3 Bootstrap Confidence Bands . . . . . . . . . . . . 26

2.4 Asymptotic Theory . . . . . . . . . . . . . . . . . 28

2.5 Simulation Study . . . . . . . . . . . . . . . . 32

2.5.1 Simulation Setup . . . . . . . . . . . . 32

2.5.2 Simulation Results . . . . . . . . . . . . . . . . 35

2.6 Trends in Atmospheric Ethane . . . . . . . . . . . . . . . . . 40

2.7 Conclusion ....................... . . . 47

2.A Technical Results . . . . . . . . . . . . . . 49

2.A.1 Auxiliary lemmas . . . . . . . . . . . . . 49

2.A.2 Pointwise Results . . . . . . . . . . . . . . . 51

2.A.3 Uniform Results . . . . . . . . . . . . . . . 67

2.B Additional Proofs . . . . . . . . . . . . . . . . 70 
2.C Additional simulation results . . . . . . . . . . . . . . . 85

2.C.1 Homoskedastic DGPs . . . . . . . . . . . . . . 85

2.C.2 Varying $a$ and $k$ in the heteroskedastic DGPs . . . . . . . 89

2.C.3 Missing data - varying the bandwidth . . . . . . . . . . 117

2.D Additional graphs for varying the number of Fourier terms . . . . . 121

3 Trends in atmospheric time series: an application to ethane $\quad \mathbf{1 2 7}$

3.1 Introduction . . . . . . . . . . . . . . . . . . . . . . . . 129

3.2 The ethane data . . . . . . . . . . . . . . . . . . . . . 131

3.3 The linear trend approach . . . . . . . . . . . . . . . . . 132

3.3.1 A broken trend model . . . . . . . . . . . . . . . . . 132

3.3 .2 Estimation .................... 134

3.3.3 Confidence intervals . . . . . . . . . . . . . . . 135

3.3.4 Data application . . . . . . . . . . . . . 137

3.4 A smooth trend function . . . . . . . . . . . . . . . . 139

3.4.1 The nonparametric trend model . . . . . . . . . . . 139

3.4.2 Inference on trend shapes . . . . . . . . . . . . . . . 147

3.5 Conclusion ...................... 154

3.A Additional results . . . . . . . . . . . . . . . . . . 155

3.B Simulation study . . . . . . . . . . . . . . . . . . . 158

4 Bootstrap Simultaneous Confidence Bands For Time-varying Coefficient Models $\quad 163$

4.1 Introduction . . . . . . . . . . . . . . . . . . . . 165

4.2 The model . . . . . . . . . . . . . . . . . . . . 167

4.3 Nonparametric estimation . . . . . . . . . . . . . . . 168

4.3.1 The local linear estimator . . . . . . . . . . . . . . . 168

4.3 .2 Bandwidth selection . . . . . . . . . . . . 170

4.4 Confidence intervals with the sieve (wild) bootstrap . . . . . . . . . . . . . . . . . . 171

4.4.1 Bootstrap algorithms . . . . . . . . . . . . 172

4.4.2 Constructing confidence intervals . . . . . . . . . . . 175

4.5 Simulation study . . . . . . . . . . . . . . . 176

4.5.1 The data generating process . . . . . . . . . . . . 177

4.5.2 The ARMA $(1,1)$ specification . . . . . . . . . . . . 179

4.5.3 The $\operatorname{GARCH}(1,1)$ specification . . . . . . . . . . . 187

4.5.4 Bandwidth selection methods . . . . . . . . . . . 189 
4.6 Application to the EU Emissions Trading Scheme ........................ 193

4.6.1 Background and related literature . . . . . . . . . 193

4.6 .2 The data . . . . . . . . . . . . . . . 196

4.6.3 Empirical results ................. 200

4.7 Conclusion .......................... 208

4.A Additional simulation results . . . . . . . . . . . . . . 209

4.A.1 Additions to the main simulation study . . . . . . . . . . 210

4.A.2 Time-varying CAPM models . . . . . . . . . . . . . 212

4.B Additional empirical results . . . . . . . . . . . . . . . . . . 214

$\begin{array}{llr}5 & \text { Conclusion } & 227\end{array}$

$\begin{array}{llr}6 & \text { Valorization } & 231\end{array}$

$\begin{array}{ll}\text { Bibliography } & 233\end{array}$

$\begin{array}{ll}\text { Nederlandse Samenvatting } & 243\end{array}$

$\begin{array}{lr}\text { Curriculum Vitae } & 247\end{array}$ 



\section{Chapter 1}

\section{Introduction}

The usefulness of econometric and statistical tools goes far beyond classical economic and financial applications. Facing the harmful consequences of climate change, there is an increasing urgency in climate policy and environmental applications are of growing importance. Like economic variables, environmental time series and the relationships between them are constantly evolving. In such applications, it is common that due to physical constraints, structural change does not happen abruptly but gradually over time. The diverse nature of these changes make statistical modeling challenging. It is the goal of this thesis to provide a collection of methods which provide reliable inference in the presence of gradual structural change. The main focus lies on the development and illustration of tools which can be used in environmental applications.

If we believe, for instance, that the true underlying structure of the quantities of interest is of a complex form, it is important to have flexible methods available as a basis for our modeling. One group of flexible models are time-varying coefficient models. These models can be formalized both parametrically and nonparametrically. The major difference is that nonparametric models do not rely on a specification of the form of the change in advance - an advantage on which we will elaborate further in the next section.

While the estimation of these models is well-established in the literature, conducting inference is not complete without the construction of confidence intervals which is an active area of research in econometrics. One purpose of this thesis is to develop bootstrap methods which are able to provide reliable inference on nonparametric time-varying coefficient models. In particular, the new bootstrap 
methods can be used to obtain pointwise confidence intervals as well as simultaneous confidence bands around nonparametrically estimated parameter curves.

Bootstrap methods are known to have desirable small sample performance and therefore are of great practical relevance, which goes beyond the analysis of economic relationships. As mentioned above, environmental applications are another field of research where this thesis has added value. An additional challenge which often arises in these applications are missing observations. Recording observations of such time series often involves complex instruments and advanced measurement facilities. In addition, certain measurement conditions have to be met to guarantee a smooth recording process. In such situations, instrument failure as well as unfavorable measurement conditions can lead to a severe missing data problem.

This thesis starts off in Chapter 2 with a pure deterministic trend model in which the trend is represented by a smooth function of time. The setting is a general one since it allows for heteroskedastic as well as correlated errors. To obtain confidence intervals around the nonparametric trend estimate, the bootstrap method of our choice is a modification of the wild bootstrap - the autoregressive wild bootstrap. Using this approach, we analyze time series of atmospheric ethane which is in itself an additional purpose of this thesis.

Measurements of atmospheric ethane suffer from the aforementioned missing data problem. The autoregressive wild bootstrap has a natural way of handling missing data, which is demonstrated both in the underlying theory of Chapter 2 and when we apply it to the atmospheric ethane time series. In Chapter 3, a thorough analysis of the series is performed. The goal is to establish an upward trend present in atmospheric ethane over the last few years. A working hypothesis in the field of geophysics is that this increase can be attributed to an increased activity in shale gas extraction. We aim at providing additional evidence for this hypothesis in Chapter 3, which is robust to the choice of modeling.

In some applications, the pure trend model is too restrictive, which is why we extend it to include exogenous regressors in Chapter 4. This adds a lot of flexibility and allows the researcher to analyze more complex relationships. For this extension, we propose a sieve bootstrap approach to construct confidence intervals for the time-varying parameter curves. We use our approach to study an important topic of environmental economics: the relationship between allowance prices and their most important price drivers in the world's largest cap-and-trade market - the European Emissions Trading Scheme. 
Although the three main chapters of this thesis - Chapter 2, 3 and 4 - have some commonalities, they represent independent work. We prefer to keep them self-contained such that they can be read in arbitrary order or as an individual piece of work. To this end, consecutive chapters do not rely on notation that was introduced in previous chapters. This comes at the cost that some concepts, methods or algorithms appear in multiple chapters.

To set the ground, this chapter provides an overview of nonparametric estimation and bootstrapping in the following two sections. In Section 1.1, we present the basic idea behind nonparametric estimation techniques in the context of regression estimation combined with a brief summary of the related literature. In Section 1.1, we introduce bootstrapping for the construction of confidence intervals and refer to a selection of previous work on this topic.

\subsection{Nonparametric estimation - an overview}

The need to deviate from constant coefficient models in economic time series and the search for more flexible tools of econometric modeling became apparent in the early work by Chow (1960) with his break point analysis, which assumes a known breakpoint. Various extensions followed, where the date of the breakpoint does not necessarily need to be known in advance. A few years later, deterministically time-varying coefficient models were introduced as a response to the observation that changes usually do not appear abruptly but smoothly over time. The earliest work on this topic dates back to 1965, when Priestley (1965) introduced processes with slowly changing spectral densities. Robinson (1989) extends the idea to a linear regression set-up and to nonlinear regression in Robinson (1991). More recent work on this topic includes Orbe et al. (2005), Orbe et al. (2006) and Kapetanios (2008). They all use a variant of the nonparametric, time-varying coefficient model, which is used in this thesis and goes back to Cai (2007), but they apply a different estimation procedure. The model presented in Cai (2007) is the most general one among the aforementioned.

This development from constant to time-varying coefficient models shows that nonparametric modeling with its apparent advantages plays a prominent role in the literature. In contrast to parametric estimation, it is extremely flexible and robust to functional form misspecification. In a parametric setting, incorrectly assuming a stable relation while the true structure is varying over time leads to adverse effects. Inconsistency of the estimates is a likely result. In nonparametric 
modeling, no prior information on the functional form of the coefficient curves is needed. Additionally, with the help of nonparametric estimation, the researcher can get an idea about the functional form of the economic relation, which can then be used for further parametric fitting. In a situation of smooth structural changes it is therefore more appealing to use nonparametric models.

A related and important feature of economic as well as climate time series are deterministic trends. They are a crucial source of fundamental change and although they are so frequently observed, modeling them is a challenging task. As pointed out by Phillips (2001) in his review on trending time series, there is little guidance from economic theory about how to model the trends. The same holds true for climate time series, thinking about the case of temperature data, for example. If we set up a parametric model and use a certain trend specification, we implicitly make the assumption that the true process is included in our model. This assumption is crucial, because misspecification can result in inconsistent parameter estimates. Trend estimation therefore is an example where it is more appropriate to rely on nonparametric estimation when there is doubt about the true shape of the trend. This is the topic of Chapter 2.

In modeling economic growth, for example, traditional models as in Boswijk and Franses (2006) usually specify a certain form, mostly linear, between a set of regressors and a country's growth rate. Assuming this specific form, however, does not allow to fully explore the complex structure of the data. In growth analysis, it is particularly reasonable to expect that the relation between economic growth and the explanatory variables is subject to structural changes over time. In such a setting, it is more adequate to express each coefficient as a function of time. This can be achieved using time-varying coefficient models which are discussed in Chapter 4.

In the econometric literature, there are several different approaches to nonparametric modeling. While a general overview over existing methods is given in Fan and Gijbels (1996), the focus for the rest of this section lies on the introduction of two different classes of local models: local constant and local polynomial fitting. We now give the basic idea of nonparametric estimation and as an illustration we use the simple case of a deterministic trend model. The introduction is based on the books by Fan and Gijbels (1996) and Hansen (2009).

We start by considering the linear trend model, for $y_{t}$ with $t=1, \ldots, n$,

$$
y_{t}=\alpha+\beta t+\epsilon_{t}
$$


It predicts a linear development of $y$ over time. Denoting the regression function by $m(\cdot)$, we obtain:

$$
\mathbb{E}\left(y_{t} \mid t\right) \equiv m_{t}=\alpha+\beta t
$$

As a result, estimating a linear trend model implicitly assumes that the trend function can be best described by a linear shape. While this might work well in some situations, it can be too restrictive in others. In those situations, the researcher can benefit from using nonparametric regression. The main idea behind nonparametric regression is to relax the linearity assumption and to let the data determine the form of the trend function. Obviously, there are other approaches, which are less restrictive than linear trends. One example is a parametric trend of a polynomial type, where the form of the trend is assumed to be a finite order polynomial. Although this is a much more general approach, the major drawback mentioned above remains: the overall shape of the trend in any parametric model has to be specified in advance. This disadvantage is resolved using nonparametric techniques.

In contrast to the conditional expectation function (1.2) originating from linear trend regression, in a general nonparametric model, the form of the function $m(\cdot)$ is not specified. All that is known is that the trend is a smooth function of time, which can be linear, quadratic, polynomial or of any other form. This means that $m(\cdot)$ comes from the class of smooth functions, which is of infinite dimension. To illustrate, a general nonparametric model can be stated as

$$
y_{t}=m_{t}+\epsilon_{t}
$$

As is common in this literature, we need to specify $m_{t}$ as $m_{t}=m(t / n)$ such that the trend function is dependent on the sample size $n$ through the so-called intensity assumption. It is a function on the unit interval: $m:[0,1] \rightarrow \mathbb{R}$. Intuitively, as explained in Robinson (1989), this assumption expresses the need to increase the amount of local information on which the estimator depends and it is crucial to obtain consistency. In other words, it means that the support of the estimator needs to become more dense as $n$ increases, which would not be the case in this framework, if this assumption did not exist, since the support is the natural numbers.

When it comes to the estimation of nonparametric models, a popular approach is local modeling. We start by explaining the general principle of this approach 
by means of introducing local constant modeling. Subsequently, on the basis of this knowledge, we illustrate local linear and local polynomial fitting. In local constant modeling, for a given time point $\tau$, the trend function is estimated by a constant for a fraction of the data around the point $\tau$. This is done for all $\tau$ to obtain a smooth trend curve. Similarly, for local polynomial modeling, the same basic idea applies, but instead of locally approximating the trend function in the neighborhood around $\tau$ by a constant, a polynomial approximation is used. The local linear approach is part of the more general class of local polynomial regressions with a polynomial of order one.

In an $h$-neighborhood around $\tau$ the local constant model reads:

$$
y_{t}=m(\tau)+\epsilon_{t} \quad \text { for } \quad t / n \in[\tau-h, \tau+h] .
$$

Another way of formalizing this approach is by a weighted least squares problem using a weighting function $K(\cdot)$ :

$$
\sum_{t=1}^{n}\left\{y_{t}-m(\tau)\right\}^{2} K\left(\frac{t / n-\tau}{h}\right)
$$

The condition that $t / n$ has to be in an $h$-neighborhood around the point $\tau$ is absorbed into the weighting function $K(\cdot)$, which is called kernel function. It is a non-negative unimodal function, which usually embodies an indicator function of the form $\mathbb{1}(|\cdot| \leq 1)$ to make sure that it has compact support. In addition, a kernel function $K(u)$ usually satisfies $0 \leq K(u) \leq \infty, K(u)=K(-u), \int_{-\infty}^{\infty} K(u) d u=1$ and $\int_{-\infty}^{\infty} u^{2} K(u) d u<\infty$.

The intuitive idea behind this approach is to take locally weighted averages by fitting a constant line to the data in a neighborhood of a point $\tau$. Finding the value of $m(\tau)$, for which the sum of squares in (1.5) is minimized, results in the famous Nadaraya-Watson estimator (compare Nadaraya (1964) and Watson (1964)). In more general terms, the local constant approximation can be written as

$$
\hat{m}(\tau)=\underset{m(\tau)}{\operatorname{argmin}} \sum_{t=1}^{n}\left\{y_{t}-m(\tau)\right\}^{2} w_{t}(\tau)=\frac{\sum_{t} w_{t}(\tau) y_{t}}{\sum_{t} w_{t}(\tau)},
$$


where the weights $w_{t}(\tau)$ can take different forms for different estimators. For the Nadaraya-Watson estimator the form of $w_{t}(\tau)$ is

$$
w_{t}(\tau)=K\left(\frac{t / n-\tau}{h}\right) .
$$

The above discussion explains the general idea of local modeling using the example of a local constant approximation. Local linear and local polynomial fitting are easily derived from here.

For the local linear model, consider, similar to the weighted least squares problem given in (1.5), a weighted regression of $y_{t}$ on an intercept and a slope. The resulting least squares problem with kernel function $K(\cdot)$ reads:

$$
\sum_{t=1}^{n}\left\{y_{t}-m(\tau)-m^{(1)}(\tau)(t / n-\tau)\right\}^{2} K\left(\frac{t / n-\tau}{h}\right)
$$

with $m^{(j)}(\cdot)$ denoting the $j$-th derivative of $m(\cdot) .{ }^{1}$ Here, the idea is to fit a locally weighted linear regression to the data in a neighborhood of a point $\tau$. Finding the values of $m(\tau)$ and $m^{(1)}(\tau)$, for which this sum of squares is minimized, results in the local linear estimator. It has an explicit solution, which provides us with an analytic expression for the local linear estimators $\hat{m}(\tau)$ and $\hat{m}^{(1)}(\tau)$. For notational convenience set, for each $\tau$,

$$
z_{t}(\tau)=\left(\begin{array}{c}
1 \\
t / n-\tau
\end{array}\right)
$$

and

$$
K_{t}(\tau)=K\left(\frac{t / n-\tau}{h}\right)
$$

Then, the solution to the above minimization problem is given by

$$
\left(\begin{array}{c}
\hat{m}(\tau) \\
\hat{m}^{(1)}(\tau)
\end{array}\right)=\left(\sum_{t=1}^{n} K_{t}(\tau) z_{t}(\tau) z_{t}(\tau)^{\prime}\right)^{-1}\left(\sum_{t=1}^{n} K_{t}(\tau) z_{t}(\tau) y_{t}\right)
$$

\footnotetext{
${ }^{1}$ For $j=0$, we obtain the original function $m^{(0)}(\cdot)=m(\cdot)$.
} 
The idea of the local polynomial estimator is closely linked to the concept of Taylor approximation. It can be seen as locally approximating the trend function $m(\cdot)$ by a Taylor approximation. Applying a $p$-th order Taylor expansion to the function $m(\cdot)$, we obtain for $t / n$ in a neighborhood of $\tau$ :

$$
m(t / n) \approx \sum_{j=0}^{p} \frac{m^{(j)}(\tau)}{j !}(t / n-\tau)^{j} \equiv \sum_{j=0}^{p} \mu_{j}(\tau)(t / n-\tau)^{j}
$$

This suggests that, following the same approach as in the derivation of equations (1.5) and (1.8), we can use the following locally weighted polynomial regression:

$$
\sum_{t=1}^{n}\left\{y_{t}-\sum_{j=0}^{p} \mu_{j}(\tau)(t / n-\tau)^{j}\right\}^{2} K\left(\frac{t / n-\tau}{h}\right) .
$$

Minimizing this expression gives us $\hat{\mu}_{j}(\tau)$, for $j=0, \ldots, p$. For $p=1$, we obtain the local linear regression as in (1.8).

Comparing equation (1.11) with the above Taylor approximation (1.10) thus suggests the following estimator of $m^{(j)}(\tau)$, for $j=0, \ldots, p$ :

$$
\hat{m}^{(j)}(\tau)=j ! \hat{\mu}_{j}(\tau)
$$

The parameter $h$ which determines the width of the neighborhood around $\tau$ is called bandwidth. Its choice is a crucial aspect of any local fitting method as it simultaneously controls the amount of smoothing and the complexity of the estimation. A small value of the bandwidth stands, on one hand, for a small approximation error and a resulting small modeling bias. On the other hand, it means that only a few data points are included in the local neighborhood and therefore, the variance of the estimator is large. In addition, the estimated curve is less smooth and the model is more complex than with a larger bandwidth.

In contrast, a large value of the bandwidth can create a large modeling bias, but the model is less complex and the amount of smoothing will be large. As $h \rightarrow \infty$, the estimator approaches the linear regression estimator because in the limiting case all observations receive equal weight. For $h \rightarrow 0$, the procedure would result in linear interpolation of the data points. Therefore, bandwidth selection controls the complexity of the model and in addition, there is a bias-variance tradeoff. 
Comparing parametric and nonparametric fitting, the bandwidth parameter can be seen as an additional dimension. Parametric fitting is like nonparametric fitting, where the choice of the bandwidth parameter is constant and different families of models are considered. Taking an infinite bandwidth is unquestioned in all situations of parametric modeling, while with nonparametric estimation, the bandwidth is seen as an additional parameter, which is carefully chosen so that the estimation outcome fits the data well. Throughout the chapters of this thesis, we will encounter a collection of different (data-driven) ways to select this important parameter.

\subsection{Confidence intervals}

Estimating the trend, or more general, the parameter curves is an important first step to arrive at reasonable conclusions about the form of the investigated relation. It is, nevertheless, of limited use without the next step, which is to assess the uncertainty around the estimates. In this thesis we advocate the use of bootstrap methods for this purpose. We will go into detail about the specific methods in each chapter. Here, we merely provide a general introduction.

The classical bootstrap approach relies on taking (artificial) random resamples of the data. It replicates the estimation step using each resample and derives the confidence intervals from the distribution of the replicated estimates. Formally, the idea of the original bootstrap can be introduced as follows. Consider a time series $\left\{y_{t}\right\}_{t=1}^{n}$ which is a random sample drawn from $F$. We are interested in a specific parameter $\theta$. Denote by $\hat{\theta}_{n}$ an estimator like the local constant estimator introduced in the previous section. We can write the estimator applied to the time series $y_{t}$ as

$$
\hat{\theta}_{n}=\hat{\theta}_{n}\left(y_{1}, y_{2}, \ldots, y_{n}\right)
$$

The CDF of this estimator is

$$
G_{n}(u, F)=\mathbb{P}\left(\hat{\theta}_{n} \leq u \mid F\right)
$$

The CDF are dependent on the distribution $F$ which is unknown and needs to be approximated if we want to conduct inference. While asymptotic inference is based on letting $n$ grow such that $G_{n}(u, F)$ is approximated by $G(u, F)=$ $\lim _{n \rightarrow \infty} G_{n}(u, F)$, in the bootstrap approach $F$ is replaced by a consistent estimate 
$F_{n}$ such that we obtain

$$
G_{n}^{*}(u)=G_{n}\left(u, F_{n}\right)
$$

This is called the bootstrap distribution. This idea was first put forward by Efron (1979). When we draw random samples from this distribution, we obtain a bootstrap series $\left\{y_{t}^{*}\right\}_{t=1}^{n}$. Applying the estimator $\hat{\theta}_{n}$ to this series, we get

$$
\hat{\theta}_{n}^{*}=\hat{\theta}_{n}\left(y_{1}^{*}, y_{2}^{*}, \ldots, y_{n}^{*}, F_{n}\right)
$$

A popular choice for $F_{n}$ is the empirical distribution function (EDF) defined as

$$
F_{n}(y)=\frac{1}{n} \sum_{t=1}^{n} \mathbb{1}\left(y_{t} \leq y\right)
$$

It is a nonparametric estimate of $F$ which is, indeed, a consistent for $F$ (Hansen, 2009). Although it takes the form of a step function, it gets close to the CDF $F$ as $n$ gets large.

Since the computational burden to calculate $G_{n}^{*}$ directly is too large, Efron (1979) proposed to approximate it by Monte Carlo simulation. The following algorithm describes this procedure:

Algorithm 1.1 (Nonparametric Bootstrap).

1. Draw a random bootstrap sample from $F_{n}$ and denote it by $\left(y_{1}^{*}, y_{2}^{*}, \ldots, y_{n}^{*}\right)$.

2. Apply the estimator $\hat{\theta}_{n}$ to this sample and obtain $\hat{\theta}_{n}^{*}=\hat{\theta}_{n}\left(y_{1}^{*}, y_{2}^{*}, \ldots, y_{n}^{*}, F_{n}\right)$.

3. Repeat Steps 2 and $3 B$ times. The collection of bootstrap estimates forms the approximation of the bootstrap distribution.

When $F_{n}$ is the EDF as described above, this algorithm is called nonparametric bootstrap and it is the most commonly used version. In this case, Step 1 becomes equivalent to drawing randomly and with replacement from the data $\left\{y_{t}\right\}_{t=1}^{n}$. There exists a parametric counterpart. In the parametric bootstrap, the assumption is made that $F$ comes from a certain parametric family $F(u \mid \vartheta)$. Then, we estimate $\vartheta$ from the original data as $\hat{\vartheta}$ and plug this estimate into $F(u \mid \vartheta)$ such that we obtain $F(u \mid \hat{\vartheta})$. Using this for $F_{n}$ in Step 1 of the above algorithm defines the parametric bootstrap. Thus, it is the sampling step that makes the difference between the parametric and the nonparametric bootstrap. Compared to inference 
based on asymptotic theory, bootstrap methods are known to be more robust to misspecification and have a better overall small sample performace.

The nonparametric version of bootstrap presented here is often called i.i.d. bootstrap, as it relies on the assumption that $\left(y_{1}, y_{2}, \ldots, y_{n}\right)$ is a random sample. Therefore, it was originally designed to deal with data without serial dependence. It needs to be modified in a time series set-up to draw resamples, which preserve the dependence that is present in the data. There are several modifications of the classical bootstrap that are able to handle heteroskedasticity as well as serial correlation. A wild bootstrap approach, originally proposed by $\mathrm{Wu}$ (1986), is designed for the context of heteroskedasticity. Both a block bootstrap, dating back to Künsch (1989), and a sieve bootstrap invented by Kreiss (1992) and Bühlmann (1997) have been developed for serially correlated time series data. Many more time series bootstrap methods exist, but not all are applicable to nonparametric estimation.

For nonparametric trend estimation, a sieve bootstrap method is proposed by Bühlmann (1998). Further research on bootstrap methods for nonparametric trend estimation is presented in Neumann and Polzehl (1998) and Hidalgo (2005). Neumann and Polzehl (1998) use a wild bootstrap method to obtain simultaneous confidence bands for a local polynomial estimate of a trend function, while the proposed bootstrap method in the paper by Hidalgo (2005) relies on bootstrapping in the frequency domain instead of the time domain. A final paper to be mentioned in this context is Kapetanios (2008), who develops a statistical test to decide between constant and time-varying coefficient models.

\subsection{Outline}

The remainder of this thesis is structured as follows. In the next chapter, Chapter 2 , an autoregressive wild bootstrap method is presented in the context of nonparametric trend estimation. The method can be used to construct pointwise confidence intervals as well as simultaneous confidence bands around the trend estimate. We derive the asymptotic distribution of the nonparametric estimator in the presence of heteroskedasticity, serial correlation as well as missing data. We show the asymptotic validity of the bootstrap method. A simulation study investigates the small sample performance.

In Chapter 3, we study trends in atmospheric ethane. For this purpose, we present various methods to analyze trends in atmospheric time series. These in- 
clude both parametric methods as well as the nonparametric approach presented in Chapter 2. This application constitutes a relevant topic in the field of geophysics and at the same time, it serves as an illustration and motivation for our previously proposed method. For the parametric part, we first use linear trend modeling with a break as in Bai and Perron (1998) where the location of the break is unknown. We provide confidence intervals around the break location using a version of the autoregressive wild bootstrap. Second, we propose two tests to investigate the behavior of the trend after the break.

The goal of Chapter 4 is to extend the nonparametric trend model of Chapter 2 in such a way that we can include a set of exogenous regressors in addition to the nonparametric trend. We propose a flexible local linear nonparametric estimator to estimate the trend function and the coefficient curves. This we combine with a sieve bootstrap method to obtain confidence intervals. Similar as in Chapter 2, we suggest a simple procedure to convert the pointwise confidence intervals into simultaneous confidence bands. We investigate the performance of the proposed approach in an extensive simulation study. The final part of the chapter shows the relevance of the method to a popular topic of environmental economics.

Chapter 5 provides an overall conclusion of the thesis, while the respective conclusions of the individual topics can be found at the end of each chapter. The conclusion is followed by a valorization chapter, Chapter 6 , which elaborates on the relevance of the presented topics for society. This thesis ends with the references and a short curriculum vitae of the author. 


\section{Chapter 2}

\section{Autoregressive Wild}

\section{Bootstrap Inference for}

\section{Nonparametric Trends}

In this chapter, we propose an autoregressive wild bootstrap method to construct confidence bands around a smooth deterministic trend. The bootstrap method is easy to implement and does not require any adjustments in the presence of missing data, which makes it particularly suitable for climatological applications. We establish the asymptotic validity of the bootstrap method for both pointwise and simultaneous confidence bands under general conditions, allowing for general patterns of missing data, serial dependence and heteroskedasticity. The finite sample properties of the method are studied in a simulation study. We use the method to study the evolution of trends in daily measurements of atmospheric ethane obtained from a weather station in the Swiss Alps, where the method can easily deal with the many missing observations due to adverse weather conditions. ${ }^{1}$

\footnotetext{
${ }^{1}$ This chapter is based on the article Autoregressive Wild Bootstrap Inference for Nonparametric Trends by Friedrich, M., Smeekes, S. and J.-P. Urbain (2020), Journal of Econometrics, 214,1 , p. 81-109.
} 



\subsection{Introduction}

The analysis of smoothly evolving trends is of interest in many fields such as economics and climatology. For instance, trend analysis in environmental variables is of major importance, as it can often directly be linked to climate change. Given that trends typically do not evolve in a linear way, fitting linear trends to the data does not uncover actual change accurately. Instead, one would like to use more flexible trend models that avoid making parametric assumptions on the form of the trend. A large body of statistical and econometric research therefore focuses on nonparametric trend modeling and estimation.

In addition, when modeling trends in temperature and emission data, researchers also need to take into account that serial dependence and heteroskedasticity may be present in the data, see for example Franses and Vogelsang (2005) and McKitrick and Vogelsang (2014) who study parametric trend modeling in temperature series in the presence of serial dependence. Bootstrap methods provide an easy and powerful way to account for heteroskedasticity and autocorrelation. Bühlmann (1998) shows the validity of the autoregressive sieve bootstrap for nonparametric trend modeling under general forms of dependence. Neumann (1998) uses a wild bootstrap method to achieve robustness to heteroskedasticity for a similar model.

The wild bootstrap approach is also suitable for dealing with missing data, as advocated for example by Shao (2010b). It does not require any resampling and therefore the missing data points can keep their original date in a bootstrap sample. In particular in climatology, this feature of the wild bootstrap offers an important benefit over other methods, since there is no need of imputing missing data points. Missing data are a prominent feature in many climatological datasets due to instrument failure or adverse weather and measurement conditions; for instance, in our application, data are missing when cloud cover prevents measurements from being taken. The wild bootstrap, however, relies on independence of the error terms, which is a situation rarely encountered in practice. To relax this strong assumption, dependent versions of wild bootstrap methods have been proposed - see Shao (2010b), Leucht and Neumann (2013) and Smeekes and Urbain (2014) - but not in the context of nonparametric trend estimation. Moreover, so far no theory exists on the validity of such wild bootstrap methods in the presence of serial dependence, heteroskedasticity and missing data. In this chapter, we ad- 
dress this issue and propose an autoregressive wild bootstrap method that provides valid inference under general conditions for nonparametric trend modeling.

Next to the basic pointwise confidence intervals, we also study simultaneous confidence bands, which are often more informative about trend shapes than pointwise confidence intervals. Research questions, like whether upward trends are present over a certain period of time, should be addressed with simultaneous confidence bands as they involve multiple points in time at once. Wu and Zhao (2007) derive such bands for the nonparametric trend model that have asymptotically correct coverage probabilities, but do not consider bootstrap methods. Bühlmann (1998) proposes sieve bootstrap-based simultaneous confidence bands that are not only asymptotically valid but also have good small sample performance. They can, however, not easily be adjusted to be applicable to time series with missing data, as the autoregressive sieve bootstrap requires imputation of the missing values through for instance the Kalman filter. While this is certainly possible, it complicates implementation. One can also argue about how accurate imputation methods are when a majority of the data are missing, as we face in our climatological application. Instead, we provide a much simpler alternative that requires no adjustments at all in the presence of missing data.

To illustrate our methodology, we study a time series of atmospheric ethane emissions for which almost $70 \%$ of the data points are missing. When weather conditions are unfavorable - in particular due to cloud cover - measurements cannot be taken. The series has previously been investigated by Franco et al. (2015). Atmospheric ethane is an indirect greenhouse gas which can be used as an indicator of atmospheric pollution and transport. It is emitted during shale gas extraction and since shale gas has become more and more important as a source of natural gas, nonparametric trend analysis in ethane data provides geophysicists and climatologists with a tool to link trend changes to shale gas extraction activities, as well as study long-term climatological change.

The chapter is organized as follows. In Section 2.2, our trend model is introduced along with the missing data generating mechanism. Section 2.3 describes the estimation procedure and the construction of bootstrap confidence bands. Subsequently, Section 2.4 derives the asymptotic properties of our method. Finite sample performance is analyzed in Section 2.5 in a simulation study. Trends in atmospheric ethane are studied in Section 2.6. Section 2.7 concludes. All technical details including proofs are given in Appendix A, while Supplementary Appendices $\mathrm{B}$ to $\mathrm{D}$ provide further results. 
Finally, a word on notation. We denote by $\stackrel{d}{\rightarrow}$ weak convergence and by $\stackrel{p}{\rightarrow}$ convergence in probability. Whenever a quantity has a subscript ${ }^{*}$, it denotes a bootstrap quantity, conditional on the original sample. For instance, bootstrap weak convergence in probability is denoted by ${\stackrel{d^{*}}{\longrightarrow}}_{p}$ (cf. Gine and Zinn, 1990). $\lfloor x\rfloor$ stands for the largest integer smaller than or equal to $x$. For any functions $f(x)$ and $g(x)$, defined on the same domain, $f^{(i)}(x)=\frac{d^{i}}{d x^{i}} f(x)$ and $[f g]^{(i)}(x)=\frac{d^{i}}{d x^{i}} f(x) g(x)$.

\subsection{Trend Model with Missing Data}

Consider the following data generating process (DGP):

$$
y_{t}=m\left(\frac{t}{n}\right)+z_{t} \quad t=1, \ldots, n,
$$

where $m(\cdot)$ is a smooth deterministic trend function and $z_{t}=\sigma_{t} u_{t}$ is a weakly dependent stochastic component. $\sigma_{t}$ captures unconditional heteroskedasticity and $\left\{u_{t}\right\}$ is a linear process

$$
u_{t}=\sum_{j=0}^{\infty} \psi_{j} \epsilon_{t-j}, \quad \psi_{0}=1
$$

with autocovariance function $R_{U}(k)=\mathbb{E} u_{t} u_{t+k}$ and long-run variance

$$
\Omega_{U}=\sum_{k=-\infty}^{\infty} \mathbb{E} u_{t} u_{t+k}=\sum_{k=-\infty}^{\infty} R_{U}(k)
$$

Not all observations $y_{1}, \ldots, y_{n}$ are observed in practice. For this purpose, define the process $\left\{D_{t}\right\}$ as an indicator for whether the observations at each time are observed:

$$
D_{t}=\left\{\begin{array}{ll}
1 & \text { if } y_{t} \text { is observed } \\
0 & \text { if } y_{t} \text { is missing }
\end{array} \quad t=1, \ldots, n\right.
$$

Assumptions 2.1 to 2.4 contain the formal conditions that $\left\{y_{t}\right\}$ and $\left\{D_{t}\right\}$ satisfy.

Assumption 2.1. $m:[0,1] \rightarrow \mathbb{R}$ is a twice continuously differentiable deterministic function on $(0,1)$ with $\sup _{0<\tau<1}\left|m^{(i)}(\tau)\right|<\infty$ for $i=0,1,2$.

Assumption 2.2. $\sigma:[0,1] \rightarrow \mathbb{R}^{+}$is a Lipschitz continuous deterministic function. 
Assumption 2.3. $\left\{u_{t}\right\}$ is generated by (2.1), where

(1) $\left\{\epsilon_{t}\right\}$ is i.i.d. with $\mathbb{E}\left(\epsilon_{t}\right)=0, \mathbb{E}\left(\epsilon_{t}^{2}\right)=\sigma_{\varepsilon}^{2}=\left(\sum_{j=0}^{\infty} \psi_{j}^{2}\right)^{-1}$, and $\mathbb{E}\left(\epsilon_{t}^{4}\right)<\infty$.

(2) $\sum_{j=0}^{\infty} j\left|\psi_{j}\right|<\infty$ and the lag polynomial $\Psi(z)=\sum_{j=0}^{\infty} \psi_{j} z^{j} \neq 0$ for all $z \in \mathbb{C}$ and $|z| \leq 1$.

Assumption 2.4. For all $t=1, \ldots, n, D_{t}$ satisfies the conditions:

(i) Let $\mathbb{E}_{t}(\cdot)=\mathbb{E}\left(\cdot \mid \mathcal{F}_{t}\right)$ where $\mathcal{F}_{t}=\sigma\left(\ldots,\left(y_{t-1}, D_{t-1}\right)^{\prime},\left(y_{t}, D_{t}\right)^{\prime}\right)$. For all $s \leq t$ and $i \geq 0, \mathbb{E}\left[\mathbb{E}_{t-i} D_{s} D_{t}-\mathbb{E} D_{s} D_{t}\right]^{2} \leq \zeta_{i}^{2}$, where $\sum_{i=0}^{\infty} i \zeta_{i}<\infty$.

(ii) $\mathbb{E} D_{t}=\mathbb{P}\left(D_{t}=1\right)=p(t / n)$, where $p:[0,1] \rightarrow[\epsilon, 1]$, for some $\epsilon>0$, is a twice continuously differentiable function on $(0,1)$ with $\sup _{0<\tau<1}\left|p^{(i)}(\tau)\right|<$ $\infty$ for $i=1,2$.

(iii) $\operatorname{Cov}\left(D_{t}, D_{t+i}\right)=R_{D, i}\left(\frac{t}{n}, \frac{t+i}{n}\right)$, where each function $R_{D, i}:[0,1]^{2} \rightarrow \mathbb{R}$, $i \geq 0$, is Lipschitz continuous.

(iv) For all $s_{1}, s_{2} \in\{1, \ldots, n\}, \mathbb{E}\left(u_{s_{1}} \mid D_{t}\right)=0$ and $\mathbb{E}\left(u_{s_{1}} u_{s_{2}} \mid D_{t}\right)=\mathbb{E} u_{s_{1}} u_{s_{2}}$.

Assumption 2.1 postulates that the trend $m(\cdot)$ is sufficiently smooth, which is the fundamental assumption for the estimation method to work. While it rules out abrupt structural breaks, this does not appear to be particularly restrictive for climatological applications, as many climatological processes tend to be such that change occurs gradually. In particular, as many series are measured daily or even multiple times a day, only instantaneous breaks, which are extremely unlikely in atmospheric processes, would not be covered by the smooth trend model.

Assumption 2.2 allows for a wide array of unconditional heteroskedasticity. While excluding abrupt breaks, these can be allowed for by generalizing the function $\sigma(\cdot)$ to be piecewise Lipschitz as in Smeekes and Urbain (2014). However, given the limited relevance of abrupt breaks for our climatological focus, we do not pursue this in this thesis.

Assumption 2.3 is a standard linear process assumption that ensures that sufficient moments of $\left\{u_{t}\right\}$ exist and $\left\{u_{t}\right\}$ is weakly dependent and strictly stationary. These assumptions are satisfied by a large class of processes including, but not limited to, all finite order stationary ARMA models. The assumption also implies that $\Omega_{U}=\sigma_{\varepsilon}^{2} \sum_{i=-\infty}^{\infty} \sum_{j=0}^{\infty} \psi_{j} \psi_{j+|i|}<\infty$ (cf. Lemma 2.1). While our current assumption does not allow for conditional heteroskedasticity, this could be relaxed at the expense of increasing the complexity of the theoretical arguments, by allowing $\epsilon_{t}$ 
to be a martingale difference sequence. Similarly, alternative dependence concepts such as mixing, which is considered in the same bootstrap context by Smeekes and Urbain (2014), could be used as well. However, as conditional heteroskedasticity is not the focus of this thesis, we do not consider these extensions.

Assumption 2.4 allows the missing data generating mechanism to be weakly dependent and non-stationary. The mixingale assumption (i) along with the summability condition on $\zeta_{i}$ assures weak dependence and summable autocovariances (Lemma 2.1). For technical reasons, we need to put the mixingale assumption on the product $D_{s} D_{t}$, but it directly implies that $D_{t}$ is a mixingale, as well, by setting $s=t$. By (ii) and (iii), the first two moments of the missing data process are allowed to vary smoothly over time, which thereby allows for instance for smooth periodical changing probabilities (e.g. due to seasonal variation), or long-term changes related to climate change. Smoothness is required as our estimator performs an implicit nonparametric estimate of the missing probability, and thus must behave similarly smooth as the trend function. Assumptions (i)-(iii) are met by a large class of generating processes, including many Markov chains with smoothly varying transition probabilities.

Assumption (iv) can be interpreted as an exogeneity assumption on the missing data generating mechanism, which for instance is satisfied if $\left\{D_{t}\right\}$ is independent of $\left\{u_{t}\right\}$. While this assumption could be argued to be restrictive, it does not appear to be problematic for our focus. Though inconclusive, some recent research has found evidence of a relation between greenhouse gases and the occurrence of cloud cover through climate change, see e.g. Norris et al. (2016). As cloud cover may cause missing observations, our assumption might appear restrictive, but this kind of long-run dependence can be accommodated through the slowly varying trend affecting both $\left\{y_{t}\right\}$ and $\left\{D_{t}\right\}$. As such, the exogeneity assumption mostly rules out short-run effects of ethane on cloud cover and vice versa, which we argue is reasonable.

\subsection{Inference on Trends}

Our goal is to conduct inference on the trend function $m(\cdot)$ defined in Section 2.2. We first describe point estimation of $m(\cdot)$, followed by our bootstrap method, and finally treat the construction of the confidence bands. 


\subsubsection{Estimation of the Trend Function}

We consider local polynomial estimation which is common in the nonparametric regression literature. In particular, we focus on the local constant or NadarayaWatson estimator (Nadaraya, 1964; Watson, 1964), defined as

$$
\begin{aligned}
\hat{m}(\tau) & =\underset{m(\tau)}{\arg \min } \sum_{t=1}^{n} K\left(\frac{t / n-\tau}{h}\right) D_{t}\left\{y_{t}-m(\tau)\right\}^{2} \\
& =\left[\sum_{t=1}^{n} K\left(\frac{t / n-\tau}{h}\right) D_{t}\right]^{-1} \sum_{t=1}^{n} K\left(\frac{t / n-\tau}{h}\right) D_{t} y_{t}, \quad \tau \in(0,1),
\end{aligned}
$$

where $K(\cdot)$ is a kernel function and $h>0$ is a bandwidth, which should satisfy Assumptions 2.5 and 2.6 given below. Note that by construction of the $\left\{D_{t}\right\}$ series, the formulation in (2.2) implies that the estimator only depends on the actually observed data.

Assumption 2.5. $K(\cdot)$ is a symmetric, Lipschitz continuous function with compact support, where we define $\kappa_{k}=\int_{\mathbb{R}} K(\omega)^{k} \mathrm{~d} \omega, \kappa(\tau)=\int_{\mathbb{R}} K(\omega) K(\omega-\tau) \mathrm{d} \omega$ and $\mu_{k}=\int_{\mathbb{R}} \omega^{k} K(\omega) \mathrm{d} \omega$.

Assumption 2.6. The bandwidth $h=h(n)$ satisfies $n h^{7} \rightarrow 0$ and $n h^{2} \rightarrow \infty$ as $n \rightarrow \infty$.

Assumption 2.5 is a standard assumption in the nonparametric kernel smoother literature, and is satisfied by many commonly used kernels. Assumption 2.6 provides the range in convergence rates allowed for $h$ to ensure consistency and asymptotic normality of the kernel estimator.

The bandwidth, or smoothing parameter $h$ plays an important role. Large bandwidths produce a very smooth estimate, while small bandwidths produce a rough, wiggly trend estimate. Although Assumption 2.6 gives some guidance, it does not provide us with a practical bandwidth choice. Data-driven bandwidth selection is therefore important for implementation. Leave-one-out cross-validation is the most popular data-based method for bandwidth selection, but it is designed for independent observations and therefore inappropriate for time series data. Chu and Marron (1991) show that in the presence of positive correlation, this criterion systematically selects very small bandwidths, producing estimates which are too wiggly. With negative correlation, bandwidths will be large and the estimate too smooth. Therefore, Chu and Marron (1991) propose to use a time series version of this criterion, called modified cross-validation (MCV). It is based on minimizing 
the criterion function $\frac{1}{n} \sum_{t=1}^{n} D_{t}\left(\hat{m}_{k, h}\left(\frac{t}{n}\right)-y_{t}\right)^{2}$ with respect to $h$, where

$$
\hat{m}_{k, h}(\tau)=\frac{(n-2 k-1)^{-1} \sum_{t:|t-\tau n|>k} K\left(\frac{t / n-\tau}{h}\right) D_{t} y_{t}}{(n-2 k-1)^{-1} \sum_{t:|t-\tau n|>k} K\left(\frac{t / n-\tau}{h}\right) D_{t}}
$$

is a leave- $(2 k+1)$-out version of the leave-one-out estimator of ordinary crossvalidation, which leaves out the observation receiving the highest weight. Next to formal selection methods, visual inspection of the estimated trend function for a range of different bandwidths can help determining an appropriate bandwidth.

Remark 2.1. The assumption that $p(\cdot)$ is bounded away from zero implies that in every subinterval of $(0,1)$, we have enough observed data points as $n$ grows large. This assumption can be relaxed at the expense of more involved notation by restricting our attention to those compact subsets of $(0,1)$, where the probability of observing data is larger than or equal to some $\epsilon>0$.

In small samples, we may have points $\tau$ around which no data are observed in an $h$-neighborhood. As this is merely a small sample issue, for the theoretical analysis we implicitly assume that $n$ is large enough such that, for every $\tau \in$ $(0,1), \sum_{t=1}^{n} K\left(\frac{t / n-\tau}{h}\right) D_{t} \geq \epsilon^{*}$ for some $\epsilon^{*}>0$. That is, sufficient data are available around $\tau$. This is not restrictive, as by Assumption 2.6, $h$ decreases more slowly than $n$ increases. In practice, the points around which insufficient data are available simply have to be excluded from the set of $\tau$ values considered for inference.

Remark 2.2. While the Nadaraya-Watson estimator locally approximates the trend function by a constant function, the local linear estimator locally fits a linear function to the data around a given point $\tau \in(0,1)$ :

$$
\begin{aligned}
\left(\begin{array}{c}
\hat{m}_{l l}(\tau) \\
\hat{m}_{l l}^{(1)}(\tau)
\end{array}\right) & =\underset{\left(m(\tau), m^{(1)}(\tau)\right)}{\arg \min } \sum_{t=1}^{n} K\left(\frac{t / n-\tau}{h}\right) D_{t}\left\{y_{t}-m(\tau)-m^{(1)}(\tau)(t / n-\tau)\right\}^{2} \\
& =\left(\sum_{t=1}^{n} K\left(\frac{t / n-\tau}{h}\right) \mathbf{x}_{t}(\tau) \mathbf{x}_{t}(\tau)^{\prime} D_{t}\right)^{-1} \sum_{t=1}^{n} K\left(\frac{t / n-\tau}{h}\right) \mathbf{x}_{t}(\tau) D_{t} y_{t}
\end{aligned}
$$

where $\mathbf{x}_{t}(\tau) \equiv(1, t / n-\tau)^{\prime}$. The local linear estimator is more accurate than the local constant estimator at points which are close to the boundaries of the sample. At these points, the local constant estimator suffers from boundary effects which 
the local linear estimator does not (Cai, 2007; Fan, 1992). Our analysis can be extended to the local linear estimator. However, notation becomes significantly more cumbersome and therefore proofs more complicated, so we focus on the local constant estimator in the theoretical part.

Remark 2.3. An alternative way to specify the estimators in the presence of missing data is to work with unequally spaced data. Assuming we observe data on times $t_{i}$ for $i=1, \ldots, n_{1}$, where $n_{1}$ is the effective number of observations, the local constant estimator can be written as

$$
\begin{aligned}
\hat{m}(\tau)= & {\left[\sum_{i=1}^{n_{1}} K\left(\frac{\left(t_{i}-t_{1}\right) /\left(t_{n_{1}}-t_{1}\right)-\tau}{h}\right)\right]^{-1} } \\
& \times \sum_{i=1}^{n_{1}} K\left(\frac{\left(t_{i}-t_{1}\right) /\left(t_{n_{1}}-t_{1}\right)-\tau}{h}\right) y_{t_{i}} .
\end{aligned}
$$

This formulation has the advantage that it does not require an underlying regular frequency at which the data are observed. However, in many applications it is not hard to define such an underlying frequency (days in our application), and then the two formulations are equivalent.

In the remainder of the chapter we will continue to work with the first formulation, which leads to clearer notation and is easier to handle in the proofs, as the randomness in the missing data is modeled through the explicit $D_{t}$ variable rather than being "hidden" in the (now random) $t_{i}$ dates.

\subsubsection{Autoregressive Wild Bootstrap}

To construct confidence bands around the trend estimate, we modify the wild bootstrap, originally designed to handle heteroskedastic data (Davidson and Flachaire, 2008), to account for serial dependence. The wild bootstrap generates bootstrap errors as $z_{t}^{*}=\xi_{t}^{*} \hat{z}_{t}$, where $\hat{z}_{t}$ are residuals of the nonparametric trend regression. In the standard wild bootstrap, the random variables $\left\{\xi_{t}^{*}\right\}$ are i.i.d. and thus, any dependence present in the data gets removed in the bootstrap errors. To overcome this drawback, Shao (2010b) proposed the dependent wild bootstrap (DWB) in which $\left\{\xi_{t}^{*}\right\}$ are generated as $\ell$-dependent random variables with $\operatorname{Cov}\left(\xi_{s}^{*}, \xi_{t}^{*}\right)=K_{D W B}\left(\frac{s-t}{\ell}\right)$, where $K_{D W B}(\cdot)$ is a kernel function. As the tuning parameter $\ell$, it has to be selected by the user.

Building on this idea, Smeekes and Urbain (2014) propose the autoregressive wild bootstrap (AWB) where $\left\{\xi_{t}^{*}\right\}$ is generated as an $\mathrm{AR}(1)$ process with parameter 
$\gamma=\gamma(n)$. The AWB has as advantage over the DWB that it is easier to implement and has a more intuitive interpretation. Moreover, as $\left\{\xi_{t}^{*}\right\}$ is not $\ell$-dependent, the AWB has the potential to capture more serial correlation and to be less sensitive to the choice of tuning parameter $\gamma$. In the context of unit root testing, Smeekes and Urbain (2014) show that the AWB generally has a superior finite sample performance compared to the DWB. For these reasons, we mainly focus on the AWB in the following, although we consider the DWB in our simulation study as well. The AWB algorithm can be described as follows.

Algorithm 2.1 (Autoregressive Wild Bootstrap).

1. Let $\tilde{m}(\cdot)$ be defined as in $(2.2)$, but using bandwidth $\tilde{h}$. Obtain residuals $\hat{z}_{t}=D_{t}\left[y_{t}-\tilde{m}(t / n)\right]$ for $t=1, \ldots, n$.

2. For $0<\gamma<1$, generate $\nu_{1}^{*}, \ldots, \nu_{n}^{*}$ as i.i.d. $\mathcal{N}\left(0,1-\gamma^{2}\right)$ and let $\xi_{t}^{*}=\gamma \xi_{t-1}^{*}+\nu_{t}^{*}$ for $t=2, \ldots, n$. Take $\xi_{1}^{*} \sim \mathcal{N}(0,1)$ to ensure stationarity of $\left\{\xi_{t}^{*}\right\}$.

3. Calculate the bootstrap errors $z_{t}^{*}$ as $z_{t}^{*}=D_{t} \xi_{t}^{*} \hat{z}_{t}$ and generate the bootstrap observations by $y_{t}^{*}=D_{t}\left[\tilde{m}(t / n)+z_{t}^{*}\right]$ for $t=1, \ldots, n$, where $\tilde{m}(\cdot)$ is the same estimate as in the first step.

4. Obtain the bootstrap estimator $\hat{m}^{*}(\cdot)$ as defined in (2.2) using the bootstrap series $\left\{y_{t}^{*}\right\}$, with the same bandwidth $h$ as used for the original estimate $\hat{m}(\cdot)$.

5. Repeat Steps 2 to $4 B$ times, and let

$$
\hat{q}_{\alpha}(\tau)=\inf \left\{u \in \mathbb{R}: \mathbb{P}^{*}\left[\hat{m}^{*}(\tau)-\tilde{m}(\tau) \leq u\right] \geq \alpha\right\}
$$

denote the $\alpha$-quantile of the $B$ centered bootstrap statistics $\hat{m}^{*}(\tau)-\tilde{m}(\tau)$. These bootstrap quantiles are then used to construct confidence bands as described below.

Note that in Step 3 we only have to draw bootstrap observation for the dates where $D_{t}=1$ and we observed the realization $y_{t}$; in the description the missing ones are artificially set to zero, but they are actually not used anywhere. In Step 2, we generate $\left\{\xi_{t}^{*}\right\}$ for all $t=1, \ldots, n$, although subsequently we only use the subset that corresponds to the actually observed data points. The missing data structure is preserved in the bootstrap sample, while the correlation between consecutive non-missing observations is determined only by their distance, which ensures a 
coherent bootstrap sample. In this way, the missing data structure is automatically taken into account in the bootstrap without any need for modifications.

Although we suggest to generate $\left\{\nu_{t}^{*}\right\}$ as a sequence of normally distributed random variables, inspection of the proofs shows normality is not needed; all one needs is a sequence of i.i.d. random variables with $\mathbb{E}^{*} \nu_{t}^{*}=0, \mathbb{E}^{*} \nu_{t}^{* 2}=1-\gamma^{2}$ and $\mathbb{E}^{* 4} \nu_{t}^{* 4}<\infty$. Normality is simply a convenient option that is easy to implement; alternatively one could implement a variant of the Rademacher distribution (corrected for the right variance), which for the independent wild bootstrap has good properties (Davidson and Flachaire, 2008).

For the tuning parameter $\gamma$, we follow Smeekes and Urbain (2014) and let $\gamma=\theta^{1 / \ell}$ where $\ell$ is the "block length" parameter also found in the DWB and $0<\theta<1$ is a fixed parameter. This specification has the advantage that $\ell$ can be interpreted in a similar way as the block length parameter in a block bootstrap; its choice constitutes a trade-off between capturing more of the dependence with a large value of the tuning parameter, and allowing for more variation in the bootstrap samples with a smaller value for $\ell$. Additionally, it provides a convenient framework for studying the theoretical properties of our method. Specifically, we need $\ell \rightarrow \infty$ as $n \rightarrow \infty$, such that $\gamma \rightarrow 1$. This is analogous to the block (and dependent wild) bootstrap, where the block size must increase to capture more dependence when the sample size increases. Assumption 2.7 postulates the formal conditions that $\ell$ needs to satisfy. They imply that $\gamma \rightarrow 1$, but not too fast.

Assumption 2.7. The bootstrap parameter $\ell=\ell(n)$ satisfies $\ell \rightarrow \infty$ and $\ell / \sqrt{n h} \rightarrow 0$ as $n \rightarrow \infty$.

Note that we propose to use a different bandwidth $\tilde{h}$ in Step 1 of the algorithm. This is a common feature in the literature on bootstrap methods for nonparametric regression. By either selecting a larger (oversmoothing) or smaller bandwidth (undersmoothing) than used for the estimator, one can account for the asymptotic bias that is present in the local polynomial estimation, see Hall and Horowitz (2013, Section 1.4) for an extensive literature review. While undersmoothing, such as used in the related paper by Neumann and Polzehl (1998), aims at making the bias asymptotically negligible, oversmoothing aims at producing a consistent estimator of the (non-negligible) bias. Both have advantages and disadvantages, see the extensive discussion in Hall and Horowitz (2013). We follow Bühlmann (1998) and consider a solution based on oversmoothing, which we find to work well in practice; also see Hardle and Marron (1991). After presenting our theoretical 
results in Section 2.4, Remark 2.8 provides an intuition of why oversmoothing allows to consistently estimate the asymptotic bias. ${ }^{2}$ We now state the formal conditions that $\tilde{h}$ must satisfy in Assumption 2.8 ; one is that $h / \tilde{h} \rightarrow 0$ as $n \rightarrow \infty$, which ensures the oversmoothing.

Assumption 2.8. The oversmoothing bandwidth $\tilde{h}=\tilde{h}(n)$ satisfies $\max \left\{\tilde{h}, h / \tilde{h}, n h^{5} \tilde{h}^{4}\right\} \rightarrow 0$ and $\ell \max \left\{\tilde{h}^{4}, 1 / n \tilde{h}\right\} \rightarrow 0$ as $n \rightarrow \infty$.

Remark 2.4. There are a number of ways to choose the AWB parameter $\gamma$ in practice. Using the relation $\gamma=\theta^{1 / \ell}$, one can fix $\theta$ and choose $\ell$ as a deterministic function of the sample size. Smeekes and Urbain (2014) found that $\theta=0.01$ paired with $\ell=1.75 n^{1 / 3}$ performed well in their simulation study on AWB unit root testing; for the local polynomial estimation we might adapt this to let $\ell$ be a function of $n h$, see also Remark 2.5. Alternatively, one may vary $\gamma$ over a range of reasonable values, choosing a value in the range where the bands are most stable (as a function of $\gamma$ ), akin to the minimum volatility method proposed by Politis et al. (1999). Ideally, one would like to have a data-driven method for choosing an "optimal" $\gamma$. However, development of such a method requires a deeper study of higher-order asymptotic properties, which is outside the scope of this thesis.

Remark 2.5. To give some intuition for the interaction between the three tuning parameters $h, \tilde{h}$ and $\ell$, consider taking $h=c n^{-p}$, for some $c>0$ and $\frac{1}{7}<p<\frac{1}{2}$. This satisfies Assumption 2.6. Now let $\tilde{h}=c n^{-q}$, where $p>q$ as $h / \tilde{h} \rightarrow 0$. Furthermore, to satisfy $n h^{5} \tilde{h}^{4} \rightarrow 0$, we need that $5 p+4 q>1$. For $p \geq \frac{1}{5}$ this is satisfied for any $q$; with $p$ approaching $\frac{1}{7}, q>\frac{1}{14}$ suffices, which is not very strict.

Finally, take $\ell=c n^{r}$. From Assumption 2.7 we know that $r<\frac{1}{2}-p / 2$, which for $p$ approaching $\frac{1}{2}$ means $r<\frac{1}{4}$, while for $p$ approaching $\frac{1}{7}$, it implies that $r<\frac{3}{7}$. To satisfy the restrictions from Assumption 2.8, we need that $r<4 q$ and $r<1-q$. As $q<p<\frac{1}{2}$, the second condition is non-binding. The first condition is only restrictive when $q$ is small, say in the vicinity of $\frac{1}{14}$. Then $r<\frac{2}{7}$, which is stricter than the condition implied by Assumption 2.7. However, for $q \geq \frac{3}{28}$, this restriction is not binding.

As an example, consider the "classical" rates $p=\frac{1}{5}$ and $q=\frac{1}{9}$, cf. Bühlmann (1998). These are allowed under our theory, and additionally imply that $0<r<\frac{2}{5}$, therefore also allowing for $r=\frac{1}{3}$ as advocated in Smeekes and Urbain (2014).

\footnotetext{
${ }^{2}$ Hall and Horowitz (2013) propose an alternative bootstrap approach that requires neither under- nor oversmoothing, however their approach only delivers pointwise intervals, and is therefore not considered in this chapter.
} 
Remark 2.6. Instead of the autoregressive wild bootstrap, one could equally imagine a moving-average wild bootstrap (MA-WB), where $\xi_{t}^{*}=\sum_{j=0}^{\ell} \psi_{n, j} \nu_{t}^{*}$ and $\nu_{t}^{*}$ are i.i.d. random variables as before. By letting $\psi_{n, j} \rightarrow 1$, if $n \rightarrow \infty$ and $j$ fixed, and $\psi_{n, j} \rightarrow 0$, if $n$ is fixed and $j \rightarrow \infty$, one could show validity of such an MA-WB as well. For practical purposes, one could take $\psi_{n, j}=f(j / \ell)$, for instance with $f(x)=1-x^{r}$ to simplify implementation.

Such a moving-average representation is closely related to the DWB, which can be seen as a two-sided MA process, with both bootstrap methods delivering $\ell$-dependent bootstrap samples. In his paper, Shao (2010b) shows the asymptotic equivalence of the variance estimator of the DWB with the tapered block bootstrap (Paparoditis and Politis, 2002). Similarly, one can show that the MA-WB has a close link to the extended tapered wild bootstrap of Shao (2010a), and depending on the distribution chosen for $\left\{\nu_{t}\right\}$, the smooth extended tapered block bootstrap of Gregory et al. $(2015,2018)$. If $\left\{\nu_{t}\right\}$ is drawn from a continuous distribution like the normal, we have an automatic smoothing in the wild bootstrap variants. As Gregory et al. $(2015,2018)$ show that smoothing helps in the context of the block bootstrap, it may similarly do so for the wild bootstrap and provide a potential reason to prefer the normal distribution over discrete distributions like the Rademacher distribution.

\subsubsection{Bootstrap Confidence Bands}

Pointwise bootstrap confidence intervals with a confidence level of $(1-\alpha)$ for $m(\tau)$, are denoted by $I_{n, \alpha}^{(p)}(\tau)$ and constructed with the objective that

$$
\liminf _{n \rightarrow \infty} \mathbb{P}\left[\left(m(\tau) \in I_{n, \alpha}^{(p)}(\tau)\right)\right] \geq 1-\alpha \quad \tau \in(0,1) .
$$

Using our bootstrap algorithm, we can construct such pointwise intervals as

$$
I_{n, \alpha}^{(p)}(\tau)=\left[\hat{m}(\tau)-\hat{q}_{1-\alpha / 2}(\tau), \hat{m}(\tau)-\hat{q}_{\alpha / 2}(\tau)\right]
$$

As these intervals are constructed separately for each $\tau$, links over time cannot be established with these intervals. Therefore, we next consider how to construct simultaneous confidence bands. Let $I_{n, \alpha}^{G}(\tau)$, for $\tau \in G$, denote a confidence band that is simultaneous over the set $G$. Formally, we seek to construct $I_{n, \alpha}^{G}(\tau)$ such 
that

$$
\liminf _{n \rightarrow \infty}\left[\mathbb{P}\left(m(\tau) \in I_{n, \alpha}^{G}(\tau) \quad \forall \tau \in G\right)\right] \geq 1-\alpha
$$

Our practical implementation follows the three-step procedure proposed by Bühlmann (1998):

1. For all $\tau \in G$, obtain pointwise quantiles $\hat{q}_{\alpha_{p} / 2}(\tau), \hat{q}_{1-\alpha_{p} / 2}(\tau)$ for varying $\alpha_{p} \in[1 / B, \alpha]$.

2. Choose $\alpha_{s}$ such that

$$
\alpha_{s}=\underset{\alpha_{p} \in[1 / B, \alpha]}{\arg \min }\left|\mathbb{P}^{*}\left[\hat{q}_{\alpha_{p} / 2}(\tau) \leq \hat{m}^{*}(\tau)-\tilde{m}(\tau) \leq \hat{q}_{1-\alpha_{p} / 2}(\tau) \forall \tau \in G\right]-(1-\alpha)\right|
$$

3. Construct the simultaneous confidence bands as

$$
I_{n, \alpha}^{G}(\tau)=\left[\hat{m}(\tau)-\hat{q}_{1-\alpha_{s} / 2}(\tau), \hat{m}(\tau)-\hat{q}_{\alpha_{s} / 2}(\tau)\right] \quad \tau \in G
$$

In the second step, a pointwise error $\alpha_{s}$ is found for which a fraction of approximately $(1-\alpha)$ of all centered bootstrap estimates falls within the resulting confidence intervals, for all points of the set $G$. As such, the confidence intervals with pointwise coverage $\left(1-\alpha_{s}\right)$ become simultaneous confidence bands with coverage $(1-\alpha)$.

Remark 2.7. As an alternative to the variable-size bands proposed by Bühlmann (1998), consider Kolmogorov-Smirnov-type simultaneous confidence bands of fixed size. They would be of the form

$$
I_{\alpha}^{*}(\tau)=\left[\hat{m}(\tau)-t_{1-\alpha}^{*}, \hat{m}(\tau)+t_{1-\alpha}^{*}\right]
$$

where the quantile $t_{1-\alpha}^{*}$ is determined as the $(1-\alpha)$-quantile of the distribution of the quantity $U_{n}^{*}=\sup _{\tau \in G}\left\{\left|\hat{m}^{*}(\tau)-\tilde{m}(\tau)\right|\right\}$. Neumann and Polzehl (1998) establish the asymptotic validity of such simultaneous bands under serial independence. We do not go in this direction, because we believe confidence bands with variable width to be more informative. They have the feature of becoming wider at points with more variability and more narrow for periods with less variability. To obtain variable width intervals with the Kolmogorov-Smirnov approach, one has to estimate the variance of the estimator at each $\tau$ and bootstrap a pivotal quantity, see 
e.g. Neumann and Polzehl (1998, Section 2.2). This adds additional complications in order to achieve consistent variance estimation.

\subsection{Asymptotic Theory}

We first provide the pointwise limiting normal distribution of the local constant estimator $\hat{m}(\cdot)$. Although the result is similar to the non-bootstrap part of Theorem 3.1 in Bühlmann (1998), we extend the asymptotic theory for the local constant estimator to allow for the presence of nonstationary volatility and missing data. As we feel this is a noteworthy result in its own right, we present it in Theorem 2.1 .

Theorem 2.1. Under Assumptions 2.1-2.6, for any $\tau \in(0,1)$, we have as $n \rightarrow \infty$ :

$$
\sqrt{n h}\left(\hat{m}(\tau)-m(\tau)-h^{2} B_{a s}(\tau)\right) \stackrel{d}{\rightarrow} \mathcal{N}\left(0, \sigma_{a s}^{2}(\tau)\right),
$$

where

$$
B_{a s}(\tau)=\mu_{2} p(\tau)^{-1}[m p]^{(2)}(\tau) \quad \text { and } \quad \sigma_{a s}^{2}(\tau)=p(\tau)^{-1} \sigma(\tau)^{2} \Omega_{U} \kappa_{2},
$$

The term $B_{a s}(\tau)$ reflects the familiar asymptotic bias generally found in local polynomial estimators, although the exact form is different due the presence of the missing data parameter $p(\tau)$. The asymptotic variance $\sigma_{a s}^{2}(\tau)$ is not only affected by $p(\tau)$, but also by the volatility process $\sigma^{2}(\tau)$. If one were to use these distributions directly for inference, one would need to plug in consistent estimators of these nuisance parameters. However, as we show next, in the bootstrap these are automatically consistently estimated, and we have consistency of the autoregressive wild bootstrap method for the local constant estimator.

Theorem 2.2. Under Assumptions 2.1-2.8, for any $\tau \in(0,1)$, we have as $n \rightarrow \infty$ :

$$
\sqrt{n h}\left(\hat{m}^{*}(\tau)-\hat{m}(\tau)-h^{2} B_{a s}(\tau)\right){\stackrel{d^{*}}{\rightarrow}}_{p} \mathcal{N}\left(0, \sigma_{a s}^{2}(\tau)\right),
$$

where $B_{a s}(\tau)$ and $\sigma_{a s}^{2}(\tau)$ are defined in $(2.8)$.

The pointwise validity of the bootstrap confidence intervals in the sense of (2.6) follows directly from this pointwise convergence result. Note that, as the bias term $B_{a s}(\tau)$ is the same in both theorems, it is consistently estimated by the bootstrap. As such, we do not need the bias to disappear, which happens when 
undersmoothing if $n h^{5} \rightarrow 0$, or to be $O(1)$, when $n h^{5} \rightarrow c$. Even if $n h \rightarrow \infty$, and the asymptotic bias dominates the stochastic variation, the bootstrap correctly mimics this and can be used for asymptotically valid inference. As such, we can relax the assumption in Bühlmann (1998, p. 55) that $h \sim C n^{-1 / 5}$ to allow for a wider range of bandwidths. In practice, this means that the bootstrap provides additional protection against a misspecified bandwidth, by letting the widths of confidence bands automatically adapt.

Next, to study the validity of simultaneous confidence bands as in (2.7), we consider $h$-neighborhoods around time points $\tau$. We do so because estimates $\hat{m}\left(\tau_{1}\right)$ and $\hat{m}\left(\tau_{2}\right)$ are asymptotically independent for $\tau_{1} \neq \tau_{2}$ being two fixed distinct time points. When the distance between $\tau_{1}$ and $\tau_{2}$ is of order $h$, the estimators show a non-zero correlation. Therefore a major benefit of "zooming in" on local $h$-neighborhoods is that we can study how the bootstrap mimics the correlation between close points, a feature which is lost when considering simultaneity globally.

Theorem 2.3. For any $\tau_{0} \in(0,1)$, let

$$
\begin{aligned}
& Z_{\tau_{0}, n}(\tau)=\sqrt{n h}\left(\hat{m}\left(\tau_{0}+\tau h\right)-m\left(\tau_{0}+\tau h\right)\right), \\
& Z_{\tau_{0}, n}^{*}(\tau)=\sqrt{n h}\left(\hat{m}^{*}\left(\tau_{0}+\tau h\right)-\tilde{m}\left(\tau_{0}+\tau h\right)\right) .
\end{aligned}
$$

Then, under Assumptions 2.1-2.8, we have for all $\tau_{0} \in(0,1)$

$$
\begin{aligned}
& \left\{Z_{\tau_{0}, n}(\tau)-B_{a s}\left(\tau_{0}\right)\right\}_{\tau \in[-1,1]} \Rightarrow\{W(\tau)\}_{\tau \in[-1,1]}, \\
& \left\{Z_{\tau_{0}, n}^{*}(\tau)-B_{a s}\left(\tau_{0}\right)\right\}_{\tau \in[-1,1]} \Rightarrow_{p}\{W(\tau)\}_{\tau \in[-1,1]},
\end{aligned}
$$

where $\{W(\tau)\}_{\tau \in[-1,1]}$ is a Gaussian process with $\mathbb{E} W(\tau)=0$ and

$$
\operatorname{Cov}\left(W\left(\tau_{1}\right), W\left(\tau_{2}\right)\right)=\sigma_{W, \tau_{0}}\left(\tau_{1}, \tau_{2}\right)=p\left(\tau_{0}\right)^{-1} \sigma\left(\tau_{0}\right)^{2} \Omega_{U} \kappa\left(\tau_{1}-\tau_{2}\right)
$$

Here, $\Rightarrow$ denotes weak convergence in the space of continuous real-valued functions on $[-1,1]$ endowed with the sup-norm.

Theorem 2.3 establishes the uniform validity of the bootstrap within an $h$ neighborhood around any point $0<\tau_{0}<1$, where, since $h=o(1)$, we assume without loss of generality that $m\left(\tau_{0}+\tau h\right)$ is always defined. Note that the interval $[-1,1]$ is mainly chosen out of convenience, and the results can trivially be shown to hold over any interval $\left[\tau_{0}-a h, \tau_{0}+b h\right]$ with $0<a, b<\infty$. Moreover, it follows directly from Theorem 2.3 that the bootstrap will be valid uniformly on sets that 
contain a union of any finite number of such $h$-neighborhoods, see e.g. Bühlmann (1998, Corollary 3.3). While, in finite samples, one can always take $h$ and the intervals such that the full sample is covered in $G$, this kind of "too large" simultaneity should be considered with caution, as this is not what the asymptotic analysis covers. Although simultaneity over such local sets might appear less attractive than simultaneity over the whole sample, it can nevertheless be of great interest in applications. For example, constructing confidence bands with simultaneous coverage over two time periods - one located early in the sample and the other one at the end - is useful when judging if there was an upward (or downward) movement of the trend at the end of the time period when compared to the beginning. This allows the empirical researcher to draw conclusions about developments spanning time stretches, which is not possible with pointwise confidence intervals.

Remark 2.8. To provide some intuition for the required oversmoothing with bandwidth $\tilde{h}$ in the bootstrap, note that from Theorem 2.1 (or more formally Lemma 2.6) we can deduce that the estimator used in the first step of the bootstrap algorithm satisfies

$$
\tilde{m}(\tau)-m(\tau)=\tilde{h}^{2} B_{a s}(\tau)+Z_{n}(\tau) / \sqrt{n \tilde{h}}+o_{p}(1 / \sqrt{n \tilde{h}})
$$

where $Z_{n}(\tau) \stackrel{d}{\rightarrow} N\left(0, \sigma_{\text {as }}^{2}(\tau)\right)$. Furthermore, letting

$$
w_{t, n}(\tau)=K\left(\frac{t / n-\tau}{h}\right) D_{t} /\left[\sum_{t=1}^{n} K\left(\frac{t / n-\tau}{h}\right) D_{t}\right]
$$

and using that $y_{t}^{*}=\tilde{m}(t / n)+z_{t}^{*}$, we can write

$$
\hat{m}^{*}(\tau)-\tilde{m}(\tau)=\left[\sum_{t=1}^{n} w_{t, n}(\tau) \tilde{m}(t / n)-\tilde{m}(\tau)\right]+\sum_{t=1}^{n} w_{t, n}(\tau) z_{t}^{*}+o_{p}(1 / \sqrt{n \tilde{h}})
$$

While the second term, $\sum_{t=1}^{n} w_{t, n}(\tau) z_{t}^{*}$, mimics the stochastic variation in the trend estimation, and ensures the asymptotic normality of the bootstrap trend estimator, the bias arises from the first term, $\sum_{t=1}^{n} w_{t, n}(\tau) \tilde{m}(t / n)-\tilde{m}(\tau)$. Using 
(2.9), this term can be decomposed as

$$
\begin{aligned}
\sum_{t=1}^{n} w_{t, n}(\tau) \tilde{m}(t / n)-\tilde{m}(\tau)= & {\left[\sum_{t=1}^{n} w_{t, n}(\tau) m(t / n)-m(\tau)\right] } \\
& +\left[\sum_{t=1}^{n} w_{t, n}(\tau) B_{a s}(t / n)-B_{a s}(\tau)\right] \tilde{h}^{2} \\
& +\left[\sum_{t=1}^{n} w_{t, n}(\tau) Z_{n}(t / n)-Z_{n}(\tau)\right] / \sqrt{n \tilde{h}} \\
& +o_{p}(1 / \sqrt{n \tilde{h}}) .
\end{aligned}
$$

The asymptotic bias arises, as for the original estimator, from the first term of the decomposition. As shown in the proof of Lemma 2.7, by the smoothness of $B_{a s}(\tau)$, the second term converges to zero, thus canceling out the bias in $\tilde{m}(\tau)$. However, to make the third, stochastic term vanish when multiplying by $\sqrt{n h}$, it must be that $h / \tilde{h} \rightarrow 0$, such that this term is of small enough magnitude. This is achieved by oversmoothing.

Remark 2.9. Although it is not visible from the theorems - as we only consider pointwise $\tau$ (or $\tau_{0}$ in Theorem 2.3) - $\tau$ needs to be bounded away from the boundaries ( 0 and 1$)$ to make the results hold. As the estimator exhibits edge effects, the quality of $\tilde{m}(\tau)$ can only be guaranteed for $\tau$ away from 0 or 1 . Formally, we need to take a small $\delta>0$ and then consider $\tau \in[\delta, 1-\delta]$. Consequently, in the bootstrap we then obtain the limit distribution in a slightly smaller set $\tau \in\left[\delta^{*}, 1-\delta^{*}\right]$, for some $\delta^{*}>\delta$. However, as we can take $\delta$ and $\delta^{*}$ as small as we like, this does not affect the pointwise statements of the theorems. ${ }^{3}$

Because of the above reasons, Bühlmann (1998, p. 53) suggests in his bootstrap algorithm to obtain residuals $\hat{z}_{t}=D_{t}\left[y_{t}-\tilde{m}(t / n)\right]$ for $t=[n \delta]+1, \ldots,[n(1-\delta)]$, as the residuals too close to the boundary may contaminate the bootstrap sample when they are sampled. This is not a problem for our method, as the AWB does not involve resampling: boundary residuals remain at the boundary in the bootstrap sample, and can therefore not affect results away from the boundaries.

\footnotetext{
${ }^{3}$ See Lemmas 2.6 and 2.7 in Appendix A for statements where these constants do appear.
} 


\subsection{Simulation Study}

For the simulation exercise, we simulate time series with a trending behavior in both mean and variance, inspired by patterns observed in climatological time series, and allow for similar patterns of missing data. We will first describe the setting and then present and discuss the results.

\subsubsection{Simulation Setup}

We consider the following smooth transition model:

$$
y_{t}=m(t / n)+\sigma_{t} u_{t}, \quad m(\tau)=\beta_{1} \tau+\beta_{2} \tau G(\tau, \lambda, c),
$$

where for $\lambda>0$,

$$
G(\tau, \lambda, c)=(1+\exp \{-\lambda(\tau-c)\})^{-1}
$$

The error term $\left\{u_{t}\right\}$ follows an $\operatorname{ARMA}(1,1)$ model

$$
u_{t}=\phi u_{t-1}+\psi \epsilon_{t-1}+\epsilon_{t} \quad \epsilon_{t} \sim \mathcal{N}\left(0, \frac{\left(1-\phi^{2}\right) / 4}{1+\psi^{2}-2 \phi \psi}\right),
$$

where we vary the parameters $\phi$ and $\psi$ to investigate the impact of serial correlation on our method. The variance of $\epsilon_{t}$ is normalized such that the signal to noise ratio does not depend on the specific choice of the AR and MA parameter. Furthermore, we introduce heteroskedasticity with the process $\left\{\sigma_{t}\right\}$. We consider two scenarios, where $\sigma_{t}$ is constant over time or $\sigma_{t}=\sigma(t / n)$, with the volatility process $\sigma(\tau)$ given by

$$
\sigma(\tau)=\sigma_{0}+\left(\sigma_{*}-\sigma_{0}\right)(\tau)+a \cos (2 \pi k \tau)
$$

Equation (2.10) is a shifting mean model as considered by Gonzalés and Teräsvirta (2008), and can be seen as a smooth transition version of a broken trend model with one break. The function $G(\tau, \lambda, c)$ as given in (2.11) is the transition function with time as transition variable. Its inputs apart from time are the location of the shift - the parameter $c$ - as well as the smoothness of the shift, determined by $\lambda$. For large values of $\lambda$ the shift happens almost instantaneous, while it is smoother for smaller values of this parameter. In our simulations, we fix $\lambda=10$. The other parameters of our DGP will be chosen in such a way that the time series 
experiences a downward trend during the first three quarters which turns into a steeper upward trend in the last quarter.

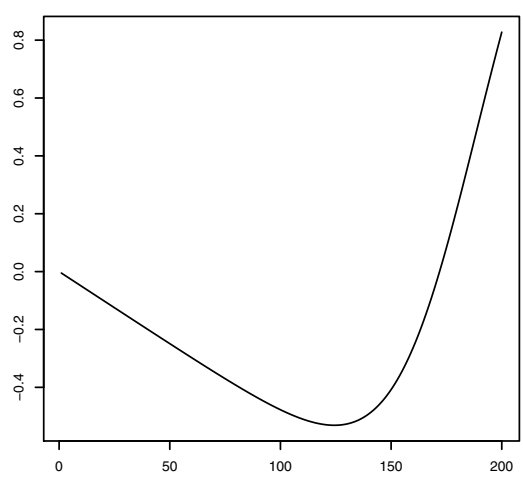

(a) The trend function $m(\tau)$

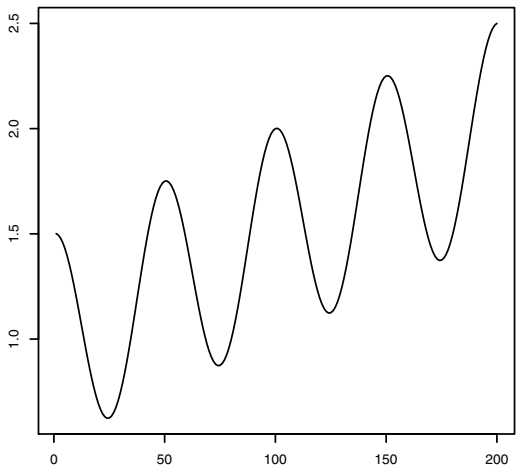

(b) The variance process $\sigma(\tau)$ with $k=4$ and $a=0.5$

Figure 2.1: Trends in mean and variance in the simulation DGP

This mimics the general pattern which is expected to occur in atmospheric ethane time series and therefore fits our application well. More specifically, this means we set the location of the shift to occur at $c=0.9$. The slope of the trend gradually changes from $\beta_{1}=-1$ before the shift to $\beta_{2}=2.5$ after the shift. This is illustrated in Figure 2.1(a). For the variance process, inspired by the series considered in the empirical application, we consider a cyclical component with trend. We have to choose four parameters in (2.13): the start and end point of the trend $-\sigma_{0}$ and $\sigma_{*}-$ as well as the specifics of the cyclical component. The parameter $a$ fixes the amplitude of the cycle, while $k$ determines how many cycles there are. We set $\sigma_{0}=1, \sigma_{*}=2$ and consider different combinations of values for $a$ and $k$. We let $a=0.3,0.5,0.7$ and $k=2,3,4$. An example of this process is displayed in Figure 3.6(b).

We also consider different degrees of dependence by varying the AR and MA parameters. For the AR parameter we take $\phi=0,0.2,0.5,-0.5$, while the MA parameter varies between $\psi=0, \psi=0.2$ and $\psi=0.5$. We only look at pure AR or MA processes with these coefficient values. The different specifications will be abbreviated in the tables with self-explanatory names, e.g. we write $A R_{-0.5}$ for $\phi=-0.5, \psi=0$ and use $M A_{0.5}$ when $\phi=0$ and $\psi=0.5$. 
In addition, we consider cases of missing data for which we generate a missing pattern that is representative for the ethane data. We implement a first-order Markov Chain for $D_{t}$ with transition probabilities

$$
\begin{array}{r}
D_{t}=0 \\
D_{t-1}=0 \\
D_{t-1}=1
\end{array}\left(\begin{array}{cc}
0.80 & 0.20 \\
0.45 & 0.55
\end{array}\right),
$$

which are estimated from the ethane time series considered in Section 2.6. This transition matrix results in an average fraction of around $70 \%$ missing observations.

In the estimation step, we apply the local constant estimator based on the Epanechnikov kernel which is given by the function $K(x)=\frac{3}{4}\left(1-x^{2}\right) \mathbb{1}_{\{|x| \leq 1\}}$. For the bandwidth parameter $h$ we use $h=0.02, h=0.04$ and $h=0.06$. In the first step of the bootstrap procedure we follow the recommendation of Bühlmann (1998) to use $\tilde{h}=C h^{5 / 9}$ with $C=2$. In the second step of the bootstrap, we consider different values for the $\mathrm{AR}$ parameter $\gamma$; next to $\gamma=0$, which reduces the AWB to a standard wild bootstrap (WB), we also consider $\gamma=0.2,0.4,0.6$.

For each specification, we run 5000 Monte Carlo simulations. We report average pointwise as well as simultaneous coverage for a sample size of $n=200$, based on $B=999$ bootstrap replications. For ease of comparison, we choose the sample size in cases with missing data such that we have approximately 200 data points remaining. Given the large fraction of missing data, an expected effective sample size of 200 translates into an original sample size for our Markov Chain of $n=666$.

The nominal coverage in all cases is $95 \%$. We also report the average median length of the confidence intervals in parenthesis underneath the respective coverage. For simultaneous coverage, the trend curve has to lie within the confidence bands for all points of the considered set $G$, for which we take the two sets $G_{\text {sub }}$ and $G$ considered by Bühlmann (1998), where $G_{s u b}=U_{1}(h) \cup U_{4}(h)$ and $G=\bigcup_{i=1}^{4} U_{i}(h)$, with $U_{i}(h)=\{(i / 5)-h+j / 100 ; j=0, \ldots,[200 h]\}$.

To compare the performance of our AWB method to related bootstrap methods, we also implement the dependent wild bootstrap (DWB) and the sieve wild bootstrap (SWB), with a standard normal distribution for generation of the wild bootstrap errors. Since the SWB cannot easily be adapted to work with missing data, we provide results for this method only for the cases with no missing data. For the DWB, we convert the $\gamma$ parameter into the corresponding value for the tuning parameter $\ell$, using the formula $\gamma=\theta^{1 / \ell}$. Smeekes and Urbain (2014) found in their simulation study that $\theta=0.01$ provides a sensible conversion be- 
tween the AWB and DWB, in the sense of yielding comparable performance of the two methods, therefore we use $\theta=0.01$ as well to convert the AWB parameter into the DWB parameter. This tuning parameter does not exist in the case of the SWB; instead, the lag length has to be selected, for which we use AIC.

\subsubsection{Simulation Results}

First, we report results for equally spaced data with no missing observations and a variance process with the same specifications $(k=4$ and $a=0.5)$ as displayed in Figure 3.6(b). The results on pointwise coverage are given in Table 2.1, while Tables 2.3 and 2.2 show simultaneous coverage probabilities for the two sets G.sub and $G$, respectively. The tables consist of three main blocks, one for each bandwidth. Within each block, the individual rows contain results for different combinations of AR and MA parameters. Results for other choices of the variance parameters $k$ and $a$, including the homoskedastic case, are available in Supplementary Appendix C. Qualitatively, these settings yield the same conclusions as the ones considered here.

Table 2.1 shows that the autoregressive wild bootstrap provides confidence intervals with good pointwise coverage in the presence of heteroskedasticity and mild autocorrelation. For the independent case and the cases with negative or small positive correlation, the coverage probabilities are close to the nominal level. The only specifications for which the coverage lies below the nominal level are when $\phi=0.5$ and $\psi=0.5$. In these cases, the data deviate from the trend line in clusters due to the strong positive correlation. This causes the nonparametric estimate to go through these clusters and thus, to deviate significantly from the true trend. The confidence bands are in these situations not wide enough to cover the true trend, resulting in too low coverage. Interestingly, all methods/tuning parameters are similarly affected.

Concerning the autoregressive parameter of the wild bootstrap, we can observe that whenever the data are serially correlated, the autoregressive wild bootstrap $(\gamma \neq 0)$ provides better coverage than the standard wild bootstrap $(\gamma=0)$. In addition, with stronger correlation, a larger value for $\gamma$ should be preferred, except that the case $\gamma=0.6$ provides consistently lower coverage, indicating that simply going for a very large value of $\gamma$ is not sensible in practice. However, even if we do see these patterns, in general, the coverage probabilities do not vary substantially with the autoregressive parameter and therefore, the sensitivity to this parameter appears to be fairly limited. 
When we look at the different blocks of Table 2.1, the bootstrap shows a similar overall performance regardless of the value we select for the bandwidth parameter. Since the bandwidth plays such an important role in nonparametric estimation, yet there are no fully satisfactory ways to select optimal bandwidth from the data in most applications, robustness to bandwidth "misspecifcation" is an important finding. This implies that the bootstrap can correct for poorly chosen bandwidths.

We observe similar patterns in Tables 2.3 and 2.2, while overall coverage is lower for the set $G$ than for $G_{s u b}$. This is not surprising, since the former set covers twice as many points as the latter. Interestingly, the confidence bands are consistently more narrow with $G$ than they are with $G_{s u b}$. This appears counterintuitive at first, as $G$ is twice as large as $G_{s u b}$. However, while $G_{s u b}$ is made up entirely of points relatively close to the boundaries, for which estimation is more variable, $G$ additionally contains "stable" regions closer to the center. It may be that the stability of these regions has an offsetting effect compared with the boundary regions, reducing the size of the intervals. As a side effect, overall coverage is also reduced. As such, if one is only interested in coverage near the beginning and the end of the sample, it may be wiser to only attempt to achieve uniformity over these regions, rather than over the full sample.

Similar to the pointwise coverage results, the simultaneous coverage is close to the nominal level for the case with negative correlation as well as the independent case. Weak positive correlation can also be handled decently. The cases $\phi=0.5$ and $\psi=0.5$ are more problematic, as coverage drops to around $60 \%$ for $G$ and $70 \%$ for $G_{s u b}$. In these cases, the smallest bandwidth $h=0.02$ seems to be preferred.

Comparing the AWB to the other two bootstrap methods, we can see that in almost all cases, AWB and DWB show similar results and they outperform the sieve version. Often, the AWB results in slightly higher coverage with shorter intervals. Only when $\gamma=0.6$, the DWB displays better coverage. Further increases of this parameter did not lead to improvements. An exception is the DGP with negative correlation, where the DWB displays coverage that is too high, independent of the choice of $\gamma$. In such cases, the AWB is often more accurate for $\gamma=0.4$. In all other cases, we see that for both methods, the best performance is similar in magnitude but obtained at a different value of the tuning parameter. Since the tuning parameters do not have exactly the same meaning in both methods, there is no reason to expect identical variation. We chose $\theta=0.01$ to link the two methods; changing this value will likely change the relation between the methods as well. 


\begin{tabular}{|c|c|c|c|c|c|}
\hline \multirow[b]{2}{*}{$h$} & \multirow[b]{2}{*}{ DGP } & \multirow{2}{*}{$\frac{-}{\mathrm{SWB}}$} & \multirow{2}{*}{$\begin{array}{c}\gamma=0 \\
\mathrm{WB}\end{array}$} & \multicolumn{2}{|c|}{$\gamma=0.2$} \\
\hline & & & & AWB & DWB \\
\hline \multirow{5}{*}{0.02} & 0 & $0.925(0.719)$ & $0.946(0.732)$ & $0.952(0.715)$ & $0.946(0.733)$ \\
\hline & $A R_{0.2}$ & $0.861(0.688)$ & $0.902(0.710)$ & $0.918(0.711)$ & $0.902(0.711)$ \\
\hline & $A R_{0.5}$ & $0.672(0.536)$ & $0.778(0.604)$ & $0.818(0.630)$ & $0.776(0.605)$ \\
\hline & $A R_{-0.5}$ & $0.960(0.552)$ & $0.989(0.623)$ & $0.988(0.575)$ & $0.989(0.624)$ \\
\hline & $M A_{0.2}$ & $0.872(0.690)$ & $0.910(0.712)$ & $0.925(0.712)$ & $0.911(0.712)$ \\
\hline \multirow{5}{*}{0.04} & 0 & $0.922(0.548)$ & $0.950(0.567)$ & $0.952(0.561)$ & $0.951(0.568)$ \\
\hline & $A R_{0.2}$ & $0.862(0.525)$ & $0.903(0.553)$ & $0.911(0.563)$ & $0.901(0.553)$ \\
\hline & $A R_{0.5}$ & $0.667(0.409)$ & $0.768(0.478)$ & $0.803(0.511)$ & $0.766(0.479)$ \\
\hline & $A R_{-0.5}$ & $0.970(0.423)$ & $0.996(0.492)$ & $0.994(0.457)$ & $0.996(0.492)$ \\
\hline & $M A_{0.2}$ & $0.873(0.526)$ & $0.912(0.553)$ & $0.922(0.563)$ & $0.913(0.554)$ \\
\hline \multirow{5}{*}{0.06} & 0 & $0.925(0.465)$ & $0.955(0.492)$ & $0.957(0.492)$ & $0.956(0.493)$ \\
\hline & $A R_{0.2}$ & $0.865(0.444)$ & $0.910(0.480)$ & $0.917(0.494)$ & $0.907(0.481)$ \\
\hline & $A R_{0.5}$ & $0.669(0.347)$ & $0.781(0.420)$ & $0.814(0.455)$ & $0.777(0.421)$ \\
\hline & $A R_{-0.5}$ & $0.979(0.358)$ & $0.997(0.432)$ & $0.996(0.408)$ & $0.997(0.432)$ \\
\hline & $M A_{0.2}$ & $0.875(0.446)$ & $0.918(0.480)$ & $0.928(0.495)$ & $0.920(0.481)$ \\
\hline & \multirow[b]{2}{*}{ DGP } & \multicolumn{2}{|c|}{$\gamma=0.4$} & \multicolumn{2}{|c|}{$\gamma=0.6$} \\
\hline & & AWB & DWB & AWB & DWB \\
\hline \multirow{5}{*}{0.02} & 0 & $0.942(0.658)$ & $0.945(0.733)$ & $0.905(0.555)$ & $0.945(0.733)$ \\
\hline & $A R_{0.2}$ & $0.912(0.672)$ & $0.902(0.711)$ & $0.871(0.582)$ & $0.902(0.711)$ \\
\hline & $A R_{0.5}$ & $0.828(0.621)$ & $0.778(0.604)$ & $0.791(0.562)$ & $0.777(0.603)$ \\
\hline & $A R_{-0.5}$ & $0.982(0.499)$ & $0.989(0.624)$ & $0.958(0.397)$ & $0.989(0.624)$ \\
\hline & $M A_{0.2}$ & $0.918(0.671)$ & $0.909(0.712)$ & $0.879(0.580)$ & $0.910(0.712)$ \\
\hline \multirow{5}{*}{0.04} & 0 & $0.940(0.527)$ & $0.951(0.568)$ & $0.905(0.460)$ & $0.951(0.568)$ \\
\hline & $A R_{0.2}$ & $0.905(0.544)$ & $0.901(0.553)$ & $0.872(0.487)$ & $0.902(0.554)$ \\
\hline & $A R_{0.5}$ & $0.818(0.519)$ & $0.767(0.478)$ & $0.795(0.489)$ & $0.765(0.477)$ \\
\hline & $A R_{-0.5}$ & $0.989(0.405)$ & $0.996(0.492)$ & $0.969(0.338)$ & $0.996(0.493)$ \\
\hline & $M A_{0.2}$ & $0.913(0.542)$ & $0.912(0.554)$ & $0.880(0.485)$ & $0.912(0.554)$ \\
\hline \multirow{5}{*}{0.06} & 0 & $0.946(0.471)$ & $0.956(0.493)$ & $0.917(0.422)$ & $0.956(0.493)$ \\
\hline & $A R_{0.2}$ & $0.913(0.486)$ & $0.907(0.480)$ & $0.887(0.447)$ & $0.909(0.481)$ \\
\hline & $A R_{0.5}$ & $0.831(0.468)$ & $0.779(0.420)$ & $0.815(0.453)$ & $0.776(0.420)$ \\
\hline & $A R_{-0.5}$ & $0.992(0.373)$ & $0.997(0.432)$ & $0.977(0.325)$ & $0.997(0.433)$ \\
\hline & $M A_{0.2}$ & $0.921(0.485)$ & $0.918(0.481)$ & $0.894(0.444)$ & $0.918(0.481)$ \\
\hline
\end{tabular}

Table 2.1: Pointwise coverage probabilities (average median interval length) for $k=4$ and $a=0.5$. 


\begin{tabular}{|c|c|c|c|c|c|}
\hline \multirow[b]{2}{*}{$h$} & \multirow[b]{2}{*}{ DGP } & \multirow{2}{*}{$\frac{-}{\mathrm{SWB}}$} & \multirow{2}{*}{$\begin{array}{c}\gamma=0 \\
\mathrm{WB}\end{array}$} & \multicolumn{2}{|c|}{$\gamma=0.2$} \\
\hline & & & & AWB & DWB \\
\hline \multirow{5}{*}{0.02} & 0 & $0.917(0.661)$ & $0.941(0.672)$ & $0.944(0.656)$ & $0.936(0.673)$ \\
\hline & $A R_{0.2}$ & $0.805(0.632)$ & $0.884(0.652)$ & $0.908(0.653)$ & $0.883(0.653)$ \\
\hline & $A R_{0.5}$ & $0.427(0.493)$ & $0.700(0.555)$ & $0.759(0.578)$ & $0.689(0.556)$ \\
\hline & $A R_{-0.5}$ & $0.985(0.507)$ & $0.982(0.572)$ & $0.981(0.528)$ & $0.983(0.573)$ \\
\hline & $M A_{0.2}$ & $0.820(0.634)$ & $0.899(0.654)$ & $0.913(0.653)$ & $0.891(0.654)$ \\
\hline \multirow{5}{*}{0.04} & 0 & $0.902(0.504)$ & $0.930(0.521)$ & $0.931(0.516)$ & $0.929(0.522)$ \\
\hline & $A R_{0.2}$ & $0.774(0.483)$ & $0.849(0.508)$ & $0.858(0.517)$ & $0.836(0.508)$ \\
\hline & $A R_{0.5}$ & $0.376(0.376)$ & $0.592(0.439)$ & $0.648(0.470)$ & $0.564(0.440)$ \\
\hline & $A R_{-0.5}$ & $0.993(0.390)$ & $0.997(0.452)$ & $0.991(0.420)$ & $0.995(0.453)$ \\
\hline & $M A_{0.2}$ & $0.790(0.483)$ & $0.859(0.508)$ & $0.877(0.517)$ & $0.857(0.509)$ \\
\hline \multirow{5}{*}{0.06} & 0 & $0.906(0.428)$ & $0.930(0.452)$ & $0.929(0.453)$ & $0.931(0.453)$ \\
\hline & $A R_{0.2}$ & $0.783(0.409)$ & $0.852(0.441)$ & $0.845(0.455)$ & $0.832(0.442)$ \\
\hline & $A R_{0.5}$ & $0.366(0.319)$ & $0.564(0.386)$ & $0.624(0.418)$ & $0.554(0.387)$ \\
\hline & $A R_{-0.5}$ & $0.995(0.330)$ & $0.998(0.397)$ & $0.995(0.376)$ & $0.998(0.398)$ \\
\hline & $M A_{0.2}$ & $0.796(0.410)$ & $0.862(0.442)$ & $0.870(0.455)$ & $0.857(0.443)$ \\
\hline & \multirow[b]{2}{*}{ DGP } & \multicolumn{2}{|c|}{$\gamma=0.4$} & \multicolumn{2}{|c|}{$\gamma=0.6$} \\
\hline & & AWB & DWB & AWB & DWB \\
\hline \multirow{5}{*}{0.02} & 0 & $0.934(0.604)$ & $0.935(0.672)$ & $0.870(0.510)$ & $0.937(0.673)$ \\
\hline & $A R_{0.2}$ & $0.888(0.617)$ & $0.879(0.653)$ & $0.822(0.534)$ & $0.884(0.653)$ \\
\hline & $A R_{0.5}$ & $0.769(0.570)$ & $0.700(0.555)$ & $0.687(0.515)$ & $0.700(0.553)$ \\
\hline & $A R_{-0.5}$ & $0.974(0.459)$ & $0.987(0.573)$ & $0.920(0.365)$ & $0.984(0.573)$ \\
\hline & $M A_{0.2}$ & $0.900(0.616)$ & $0.888(0.654)$ & $0.835(0.533)$ & $0.895(0.654)$ \\
\hline \multirow{5}{*}{0.04} & 0 & $0.907(0.484)$ & $0.932(0.522)$ & $0.818(0.423)$ & $0.933(0.522)$ \\
\hline & $A R_{0.2}$ & $0.834(0.500)$ & $0.835(0.508)$ & $0.759(0.448)$ & $0.848(0.509)$ \\
\hline & $A R_{0.5}$ & $0.685(0.476)$ & $0.577(0.440)$ & $0.604(0.449)$ & $0.573(0.439)$ \\
\hline & $A R_{-0.5}$ & $0.980(0.373)$ & $0.994(0.452)$ & $0.917(0.310)$ & $0.995(0.453)$ \\
\hline & $M A_{0.2}$ & $0.860(0.498)$ & $0.864(0.509)$ & $0.774(0.446)$ & $0.863(0.510)$ \\
\hline \multirow{5}{*}{0.06} & 0 & $0.907(0.433)$ & $0.939(0.453)$ & $0.819(0.388)$ & $0.936(0.453)$ \\
\hline & $A R_{0.2}$ & $0.835(0.447)$ & $0.840(0.442)$ & $0.757(0.411)$ & $0.851(0.442)$ \\
\hline & $A R_{0.5}$ & $0.663(0.431)$ & $0.565(0.387)$ & $0.594(0.416)$ & $0.537(0.386)$ \\
\hline & $A R_{-0.5}$ & $0.987(0.343)$ & $0.998(0.397)$ & $0.937(0.299)$ & $0.998(0.398)$ \\
\hline & $M A_{0.2}$ & $0.856(0.446)$ & $0.865(0.443)$ & $0.771(0.409)$ & $0.862(0.443)$ \\
\hline
\end{tabular}

Table 2.2: Simultaneous coverage probabilities (average median interval length) over $G$ for $k=4$ and $a=0.5$. 


\begin{tabular}{|c|c|c|c|c|c|}
\hline \multirow[b]{2}{*}{$h$} & \multirow[b]{2}{*}{ DGP } & \multirow{2}{*}{$\frac{-}{\text { SWB }}$} & \multirow{2}{*}{$\begin{array}{c}\gamma=0 \\
\mathrm{WB}\end{array}$} & \multicolumn{2}{|c|}{$\gamma=0.2$} \\
\hline & & & & AWB & DWB \\
\hline \multirow{5}{*}{0.02} & 0 & $0.894(0.518)$ & $0.925(0.527)$ & $0.939(0.515)$ & $0.925(0.528)$ \\
\hline & $A R_{0.2}$ & $0.738(0.496)$ & $0.855(0.512)$ & $0.887(0.513)$ & $0.855(0.512)$ \\
\hline & $A R_{0.5}$ & $0.266(0.386)$ & $0.608(0.435)$ & $0.690(0.454)$ & $0.604(0.435)$ \\
\hline & $A R_{-0.5}$ & $0.984(0.398)$ & $0.982(0.448)$ & $0.979(0.413)$ & $0.979(0.449)$ \\
\hline & $M A_{0.2}$ & $0.770(0.497)$ & $0.874(0.513)$ & $0.896(0.513)$ & $0.869(0.513)$ \\
\hline \multirow{5}{*}{0.04} & 0 & $0.875(0.388)$ & $0.914(0.401)$ & $0.915(0.397)$ & $0.915(0.402)$ \\
\hline & $A R_{0.2}$ & $0.697(0.372)$ & $0.812(0.391)$ & $0.822(0.398)$ & $0.793(0.391)$ \\
\hline & $A R_{0.5}$ & $0.206(0.289)$ & $0.461(0.338)$ & $0.544(0.362)$ & $0.439(0.339)$ \\
\hline & $A R_{-0.5}$ & $0.993(0.300)$ & $0.996(0.348)$ & $0.991(0.323)$ & $0.995(0.348)$ \\
\hline & $M A_{0.2}$ & $0.718(0.372)$ & $0.824(0.391)$ & $0.848(0.398)$ & $0.824(0.392)$ \\
\hline \multirow{5}{*}{0.06} & 0 & $0.884(0.327)$ & $0.913(0.345)$ & $0.911(0.346)$ & $0.912(0.346)$ \\
\hline & $A R_{0.2}$ & $0.705(0.313)$ & $0.794(0.337)$ & $0.804(0.348)$ & $0.781(0.338)$ \\
\hline & $A R_{0.5}$ & $0.196(0.244)$ & $0.415(0.295)$ & $0.497(0.320)$ & $0.395(0.296)$ \\
\hline & $A R_{-0.5}$ & $0.996(0.252)$ & $0.999(0.304)$ & $0.995(0.287)$ & $0.997(0.304)$ \\
\hline & $M A_{0.2}$ & $0.740(0.313)$ & $0.815(0.338)$ & $0.835(0.348)$ & $0.817(0.339)$ \\
\hline & \multirow[b]{2}{*}{ DGP } & \multicolumn{2}{|c|}{$\gamma=0.4$} & \multicolumn{2}{|c|}{$\gamma=0.6$} \\
\hline & & AWB & DWB & AWB & DWB \\
\hline \multirow{5}{*}{0.02} & 0 & $0.919(0.474)$ & $0.926(0.528)$ & $0.845(0.400)$ & $0.925(0.528)$ \\
\hline & $A R_{0.2}$ & $0.863(0.485)$ & $0.853(0.512)$ & $0.768(0.420)$ & $0.866(0.513)$ \\
\hline & $A R_{0.5}$ & $0.691(0.449)$ & $0.602(0.435)$ & $0.590(0.407)$ & $0.606(0.434)$ \\
\hline & $A R_{-0.5}$ & $0.972(0.359)$ & $0.986(0.449)$ & $0.915(0.285)$ & $0.984(0.449)$ \\
\hline & $M A_{0.2}$ & $0.876(0.484)$ & $0.865(0.513)$ & $0.792(0.419)$ & $0.870(0.513)$ \\
\hline \multirow{5}{*}{0.04} & 0 & $0.882(0.373)$ & $0.917(0.402)$ & $0.762(0.326)$ & $0.917(0.402)$ \\
\hline & $A R_{0.2}$ & $0.798(0.385)$ & $0.799(0.391)$ & $0.688(0.345)$ & $0.806(0.392)$ \\
\hline & $A R_{0.5}$ & $0.571(0.368)$ & $0.435(0.338)$ & $0.480(0.347)$ & $0.441(0.338)$ \\
\hline & $A R_{-0.5}$ & $0.981(0.287)$ & $0.995(0.348)$ & $0.907(0.239)$ & $0.995(0.349)$ \\
\hline & $M A_{0.2}$ & $0.818(0.384)$ & $0.824(0.392)$ & $0.705(0.344)$ & $0.832(0.392)$ \\
\hline \multirow{5}{*}{0.06} & 0 & $0.881(0.331)$ & $0.922(0.346)$ & $0.761(0.297)$ & $0.922(0.346)$ \\
\hline & $A R_{0.2}$ & $0.776(0.342)$ & $0.784(0.338)$ & $0.663(0.315)$ & $0.790(0.338)$ \\
\hline & $A R_{0.5}$ & $0.529(0.330)$ & $0.396(0.295)$ & $0.450(0.319)$ & $0.390(0.295)$ \\
\hline & $A R_{-0.5}$ & $0.988(0.262)$ & $0.998(0.304)$ & $0.932(0.228)$ & $0.998(0.304)$ \\
\hline & $M A_{0.2}$ & $0.807(0.341)$ & $0.827(0.338)$ & $0.682(0.313)$ & $0.826(0.338)$ \\
\hline
\end{tabular}

Table 2.3: Simultaneous coverage probabilities (average median interval length) over $G_{s u b}$ for $k=4$ and $a=0.5$. 
Next, we consider the setting with missing data. Given the previous findings, we restrict ourselves to one bandwidth $(h=0.06)$ but consider all AR and MA models. The results for pointwise as well as simultaneous coverage probabilities are given in Table 2.4; further results, with similar conclusions, are available in Supplementary Appendix C.

The first block presents pointwise coverage, while the second and third blocks show results for the sets $G_{s u b}$ and $G$. The AWB performs well even if a significant proportion of the data are missing, as both pointwise and simultaneous coverage is close to the nominal level for almost all models. There is a significant increase in pointwise coverage for the cases with strong positive correlation, which is now close to $90 \%$. The same increase is visible for both $G_{\text {sub }}$ and $G$. This phenomenon does not come as a surprise, as the missing data points create space between consecutive observations, thus effectively reducing the serial dependence between observed points. Comparing the AWB with the DWB, we can see that the coverage is slightly closer to $95 \%$ for the AWB in many cases. As before, the best performance is obtained at different values of $\gamma$ for the AWB and the DWB. The general pattern, however, is as in the previous setting. The DWB outperforms the AWB for higher values of $\gamma$, while the AWB obtains the most accurate coverage for smaller values of this parameter. A notable exception is again the DGP with negative autocorrelation. Results for other bandwidths are similar, and presented in Supplementary Appendix C.

Overall, this simulation study indicates that the autoregressive wild bootstrap performs well in most of our considered scenarios. The pointwise confidence intervals show coverage close to the nominal level in the presence of heteroskedasticity and serial correlation. In addition, the method still performs well in the presence of missing data. The AWB provides simultaneous confidence bands with good coverage as long as the correlation does not become too strong. It outperforms the sieve wild bootstrap whenever we could compare results. In comparison to the dependent wild bootstrap, we saw that both methods provide very similar coverage probabilities, while the DWB produces slightly wider intervals.

\subsection{Trends in Atmospheric Ethane}

We use our methodology to investigate the trending behavior of a time series of atmospheric ethane emissions which is derived from observations performed at the Jungfraujoch station in the Swiss Alps. This station can be found on the 


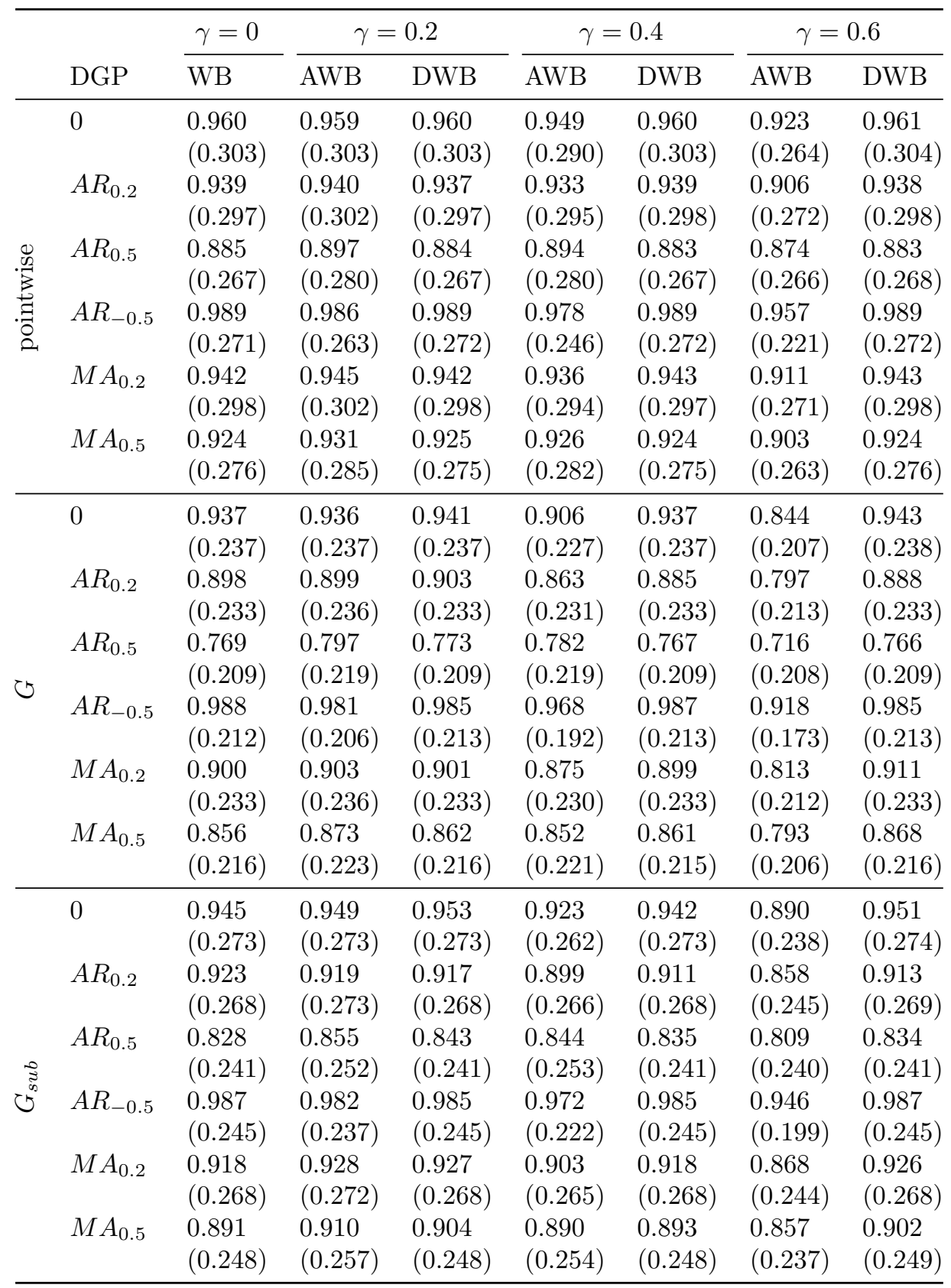

Table 2.4: Coverage probabilities (average median interval length) with missing data $(h=0.06)$. 
saddle between the Jungfrau and the Mönch, located at $46.55^{\circ} \mathrm{N}, 7.98^{\circ} \mathrm{E}, 3580 \mathrm{~m}$ altitude. Ethane is the most abundant hydrocarbon gas in the atmosphere after methane and it is used as a measure of atmospheric pollution. It contributes to the formation of ground-level ozone and it influences the lifetime of methane which classifies it as an indirect greenhouse gas. This series, which has been studied by Franco et al. (2015), is available from the Network for the Detection of Atmospheric Composition Change website. ${ }^{4}$

It is argued in Franco et al. (2015) that the measurement conditions are very favorable at the Jungfraujoch location due to high dryness and low local pollution. Further details on the ground-based station and on how the measurements are obtained can be found in the aforementioned reference. It is a time series consisting of daily ethane columns (i.e. the number of molecules integrated between the ground and the top of the atmosphere) recorded under clear-sky conditions between September 1994 and August 2014 with a total of 2260 data points. Whenever more than one measurement is taken on one day, a daily mean is considered.

The average number of data points per year is 112.6 - giving an indication of the severity of the missing data problem present in this series. This shows that, in line with the above discussion, it is of major importance to use a bootstrap method which can replicate the missing data pattern correctly. The estimated transition probabilities of a first order Markov Chain reported in (2.14) already indicated the presence of (weak) serial dependence in the missing data generating mechanism. As a further exploration of this mechanism, note that the local constant estimator implicitly provides an estimator for the smoothly varying proportion of non-missing data $p(\tau)$. We can write $(2.2)$ as

$$
\hat{m}(\tau)=\hat{p}(\tau)^{-1} \frac{1}{n h} \sum_{t=1}^{n} K\left(\frac{t / n-\tau}{h}\right) D_{t} y_{t},
$$

where

$$
\hat{p}(\tau)=\frac{1}{n h} \sum_{t=1}^{n} K\left(\frac{t / n-\tau}{h}\right) D_{t},
$$

and $\hat{p}(\tau)$ can be seen as an estimator of $p(\tau) .^{5}$ In Figure 2.2, we plot $\hat{p}(\cdot)$ as a diagnostic tool to investigate how data availability evolves over time. It fluctuates around the average proportion of 0.3 , with a maximum of almost 0.4 and a mini-

\footnotetext{
${ }^{4}$ Available at ftp://ftp.cpc.ncep.noaa.gov/ndacc/station/jungfrau/hdf/ftir/

${ }^{5}$ Lemma 2.4 establishes the consistency of this estimator.
} 
mum of 0.2 . Although no overall trend appears to be present, the fluctuations are serious enough to cast doubt on the stationarity of the missing data generating mechanism; however, our method can handle this without problems.

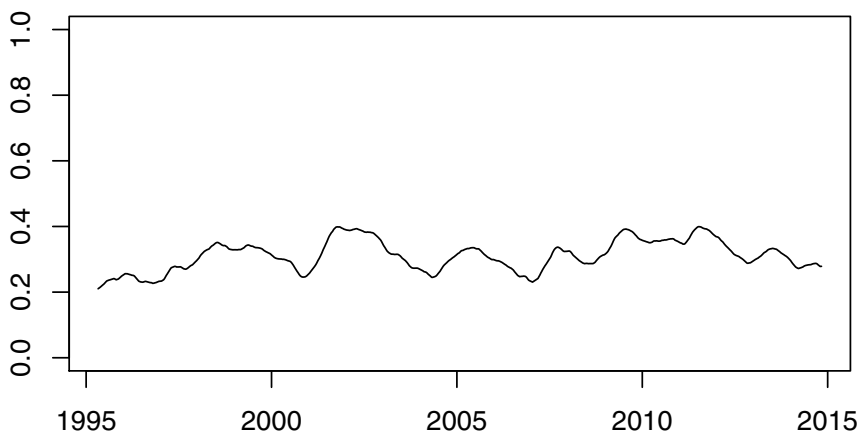

Figure 2.2: Time-varying proportion of non-missing data $\hat{p}$

In addition, the data exhibit strong seasonality, as ethane degrades faster in summer than it does in winter, causing the series to display peaks during winter and troughs during summer. Franco et al. (2015) take care of this seasonality with the help of Fourier terms by fitting the following model to ethane measurements $x_{t}$ :

$$
x_{t}=\sum_{j=1}^{M} a_{j} \cos (2 j \pi t)+b_{j} \sin (2 j \pi t)+y_{t} .
$$

They continue their analysis with the residuals from this estimation, where $M=3$ is selected by inspecting the residual variance. To investigate the sensitivity of the choice of $M$, we perform a frequency domain analysis to give more insight about the form of the periodic pattern present in our data. Due to the missing data, we use the Lomb-Scargle periodogram, which is suitable in this situation (see Lomb (1976); Scargle (1982)). Figure 2.3 plots the periodogram of the Jungfraujoch series with the frequency, rescaled to years, on the horizontal axis.

The peaks around zero are the smooth long-run trend we model with our trend estimator. The present seasonality induces additional peaks at higher frequencies. There is a large peak at 1 , representing an annual periodicity, which is so pronounced that it obscures peaks at other frequencies. Therefore, the right panel displays the same spectrum as the left panel, but with a smaller vertical axis such 


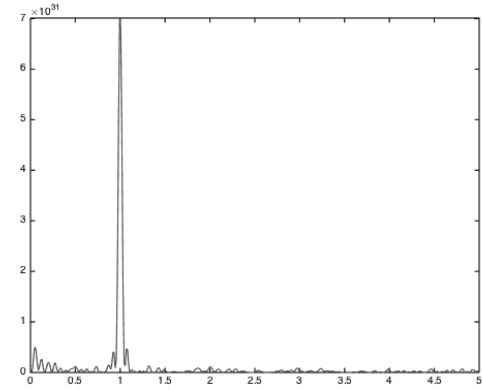

(a) Full periodogram

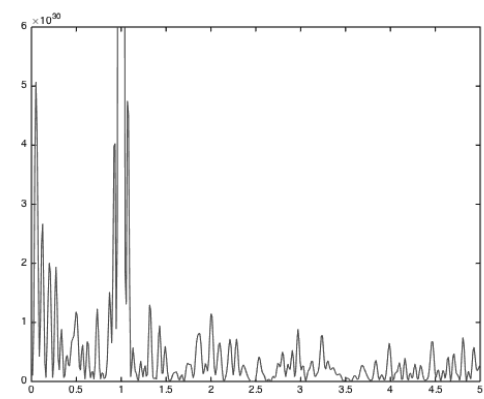

(b) Zoomed version

Figure 2.3: The Lomb-Scargle periodogram of the ethane series

that other peaks are observed more clearly. Moreover, we can observe that there are peaks at 2 and 3. They are, however, not as clear-cut as the peak at 1 and might not contribute as much to the seasonality, yet provide further justification for the choice $M=3$. In Supplementary Appendix D, we consider the periodograms of the residuals of the regression on 1 up to 4 Fourier terms. These show that while inclusion of one term is clearly needed, including more terms does indeed further reduce periodicity, although for increasing $M$, the effect becomes less pronounced.

To corroborate our results and to be able to compare our findings to Franco et al. (2015), we additionally look at the Akaike and Bayesian information criteria as well as the residual variance (MSE in Franco et al. (2015)) from the above regression for different values of $M$. The results are summarized in Table 2.5. While the Akaike criterion (AIC) is indifferent between adding 4 or 5 Fourier terms, BIC selects 2. The residual variance (MSE) decreases by only small increments when more than 3 terms are included. Based on our analysis, it is not clear how many Fourier terms we should include; any value of $M$ between 1 and 4 seems reasonable. In the following, we report results for applying the trend estimation on the residuals of the regression with $M=3$ Fourier terms, as in Franco et al. (2015). In Supplementary Appendix D we perform the same analysis with $M$ varying between 1 and 4, with hardly any difference in the results.

We next investigate bandwidth selection. As suggested in Section 2.3.1, we determine a possible bandwidth using modified cross-validation. In line with the discussion in Chu and Marron (1991), for our series, the ordinary leave-one-out cross-validation criterion selects a bandwidth which is too small $\left(h_{c v}=0.0006\right)$. 


\begin{tabular}{|c|c|c|c|}
\hline$M$ & $A I C$ & $B I C$ & $M S E$ \\
\hline 1 & 158447.5 & 158464.6 & $2.79909 \times 10^{30}$ \\
\hline 2 & 158420.0 & $158448.6^{*}$ & $2.76038 \times 10^{30}$ \\
\hline 3 & 158413.4 & 158453.5 & $2.74750 \times 10^{30}$ \\
\hline 4 & $158411.3^{*}$ & 158462.8 & $2.74010 \times 10^{30}$ \\
\hline 5 & $158411.3^{*}$ & 158474.3 & $2.73521 \times 10^{30}$ \\
\hline 6 & 158413.7 & 158488.1 & $2.73329 \times 10^{30}$ \\
\hline 7 & 158416.8 & 158502.7 & $2.73219 \times 10^{30}$ \\
\hline
\end{tabular}

Table 2.5: Fourier term investigation

This value for the bandwidth parameter gives almost no smoothing of the data and the resulting trend curve is too wiggly. Leaving out $k=5$ observations on each side of any point, the modified criterion yields a value of $h_{m c v}=0.03$. Albeit a still small bandwidth, this value gives a much more reasonable picture of the trend estimate. The resulting nonparametric estimate as well as $95 \%$ simultaneous confidence bands are depicted in Figure 2.4. The confidence bands are simultaneous over the whole sample. Although the validity has not been established, the algorithm works when we cover the whole sample and the results are easier to interpret. The bands are obtained using $B=999$ replications of the bootstrap procedure and an autoregressive parameter of $\gamma=0.5$.

As a robustness check, we also applied the trend estimation to the data without explicitly modeling the seasonality using Fourier terms. The nonparametric kernel estimator can be interpreted as a low pass filter which suppresses high frequency oscillations. A sufficiently large bandwidth should introduce enough smoothing to provide a trend curve which is not driven by the seasonal component. The bandwidth selected by MCV is too small for this effect to appear in our data. When we increase the bandwidth to $h=0.06$, the resulting trend shows the same pattern as the one in Figure 2.4. This analysis shows that when strong seasonality is present in the data, the nonparametric kernel estimator can be used to filter out the seasonality if the bandwidth is large enough. In this case, however, bandwidth selection becomes a critical issue and the proposed MCV criterion should be applied with care. Further details can be found in Supplementary Appendix D.

We observe a slight downward trend of the ethane time series until around 2009, with local peaks in 1998 and 2002-2003, and an upward trend thereafter. This general development of the trend supports the findings in Franco et al. (2015) 


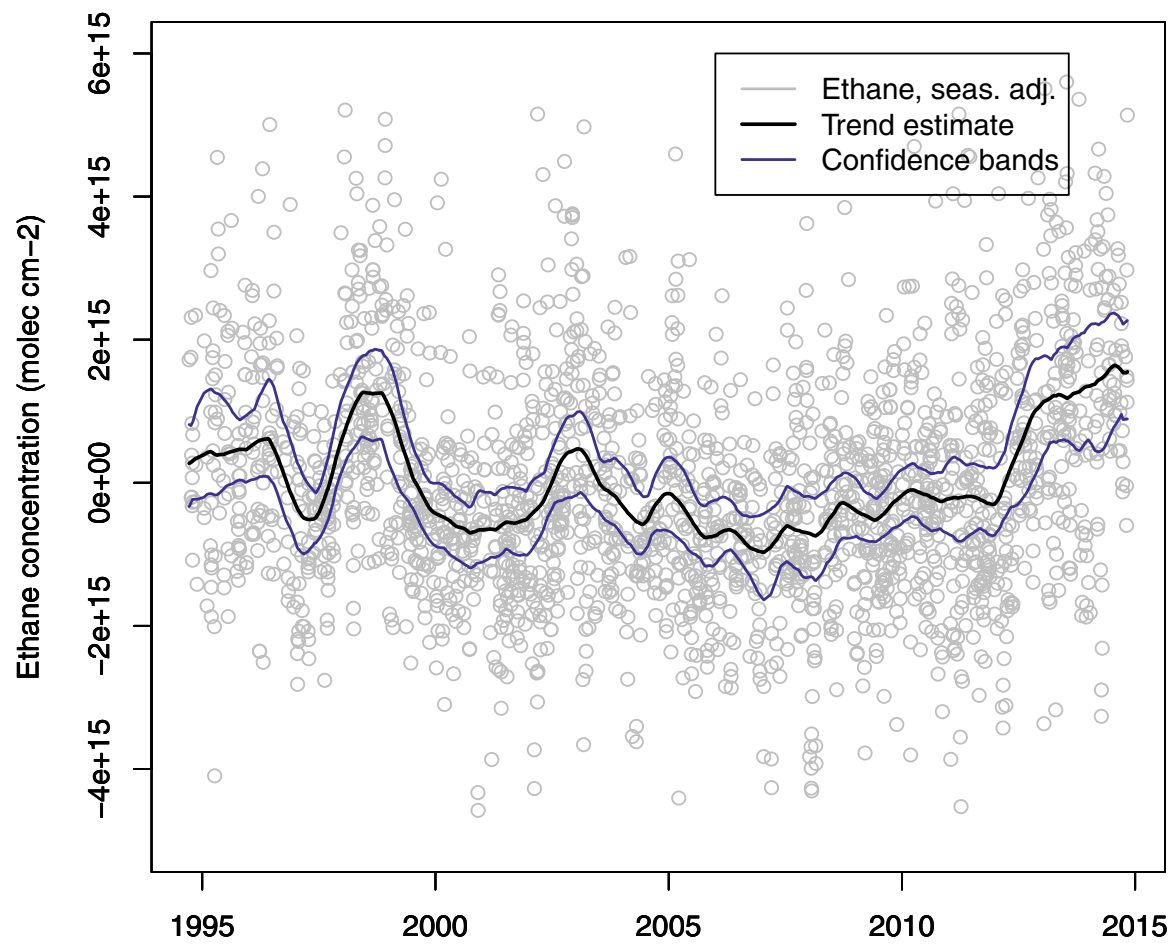

Figure 2.4: Trend estimate with uniform 95\% confidence bands for ethane concentration at the Jungfraujoch (with bandwidth selected by MCV).

who estimate a linear trend model with a break at the beginning of 2009 . They find a negative slope of the trend line before the break and a positive slope after the break. As mentioned by Franco et al. (2015), the initial downward trend can be explained by a general emission reduction since the mid 1980s, of the fossil fuel sources in the Northern Hemisphere. This has also been reported by Simpson et al. (2012). The upward trend seems to be a more recent phenomenon. Studies attribute it to the recent growth in the exploitation of shale gas and tight oil reservoirs, taking place in North America, see e.g. Vinciguerra et al. (2015) and Franco et al. (2016). Since previous studies have mainly used methods based on linear trends, the two local peaks have to our knowledge not yet been analyzed. They can potentially be explained by boreal forest fires which were taking place mainly in Russia during both periods. Geophysical studies have investigated these events in association with anomalies in carbon monoxide emissions (Yurganov 
et al., 2004, 2005). In such fires, carbon monoxide is co-emitted with ethane, such that these events are likely explanations for the peaks we observe.

As a final step, we look at the standard deviation of the residuals. When estimating it with a nonparametric kernel smoother, we see a cyclical pattern with upward trend, similar to the process we generate in our simulations. We plot the estimated standard deviation in Figure 2.5. This clearly shows that the residuals are heteroskedastic which further underlines the importance of a flexible bootstrap method.

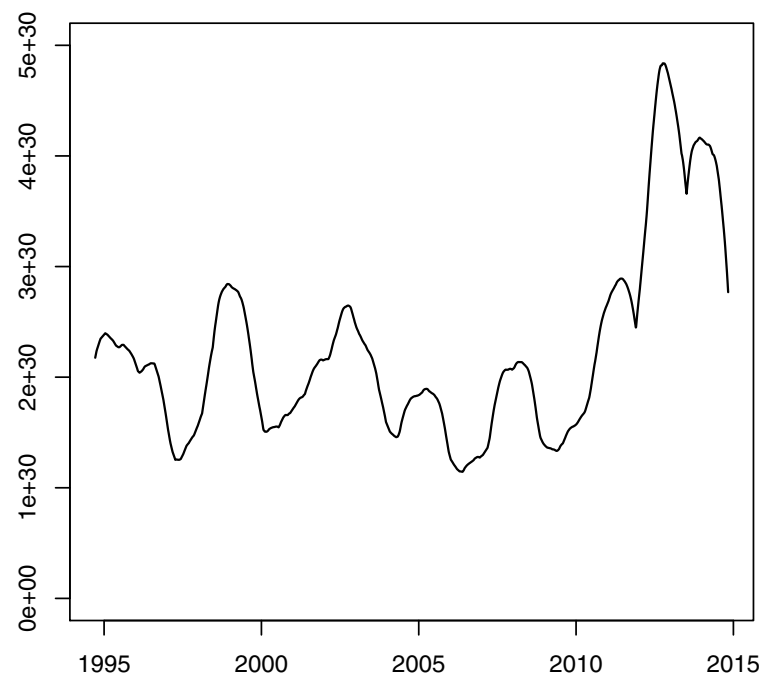

Figure 2.5: Estimate of the standard deviation of the residuals, obtained using the local constant kernel smoother with Epanechnikov kernel and $h=0.04$

\subsection{Conclusion}

In this chapter, we have proposed a dependent version of the wild bootstrap, the autoregressive wild bootstrap, to construct confidence intervals around a nonparametrically estimated trend. Consistency of the bootstrap has been established such that it can be used to construct pointwise and simultaneous confidence bands. While the pointwise intervals always show good coverage in finite samples, simulation results for the simultaneous bands indicate that strong positive autocorrelation leads to a drop in coverage whenever simultaneous confidence bands 
are considered. However, other bootstrap methods such as the dependent wild bootstrap and the sieve bootstrap are equally affected, and overall the autoregressive wild bootstrap performs at least on par with these other methods, and often outperforms them.

One major advantage of the proposed approach is its broad applicability as it can be used under general forms of serial dependence and heteroskedasticity. Furthermore, it can be applied without further adjustments when data points are missing. This feature of the autoregressive wild bootstrap is particularly relevant in economic and climatological applications where the problem of missing data is often encountered. In addition to simulation results, we provide a rigorous asymptotic analysis where asymptotically pervasive missing data are allowed for. While the missing data generating mechanism affects the asymptotic distribution of the estimator, our bootstrap method correctly mimics this and is therefore valid in the presence of general forms of missing data patterns.

An application to atmospheric ethane measurements from Switzerland demonstrates our methodology. An upward trend in this time series is an indication of increasing atmospheric pollution and it has been visible in the data for the last quarter. This finding is in line with previous studies in geophysics and provides further evidence that an increased activity in shale gas extraction might have caused an increase in the ethane burden measured over the Jungfraujoch. In addition, we find two local peaks in the ethane series, which can be explained by boreal forest fires. Natural limitations of linear trend estimation have prevented these peaks from being discovered in previous research. This underlines the flexibility of our approach compared to parametric methods.

An open end to our analysis is the choice of the autoregressive parameter in the autoregressive wild bootstrap. Although our simulation results suggest that a range of values for this parameter perform adequately, its selection in practice remains an open issue. Theoretical results on the choice of this parameter are not trivial; moreover, such theoretical results do not translate directly into selection methods with good properties in small samples. This issue therefore merits deeper study and is left as an exercise for future research. 


\section{A Technical Results}

For the remainder of this appendix, we define some further notation that will help lighten the notational load. Define $k_{t}(\tau)=K\left(\frac{t / n-\tau}{h}\right)$ and $\tilde{k}_{t}(\tau)=K\left(\frac{t / n-\tau}{\tilde{h}}\right)$. For any random variable $X$, let $\mathbb{E}_{D} X=\mathbb{E}\left(X \mid\left\{D_{t}\right\}_{t=1}^{n}\right)$ and $\operatorname{Var}_{D} X=\mathbb{E}_{D}[X-$ $\left.\left(\mathbb{E}_{D} X\right)^{2}\right]^{2}$. Furthermore, let $\|X\|_{p}=\left(\mathbb{E}|X|^{p}\right)^{1 / p}$ denote the $L_{p}$-norm of $X$, where we use $\|X\|$ as short-hand notation for $\|X\|_{2}$, and define the bootstrap equivalent as $\left\|X^{*}\right\|_{p}^{*}=\left(\mathbb{E}^{*}\left|X^{*}\right|^{p}\right)^{1 / p}$. Finally, $C, C_{1}, C_{2}, \ldots$ denote arbitrary fixed positive constants not depending on $n$ or $h$, whose value can change each line, while $\phi_{n}$ denotes a generic deterministic sequence with the property that $\lim _{n \rightarrow \infty} \phi_{n}=0$.

\section{A.1 Auxiliary lemmas}

We first establish some auxiliary lemmas with basic results that will be needed in the proofs of our main results. The proofs of these auxiliary lemmas are relegated to Supplementary Appendix B.

Lemma 2.1. Let Assumptions 2.3-2.4 hold. Then for all $n \geq 1$,
(i) $\sum_{i=0}^{n-1} i \max _{1 \leq t \leq n-i} \operatorname{Cov}\left(D_{t}, D_{t+i}\right) \leq C_{1}$;
(ii) $\sum_{i=0}^{n-1} i \max _{1 \leq t \leq n-i} \operatorname{Cov}\left(u_{t}, u_{t+i}\right) \leq C_{2}$;
(iii) $\sum_{i=0}^{n-1} i \max _{1 \leq t \leq n-i} \operatorname{Cov}\left(D_{t} u_{t}, D_{t+i} u_{t+i}\right) \leq C_{3}$.

Proof of Lemma 2.1. See Supplementary Appendix B.

Lemma 2.2. Under Assumption 2.5, we have for any $\delta>0$ that

$$
\sup _{\tau \in[\delta, 1-\delta]} \max _{0 \leq i \leq n} \frac{1}{n h} \sum_{t=1}^{n-i} k_{t}(\tau) k_{t+i}(\tau)<\infty .
$$

Proof of Lemma 2.2. See Supplementary Appendix B.

Lemma 2.3. Let $f_{n}(\cdot):[0,1] \rightarrow \mathbb{R}$ satisfy Assumption 2.1, and let $g(\cdot, \cdot)$ : $[0,1]^{2} \rightarrow \mathbb{R}^{2}$ be a Lipschitz continuous function with Lipschitz constant $C_{g}=$ $\sup _{\left(\tau_{1}, \tau_{2}\right) \in[0,1]^{2}}\left|g_{n}\left(\tau_{1}, \tau_{2}\right)\right|$. Let Assumption 2.5 be satisfied. Then, there exists 
some $N>0$, such that for any $0<\delta<1 / 2$, all $n>N$ and all $i=0,1, \ldots, n$, we have that

$$
\begin{gathered}
\text { (i) } \sup _{\tau \in[\delta, 1-\delta]}\left|\frac{1}{n h} \sum_{t=1}^{n} f\left(\frac{t}{n}\right) k_{t}(\tau)-f(\tau) \kappa_{1}-\frac{1}{2} h^{2} f^{(2)}(\tau) \mu_{2}\right| \leq C \max \left\{h^{3}, \frac{1}{n h}\right\} ; \\
\text { (ii) } \sup _{\tau_{0} \in[\delta, 1-\delta]} \sup _{\tau_{1}, \tau_{2} \in[-1,1]} \mid \frac{1}{n h} \sum_{t=1}^{n-i} g\left(\frac{t}{n}, \frac{t+i}{n}\right) k_{t}\left(\tau_{0}+\tau_{1} h\right) k_{t+i}\left(\tau_{0}+\tau_{2} h\right) \\
\quad-g\left(\tau_{0}, \tau_{0}\right) \kappa\left(\tau_{1}-\tau_{2}\right) \mid \leq C_{g}\left[\frac{C_{1} i}{n h}+C_{2} \max \left\{h, \frac{1}{n h^{2}}\right\}+S_{n}(i)\right],
\end{gathered}
$$

where, for any sequence $\left\{\beta_{i}\right\}_{i=0}^{\infty}$ with $\sum_{i=1}^{\infty}\left|\beta_{i}\right|<\infty$, we have that $\sum_{i=1}^{\infty}\left|\beta_{i}\right| S_{n, i} \leq$ $\phi_{n}$, where $\lim _{n \rightarrow \infty} \phi_{n}=0$.

Proof of Lemma 2.3. See Supplementary Appendix B.

Lemma 2.4. Let Assumptions 2.2-2.5 be satisfied. Define

$$
Z_{n, D, f, q}(\tau)=\frac{1}{\sqrt{n h}} \sum_{t=1}^{n} k_{t}(\tau)\left[f\left(\frac{t}{n}\right)-q_{n}(\tau)\right]\left[D_{t}-p\left(\frac{t}{n}\right)\right]
$$

where $f(\cdot)$ satisfies Assumption 2.1 and $q_{n}(\cdot):[0,1] \rightarrow \mathbb{R}$ is a sequence of deterministic functions with $\sup _{\tau \in[0,1]}\left|q_{n}(\tau)\right|<\infty$ for all $n$. Then, there exists some $N>0$, such that for any $0<\delta<1 / 2$ and all $n>N$ the following hold:

(i) $\mathbb{E} Z_{n, D, f, q}(\tau)=0$ and

$$
\sup _{\tau \in[\delta, 1-\delta]}\left|\mathbb{E} Z_{n, D, f, l}(\tau)^{2}-\left[f(\tau)-q_{n}(\tau)\right]^{2} \Omega_{D}(\tau) \kappa_{2}\right| \leq C \max \left\{h, \frac{1}{n h^{2}}, \phi_{n}\right\},
$$

where $\Omega_{D}(\tau)=R_{D, 0}(\tau, \tau)+2 \sum_{i=1}^{\infty} R_{D, i}(\tau, \tau)$ and $\lim _{n \rightarrow \infty} \phi_{n}=0$.

(ii) For all $\tau \in(0,1)$,

$$
\frac{1}{n h} \sum_{t=1}^{n} f\left(\frac{t}{n}\right) k_{t}(\tau) D_{t}-p(\tau) f(\tau)-\frac{1}{2} h^{2}[f p]^{(2)}(\tau) \mu_{2}=\frac{Z_{n, D, f, 0}(\tau)}{\sqrt{n h}}+R_{n, D}(\tau),
$$

where $Z_{n, D, f, 0}(\tau)$ is defined as in (2.16) with $q_{n}(\cdot)=0$ and $\sup _{\tau \in[\delta, 1-\delta]}\left|R_{n, D}(\tau)\right|<$ $C \max \left\{h^{3}, \frac{1}{n h}\right\}$. 
(iii) Let $\tilde{g}_{i}(\cdot, \cdot)$ be a Lipschitz continuous function with Lipschitz constant $C_{g}(i)=\sup _{\left(\tau_{1}, \tau_{2}\right) \in[0,1]^{2}}\left|\tilde{g}_{i}\left(\tau_{1}, \tau_{2}\right)\right|$. Then for all $i=0,1, \ldots, n-1$,

$$
\begin{aligned}
& \sup _{\tau \in[\delta, 1-\delta]} \sup _{\tau_{1}, \tau_{2} \in[-1,1]} \mathbb{E} \mid \frac{1}{n h} \sum_{t=1}^{n} \tilde{g}_{i}\left(\frac{t}{n}, \frac{t+i}{n}\right) k_{t}\left(\tau_{0}+\tau_{1} h\right) k_{t+i}\left(\tau_{0}+\tau_{2} h\right) D_{t} D_{t+i} \\
& -p\left(\tau_{0}\right) \tilde{g}\left(\tau_{0}, \tau_{0}\right) \kappa\left(\tau_{1}-\tau_{2}\right) \mid \leq C_{g}(i)\left[\frac{C_{1}}{\sqrt{n h}}+C_{2} \max \left\{h, \frac{1}{n h^{2}}\right\}+S_{n}(i)\right],
\end{aligned}
$$

where $S_{n}(i)$ is defined in Lemma 2.3.

Proof of Lemma 2.4. See Supplementary Appendix B.

Lemma 2.5. Let Assumptions 2.1-2.6 hold. Then, for all $0 \leq i \leq n-1$ and any $0<\delta<1 / 2$,

$\sup _{\tau \in[\delta, 1-\delta]} \mathbb{E}\left|\frac{1}{n h} \sum_{t=1}^{n-i} k_{t}(\tau) k_{t+i}(\tau) \sigma_{t} \sigma_{t+i} D_{t} D_{t+i}\left[u_{t} u_{t+i}-\mathbb{E} u_{t} u_{t+i}\right]\right| \leq \beta_{i} \phi_{n}+\frac{\eta_{n}}{\sqrt{n h}}$,

where $\sum_{i=1}^{\infty} \beta_{i}<\infty, \lim _{n \rightarrow \infty} \phi_{n}=0$ and $\limsup _{n \rightarrow \infty} \eta_{n}<\infty$.

Proof of Lemma 2.5. See Supplementary Appendix B.

\section{A.2 Pointwise Results}

Lemmas 2.6 and 2.7 decompose the (bootstrap) estimator in the relevant components, establishing not only consistency but also providing the necessary building blocks towards asymptotic normality. We therefore present these lemmas and their proofs together with the proofs of Theorems 2.1 and 2.2 .

Lemma 2.6. Let Assumptions 2.1-2.6 be satisfied. Then, for some $N>0$ and any $0<\delta<1 / 2$, we have for all $\tau \in(0,1)$ and $n>N$ that

$$
\sqrt{n h}\left[\hat{m}(\tau)-m(\tau)-h^{2} B_{a s}(\tau)\right]=p(\tau)^{-1} Z_{n, U}(\tau)+R_{n}(\tau),
$$

where $B_{a s}(\tau)$ is defined in (2.8),

$$
\begin{aligned}
& Z_{n, U}(\tau)=\frac{1}{\sqrt{n h}} \sum_{t=1}^{n} k_{t}(\tau) D_{t} \sigma_{t} u_{t}, \\
& \sigma_{Z}^{2}(\tau)=\operatorname{plim}_{n \rightarrow \infty} \operatorname{Var}\left[Z_{n, U}(\tau) \mid\left\{D_{t}\right\}_{t=1}^{n}\right]=p(\tau) \sigma(\tau)^{2} \Omega_{U} \kappa_{2},
\end{aligned}
$$




$$
\sup _{\tau \in[\delta, 1-\delta]}\left\|R_{n}(\tau)\right\| \leq C \max \left\{h^{2}, \sqrt{n h^{7}}, \frac{1}{\sqrt{n h}}\right\} .
$$

Proof of Lemma 2.6. Defining

$$
\begin{aligned}
\hat{p}(\tau) & =\frac{1}{n h} \sum_{t=1}^{n} k_{t}(\tau) D_{t}, & \breve{m}(\tau) & =\frac{1}{n h} \sum_{t=1}^{n} k_{t}(\tau) D_{t} y_{t}, \\
\bar{m}_{D}(\tau) & =\frac{1}{n h} \sum_{t=1}^{n} k_{t}(\tau) D_{t} m(t / n) & \bar{m}_{p}(\tau) & =\frac{1}{n h} \sum_{t=1}^{n} k_{t}(\tau) p(t / n) m(t / n),
\end{aligned}
$$

and realizing that $Z_{n, U}(\tau)=\sqrt{n h}\left[\breve{m}(\tau)-\bar{m}_{D}(\tau)\right]$, we can write

$$
\begin{aligned}
\sqrt{n h}\left[\hat{m}(\tau)-m(\tau)-h^{2} B_{a s}(\tau)\right]= & p(\tau)^{-1} Z_{n, U}(\tau) \\
& +\left[\hat{p}(\tau)^{-1}-p(\tau)^{-1}\right] Z_{n, U}(\tau) / \sqrt{n h} \\
& +\sqrt{n h}\left\{\hat{p}(\tau)^{-1} \bar{m}_{D}(\tau)-p(\tau)^{-1} \bar{m}_{p}(\tau)\right\} \\
& +\sqrt{n h}\left\{p(\tau)^{-1} \bar{m}_{p}(\tau)-m(\tau)-h^{2} B_{a s}(\tau)\right\} \\
= & p(\tau)^{-1} Z_{n, U}(\tau)+I_{n}(\tau)+I I_{n}(\tau)+I I I_{n}(\tau) .
\end{aligned}
$$

We first derive $\sigma_{Z}^{2}(\tau)$. Let $\mathbb{E}_{D}(\cdot)=\mathbb{E}\left(\cdot \mid\left\{D_{t}\right\}_{t=1}^{n}\right)$. Then $\mathbb{E}_{D} Z_{n, U}(\tau)=0$ and

$$
\begin{aligned}
\mathbb{E}_{D} Z_{n, U}(\tau)^{2} & =\frac{1}{n h} \sum_{s=1}^{n} \sum_{t=1}^{n} k_{s}(\tau) k_{t}(\tau) D_{s} D_{t} \sigma_{s} \sigma_{t} \mathbb{E} u_{s} u_{t} \\
& =\frac{1}{n h} \sum_{i=-n+1}^{n-1} R_{U}(i) \sum_{t=1}^{n-|i|} k_{t}(\tau) k_{t+|i|}(\tau) \sigma_{t} \sigma_{t+|i|} D_{t} D_{t+|i|}
\end{aligned}
$$

Using Lemma 2.4(iii) with $\tilde{g}_{i}\left(\tau_{1}, \tau_{2}\right)=\sigma\left(\tau_{1}\right) \sigma\left(\tau_{2}\right)$, we find that

$$
\begin{aligned}
& \sup _{\tau \in[\delta, 1-\delta]} \mathbb{E} \mid \sum_{i=-n+1}^{n-1} R_{U}(i) \frac{1}{n h} \sum_{t=1}^{n-|i|} k_{t}(\tau) k_{t+|i|}(\tau) \sigma_{t} \sigma_{t+|i|} D_{t} D_{t+|i|} \\
& \quad-p(\tau) \sigma(\tau)^{2} \kappa_{2} \Omega_{U} \mid \\
& \leq 2 \sum_{i=0}^{n-1}\left|R_{U}(i)\right|\left[\frac{C_{1}}{\sqrt{n h}}+C_{2} \max \left\{h, \frac{1}{n h^{2}}\right\}+S_{n}(i)\right]+2 \sum_{i=n}^{\infty}\left|R_{U}(i)\right|, \\
& \leq \frac{C_{3}}{\sqrt{n h}}+C_{4} \max \left\{h, \frac{1}{n h^{2}}\right\}+C_{5} \phi_{n} .
\end{aligned}
$$


where $\lim _{n \rightarrow \infty} \phi_{n}=0$, from which it follows that, for $\tau \in(0,1)$,

$$
\mathbb{E}\left|\mathbb{E}_{D} Z_{n, U}(\tau)^{2}-\sigma_{Z}^{2}(\tau)\right| \leq C \max \left\{(n h)^{-1 / 2}, h, n^{-1} h^{2}, \phi_{n}\right\}=o(1),
$$

where $\sigma_{Z}^{2}(\tau)$ is defined in $(2.17 \mathrm{~b})$.

Furthermore, by the arguments used above it follows that $\sup _{\tau \in[\delta, 1-\delta]}\left\|Z_{n, U}(\tau)\right\| \leq$ $C$, while by Lemma $2.4(i)$

$$
\|\hat{p}(\tau)-p(\tau)\| \leq \frac{1}{\sqrt{n h}}\left\|Z_{n, D}(\tau)\right\|+C_{1} h^{2}+C_{2} \max \left\{h^{3}, \frac{1}{n h}\right\} \leq C_{3} \max \left\{\frac{1}{\sqrt{n h}}, h^{2}\right\} .
$$

By combining these results with the fact that $\|\hat{p}(\tau)\|^{-1} \leq 1 / \epsilon^{*}$ by Assumption 2.6, we find that

$$
\begin{aligned}
\sup _{\tau \in[\delta, 1-\delta]}\left\|I_{n}(\tau)\right\| & \leq \sup _{\tau \in[\delta, 1-\delta]}\|\hat{p}(\tau)-p(\tau)\|\left\|\hat{p}(\tau)^{-2}\right\|\left|p(\tau)^{-2}\right|\left\|Z_{n, U}(\tau)\right\| \\
& \leq C_{4} \max \left\{\frac{1}{\sqrt{n h}}, h^{2}\right\} .
\end{aligned}
$$

For $I I_{n}(\tau)$ we note that

$$
\hat{p}(\tau)^{-1}-p(\tau)^{-1}=p(\tau)^{-2}\{p(\tau)-\hat{p}(\tau)\}+\hat{p}(\tau)^{-1} p(\tau)^{-2}\{p(\tau)-\hat{p}(\tau)\}^{2},
$$

such that we can rewrite $I I_{n}(\tau)$ as

$$
\begin{aligned}
I I_{n}(\tau) / \sqrt{n h}= & p(\tau)^{-1}\left\{\bar{m}_{D}(\tau)-\bar{m}_{p}(\tau)\right\}+\left\{\hat{p}(\tau)^{-1}-p(\tau)^{-1}\right\} \bar{m}_{p}(\tau) \\
& +\left\{\hat{p}(\tau)^{-1}-p(\tau)^{-1}\right\}\left\{\bar{m}_{D}(\tau)-\bar{m}_{p}(\tau)\right\} \\
= & p(\tau)^{-1}\left\{\bar{m}_{D}(\tau)-\bar{m}_{p}(\tau)\right\}-p(\tau)^{-2}\{\hat{p}(\tau)-p(\tau)\} \bar{m}_{p}(\tau) \\
& +\hat{p}(\tau)^{-1} p(\tau)^{-2}\{\hat{p}(\tau)-p(\tau)\}^{2} \bar{m}_{p}(\tau)+\left\{\hat{p}(\tau)^{-1}-p(\tau)^{-1}\right\} \\
& \times\left\{\bar{m}_{D}(\tau)-\bar{m}_{p}(\tau)\right\} \\
= & {\left[I I_{n, 11}(\tau)-I I_{n, 12}(\tau)+I I_{n, 2}(\tau)+I I_{n, 3}(\tau)\right] / \sqrt{n h} . }
\end{aligned}
$$

As $I I_{n, 11}(\tau)=p(\tau)^{-1} \frac{1}{\sqrt{n h}} \sum_{t=1}^{n} k_{t}(\tau) m(t / n)\left[D_{t}-p(t / n)\right]$ and $I I_{n, 12}(\tau)=p(\tau)^{-2} \bar{m}_{p}(\tau) \frac{1}{\sqrt{n h}} \sum_{t=1}^{n} k_{t}(\tau) \times\left[D_{t}-p(t / n)\right]$, we can write

$$
\begin{aligned}
I I_{n, 1}(\tau) & =I I_{n, 11}(\tau)-I I_{n, 12}(\tau) \\
& =p(\tau)^{-1} \frac{1}{\sqrt{n h}} \sum_{t=1}^{n} k_{t}(\tau)\left[m(t / n)-p(\tau)^{-1} \bar{m}_{p}(\tau)\right]\left[D_{t}-p(t / n)\right]
\end{aligned}
$$




$$
=p(\tau)^{-1} Z_{n, D}(\tau)
$$

where $Z_{n, D}(\tau)$ is defined as $Z_{n, D, f, q}(\tau)$ in Lemma $2.4(i)$ with $f(\cdot)=m(\cdot)$ and $q_{n}(\cdot)=p(\cdot)^{-1} \bar{m}_{p}(\cdot)$. Applying this lemma we find that

$$
\begin{aligned}
& \sup _{\tau \in[\delta, 1-\delta]}\left|\mathbb{E} Z_{n, D}(\tau)^{2}-\left[m(\tau)-p(\tau)^{-1} \bar{m}_{p}(\tau)\right]^{2} \Omega_{D}(\tau) \kappa_{2}\right| \\
& \leq C \max \left\{h, n^{-1} h^{2}\right\}+\phi_{n}
\end{aligned}
$$

As $\left|x^{2}-y^{2}\right| \leq|x-y|(|x-y|+2|y|)$, it follows from Lemma 2.3(i) that

$$
\begin{aligned}
& \left|\left[m(\tau)-p(\tau)^{-1} \bar{m}_{p}(\tau)\right]^{2}-h^{4} B_{a s}(\tau)^{2}\right| \\
& \quad \leq C \max \left\{h^{3},(n h)^{-1}\right\}\left[C \max \left\{h^{3},(n h)^{-1}\right\}+2 h^{2} B_{a s}(\tau)\right] \\
& \quad \leq C_{1} \max \left\{h^{5}, n^{-1} h,(n h)^{-2}\right\},
\end{aligned}
$$

and therefore, with $\sigma_{D}^{2}(\tau)=h^{4} B_{a s}(\tau)^{2} \Omega_{D}(\tau) \kappa_{2}$,

$$
\sup _{\tau \in[\delta, 1-\delta]}\left|\mathbb{E} Z_{n, D}(\tau)^{2}-\sigma_{D}^{2}(\tau)\right| \leq C_{1} \max \left\{h^{5}, n^{-1} h,(n h)^{-2}\right\}+C_{2} \phi_{n}
$$

It then follows directly that

$$
\begin{aligned}
\sup _{\tau \in[\delta, 1-\delta]}\left\|I I_{n, 1}(\tau)\right\| & \leq \frac{1}{\epsilon^{*}}\left(\sup _{\tau \in[\delta, 1-\delta]}\left|\mathbb{E} Z_{n, D}(\tau)^{2}-\sigma_{D}^{2}(\tau)\right|^{1 / 2}+\sup _{\tau \in[\delta, 1-\delta]}\left|\sigma_{D}(\tau)\right|\right) \\
& \leq C \max \left\{h^{2}, \sqrt{\frac{h}{n}}, \frac{1}{n h}\right\} .
\end{aligned}
$$

As $\sup _{\tau \in[\delta, 1-\delta]}\|\hat{p}(\tau)-p(\tau)\| \leq C \max \left\{h^{2}, \frac{1}{\sqrt{n h}}\right\}$, it follows that

$$
\sup _{\tau \in[\delta, 1-\delta]}\left\|I I_{n, 2}(\tau)\right\| \leq C \max \left\{\sqrt{n h^{9}}, \frac{1}{\sqrt{n h}}\right\} .
$$

Furthermore, by Lemma 2.4(i),

$$
\left\|\bar{m}_{D}(\tau)-\bar{m}_{p}(\tau)\right\| \leq \frac{1}{\sqrt{n h}}\left\|Z_{n, D, m, 0}(\tau)\right\| \leq \frac{C}{\sqrt{n h}},
$$


such that it also follows that

$$
\begin{aligned}
\sup _{\tau \in[\delta, 1-\delta]}\left\|I I_{n, 3}(\tau)\right\| & \leq C \sup _{\tau \in[\delta, 1-\delta]}\|\hat{p}(\tau)-p(\tau)\|\left\|\bar{m}_{\mid D}(\tau)-\bar{m}_{p}(\tau)\right\| \\
& \leq C \max \left\{h^{2}, \frac{1}{\sqrt{n h}}\right\}
\end{aligned}
$$

Finally, it follows directly from Lemma $2.3(i)$ that

$$
\sup _{\tau \in[\delta, 1-\delta]}\left|I I I_{n}(\tau)\right| \leq C \max \left\{\sqrt{n h^{7}}, \frac{1}{\sqrt{n h}}\right\} .
$$

Collecting all remainder terms in $R_{n}(\tau)=I_{n, 1}(\tau)+I I_{n, 2}(\tau)+I I_{n, 3}(\tau)+I I I_{n}(\tau)$, we find that $\sup _{\tau \in[\delta, 1-\delta]}\left\|R_{n}(\tau)\right\| \leq C \max \left\{h^{2}, \sqrt{n h^{7}}, \frac{1}{\sqrt{n h}}\right\}$.

Proof of Theorem 2.1. Given Lemma 2.6, we only have to prove asymptotic normality of $Z_{n, U}(\tau)=\frac{1}{\sqrt{n h}} \sum_{t=1}^{n} k_{t}(\tau) D_{t} \sigma_{t} u_{t}$. To simplify the proofs, we first condition on $\left\{D_{t}\right\}_{t=1}^{n}$ and thus prove conditional asymptotic normality of $Z_{n, U}(\tau)=$ $\frac{1}{\sqrt{n h}} \sum_{t=1}^{n} k_{t}(\tau) D_{t} \sigma_{t} u_{t}$. As before, let $\mathbb{E}_{D}(\cdot)=\mathbb{E}\left(\cdot \mid\left\{D_{t}\right\}_{t=1}^{\infty}\right)$. As the limit results do not depend on $\left\{D_{t}\right\}_{t=1}^{n}$, the results then directly hold unconditionally as well.

Take an $M$ such that $M \rightarrow \infty$ as $n \rightarrow \infty$, and truncate the $\mathrm{MA}(\infty)$ representation of $u_{t}$ at $M$ lags, say $u_{t, M}=\sum_{j=1}^{M} \psi_{j} \epsilon_{t-j}$. Then we can write

$$
\begin{aligned}
Z_{n}(\tau) & =\frac{1}{\sqrt{n h}} \sum_{t=1}^{n} k_{t}(\tau) D_{t} \sigma_{t} u_{t, M}+\frac{1}{\sqrt{n h}} \sum_{t=1}^{n} k_{t}(\tau) D_{t} \sigma_{t} \sum_{j=M+1}^{\infty} \psi_{j} \epsilon_{t-j} \\
& =\bar{Z}_{n, M}(\tau)+\bar{W}_{n, M}(\tau) .
\end{aligned}
$$

Let $R_{W}(k)=\mathbb{E}\left(\sum_{j=M+1}^{\infty} \psi_{j} \epsilon_{t-j}\right)\left(\sum_{j=M+1}^{\infty} \psi_{j} \epsilon_{t+k-j}\right)=\sigma_{\varepsilon}^{2} \sum_{j=M+1}^{\infty} \psi_{j} \psi_{j+|k|}$. Then

$$
\begin{aligned}
\mathbb{E}_{D} \bar{W}_{n, M}^{2} & =(n h)^{-1} \sum_{t=1}^{n} \sum_{s=1}^{n} D_{t} D_{s} \sigma_{t} \sigma_{s} k_{t}(\tau) k_{s}(\tau) R_{W}(t-s) \\
& \leq 2(n h)^{-1} \sup _{\tau \in[0,1]} \sigma(\tau)^{2} \sum_{i=0}^{n} \sum_{t=1}^{n-i} k_{t}(\tau) k_{t+i}(\tau)\left|R_{W}(i)\right| \\
& \leq 2 C \sup _{\tau \in[0,1]} \sigma(\tau)^{2} \sum_{i=0}^{\infty}\left|\sum_{j=M+1}^{\infty} \psi_{j} \psi_{j+i}\right|
\end{aligned}
$$




$$
\leq 2 C \sup _{\tau \in[0,1]} \sigma(\tau)^{2}\left(\sum_{j=M+1}^{\infty}\left|\psi_{j}\right|\right)^{2}=o\left(M^{-2}\right)
$$

which follows as $0 \leq D_{t} D_{t+i} \leq 1$, by Lemma 2.2 and the fact that the summability condition in Assumption 2.3 implies that $\sum_{j=M+1}^{\infty}\left|\psi_{j}\right|=o\left(M^{-1}\right)$. As $\mathbb{E}_{D} \bar{W}_{n, M}=$ 0 , the Markov inequality implies that the truncation is asymptotically negligible.

We next split $\bar{Z}_{n, M}(\tau)$ into two sequences of blocks: one with small, negligible blocks $Y_{n, j}(\tau)$ and one with dominating blocks $X_{n, j}(\tau)$. Define $B_{j}=(j-1)(a+b)$, then

$$
\begin{aligned}
& X_{n, j}(\tau)=\frac{1}{\sqrt{n h}} \sum_{t=B_{j}+1}^{B_{j}+a} k_{t}(\tau) D_{t} \sigma_{t} u_{t, M}, \\
& Y_{n, j}(\tau)=\frac{1}{\sqrt{n h}} \sum_{t=B_{j}+a+1}^{B_{j}+a+b} k_{t}(\tau) D_{t} \sigma_{t} u_{t, M},
\end{aligned}
$$

such that $\bar{Z}_{n, M}(\tau)=\sum_{j=1}^{k} X_{n, i}(\tau)+\sum_{i=1}^{k} Y_{n, i}(\tau)$, where $k=\lceil n /(a+b)\rceil$, and the final block is truncated to have $n$ observations in total. Now take sequences $a=a(n)$ and $b=b(n) \rightarrow \infty$ such that $a /(n h)+M / a \rightarrow 0$ and $b / a+M / b \rightarrow 0$ as $n \rightarrow \infty$.

We first show that the small blocks are asymptotically negligible. First note that $\mathbb{E}_{D}\left(\sum_{i=1}^{k} Y_{n, i}(\tau)\right)=0$. Consider $n$ large enough such that $a>M$ and the blocks $Y_{n, i}$ are mutually independent conditionally on $\left\{D_{t}\right\}_{t=1}^{n}$. Then, with $R_{M}(i)=\mathbb{E} u_{t, M} u_{t+i, M}$, where $\sum_{i=0}^{\infty}\left|R_{M}(i)\right|<\infty$ by Assumption 2.3, we have that

$$
\begin{aligned}
\mathbb{E}_{D}\left(\sum_{j=1}^{k} Y_{n, j}(\tau)\right)^{2} & =\sum_{j=1}^{k} \mathbb{E}_{D} Y_{n, j}(\tau)^{2} \\
& =\frac{1}{n h} \sum_{j=1}^{k} \sum_{s, t=B_{j}+a+1}^{B_{j}+a+b} k_{s}(\tau) k_{t}(\tau) D_{s} D_{t} \sigma_{s} \sigma_{t} R_{M}(s-t) \\
& \leq 2 C \frac{1}{n h} \sum_{i=0}^{b-1}\left|R_{M}(i)\right| \sum_{j=1}^{k} \sum_{t=B_{j}+a+1}^{B_{j}+a+b-i} k_{t}(\tau) k_{t+i}(\tau) \\
& \leq C_{1} \frac{1}{n h} \max _{0 \leq i \leq b-1} \sum_{j=1}^{k} \sum_{t=B_{i}+a+1}^{B_{i}+a+b-i} k_{t}(\tau) k_{t+i}(\tau)
\end{aligned}
$$




$$
\leq C_{2} \frac{k b h}{n h} \leq C_{3} \frac{b}{a}=o(1)
$$

where we use that $\sum_{j=1}^{k} \sum_{t=B_{i}+a+1}^{B_{i}+a+b-i} k_{t}(\tau) k_{t+i}(\tau) \leq C k b h$ and $k \sim a n$.

We next employ the Lindeberg central limit theorem (see e.g. Davidson, 2002, Thm 23.6) to show that $\sum_{j=1}^{k} X_{n, j}(\tau) \stackrel{d}{\rightarrow} \mathcal{N}\left(0, p(\tau)^{2} \sigma_{a s}^{2}(\tau)\right)$. Consider again $n$ sufficiently large such that such that $b>M$ and the blocks $X_{n, i}(\tau)$ are conditionally independent. Then $\mathbb{E}_{D} \sum_{j=1}^{k} X_{n, j}(\tau)=0$ and

$$
\begin{aligned}
\mathbb{E}_{D}\left(\sum_{j=1}^{k} X_{n, j}(\tau)\right)^{2}= & \sum_{j=1}^{k} \mathbb{E}_{D} X_{n, j}(\tau)^{2} \\
= & \frac{1}{n h} \sum_{i=1}^{k} \sum_{s, t=B_{j}+1}^{B_{j}+a} k_{s}(\tau) k_{t}(\tau) D_{s} D_{t} \sigma_{s} \sigma_{t} R_{M}(s-t) \\
= & \frac{1}{n h} \sum_{j=1}^{k} \sum_{i=-a+1}^{a-1} R_{M}(i) \sum_{t=B_{j}+1}^{B_{j}+a-|i|} k_{t}(\tau) k_{t+|i|}(\tau) \sigma_{t} \sigma_{t+|i|} \\
& \times D_{t} D_{t+|i|} .
\end{aligned}
$$

As $M \rightarrow \infty, R_{M}(k) \rightarrow R_{U}(k)$. A straightforward extension of Lemma 2.4 then shows that $\mathbb{E}_{D}\left(\sum_{j=1}^{k} X_{n, j}(\tau)\right)^{2} \stackrel{p}{\rightarrow} p(\tau) \sigma(\tau)^{2} \Omega \kappa_{2}$.

The final step is to verify the Lindeberg condition, that is, we verify that, for every $\kappa>0, \sum_{j=1}^{k} \mathbb{E}_{D}\left[\frac{X_{n, j}(\tau)^{2}}{\omega_{n}^{2}} \mathbb{1}\left(\left|\frac{X_{n, i}(\tau)}{\omega_{n}}\right|>\kappa\right)\right]=o_{p}(1)$, with $\omega_{n}^{2}=\mathbb{E}_{D}\left(\sum_{j=1}^{k} X_{n, j}(\tau)\right)^{2}$. Note that

$$
\sum_{j=1}^{k} \mathbb{E}_{D}\left[\frac{X_{n, j}(\tau)^{2}}{\omega_{n}^{2}} \mathbb{1}\left(\left|\frac{X_{n, i}(\tau)}{\omega_{n}}\right|>\kappa\right)\right] \leq \frac{1}{\kappa^{2} \omega_{n}^{4}} \sum_{j=1}^{k} \mathbb{E}_{D} X_{n, j}(\tau)^{4}
$$

and define $\tilde{X}_{t}(\tau)=D_{t} k_{t}(\tau) \sigma_{t} u_{t, M}$, which, due to the $M$-dependence of $u_{t, M}$, is an $L_{4}$-mixingale with $\mathbb{E}_{D} \tilde{X}_{t}^{4} \leq C k_{t}(\tau)^{4} \mathbb{E} u_{t, M}^{4}$, where it follows from Minkowski's inequality that

$$
\mathbb{E} u_{t, M}^{4} \leq\left(\sum_{j=0}^{M}\left(\mathbb{E}\left|\psi_{j} \varepsilon_{t-j}\right|^{4}\right)^{1 / 4}\right)^{4} \leq C \mathbb{E} \varepsilon_{t}^{4}\left(\sum_{j=0}^{M} \psi_{j}\right)^{4}<\infty
$$


by stationarity of $\left\{\varepsilon_{t}\right\}$ and $\mathbb{E} \varepsilon_{t}^{4}<\infty$. Lemma 2 of Hansen (1991) then implies that

$$
\mathbb{E}_{D} X_{n, j}(\tau)^{4}=\mathbb{E}_{D}\left|\frac{1}{\sqrt{n h}} \sum_{t=B_{j}+1}^{B_{j}+a} \tilde{X}_{t}(\tau)\right|^{4} \leq C \frac{1}{(n h)^{2}}\left(\sum_{t=B_{j}+1}^{B_{j}+a} k_{t}(\tau)^{2}\right)^{2}
$$

As the blocks are non-overlapping, it follows from the properties of the kernel function and the $c_{r}$-inequality that

$$
\sum_{j=1}^{k}\left(\sum_{t=B_{j}+1}^{B_{j}+a} k_{t}(\tau)^{2}\right)^{2} \leq a \sum_{j=1}^{k} \sum_{t=B_{j}+1}^{B_{j}+a} k_{t}(\tau)^{4} \leq \text { Cnha. }
$$

Therefore, with $\omega_{n}^{-2}=O(1)$ and $a=o(n h)$,

$$
\sum_{i=1}^{k} \mathbb{E}_{D}\left[\frac{X_{n, i}(\tau)^{2}}{\omega_{n}^{2}} \mathbb{1}\left(\left|\frac{X_{n, i}(\tau)}{\omega_{n}}\right|>\kappa\right)\right] \leq \frac{C a}{\omega_{n}^{4} n h}=o(1)
$$

Lemma 2.7. Let Assumptions 2.1-2.8 hold. Then, for any $0<\delta<\delta^{*}<\frac{1}{2}$ and some $N>0$, it holds for all $\tau \in\left[\delta^{*}, 1-\delta^{*}\right]$ and $n>N$ that

$$
\sqrt{n h}\left[\hat{m}^{*}(\tau)-\tilde{m}(\tau)-h^{2} B_{a s}(\tau)\right]=p(\tau)^{-1} Z_{n, U}^{*}(\tau)+R_{n}^{*}(\tau),
$$

where

$$
\begin{gathered}
Z_{n, U}^{*}(\tau)=\frac{1}{\sqrt{n h}} \sum_{t=1}^{n} k_{t}(\tau) D_{t} z_{t} \xi_{t}^{*} \\
\operatorname{plim}_{n \rightarrow \infty} \operatorname{Var}^{*} Z_{n, U}^{*}(\tau)=\sigma_{Z}^{2}(\tau) \\
\sup _{\tau \in\left[\delta^{*}, 1-\delta^{*}\right]} \mathbb{E}\left\|R_{n}^{*}(\tau)\right\|^{*} \leq C \max \left\{\frac{1}{\sqrt{n h}}, \sqrt{n h^{7}}, \sqrt{h \tilde{h}^{-1}}, \sqrt{n h^{5} \tilde{h}^{4}},\right. \\
\left.\tilde{h}^{2}, \sqrt{\ell \tilde{h}^{4}}, \sqrt{\frac{\ell}{n \tilde{h}}}\right\},
\end{gathered}
$$

and $B_{a s}(\tau)$ and $\sigma_{Z}^{2}(\tau)$ are defined in (2.8) and (2.17b) respectively. 
Proof of Lemma 2.7. Analogously to the proof of Lemma 2.6, we define

$$
\begin{array}{ll}
\breve{m}^{*}(\tau)=\frac{1}{n h} \sum_{t=1}^{n} k_{t}(\tau) D_{t} y_{t}^{*}, & \bar{m}_{D}^{*}(\tau)=\frac{1}{n h} \sum_{t=1}^{n} k_{t}(\tau) D_{t} \tilde{m}\left(\frac{t}{n}\right) \\
\bar{m}_{p}^{*}(\tau)=\frac{1}{n h} \sum_{t=1}^{n} p\left(\frac{t}{n}\right) k_{t}(\tau) \tilde{m}\left(\frac{t}{n}\right), &
\end{array}
$$

such that we can write

$$
\begin{aligned}
& \sqrt{n h}\left[\hat{m}^{*}(\tau)-\tilde{m}(\tau)-h^{2} B_{a s}(\tau)\right]=\sqrt{n h} \hat{p}(\tau)^{-1}\left\{\breve{m}^{*}(\tau)-\bar{m}_{D}^{*}(\tau)\right\} \\
& \quad+\left\{\hat{p}(\tau)^{-1} \bar{m}_{D}^{*}(\tau)-p(\tau)^{-1} \bar{m}_{p}^{*}(\tau)\right\}+\sqrt{n h}\left\{p(\tau)^{-1} \bar{m}_{p}^{*}(\tau)-\tilde{m}(\tau)-h^{2} B_{a s}(\tau)\right\} \\
& =p(\tau)^{-1} Z_{n, U}^{*}(\tau)+I_{n}^{*}(\tau)+I I_{n}^{*}(\tau)+I I I_{n}^{*}(\tau)
\end{aligned}
$$

As a general observation, note that taking the sums in the expressions above from $t=1$ to $n$ appears to include the boundary points for $\tilde{m}(\cdot)$, for which the properties of the estimator are not satisfied. However, as we consider $\tau \in\left[\delta^{*}, 1-\delta^{*}\right]$ and $\tilde{m}\left(\frac{t}{n}\right)$ is always multiplied by $k_{t}(\tau), k_{t}(\tau)=0$ at all points $\frac{t}{n}<\delta$ and $\frac{t}{n}>1-\delta$ for large enough $n$, and therefore

$$
\frac{1}{n h} \sum_{t=1}^{n} k_{t}(\tau) \tilde{m}\left(\frac{t}{n}\right)=\frac{1}{n h} \sum_{t=[n \delta]+1}^{[n(1-\delta)]} k_{t}(\tau) \tilde{m}\left(\frac{t}{n}\right)
$$

and analogously for all related sums. Hence, in the following we simply take sums from $t=1$ to $n$, under the implicit assumption that $n$ is large enough to do so.

We now first derive (2.21b). With

$$
\mathbb{E}^{*} Z_{n, U}^{*}(\tau)^{2}=\frac{1}{n h} \sum_{i=-n+1}^{n-1} \sum_{t=1}^{n-|i|} k_{t}(\tau) k_{t+|i|}(\tau) D_{t} D_{t+|i|} z_{t} z_{t+|i|} \gamma^{|i|}
$$

it follows from Lemma 2.5 and the fact that $\sum_{i=0}^{\infty} \gamma^{i}=\frac{1}{1-\theta^{1 / \ell}}=-\ell / \ln \theta+o(\ell)$,

$$
\mathbb{E}\left|\mathbb{E}^{*} Z_{n, U}^{*}(\tau)^{2}-\mathbb{E}_{D} \mathbb{E}^{*} Z_{n, U}^{*}(\tau)^{2}\right| \leq \phi_{n} \sum_{i=0}^{\infty} \gamma^{i} \beta_{i}+\frac{\eta_{n}}{\sqrt{n h}} \sum_{i=0}^{\infty} \gamma^{i} \leq \frac{C \ell}{\sqrt{n h}}=o(1)
$$


Furthermore

$$
\begin{aligned}
\mathbb{E}_{D} \mathbb{E}^{*} Z_{n, U}^{*}(\tau)^{2}= & \frac{1}{n h} \sum_{i=-n+1}^{n-1} R_{U}(i) \sum_{t=1}^{n-|i|} k_{t}(\tau) k_{t+|i|}(\tau) D_{t} D_{t+|i|} \sigma_{t} \sigma_{t+|i|} \gamma^{|i|} \\
= & \mathbb{E}_{D} Z_{n, U}^{2}+\frac{1}{n h} \sum_{i=-n+1}^{n-1} R_{U}(i) \sum_{t=1}^{n-|i|} k_{t}(\tau) k_{t+|i|}(\tau) \sigma_{t} \sigma_{t+|i|} \\
& \times D_{t} D_{t+|i|}\left(\gamma^{|i|}-1\right)
\end{aligned}
$$

where $Z_{n, U}$ is defined in Lemma 2.6. For the second term, take $M=M(n)$ such that $1 / M+M^{2} / \ell \rightarrow 0$ as $n \rightarrow \infty$, then we have that

$$
\begin{aligned}
& \sup _{\tau \in\left[\delta^{*}, 1-\delta^{*}\right]}\left|\frac{1}{n h} \sum_{i=-n+1}^{n-1} R_{U}(i)\left(\gamma^{|i|}-1\right) \sum_{t=1}^{n-|i|} k_{t}(\tau) k_{t+|i|}(\tau) \sigma_{t} \sigma_{t+|i|} D_{t} D_{t+|i|}\right| \\
& \leq C \sum_{i=1}^{M} R_{U}(i)\left|\theta^{i / \ell}-1\right|+C \sum_{i=M+1}^{n-1} R_{U}(i)\left|\theta^{i / \ell}-1\right| \\
& \leq C_{1} \sum_{i=1}^{M}\left(1-\theta^{i / \ell}\right)+C_{2} \sum_{i=M+1}^{\infty} R_{U}(i)=o(1),
\end{aligned}
$$

as $\sum_{i=1}^{M}\left(1-\theta^{i / \ell}\right) \leq C \sum_{i=1}^{M}(-\ln \theta) i / \ell \leq C_{1} M^{2} / \ell=o(1)$ and $\sum_{i=M+1}^{\infty} R_{U}(i)=$ $o\left(M^{-1}\right)$. It then follows directly from the proof of Lemma 2.6 that $\operatorname{plim}_{n \rightarrow \infty} \operatorname{Var}^{*} Z_{n, U}^{*}(\tau)=\sigma_{Z}^{2}(\tau)$ for all $\tau \in\left[\delta^{*}, 1-\delta^{*}\right]$.

Now consider $I_{n}^{*}(\tau)$, which we write as

$$
\begin{aligned}
I_{n}^{*}(\tau) & =\left[\hat{p}(\tau)^{-1}-p(\tau)^{-1}\right] Z_{n, U}^{*}(\tau)+\frac{1}{\sqrt{n h}} \sum_{t=1}^{n} k_{t}(\tau)\left[m\left(\frac{t}{n}\right)-\tilde{m}\left(\frac{t}{n}\right)\right] D_{t} \xi_{t}^{*} \\
& =I_{n, 1}^{*}(\tau)+I_{n, 2}^{*}(\tau) .
\end{aligned}
$$

It follows directly as in the proof of Lemma 2.6 and using Jensen's inequality that $\sup _{\tau \in\left[\delta^{*}, 1-\delta^{*}\right]} \mathbb{E}\left\|I_{n, 1}^{*}(\tau)\right\|^{*} \leq C \max \left\{h^{2}, \frac{1}{\sqrt{n h}}\right\}$. Furthermore, as

$$
\begin{aligned}
\mathbb{E}^{*} I_{n, 2}^{*}(\tau)^{2}= & \frac{1}{(n h)^{2}} \sum_{s=1}^{n} \sum_{t=1}^{n} k_{s}(\tau) k_{t}(\tau)\left[\tilde{m}\left(\frac{s}{n}\right)-m\left(\frac{t}{n}\right)\right]\left[\tilde{m}\left(\frac{t}{n}\right)-m\left(\frac{t}{n}\right)\right] \\
& \times \gamma^{|s-t|} D_{s} D_{t},
\end{aligned}
$$


it follows from Jensen's and the Cauchy-Schwartz inequality that

$$
\begin{aligned}
\mathbb{E}\left\|I_{n, 2}^{*}(\tau)\right\|^{*} \leq & {\left[\frac{2}{n h} \sum_{i=0}^{n-1} \gamma^{i} \sum_{t=1}^{n-i} k_{t}(\tau) k_{t+i}(\tau)\left\|\tilde{m}\left(\frac{t}{n}\right)-m\left(\frac{t}{n}\right)\right\|\right.} \\
& \left.\times\left\|\tilde{m}\left(\frac{t+i}{n}\right)-m\left(\frac{t+i}{n}\right)\right\|\right]^{1 / 2} \\
\leq & C\left[\sup _{\sup _{\tau \in[\delta, 1-\delta]}}\|\tilde{m}(\tau)-m(\tau)\|^{2} \sum_{i=0}^{\infty} \gamma^{i}\right]^{1 / 2} \\
\leq & C_{1} \sqrt{\ell} \max \left\{\tilde{h}^{2}, \frac{1}{\sqrt{n \tilde{h}}}\right\},
\end{aligned}
$$

as, using Lemma 2.6,

$$
\begin{aligned}
\sup _{\tau \in[\delta, 1-\delta]}\|\tilde{m}(\tau)-m(\tau)\| \leq & \tilde{h}^{2} \sup _{\tau \in[\delta, 1-\delta]}\left|B_{a s}(\tau)\right|+\frac{1}{\sqrt{n \tilde{h}}} \sup _{\tau \in[\delta, 1-\delta]}\left\|Z_{n, U}(\tau)\right\| \\
& +\frac{1}{\sqrt{n \tilde{h}}} \sup _{\tau \in[\delta, 1-\delta]}\left\|R_{n}(\tau)\right\| \leq C \max \left\{\tilde{h}^{2}, \frac{1}{\sqrt{n \tilde{h}}}\right\} .
\end{aligned}
$$

As in the proof of Lemma 2.6, write $I I_{n}^{*}(\tau)$ as

$$
\begin{aligned}
I I_{n}^{*}(\tau)= & p(\tau)^{-1}\left\{\bar{m}_{D}^{*}(\tau)-p(\tau)^{-1} \hat{p}(\tau) \bar{m}_{p}^{*}(\tau)\right\} \\
& +\hat{p}(\tau)^{-1} p(\tau)^{-2}\{\hat{p}(\tau)-p(\tau)\}^{2} \bar{m}_{p}^{*}(\tau) \\
& +\left\{\hat{p}(\tau)^{-1}-p(\tau)^{-1}\right\}\left\{\bar{m}_{D}^{*}(\tau)-\bar{m}_{p}^{*}(\tau)\right\} \\
= & I I_{n, 1}^{*}(\tau)+I I_{n, 2}^{*}(\tau)+I I_{n, 3}^{*}(\tau),
\end{aligned}
$$

where $I I_{n, 1}^{*}(\tau)=p(\tau)^{-1} Z_{n, D}^{*}(\tau)$ and

$$
Z_{n, D}^{*}(\tau)=\frac{1}{\sqrt{n h}} \sum_{t=1}^{n} k_{t}(\tau)\left[\tilde{m}\left(\frac{t}{n}\right)-p(\tau)^{-1} \bar{m}_{p}^{*}(\tau)\right]\left[D_{t}-p\left(\frac{t}{n}\right)\right]
$$


Define $Z_{n, D}(\tau)=\frac{1}{\sqrt{n h}} \sum_{t=1}^{n} k_{t}(\tau)\left[m\left(\frac{t}{n}\right)-p(\tau)^{-1} \bar{m}_{p}(\tau)\right]\left[D_{t}-p\left(\frac{t}{n}\right)\right]$. Then

$$
\begin{aligned}
Z_{n, D}^{*}(\tau)-Z_{n, D}(\tau)= & \frac{1}{\sqrt{n h}} \sum_{t=1}^{n} k_{t}(\tau)\left[\tilde{m}\left(\frac{t}{n}\right)-m\left(\frac{t}{n}\right)\right]\left[D_{t}-p\left(\frac{t}{n}\right)\right] \\
& +\frac{1}{\sqrt{n h}} p(\tau)^{-1}\left[\bar{m}_{p}^{*}(\tau)-\bar{m}_{p}(\tau)\right] \sum_{t=1}^{n} k_{t}(\tau)\left[D_{t}-p\left(\frac{t}{n}\right)\right] \\
= & I I_{n, 11}^{*}(\tau)+I I_{n, 12}^{*}(\tau) .
\end{aligned}
$$

It follows directly by $(2.23)$ that

$$
\begin{aligned}
\left\|I I_{n, 11}^{*}(\tau)\right\| \leq & \left(\sup _{\tau \in\left[\delta^{*}, 1-\delta^{*}\right]}\|\tilde{m}(\tau)-m(\tau)\|^{2}\right. \\
& \left.\times \sum_{i=-n+1}^{n-1} \frac{1}{n h} \sum_{t=1}^{n-|i|} k_{t}(\tau) k_{t+|i|}(\tau)\left|\operatorname{Cov}\left(D_{t}, D_{t+|i|}\right)\right|\right)^{1 / 2} \\
\leq & C \max \left\{\tilde{h}^{2}, \frac{1}{\sqrt{n \tilde{h}}}\right\} .
\end{aligned}
$$

Furthermore, as

$$
\begin{aligned}
& \sup _{\tau \in\left[\delta^{*}, 1-\delta^{*}\right]}\left\|\bar{m}_{p}^{*}(\tau)-\bar{m}_{p}(\tau)\right\| \\
& \quad \leq \sup _{\tau \in\left[\delta^{*}, 1-\delta^{*}\right]}\left\|\tilde{m}_{n}(\tau)-m(\tau)\right\| \sup _{\tau \in\left[\delta^{*}, 1-\delta^{*}\right]} \frac{1}{n h} \sum_{t=1}^{n} p\left(\frac{t}{n}\right) k_{t}(\tau) \\
& \quad \leq C \max \left\{\tilde{h}^{2}, \frac{1}{\sqrt{n \tilde{h}}}\right\}
\end{aligned}
$$

and $\frac{1}{\sqrt{n h}}\left\|\sum_{t=1}^{n} k_{t}(\tau)\left[D_{t}-p\left(\frac{t}{n}\right)\right]\right\| \leq C_{1}$ by Lemma $2.4(i)$, it follows that

$$
\sup _{\tau \in\left[\delta^{*}, 1-\delta^{*}\right]}\left\|I I_{n, 12}^{*}(\tau)\right\| \leq C \max \left\{\tilde{h}^{2}, \frac{1}{\sqrt{n \tilde{h}}}\right\} .
$$

As it was shown in Lemma 2.6 that $\left\|Z_{n, D}(\tau)\right\| \leq C h^{2}$, it follows that $\left\|I I_{n, 1}^{*}(\tau)\right\| \leq$ $C \max \left\{\tilde{h}^{2}, \frac{1}{\sqrt{n \tilde{h}}}\right\}$. 
As $\sup _{\tau \in[\delta, 1-\delta]}\left\|\bar{m}_{p}^{*}(\tau)-\bar{m}_{p}(\tau)\right\| \leq C \max \left\{\tilde{h}^{2}, \frac{1}{\sqrt{n \tilde{h}}}\right\}$, it follows as in the proof of $I I_{n, 2}(\tau)$ in Lemma 2.6 that

$$
\sup _{\tau \in\left[\delta^{*}, 1-\delta^{*}\right]}\left\|I I_{n, 2}^{*}(\tau)\right\| \leq C \max \left\{\sqrt{n h^{9}}, \frac{1}{\sqrt{n h}}\right\},
$$

while $\bar{m}_{D}^{*}(\tau)-\bar{m}_{p}^{*}(\tau)=\bar{m}_{D}(\tau)-\bar{m}_{p}(\tau)+I I_{n, 11}^{*}(\tau)$, and $I I_{n, 11}^{*}(\tau)$ is defined in (2.24). Therefore it follows directly as in the proof of $I I_{n, 3}(\tau)$ in Lemma 2.6 that

$$
\sup _{\tau \in[\delta, 1-\delta]}\left\|I I_{n, 3}^{*}(\tau)\right\| \leq C \max \left\{\tilde{h}^{2}, \frac{1}{\sqrt{n h}}\right\} .
$$

It follows from Lemma 2.6 that $\tilde{m}(\tau)=m(\tau)+\tilde{h}^{2} B_{a s}+\tilde{R}_{n}(\tau)$, where $\sup _{\tau \in[\delta, 1-\delta]}\left\|\tilde{R}_{n}(\tau)\right\| \leq \frac{C}{\sqrt{n \tilde{h}}}$. Substituting this into $I I I_{n}^{*}(\tau)$ we find that

$$
\begin{aligned}
I I I_{n}^{*}(\tau)= & {\left[p(\tau)^{-1} \frac{1}{\sqrt{n h}} \sum_{t=1}^{n} p\left(\frac{t}{n}\right) k_{t}(\tau) m\left(\frac{t}{n}\right)-m(\tau)-h^{2} B_{a s}(\tau)\right] } \\
& +\tilde{h}^{2}\left[p(\tau)^{-1} \frac{1}{\sqrt{n h}} \sum_{t=1}^{n} p\left(\frac{t}{n}\right) k_{t}(\tau) B_{a s}\left(\frac{t}{n}\right)-B_{a s}(\tau)\right] \\
& +\left[p(\tau)^{-1} \frac{1}{\sqrt{n h}} \sum_{t=1}^{n} p\left(\frac{t}{n}\right) k_{t}(\tau) \tilde{R}_{n}\left(\frac{t}{n}\right)-\tilde{R}_{n}(\tau)\right] \\
= & I I I_{n, 1}^{*}(\tau)+I I I_{n, 2}^{*}(\tau)+I I I_{n, 3}^{*}(\tau) .
\end{aligned}
$$

Note that $I I I_{n, 1}^{*}(\tau)$ is equal to $I I I_{n}(\tau)$ defined in Lemma 2.6, such that $\sup _{\tau \in\left[\delta^{*}, 1-\delta^{*}\right]}\left|I I I_{n, 1}^{*}(\tau)\right| \leq C \max \left\{\sqrt{n h^{7}}, \frac{1}{\sqrt{n h}}\right\}$. Furthermore, using the definition of $B_{a s}(\tau)$ in $(2.8)$, we can write

$$
\frac{1}{\sqrt{n h}} \sum_{t=1}^{n} p\left(\frac{t}{n}\right) k_{t}(\tau) B_{a s}\left(\frac{t}{n}\right)=\frac{1}{2} \mu_{2} \frac{1}{\sqrt{n h}} \sum_{t=1}^{n} k_{t}(\tau) f\left(\frac{t}{n}\right),
$$

where $f(\tau)=p(\tau)^{-1}[m p]^{(2)}(\tau)$ is Lipschitz continuous. Then, by Lemma $2.3(i)$ it follows that

$$
\begin{aligned}
\left|I I I_{n, 2}^{*}(\tau)\right| & \leq C \sqrt{n h \tilde{h}^{2}}\left|\frac{1}{n h} \sum_{t=1}^{n} k_{t}(\tau) f\left(\frac{t}{n}\right)-f(\tau)\right| \\
& \leq C_{1} \max \left\{\frac{\tilde{h}^{2}}{\sqrt{n h}}, \sqrt{n h^{5} \tilde{h}^{4}}\right\} .
\end{aligned}
$$


Finally, for $I I I_{n, 3}^{*}(\tau)$ we have

$$
\begin{aligned}
\left\|I I I_{n}^{*}(\tau)\right\| & \leq C \sqrt{n h} \sup _{\tau \in[\delta, 1-\delta]}\left\|\tilde{R}_{n}(\tau)\right\| \frac{1}{n h} \sum_{t=1}^{n} k_{t}^{2}(\tau)+\left\|\tilde{R}_{n}(\tau)\right\| \\
& \leq C_{1} \sqrt{\frac{h}{\tilde{h}}}+\frac{C_{2}}{\sqrt{n \tilde{h}}} .
\end{aligned}
$$

Collecting all remainder terms, it then follows that

$\sup _{\tau \in\left[\delta^{*}, 1-\delta^{*}\right]} \mathbb{E}\left\|R_{n}^{*}(\tau)\right\|^{*} \leq C \max \left\{\frac{1}{\sqrt{n h}}, \sqrt{n h^{7}}, \sqrt{\frac{h}{\tilde{h}}}, \sqrt{n h^{5} \tilde{h}^{4}}, \tilde{h}^{2}, \sqrt{\ell \tilde{h}^{4}}, \sqrt{\frac{\ell}{n \tilde{h}}}\right\} . \square$

Proof of Theorem 2.2. Given Lemma 2.7, we only have to prove asymptotic normality of $Z_{n, U}^{*}(\tau)$. As in the proof of Theorem 2.1, we establish asymptotic normality of the bootstrap process using a blocking technique. By the stationarity of $\xi_{t}^{*}$, we can write $\xi_{t}^{*}=\sum_{j=0}^{\infty} \gamma^{j} \nu_{t-j}^{*}$ with $\nu_{t}^{*}$ for $t \leq 1$ defined analogously as for $t>1$. Take an $M$ such that $M / \ell \rightarrow \infty$ as $n \rightarrow \infty$, and truncate the $\operatorname{MA}(\infty)$ representation of $\xi_{t}^{*}$ at $M$ lags to define $\xi_{t, M}^{*}=\sum_{j=0}^{M} \gamma^{j} \nu_{t-j}^{*}$. Then we write

$$
\begin{aligned}
Z_{n, U}^{*}(\tau) & =\frac{1}{\sqrt{n h}} \sum_{t=1}^{n} k_{t}(\tau) D_{t} z_{t} \xi_{t, M}^{*}+\frac{1}{\sqrt{n h}} \sum_{t=1}^{n} k_{t}(\tau) D_{t} z_{t}\left(\sum_{j=M+1}^{\infty} \gamma^{j} \nu_{t-j}^{*}\right) \\
& =\bar{Z}_{n, M}^{*}(\tau)+\bar{W}_{n, M}^{*}(\tau) .
\end{aligned}
$$

Applying Markov's inequality twice, we have that

$$
\begin{gathered}
\mathbb{E} \mathbb{E}^{*} \bar{W}_{n, M}^{*}(\tau)^{2} \leq \frac{2}{n h}\left(1-\gamma^{2}\right) \sum_{i=0}^{n-1}\left|R_{U}(i)\right| \sum_{t=1}^{n-i} k_{t}(\tau) k_{t+i}(\tau)\left(\sum_{j=M+1}^{\infty} \gamma^{2 j+i}\right) \\
\leq C \gamma^{2 M} \sum_{i=0}^{\infty} \gamma^{i}\left|R_{U}(i)\right|=C \theta^{2 M / \ell} \sum_{i=0}^{\infty} \theta^{i / \ell}\left|R_{U}(i)\right| \leq C_{1} \theta^{2 M / \ell}=o(1),
\end{gathered}
$$

as $M / \ell \rightarrow \infty$. It then follows that $\bar{W}_{n, M}^{*}(\tau)=o_{p}^{*}(1)$ for all $\tau \in\left[\delta^{*}, 1-\delta^{*}\right]$.

Now let $\bar{Z}_{t, M}(\tau)=\sum_{j=1}^{k} X_{n, j}^{*}(\tau)+\sum_{j=1}^{k} Y_{n, j}(\tau)$, where

$$
X_{n, j}^{*}(\tau)=\frac{1}{\sqrt{n h}} \sum_{t=B_{j}+1}^{B_{j}+a} k_{t}(\tau) D_{t} z_{t} \xi_{t, M}^{*}
$$




$$
Y_{n, j}(\tau)=\frac{1}{\sqrt{n h}} \sum_{t=B_{j}+a+1}^{B_{j}+a+b} k_{t}(\tau) D_{t} z_{t} \xi_{t, M}^{*}
$$

with $B_{j}=(j-1)(a+b)$ and $k=\lceil n /(a+b)\rceil$. Take sequences $a=a(n)$ and $b=b(n)$ such that $a /(n h)+M / a \rightarrow 0$ and $b / a+M / b \rightarrow 0$ as $n \rightarrow \infty$.

We first show that $\sum_{j=1}^{k} Y_{n, i}^{*}(\tau)=o_{p}^{*}(1)$. Consider $n$ large enough such that $a(n)>M$ and the blocks $Y_{n, i}$ are mutually independent conditionally on the original data. Then, with

$$
R_{M}^{*}(i)=\mathbb{E}^{*} \xi_{t}^{*} \xi_{t+|i|}^{*}=\left(1-\gamma^{2}\right) \sum_{j=0}^{M} \gamma^{j} \gamma^{j+|i|}=\theta^{|i| / \ell}\left(1-\theta^{2(M+1) / \ell}\right) \leq \theta^{|i| / \ell}
$$

for $|i| \leq M-1$, we have that

$$
\begin{aligned}
\mathbb{E} \mathbb{E}^{*}\left(\sum_{j=1}^{k} Y_{n, j}^{*}(\tau)\right)^{2} & =\sum_{j=1}^{k} \mathbb{E}^{*} Y_{n, j}(\tau)^{2} \\
& \leq \frac{1}{n h} \sum_{i=-b+1}^{b-1} \theta^{i / \ell} R_{U}(i) \sum_{j=1}^{k} \sum_{t=B_{j}+a+1}^{B_{j}+a+b-|i|} k_{t}(\tau) k_{t+|i|}(\tau) \\
& \leq C_{1} \frac{b}{a}=o(1) .
\end{aligned}
$$

As in the proof of Theorem 2.1, we employ the Lindeberg CLT to establish asymptotic normality of $\sum_{j=1}^{k} X_{n, j}^{*}(\tau)$, as for $n$ sufficiently large, $b>M$ and the blocks $X_{n, j}^{*}(\tau)$ are independent. First we show that the asymptotic variance is equal to $p(\tau)^{2} \sigma_{a s}^{2}(\tau)$. Note that $\mathbb{E}^{*} \sum_{j=1}^{k} X_{n, j}^{*}(\tau)=0$ and

$$
\begin{aligned}
& \mathbb{E}^{*}\left(\sum_{j=1}^{k} X_{n, j}^{*}(\tau)\right)^{2} \\
& =\frac{1}{n h} \sum_{i=-a+1}^{a-1} R_{M}^{*}(i) R_{U}(i) \sum_{j=1}^{k} \sum_{t=B_{j}+1}^{B_{j}+a-|i|} k_{t}(\tau) k_{t+|i|}(\tau) D_{t} D_{t+|i|} \sigma_{t} \sigma_{t+|i|} \\
& +\frac{1}{n h} \sum_{i=-a+1}^{a-1} \sum_{j=1}^{k} \sum_{t=B_{j}+1}^{B_{j}+a-|i|} k_{t}(\tau) k_{t+|i|}(\tau) D_{t} D_{t+|i|} \sigma_{t} \sigma_{t+|i|} R_{M}^{*}(i) \\
& \quad \times\left[u_{t} u_{t+|i|}-R_{U}(i)\right] \\
& =A_{X, n}^{*}(\tau)+B_{X, n}^{*}(\tau) .
\end{aligned}
$$


By adapting Lemma 2.5, we find that

$$
\begin{aligned}
\mathbb{E}\left|B_{X, n}^{*}(\tau)\right| \leq 2 \sum_{i=0}^{a-1} \mathbb{E} \mid \sum_{j=1}^{k} \sum_{t=B_{j}+1}^{B_{j}+a-|i|} k_{t}(\tau) k_{t+|i|}(\tau) D_{t} D_{t+|i|} \sigma_{t} \sigma_{t+|i|} \\
\quad\left[u_{t} u_{t+|i|}-R_{U}(i)\right] \mid \\
\leq \phi_{n} \sum_{i=0}^{a-1} \beta_{i}+\frac{\eta_{n}}{\sqrt{n h}}=o(1) .
\end{aligned}
$$

Furthermore, the arguments used to prove (2.21b) in Lemma 2.7 show that

$$
A_{X, n}^{*}(\tau) \stackrel{p}{\rightarrow} p(\tau)^{2} \sigma_{a s}^{2}(\tau)
$$

The final step is to verify that, for every $\kappa>0$,

$$
\sum_{j=1}^{k} \mathbb{E}^{*}\left[\frac{X_{n, j}^{*}(\tau)^{2}}{\omega_{n}^{* 2}} \mathbb{1}\left(\left|\frac{X_{n, j}^{*}(\tau)^{2}}{\omega_{n}^{* 2}}\right|>\kappa\right)\right]=o_{p}(1),
$$

where $\omega_{n}^{* 2}=\mathbb{E}^{*}\left(\sum_{j=1}^{k} X_{n, j}^{*}(\tau)\right)^{2}$. Similarly as in the proof of Theorem 2.1, we define $\tilde{X}_{t}^{*}(\tau)=D_{t} k_{t}(\tau) \sigma_{t} u_{t} \xi_{t, M}^{*}$, as an $L_{4}$-mixingale conditionally on the original data, with $\left\|\tilde{X}_{t}\right\|_{4}^{*} \leq C\left|u_{t}\right| k_{t}(\tau)$, such that Lemma 2 of Hansen (1991) then implies that $\mathbb{E}^{*} X_{n, i}(\tau)^{4} \leq \frac{C}{(n h)^{2}}\left(\sum_{t=B_{j}+1}^{B_{j}+a} k_{t}(\tau)^{2} u_{t}^{2}\right)^{2}$, and therefore

$$
\begin{aligned}
\sum_{j=1}^{k} \mathbb{E}^{*}\left[\frac{X_{n, j}^{*}(\tau)^{2}}{\omega_{n}^{* 2}} \mathbb{1}\right. & \left.\left(\left|\frac{X_{n, j}^{*}(\tau)^{2}}{\omega_{n}^{* 2}}\right|>\kappa\right)\right] \\
& \leq \frac{C}{(n h)^{2} \omega^{* 4}} \sum_{j=1}^{k}\left(\sum_{t=B_{j}+1}^{B_{j}+a} k_{t}(\tau)^{2} u_{t}^{2},\right)^{2},
\end{aligned}
$$

where $\omega_{n}^{*-4}=O_{p}(1)$. By Minkowski's inequality, stationarity of $u_{t}$, and $\mathbb{E} u_{t}^{4}<\infty$ - let $M \rightarrow \infty$ in $(2.20)$ - we have that

$$
\begin{aligned}
\frac{1}{(n h)^{2}} \sum_{j=1}^{k} \mathbb{E}\left(\sum_{t=B_{j}+1}^{B_{j}+a} k_{t}(\tau)^{2} u_{t}^{2}\right)^{2} & \leq \frac{1}{(n h)^{2}}\left(\mathbb{E} u_{t}^{4}\right) \sum_{i=1}^{k}\left(\sum_{t=B_{j}+1}^{B_{j}+a} k_{t}(\tau)^{2}\right)^{2} \\
& \leq \frac{C_{1} a}{n h}=o(1)
\end{aligned}
$$




\section{A.3 Uniform Results}

Before deriving Theorem 2.3, we propose a few auxiliary lemmas aimed at establishing stochastic equicontinuity, which will be needed to extend the pointwise results to uniformity.

Lemma 2.8. Let $\left\{X_{t}\right\}_{t=1}^{n}$ be a stochastic process with $\limsup _{n \rightarrow \infty} \sum_{i=1}^{n-1} \max _{1 \leq t \leq n-i}\left|\mathbb{E} X_{t} X_{t+i}\right|<\infty$. Then, for any $\tau_{0} \in(0,1)$ and $\tau_{1}, \tau_{2} \in[-1,1]$, there exists an $N>0$ such that for all $n>N$

$$
\left|\frac{1}{\sqrt{n h}} \sum_{t=1}^{n}\left[k_{t}\left(\tau_{0}+\tau_{1} h\right)-k_{t}\left(\tau_{0}+\tau_{2} h\right)\right] X_{t}\right| \leq B_{n}\left|\tau_{1}-\tau_{2}\right|,
$$

where $B_{n}$ is a random variable such that $\sup _{n>N, \tau_{1}, \tau_{2} \in[-1,1]} \mathbb{E} B_{n}^{2}<\infty$.

Proof of Lemma 2.8. See Supplementary Appendix B.

Lemma 2.9. Let $\left\{X_{t}^{*}\right\}_{t=1}^{n}$ be a bootstrap process defined conditionally on a process $\left\{X_{t}\right\}_{t=1}^{n}$ with $\lim \sup _{n \rightarrow \infty} \sum_{i=1}^{n-1} \sup _{1 \leq t \leq n-i}\left|\mathbb{E}^{*} X_{t}^{*} X_{t+i}^{*}\right|<\infty$. Then, for any $\tau_{0} \in(0,1)$ and $\tau_{1}, \tau_{2} \in[-1,1]$, there exists an $N>0$ such that for all $n>N$

$$
\left|\frac{1}{\sqrt{n h}} \sum_{t=1}^{n}\left[k_{t}\left(\tau_{0}+\tau_{1} h\right)-k_{t}\left(\tau_{0}+\tau_{2} h\right)\right] X_{t}^{*}\right| \leq B_{n}^{*}\left|\tau_{1}-\tau_{2}\right|,
$$

where $B_{n}^{*}$ is a random variable such that $\sup _{n>N, \tau_{1}, \tau_{2} \in[-1,1]} \mathbb{E} \mathbb{E}^{*} B_{n}^{* 2}<\infty$.

Proof of Lemma 2.9. See Supplementary Appendix B.

Lemma 2.10. Let $R_{n}(\tau)$ and $R_{n}^{*}(\tau)$ be defined as in Lemmas 2.6 and 2.7 respectively. Then we have, for all $\tau_{0} \in(0,1)$, that $\sup _{\tau \in[-1,1]}\left|R_{n}\left(\tau_{0}+\tau_{1} h\right)\right|=o_{p}(1)$ and $\sup _{\tau \in[-1,1]}\left|R_{n}^{*}\left(\tau_{0}+\tau_{1} h\right)\right|=o_{p}^{*}(1)$.

Proof of Lemma 2.10. See Supplementary Appendix B.

Proof of Theorem 2.3. It follows directly from Lemma 2.10 that

$$
\begin{gathered}
\sup _{\tau \in[-1,1]}\left|Z_{\tau_{0}, n}(\tau)-p\left(\tau_{0}+\tau h\right)^{-1} Z_{n, U}\left(\tau_{0}+\tau h\right)-B_{a s}\left(\tau_{0}\right)\right| \\
\quad \leq \sup _{\tau \in[-1,1]}\left|R_{n}\left(\tau_{0}+\tau h\right)\right|=o_{p}(1),
\end{gathered}
$$


and, equally, that

$$
\begin{gathered}
\sup _{\tau \in[-1,1]}\left|Z_{\tau_{0}, n}^{*}(\tau)-p\left(\tau_{0}+\tau h\right)^{-1} Z_{n, U}^{*}\left(\tau_{0}+\tau h\right)-B_{a s}\left(\tau_{0}\right)\right| \\
\quad \leq \sup _{\tau \in[-1,1]}\left|R_{n}^{*}\left(\tau_{0}+\tau h\right)\right|=o_{p}^{*}(1),
\end{gathered}
$$

such that we only have to consider $p\left(\tau_{0}+\tau h\right)^{-1} Z_{n, U}(\tau)$ and $p\left(\tau_{0}+\tau h\right)^{-1} Z_{n, U}^{*}(\tau)$ in the following.

We next establish the asymptotic covariances. With

$$
\begin{aligned}
\mathbb{E}_{D} Z_{n, U}\left(\tau_{0}+\tau_{1} h\right) Z_{n, U}\left(\tau_{0}+\tau_{2} h\right) \\
\quad=\frac{1}{n h} \sum_{i=-n+1}^{n-1} R_{U}(i) \sum_{t=1}^{n-|i|} k_{t}\left(\tau_{0}+\tau_{1} h\right) k_{t+|i|}\left(\tau_{0}+\tau_{2} h\right) \sigma_{t} \sigma_{t+|i|} D_{t} D_{t+|i|},
\end{aligned}
$$

we apply Lemma 2.4(iii) with $\tilde{g}_{i}\left(\tau_{1}, \tau_{2}\right)=\sigma\left(\tau_{1}\right) \sigma\left(\tau_{2}\right)$ to find that

$$
\begin{aligned}
& \sup _{\tau_{0} \in[\delta, 1-\delta]} \sup _{\tau_{1}, \tau_{2} \in[-1,1]} \| \mathbb{E}_{D} Z_{n, U}\left(\tau_{0}+\tau_{1} h\right) Z_{n, U}\left(\tau_{0}+\tau_{2} h\right) \\
& \quad-p\left(\tau_{0}\right) \sigma^{2}\left(\tau_{0}\right) \kappa\left(\tau_{1}-\tau_{2}\right) \Omega_{U} \| \\
& \leq \sum_{i=-n+1}^{n-1}\left|R_{U}(i)\right|\left[\frac{C_{1}}{\sqrt{n h}}+\frac{C_{2}}{\sqrt{n h}} \max \left\{h, \frac{1}{n h^{2}}\right\}+S_{n}(i)\right] \\
& \quad+\sum_{i=-\infty}^{\infty} \mathbb{1}(|i| \geq n)\left|R_{U}(i)\right|, \\
& \leq \frac{C_{3}}{\sqrt{n h}}+\frac{C_{3}}{\sqrt{n h}} \max \left\{h, \frac{1}{n h^{2}}\right\}+\phi_{n},
\end{aligned}
$$

from which it follows by the law of iterated expectations that

$$
\begin{aligned}
& \sup _{\tau \in[\delta, 1-\delta]} \sup _{\tau_{1}, \tau_{2} \in[-1,1]}\left|\mathbb{E} Z_{n, U}\left(\tau_{0}+\tau_{1} h\right) Z_{n, U}\left(\tau_{0}+\tau_{2} h\right)-p\left(\tau_{0}\right) \sigma_{W, \tau_{0}}\left(\tau_{1}, \tau_{2}\right)\right| \\
& \quad=o(1) .
\end{aligned}
$$

It then follows that

$$
\begin{aligned}
& \sup _{\tau \in[\delta, 1-\delta]} \sup _{\tau_{1}, \tau_{2} \in[-1,1]}\left|\mathbb{E} Z_{\tau_{0}, n}\left(\tau_{1}\right) Z_{\tau_{0}, n}\left(\tau_{2}\right)-\sigma_{W, \tau_{0}}\left(\tau_{1}, \tau_{2}\right)\right| \\
& \leq \sup _{\tau \in[\delta, 1-\delta]} \sup _{\tau_{1}, \tau_{2} \in[-1,1]} \mid \mathbb{E} Z_{\tau_{0}, n}\left(\tau_{1}\right) Z_{\tau_{0}, n}\left(\tau_{2}\right)-p\left(\tau_{0}+\tau h\right)^{-1} p\left(\tau_{0}+\tau_{2} h\right)^{-1}
\end{aligned}
$$




$$
\begin{aligned}
& \times p\left(\tau_{0}\right)^{2} \sigma_{W, \tau_{0}}\left(\tau_{1}, \tau_{2}\right)\left|+\sup _{\tau \in[\delta, 1-\delta]} \sup _{\tau_{1}, \tau_{2} \in[-1,1]}\right| p\left(\tau_{0}+\tau_{1} h\right)^{-1} p\left(\tau_{0}+\tau_{2} h\right)^{-1} \\
& \times p\left(\tau_{0}\right)^{2}-1 \mid \sigma_{W, \tau_{0}}\left(\tau_{1}, \tau_{2}\right) \\
\leq & o(1)+o(h)=o(1) .
\end{aligned}
$$

We follow the same steps as in the proof of the asymptotic bootstrap variance in Lemma 2.7 for the bootstrap covariances. Note that

$$
\begin{aligned}
\mathbb{E}^{*} Z_{n, U}^{*}\left(\tau_{0}+\tau_{1} h\right) Z_{n, U}^{*}\left(\tau_{0}+\tau_{2} h\right) \\
\quad=\frac{1}{n h} \sum_{i=1-n}^{n-1} \sum_{t=1}^{n-|i|} k_{t}\left(\tau_{0}+\tau_{1} h\right) k_{t+i}\left(\tau_{0}+\tau_{2} h\right) D_{t} D_{t+i} z_{t} z_{t+i} \gamma^{i},
\end{aligned}
$$

where it follows from a straightforward adaptation of Lemma 2.5 allowing for different $\tau$ 's that

$$
\begin{aligned}
& \mathbb{E}\left|\mathbb{E}^{*} Z_{n, U}^{*}\left(\tau_{0}+\tau_{1} h\right) Z_{n, U}^{*}\left(\tau_{0}+\tau_{2} h\right)-\mathbb{E}_{D} \mathbb{E}^{*} Z_{n, U}^{*}\left(\tau_{0}+\tau_{1} h\right) Z_{n, U}^{*}\left(\tau_{0}+\tau_{2} h\right)\right| \\
& \quad=o(1) .
\end{aligned}
$$

To conclude this part, we can show as in Lemma 2.7 that

$$
\begin{aligned}
& \sup _{\tau_{0} \in\left[\delta^{*}, 1-\delta^{*}\right]} \sup _{\tau_{1}, \tau_{2} \in[-1,1]} \mid \mathbb{E}_{D} \mathbb{E}^{*} Z_{n, U}^{*}\left(\tau_{0}+\tau_{1} h\right) Z_{n, U}^{*}\left(\tau_{0}+\tau_{2} h\right) \\
&-\mathbb{E}_{D} Z_{n, U}\left(\tau_{0}+\tau_{1} h\right) Z_{n, U}\left(\tau_{0}+\tau_{2} h\right) \mid=o_{p}(1) .
\end{aligned}
$$

Finite-dimensional convergence of the vectors $\left(Z_{\tau_{0}, n}\left(\tau_{1}\right), \ldots, Z_{\tau_{0}, n}\left(\tau_{m}\right)\right)^{\prime}$ and $\left(Z_{\tau_{0}, n}^{*}\left(\tau_{1}\right), \ldots, Z_{\tau_{0}, n}^{*}\left(\tau_{m}\right)\right)^{\prime}$ for $\left(\tau_{1}, \ldots, \tau_{m}\right)^{\prime} \in[-1,1]^{m}$ follows from Theorems 2.1 and 2.2 and the Cramér-Wold device; it remains to show tightness. By applying Lemma 2.8 with $X_{t}=D_{t} z_{t}$ and Lemma 2.9 with $X_{t}^{*}=D_{t} z_{t} \xi_{t}^{*}$, it follows directly that

$$
\begin{aligned}
& \mathbb{E}\left(Z_{\tau_{0}, n}\left(\tau_{1}\right)-Z_{\tau_{0}, n}\left(\tau_{2}\right)\right)^{2} \leq \mathbb{E} B_{n}^{2}\left|\tau_{1}-\tau_{2}\right|^{2}, \\
& \mathbb{E} \mathbb{E}^{*}\left(Z_{\tau_{0}, n}\left(\tau_{1}\right)-Z_{\tau_{0}, n}\left(\tau_{2}\right)\right)^{2} \leq \mathbb{E} \mathbb{E}^{*} B_{n}^{* 2}\left(\tau_{1}-\tau_{2}\right)^{2},
\end{aligned}
$$

where $\mathbb{E} B_{n}^{2} \leq C$ and $\mathbb{E} \mathbb{E}^{*} B_{n}^{* 2} \leq C$ and tightness follows by Theorem 12.3 of Billingsley (1968). 


\section{B Additional Proofs}

In this appendix we prove the auxiliary lemmas not proven in the main part.

Proof of Lemma 2.1. For $(i)$, note that, by the Cauchy-Schwarz inequality and Assumption 2.4, we have that

$$
\begin{aligned}
\sum_{i=0}^{n-1} i \sup _{t}\left|\operatorname{Cov}\left(D_{t}, D_{t+i}\right)\right| & =\sum_{i=0}^{n-1} i \sup _{t}\left|\mathbb{E}\left\{\left[D_{t}-\mathbb{E} D_{t}\right] \mathbb{E}\left[D_{t+i}-\mathbb{E} D_{t+i} \mid \mathcal{F}_{t}\right]\right\}\right| \\
& \leq \sum_{i=0}^{n-1} i \sup _{t}\left\|D_{t}-\mathbb{E} D_{t}\right\|\left\|\mathbb{E}\left[D_{t+i}-\mathbb{E} D_{t+i} \mid \mathcal{F}_{t}\right]\right\| \\
& \leq \sum_{i=0}^{n-1} i C \zeta_{i} \leq C \sum_{i=0}^{\infty} i \zeta_{i}<\infty
\end{aligned}
$$

For $($ ii $)$, it follows from Assumption 2.3 that

$$
\begin{aligned}
\sum_{i=1}^{n-1} i \sup _{t}\left|\mathbb{C o v}\left(u_{t}, u_{t+i}\right)\right| & =\sum_{i=1}^{n-1} i\left|R_{U}(i)\right|=\sigma_{\varepsilon}^{2} \sum_{i=1}^{n-1} i\left|\sum_{j=0}^{\infty} \psi_{j} \psi_{j+|k|}\right| \\
& \leq \sum_{i=0}^{\infty} \sum_{j=0}^{\infty} i\left|\psi_{i}\right|\left|\psi_{j}\right|=\left(\sum_{i=0}^{\infty} i\left|\psi_{i}\right|\right)\left(\sum_{j=0}^{\infty}\left|\psi_{j}\right|\right)<\infty
\end{aligned}
$$

Finally for $(i i i)$, as $0 \leq D_{t} \leq 1$ for all $t$, it follows that $\left|\mathbb{E} D_{t} D_{t+i} u_{t} u_{t+i}\right| \leq$ $\left|\mathbb{E} u_{t} u_{t+i}\right|$, such that the result directly follows from $(i i)$.

Proof of Lemma 2.2. By the compact support of $K(\cdot), k_{t}(\tau)=0$ if $|t / n-\tau|>$ $C_{K} h$ for some $C_{K}>0$. Therefore

$$
\begin{aligned}
& \sup _{\tau \in[\delta, 1-\delta]} \max _{0 \leq i \leq n} \frac{1}{n h} \sum_{t=1}^{n} k_{t}(\tau) k_{t+i}(\tau) \leq \frac{1}{n h} \sup _{\tau \in[\delta, 1-\delta]} \sup _{\omega} K(\omega) \sum_{t=n\left(\tau-C_{K} h\right)}^{n\left(\tau+C_{K} h\right)} k_{t}(\tau) \\
& \leq \frac{1}{n h} \sup _{\omega} K(\omega)^{2} 2 C_{K} n h \leq C .
\end{aligned}
$$


Proof of Lemma 2.3. For part $(i)$, note that

$$
\begin{aligned}
& \frac{1}{n h} \sum_{t=1}^{n} f\left(\frac{t}{n}\right) k_{t}(\tau)-f(\tau) \kappa_{1} \\
& =\left[\frac{1}{n h} \sum_{t=1}^{n} f\left(\frac{t}{n}\right) k_{t}(\tau)-h^{-1} \int_{0}^{1} f(x) K\left(\frac{x-\tau}{h}\right) \mathrm{d} x\right] \\
& \quad+\left[h^{-1} \int_{0}^{1} f(x) K\left(\frac{x-\tau}{h}\right) \mathrm{d} x-f(\tau) \kappa_{1}\right] \\
& =I_{1, n}(\tau)+I_{2, n}(\tau) .
\end{aligned}
$$

We first consider $I_{1, n}(\tau)$. For a continuous and Riemann-integrable function $g(\cdot)$, we have the integral approximation bound (cf. Bühlmann, 1998, p. 79, (6.5))

$$
\left|\frac{1}{n} \sum_{t=1}^{n} g\left(\frac{t}{n}\right)-\int_{0}^{1} g(z) d z\right| \leq \sup _{|x-y| \leq n^{-1}}|g(x)-g(y)| .
$$

Take $g(x)=\frac{1}{h} f(x) K\left(\frac{x-\tau}{h}\right)$, then the bound yields

$$
\begin{aligned}
\left|I_{1, n}(\tau)\right| & \leq \sup _{|x-y| \leq n^{-1}} h^{-1}\left|K\left(\frac{x-\tau}{h}\right) f(x)-K\left(\frac{y-\tau}{h}\right) f(y)\right| \\
& \leq \sup _{|x-y| \leq n^{-1}}\left[K\left(\frac{x-\tau}{h}\right) \frac{|x-y|}{h}+f(y) \frac{|x-y|}{h^{2}}\right] \leq \frac{C_{1}}{n h}+\frac{C_{2}}{n h^{2}} .
\end{aligned}
$$

For $I_{2, n}(\tau)$ perform a change of variables with $u=(x-\tau) / h$ such that $\int_{0}^{1} K\left(\frac{x-\tau}{h}\right) f(x) d x=\int_{-\tau / h}^{(1-\tau) / h} K(u) f(\tau+u h) d u$. Take an $N$ such that for all $n>N, \min \{\tau / h,(1-\tau) / h\}$ will be larger than the bounds of the compact support of $K(\cdot)$; it then follows that $\int_{-\tau / h}^{(1-\tau) / h} K(u) f(u h+\tau) d u=\int_{\mathbb{R}} K(u) f(\tau+u h) d u$ for all $n>N$. Then a Taylor expansion of $f(\tau+u h)$ around $f(\tau)$ yields

$$
f(\tau+u h)=f(\tau)+f^{(1)}(\tau) u h+\frac{1}{2} f^{(2)}\left(\tau^{*}\right)(u h)^{2},
$$

where $\left|\tau^{*}-\tau\right| \leq u h$. As $\mu_{1}=\int_{\mathbb{R}} K(u) u \mathrm{~d} u=0$, it follows that

$$
\begin{aligned}
\int_{\mathbb{R}} K(u) f(\tau+u h) \mathrm{d} u= & f(\tau) \int_{\mathbb{R}} K(u) \mathrm{d} u+f^{(1)}(\tau) h \int_{\mathbb{R}} K(u) u \mathrm{~d} u \\
& +\frac{1}{2} h^{2} \int_{\mathbb{R}} f^{(2)}\left(\tau^{*}\right) K(u) u^{2} \mathrm{~d} u \\
= & f(\tau) \kappa_{1}+\frac{1}{2} h^{2} \int_{\mathbb{R}} f^{(2)}\left(\tau^{*}\right) K(u) u^{2} \mathrm{~d} u .
\end{aligned}
$$


It then follows from the Lipschitz continuity of $f^{(2)}(\cdot)$ that $\left|f^{(2)}\left(\tau^{*}\right)-f^{(2)}(\tau)\right| \leq$ Cuh and consequently

$$
\begin{aligned}
\left|I_{2, n}(\tau)\right| \leq & \left|\int_{\mathbb{R}} K(u) f(u h+\tau) d u-f(\tau) \kappa_{1}-\frac{1}{2} h^{2} \int_{\mathbb{R}} f^{(2)}\left(\tau^{*}\right) K(u) u^{2} \mathrm{~d} u\right| \\
& +\frac{1}{2} h^{2}\left|\int_{\mathbb{R}}\left[f^{(2)}\left(\tau^{*}\right)-f^{(2)}(\tau)\right] K(u) u^{2} \mathrm{~d} u\right| \\
\leq & 0+\frac{C}{2} h^{3} \int_{\mathbb{R}} K(u)|u|^{3} \mathrm{~d} u \leq C_{1} h^{3},
\end{aligned}
$$

as $\int_{\mathbb{R}} K(u)|u|^{3} \mathrm{~d} u<\infty$ by the compact support of $K(\cdot)$. As none of the bounds depend on $\tau$, and the Taylor expansion is appropriate for any $\tau \in(0,1)$, the result follows.

For part $(i i)$, we can write

$$
\begin{aligned}
& \frac{1}{n h} \sum_{t=1}^{n-i} g\left(\frac{t}{n}, \frac{t+i}{n}\right) k_{t}\left(\tau_{0}+\tau_{1} h\right) k_{t+i}\left(\tau_{0}+\tau_{2} h\right)-g\left(\tau_{0}, \tau_{0}\right) \kappa\left(\tau_{1}-\tau_{2}\right) \\
& =\frac{1}{n h} \sum_{t=1}^{n-i} k_{t}\left(\tau_{0}+\tau_{1} h\right)\left[g\left(\frac{t}{n}, \frac{t+i}{n}\right) k_{t+i}\left(\tau_{0}+\tau_{2} h\right)-g\left(\frac{t}{n}, \frac{t}{n}\right) k_{t}\left(\tau_{0}+\tau_{2} h\right)\right] \\
& \quad+\frac{1}{n h} \sum_{t=1}^{n-i} g\left(\frac{t}{n}, \frac{t}{n}\right) k_{t}\left(\tau_{0}+\tau_{1} h\right) k_{t}\left(\tau_{0}+\tau_{2} h\right)-g\left(\tau_{0}, \tau_{0}\right) \kappa\left(\tau_{1}-\tau_{2}\right) \\
& =I I_{1, n}(\tau)+I I_{2, n}(\tau),
\end{aligned}
$$

where $\tau=\left(\tau_{0}, \tau_{1}, \tau_{2}\right)^{\prime}$. Then

$$
\begin{aligned}
\left|I I_{1, n}(\tau)\right| \leq & \frac{1}{n h} \sum_{t=1}^{n-i} k_{t}\left(\tau_{0}+\tau_{1} h\right)\left\{\left|g\left(\frac{t}{n}, \frac{t+i}{n}\right)\right|\left|k_{t+i}\left(\tau_{0}+\tau_{2} h\right)-k_{t}\left(\tau_{0}+\tau_{2} h\right)\right|\right. \\
& \left.+k_{t}\left(\tau_{0}+\tau_{2} h\right)\left|g\left(\frac{t}{n}, \frac{t}{n}\right)-g\left(\frac{t}{n}, \frac{t+i}{n}\right)\right|\right\} \\
\leq & \frac{C_{g}}{n h} \sum_{t=1}^{n} k_{t}\left(\tau_{0}+\tau_{1} h\right)\left(\frac{i}{n h}+\frac{i}{n}\right) \leq C_{g} C_{1} \frac{i}{n h}
\end{aligned}
$$

and

$$
\left|I I_{2, n}(\tau)\right|=\frac{1}{n h} \sum_{t=1}^{n-i} k_{t}\left(\tau_{0}+\tau_{1} h\right) k_{t}\left(\tau_{0}+\tau_{2} h\right)\left[g\left(\frac{t}{n}, \frac{t}{n}\right)-g\left(\tau_{0}, \tau_{0}\right)\right]
$$




$$
\begin{aligned}
& +\frac{1}{h} g\left(\tau_{0}, \tau_{0}\right)\left[\frac{1}{n} \sum_{t=1}^{n-i} k_{t}\left(\tau_{0}+\tau_{1} h\right) k_{t}\left(\tau_{0}+\tau_{2} h\right)\right. \\
& \left.\quad-\int_{0}^{1} K\left(\frac{x-\tau_{0}-\tau_{1} h}{h}\right) K\left(\frac{x-\tau_{0}-\tau_{2} h}{h}\right) \mathrm{d} x\right] \\
& +g\left(\tau_{0}, \tau_{0}\right)\left[h^{-1} \int_{0}^{1} K\left(\frac{x-\tau_{0}-\tau_{1} h}{h}\right) K\left(\frac{x-\tau_{0}-\tau_{2} h}{h}\right) \mathrm{d} x\right. \\
& \left.\quad-\kappa\left(\tau_{1}-\tau_{2}\right)\right] \\
& =I I_{21, n}(\tau)+I I_{22, n}(\tau)+I I_{23, n}(\tau) .
\end{aligned}
$$

By the compact support of $K(\cdot), k_{t}\left(\tau_{0}+\tau_{1} h\right)>0$ only if $\left|t / n-\tau_{0}-\tau_{1} h\right| \leq C_{K} h$ for some $C_{K}>0$, which implies that $\left|t / n-\tau_{0}\right| \leq C h$. Therefore,

$$
\begin{aligned}
\left|I I_{21, n}(\tau)\right| & \leq \frac{1}{n h} \sum_{t=1}^{n} k_{t}\left(\tau_{0}+\tau_{1} h\right) k_{t}\left(\tau_{0}+\tau_{2} h\right)\left|g\left(\frac{t}{n}, \frac{t}{n}\right)-g\left(\tau_{0}, \tau_{0}\right)\right| \\
& \leq \frac{C_{g}}{n h} \sum_{t=1}^{n} k_{t}\left(\tau_{0}+\tau_{1} h\right) k_{t}\left(\tau_{0}+\tau_{2} h\right)\left|t / n-\tau_{0}\right| \leq C_{g} C_{2} h .
\end{aligned}
$$

For $I I_{22, n}(\tau)$, we again apply the integral approximation bound (2.25) to find

$$
\begin{aligned}
& \left|I I_{22, n}(\tau)\right| \leq \frac{1}{h} g(\tau, \tau) \mid \frac{1}{n} \sum_{t=1}^{n} k_{t}\left(\tau_{0}+\tau_{1} h\right) k_{t}\left(\tau_{0}+\tau_{2} h\right) \\
& -\int_{0}^{1} K\left(\frac{x-\tau_{0}-\tau_{1} h}{h}\right) K\left(\frac{x-\tau_{0}-\tau_{2} h}{h}\right) \mathrm{d} x \mid \\
& +\frac{1}{n h} g(\tau, \tau) \sum_{t=n-i}^{n} k_{t}\left(\tau_{0}+\tau_{1} h\right) k_{t}\left(\tau_{0}+\tau_{2} h\right) \\
& \leq \frac{C_{g}}{h} \sup _{|x-y| \leq \frac{1}{n}} \mid K\left(\frac{x-\tau_{0}-\tau_{1} h}{h}\right) K\left(\frac{x-\tau_{0}-\tau_{2} h}{h}\right) \\
& -K\left(\frac{y-\tau_{0}-\tau_{1} h}{h}\right) K\left(\frac{y-\tau_{0}-\tau_{2} h}{h}\right) \mid \\
& +\frac{C_{g}}{n h} \sum_{t=n-i}^{n} k_{t}\left(\tau_{0}+\tau_{1} h\right) k_{t}\left(\tau_{0}+\tau_{2} h\right) \leq \frac{C_{g} C_{3}}{n h^{2}}+C_{g} S_{n}(i),
\end{aligned}
$$

where $S_{n}(i)=\frac{1}{n h} \sup _{\tau \in[\delta, 1-\delta]} \sum_{t=n-i}^{n} k_{t}(\tau)^{2}$. Now take a sequence $M=M(n)$ such that $M \rightarrow \infty$ as $n \rightarrow \infty$ and $M / n \rightarrow 0$. Then take an $N_{1}$ such that for all 
$n>N_{1}, M / n \leq \delta-\epsilon$ for some $\epsilon>0$, such that $k_{t}(\tau)=0$ for all $t \leq M$ and $\tau \in[\delta, 1-\delta]$. Then for all $n>N_{1}$,

$$
\sum_{i=1}^{n-1} \beta_{i} S_{n}(i) \leq \frac{1}{n h} \sum_{i=M+1}^{\infty} \beta_{i} \sup _{\tau \in[\delta, 1-\delta]} \sum_{t=1}^{n} k_{t}(\tau)^{2} \leq C \sum_{i=M+1}^{\infty} \beta_{i} .
$$

As $M \rightarrow \infty$ and $\sum_{i=1}^{\infty} \beta_{i}<\infty, \phi_{n}=C \sum_{i=M+1}^{\infty} \beta_{i}=o(1)$.

Finally, for $I I_{23, n}(\tau)$ perform a change of variables with $u=\left(x-\tau_{0}\right) / h$ such that

$$
\begin{gathered}
\frac{1}{h} \int_{0}^{1} K\left(\frac{x-\tau_{0}-\tau_{1} h}{h}\right) K\left(\frac{x-\tau_{0}-\tau_{2} h}{h}\right) \mathrm{d} x \\
\quad=\int_{-\tau_{0} / h}^{\left(1-\tau_{0}\right) / h} K\left(u-\tau_{1}\right) K\left(u-\tau_{2}\right) \mathrm{d} u
\end{gathered}
$$

Take an $N_{2}$ such that for all $n>N_{2}, \min \left\{\tau_{0} / h,\left(1-\tau_{0}\right) / h\right\}$ will be larger than the bounds of the compact support of $K(\cdot)$ such that

$$
\int_{-\tau_{0} / h}^{\left(1-\tau_{0}\right) / h} K\left(u-\tau_{1}\right) K\left(u-\tau_{2}\right) \mathrm{d} u=\int_{-\infty}^{\infty} K\left(u-\tau_{1}\right) K\left(u-\tau_{2}\right) \mathrm{d} u
$$

for all $n>N_{2}$. Finally, a second change of variables with $\omega=u-\tau_{1}$ shows that

$$
\int_{-\infty}^{\infty} K\left(u-\tau_{1}\right) K\left(u-\tau_{2}\right) \mathrm{d} u=\kappa_{2}\left(\tau_{1}-\tau_{2}\right)
$$

and thus $I I_{23, n}(\tau)=0$ for $n>N_{2}$. As no bounds depend on $\tau_{0}, \tau_{1}$ or $\tau_{2}$, all results hold uniformly for $n>N=\max \left\{N_{1}, N_{2}\right\}$.

Proof of Lemma 2.4. For part $(i)$, write $\bar{f}_{n}\left(\tau_{1}, \tau_{2}\right)=f\left(\tau_{1}\right)-l_{n}\left(\tau_{2}\right)$.

$$
\begin{aligned}
& \mathbb{E} Z_{n, D, f}(\tau)^{2}=\frac{1}{n h} \sum_{s=1}^{n} \sum_{t=1}^{n} \bar{f}_{n}\left(\frac{s}{n}, \tau\right) \bar{f}_{n}\left(\frac{t}{n}, \tau\right) k_{s}(\tau) k_{t}(\tau) \operatorname{Cov}\left(D_{s}, D_{t}\right) \\
& =\frac{1}{n h} \sum_{i=-n+1}^{n-1} \sum_{t=1}^{n-|i|} k_{t}(\tau) k_{t+|i|}(\tau) \bar{f}\left(\frac{t}{n}, \tau\right) \bar{f}\left(\frac{t+i}{n}, \tau\right) R_{D,|i|}\left(\frac{t}{n}, \frac{t+i}{n}\right) \\
& =\bar{f}_{n}(\tau, \tau) \Omega_{D}(\tau) \kappa_{2}+A_{n}(\tau),
\end{aligned}
$$


where

$$
\begin{aligned}
A_{n}(\tau)= & 2 \kappa_{2} \bar{f}_{n}(\tau, \tau) \sum_{i=n}^{\infty} R_{D, i}(\tau, \tau)+\sum_{i=-n+1}^{n-1}\left[\frac{1}{n h} \sum_{t=1}^{n-|i|} k_{t}(\tau) k_{t+|i|}(\tau) \bar{f}_{n}\left(\frac{t}{n}, \tau\right)\right. \\
& \left.\times \bar{f}_{n}\left(\frac{t+|i|}{n}, \tau\right) R_{D,|i|}\left(\frac{t}{n}, \frac{t+|i|}{n}\right)-\bar{f}_{n}(\tau, \tau)^{2} R_{D,|i|}(\tau, \tau) \kappa_{2}\right] .
\end{aligned}
$$

Letting $A_{n}(t, i, \tau)=k_{t}(\tau) k_{t+i}(\tau) R_{D, i}\left(\frac{t}{n}, \frac{t+i}{n}\right)$ and $A(i, \tau)=2 \kappa_{2} R_{D, i}(\tau, \tau)$, we can decompose the inner sum as

$$
\begin{aligned}
& \left|\frac{1}{n h} \sum_{t=1}^{n-i} \bar{f}_{n}\left(\frac{t}{n}, \tau\right) \bar{f}_{n}\left(\frac{t+i}{n}, \tau\right) A_{n}(t, i, \tau)-\bar{f}_{n}(\tau, \tau)^{2} A(i, \tau)\right| \\
& \leq\left|\frac{1}{n h} \sum_{t=1}^{n-i} f\left(\frac{t}{n}\right) f\left(\frac{t+i}{n}\right) A_{n}(t, i, \tau)-f(\tau)^{2} A(i, \tau)\right| \\
& +\left|l_{n}(\tau)\right|\left|\frac{1}{n h} \sum_{t=1}^{n-i} f\left(\frac{t}{n}\right) A_{n}(t, i, \tau)-f(\tau) A(i, \tau)\right| \\
& +\left|l_{n}(\tau)\right| \frac{1}{n h} \sum_{t=1}^{n-i} f\left(\frac{t+i}{n}\right) A_{n}(t, i, \tau)-f(\tau) A(i, \tau) \mid \\
& +l_{n}(\tau)^{2}\left|\frac{1}{n h} \sum_{t=1}^{n-i} A_{n}(t, i, \tau)-A(i, \tau)\right|
\end{aligned}
$$

By applying Lemma 2.3(ii) to each of these terms, with $g_{i}\left(\tau_{1}, \tau_{2}\right)=f\left(\tau_{1}\right) f\left(\tau_{2}\right) R_{D, i}\left(\tau_{1}, \tau_{2}\right)$ for the first term and defined analogously for the rest, we find that

$$
\begin{aligned}
\sup _{\tau \in[\delta, 1-\delta]}\left|A_{n}(\tau)\right| \leq & C_{1} \sum_{i=1}^{\infty} \sup _{\tau \in[0,1]}\left|R_{D, i}(\tau, \tau)\right|+\frac{2}{n h} C_{1} \sum_{i=1}^{n-1} i C_{g}(i) \\
& +C_{2}\left\{h, \frac{1}{n h^{2}}\right\} \sum_{i=1}^{n-1} C_{g}(i)+\sum_{i=1}^{n-1} C_{g}(i) S_{n}(i) \\
\leq & C \max \left\{h, \frac{1}{n h^{2}}\right\}+\phi_{n},
\end{aligned}
$$

with $C_{g}(i)=\sup _{\left(\tau_{1}, \tau_{2}\right) \in[0,1]^{2}}\left|R_{D, i}\left(\tau_{1}, \tau_{2}\right)\right| \max \left\{\sup _{\tau \in[0,1]} f(\tau)^{2}, 1\right\}$, where

$$
\sum_{i=0}^{\infty} i C_{g}(i) \leq C \sum_{i=0}^{\infty} i \sup _{\left(\tau_{1}, \tau_{2}\right)}\left|R_{D, i}\left(\tau_{1}, \tau_{2}\right)\right|<\infty
$$




$$
\lim _{n \rightarrow \infty} \phi_{n}=C_{1} \lim _{n \rightarrow \infty} \sum_{i=n}^{\infty} \sup _{\tau \in[0,1]}\left|R_{D, i}(\tau, \tau)\right|+\lim _{n \rightarrow \infty} \sum_{i=1}^{n-1} C_{g}(i) S_{n}(i)=0 .
$$

Part (ii) follows directly by noting that

$$
\begin{aligned}
\frac{1}{n h} \sum_{t=1}^{n} f\left(\frac{t}{n}\right) k_{t}(\tau) D_{t}-p(\tau) f(\tau)-h^{2} p^{(2)}(\tau) \mu_{2} \\
=\left[\frac{1}{n h} \sum_{t=1}^{n} f\left(\frac{t}{n}\right) k_{t}(\tau)\left[D_{t}-p\left(\frac{t}{n}\right)\right]\right] \\
\quad+\left[\frac{1}{n h} \sum_{t=1}^{n} f\left(\frac{t}{n}\right) p\left(\frac{t}{n}\right) k_{t}(\tau)-p(\tau) f(\tau)-h^{2}[f p]^{(2)}(\tau) \mu_{2}\right] \\
=Z_{n, D, f, 0}(\tau)+R_{n, D}(\tau),
\end{aligned}
$$

as it follows directly from Lemma $2.3(i)$ that

$$
\sup _{\tau \in[\delta, 1-\delta]}\left|R_{n, D}(\tau)\right|<C \max \left\{h^{3}, \frac{1}{n h}\right\} \text {. }
$$

For part $(i i i)$, we can write

$$
\begin{aligned}
& \frac{1}{n h} \sum_{t=1}^{n-i} \tilde{g}_{i}\left(\frac{t}{n}, \frac{t+i}{n}\right) k_{t}\left(\tau_{0}+\tau_{1} h\right) k_{t+i}\left(\tau_{0}+\tau_{2} h\right) D_{t} D_{t+i}-p\left(\tau_{0}\right) \tilde{g}_{i}\left(\tau_{0}, \tau_{0}\right) \kappa\left(\tau_{1}-\tau_{2}\right) \\
& =\left[\frac{1}{n h} \sum_{t=1}^{n-i} \tilde{g}_{i}\left(\frac{t}{n}, \frac{t+i}{n}\right) k_{t}\left(\tau_{0}+\tau_{1} h\right) k_{t+i}\left(\tau_{0}+\tau_{2} h\right)\left[D_{t} D_{t+i}-\mathbb{E} D_{t} D_{t+i}\right]\right] \\
& +\left[\frac{1}{n h} \sum_{t=1}^{n-i} \tilde{g}_{i}\left(\frac{t}{n}, \frac{t+i}{n}\right) k_{t}\left(\tau_{0}+\tau_{1} h\right) k_{t+i}\left(\tau_{0}+\tau_{2} h\right) \mathbb{E} D_{t} D_{t+i}\right. \\
& \left.\quad-p\left(\tau_{0}\right) \tilde{g}_{i}\left(\tau_{0}, \tau_{0}\right) \kappa\left(\tau_{1}-\tau_{2}\right)\right] \\
& =I I I_{n, 1}(\tau)+I I I_{n, 2}(\tau) .
\end{aligned}
$$

Define $X_{t}(i)=k_{t-i}\left(\tau_{0}+\tau_{1} h\right) k_{t}\left(\tau_{0}+\tau_{2} h\right) \tilde{g}_{i}\left(\frac{t-i}{n}, \frac{t}{n}\right)\left[D_{t-i} D_{t}-\mathbb{E}\left(D_{t-i} D_{t}\right)\right]$; it then follows from Assumption 2.4 that $X_{t}(i)$ is an $L_{2^{-}}$mixingale with $\left\|\mathbb{E}_{t-j} X_{t}(i)\right\| \leq$ $k_{t-i}\left(\tau_{0}+\tau_{1} h\right) k_{t}\left(\tau_{0}+\tau_{2} h\right) C_{g}(i) \zeta_{j}$. Then, by Theorem 1.6 of McLeish (1975) and 
Lemma 2.3,

$$
\begin{aligned}
\mathbb{E}\left|I I I_{n, 1}(\tau)\right| & \leq \frac{1}{n h} \mathbb{E}\left|\sum_{t=i+1}^{n} X_{t}(i)\right| \\
& \leq \frac{C}{n h}\left(\sum_{t=1}^{n-i} k_{t-i}\left(\tau_{0}+\tau_{1} h\right)^{2} k_{t}\left(\tau_{0}+\tau_{2} h\right)^{2} C_{g}(i)^{2}\right)^{1 / 2} \\
& \leq \frac{C}{n h}\left(C_{g}(i)^{2} \sum_{t=i+1}^{n} \sup _{\tau \in[\delta, 1-\delta]} k_{t-i}(\tau)^{2} k_{t}(\tau)^{2}\right)^{1 / 2} \leq \frac{C_{1}}{\sqrt{n h}} C_{g}(i) .
\end{aligned}
$$

Finally, as $\mathbb{E} D_{t} D_{t+i}=p\left(\frac{t}{n}\right) p\left(\frac{t+i}{n}\right)+R_{D, i}\left(\frac{t}{n}, \frac{t+i}{n}\right)$, it follows directly from Lemma $2.3(i i)$ that

$$
\sup _{\tau \in[\delta, 1-\delta]}\left|I I I_{n, 2}(\tau)\right| \leq C_{g}(i)\left[\frac{C_{1}}{n h}+C_{2} \max \left\{h, \frac{1}{n h^{2}}\right\}+S_{n}(i)\right]
$$

by taking $g_{i}\left(\tau_{1}, \tau_{2}\right)=\tilde{g}_{i}\left(\tau_{1}, \tau_{2}\right)\left[p\left(\frac{t}{n}\right) p\left(\frac{t+i}{n}\right)+R_{D, i}\left(\frac{t}{n}, \frac{t+i}{n}\right)\right]$.

Proof of Lemma 2.5. The proof is an extension of the proof of Lemma 5 in Jansson (2002). As $\mathbb{E} u_{t} u_{t+i}=\sigma_{\varepsilon}^{2} \sum_{j} \psi_{j} \psi_{j+i}$, we can write

$$
\begin{aligned}
A_{n}(\tau)= & \frac{1}{n h} \sum_{t=1}^{n-i} k_{t}(\tau) k_{t+i}(\tau) \sigma_{t} \sigma_{t+i} D_{t} D_{t+i}\left[u_{t} u_{t+i}-\mathbb{E} u_{t} u_{t+i}\right] \\
= & \sigma_{\varepsilon}^{2} \sum_{j=0}^{\infty} \psi_{j} \psi_{j+i}\left[\frac{1}{n h} \sum_{t=1}^{n-i} k_{t}(\tau) k_{t+i} D_{t} D_{t+i}(\tau) \sigma_{t} \sigma_{t+i}\left(\varepsilon_{t-j}^{2}-1\right)\right] \\
& +\sigma_{\varepsilon}^{2} \sum_{j=0}^{\infty} \sum_{m=0}^{\infty} \mathbb{1}(m \neq j+i) \psi_{j} \psi_{m} \frac{1}{n h} \sum_{t=1}^{n-i} k_{t}(\tau) k_{t+i}(\tau) \\
& \times D_{t} D_{t+i} \sigma_{t} \sigma_{t+i} \varepsilon_{t-j} \varepsilon_{t+i-m} .
\end{aligned}
$$


Then

$$
\begin{aligned}
& \mathbb{E}\left|A_{n}(\tau)\right| \\
& \leq \sigma_{\varepsilon}^{2} \sup _{j \geq 0} \max _{0 \leq i \leq n-1} \mathbb{E}\left|\frac{1}{n h} \sum_{t=1}^{n-i} D_{t} D_{t+i} k_{t}(\tau) k_{t+i}(\tau) \sigma_{t} \sigma_{t+i}\left(\varepsilon_{t-j}^{2}-1\right)\right|\left(\sum_{j=0}^{\infty}\left|\psi_{j}\right|\left|\psi_{j+i}\right|\right) \\
& \quad+\sigma_{\varepsilon}^{2} \frac{1}{\sqrt{n h}} \sup _{j, m \geq 0} \max _{0 \leq i \leq n-1} \mathbb{E} \mid \frac{1}{\sqrt{n h}} \mathbb{1}(m \neq j+i) \sum_{t=1}^{n-i} D_{t} D_{t+i} k_{t}(\tau) k_{t+i}(\tau) \sigma_{t} \sigma_{t+i} \\
& \quad \times \varepsilon_{t-j} \varepsilon_{t+i-m}\left|\sum_{j=0}^{\infty} \sum_{m=0}^{\infty}\right| \psi_{j}|| \psi_{m} \mid \\
& =\phi_{n}(\tau) \beta_{i}+\frac{1}{\sqrt{n h}} \eta_{n}(\tau),
\end{aligned}
$$

where

$$
\begin{aligned}
\phi_{n}(\tau)= & \sigma_{\varepsilon}^{2} \sup _{j \geq 0} \max _{0 \leq i \leq n-1} \mathbb{E}\left|\frac{1}{n h} \sum_{t=1}^{n-i} D_{t} D_{t+i} k_{t}(\tau) k_{t+i}(\tau) \sigma_{t} \sigma_{t+i}\left(\varepsilon_{t-j}^{2}-1\right)\right|, \\
\beta_{i}= & \sum_{j=0}^{\infty}\left|\psi_{j}\right|\left|\psi_{j+i}\right|, \\
\eta_{n}(\tau)= & \sigma_{\varepsilon}^{2} \sup _{j, m \geq 0} \max _{0 \leq i \leq n-1} \mathbb{E} \mid \frac{1}{\sqrt{n h}} \mathbb{1}(m \neq j+i) \sum_{t=1}^{n-i} D_{t} D_{t+i} k_{t}(\tau) k_{t+i}(\tau) \\
& \times \sigma_{t} \sigma_{t+i} \varepsilon_{t-j} \varepsilon_{t+i-m}\left|\sum_{j=0}^{\infty} \sum_{m=0}^{\infty}\right| \psi_{j}|| \psi_{m} \mid
\end{aligned}
$$

First note that

$$
\sum_{i=0}^{\infty} \beta_{i} \leq \sum_{i=0}^{\infty} \sum_{j=0}^{\infty}\left|\psi_{j}\right|\left|\psi_{j+i}\right| \leq\left(\sum_{j=0}^{\infty}\left|\psi_{j}\right|\right)^{2}<\infty
$$

Next, let $w_{t}=\varepsilon_{t}^{2}-1$. By Assumption 2.3, $\mathbb{E}\left|w_{t}\right|^{p} \leq \mathbb{E}\left|\varepsilon_{t}\right|^{2 p}<\infty$ for some $p>1$, which implies that $w_{t}$ is uniformly integrable and for every $\epsilon>0$, there exists a $\lambda_{\epsilon}>0$ such that $\sup _{t} \mathbb{E}\left|w_{t}\right| 1\left(\left|w_{t}\right|>\lambda_{\epsilon}\right)<\epsilon$. Then define $w_{1, t}=w_{t} 1\left(\left|w_{t}\right| \leq \lambda_{\epsilon}\right)$ and $w_{2, t}=w_{t}-w_{1, t}=w_{t} 1\left(\left|w_{t}\right|>\lambda_{\epsilon}\right)$. 
As in Hall and Heyde (1980, Proof of Theorem 2.22), by the MarcinkiewiczZygmund inequality we have that

$$
\begin{aligned}
\mathbb{E} \mid & \frac{1}{n h} \sum_{t=1}^{n-i} D_{t} D_{t+i} k_{t} k_{t+i} \sigma_{t} \sigma_{t+i} w_{t} \mid \\
\leq & C \frac{1}{n h}\left(\sum_{t=1}^{n-i} k_{t}^{2}(\tau) k_{t+i}^{2}(\tau) \sigma_{t}^{2} \sigma_{t+i}^{2} \mathbb{E} w_{t-j}^{2}\right)^{1 / 2} \\
\leq & C_{1}\left[\frac{1}{n h}\left(\sum_{t=1}^{n-i} k_{t}^{2} k_{t+i}^{2} \sigma_{t}^{2} \sigma_{t+i}^{2} \mathbb{E} w_{1, t-j}^{2}\right)^{1 / 2}\right. \\
& \left.+\frac{1}{n h}\left(\sum_{t=1}^{n-i} k_{t}^{2}(\tau) k_{t+i}^{2}(\tau) \sigma_{t}^{2} \sigma_{t+i}^{2} \mathbb{E} w_{2, t-j}^{2}\right)^{1 / 2}\right] \\
= & C_{1}\left(\phi_{n, 1}(\tau)+\phi_{n, 2}(\tau)\right) .
\end{aligned}
$$

As $\left|w_{1, t-j}\right| \leq \lambda_{\epsilon}, \sup _{\tau \in[0,1]} \sigma(\tau)^{2}<\infty$, it follows from the stationarity of $w_{1, t}$ and Lemma 2.2 that

$$
\sup _{\tau \in[\delta, 1-\delta]} \phi_{n, 1}(\tau) \leq \frac{1}{n h}\left(\sup _{\tau \in[0,1]} \sigma(\tau)^{4} \mathbb{E} w_{1, t}^{2} \sup _{\tau \in[\delta, 1-\delta]} \sum_{t=1}^{n-i} k_{t}^{2}(\tau) k_{t+i}^{2}(\tau)\right)^{1 / 2} \leq \frac{C}{\sqrt{n h}} \lambda_{\epsilon} .
$$

Furthermore we get that

$$
\sup _{\tau \in[\delta, 1-\delta]} \phi_{n, 2}(\tau) \leq \frac{1}{n h} \sup _{\tau \in[0,1]} \sigma(\tau)^{2} \sup _{\tau \in[\delta, 1-\delta]} \sum_{t=1}^{n-i} k_{t}(\tau) k_{t+i}(\tau) \mathbb{E}\left|w_{2, t-j}\right| \leq C_{3} \epsilon
$$

from which it follows that $\sup _{\tau \in[\delta, 1-\delta]} \phi_{n}(\tau) \leq C\left[(n h)^{-1 / 2} \lambda_{\epsilon}+\epsilon\right]=o(1)+C \epsilon$. As we can make $\epsilon$ arbitrarily small, it follows that $\phi_{n}=\sup _{\tau \in[\delta, 1-\delta]} \phi_{n}(\tau) \rightarrow 0$ as $n \rightarrow \infty$ for all $\tau \in(0,1)$. Next we look at $\eta_{n}(\tau)$. First note that

$$
\sum_{j=0}^{\infty} \sum_{m=0}^{\infty}\left|\psi_{j}\right|\left|\psi_{m}\right|=\left(\sum_{j=0}^{\infty}\left|\psi_{j}\right|\right)^{2}<\infty
$$


Next, note that by Jensen's inequality

$$
\begin{aligned}
\mathbb{E} & \left|\frac{1}{\sqrt{n h}} \mathbb{1}(m \neq j+i) \sum_{t=1}^{n-i} D_{t} D_{t+i} k_{t}(\tau) k_{t+i}(\tau) \sigma_{t} \sigma_{t+i} \varepsilon_{t-j} \varepsilon_{t+i-m}\right| \\
\leq & \left.\frac{1}{\sqrt{n h}} \mathbb{1}_{m \neq j+i}\left[\mathbb{E}\left(\sum_{t=1}^{n-i} D_{t} D_{t+i} k_{t}(\tau) k_{t+i}(\tau) \sigma_{t} \sigma_{t+i} \varepsilon_{t-j} \varepsilon_{t+i-m}\right)\right]^{2}\right]^{1 / 2} \\
\leq & \frac{1}{\sqrt{n h}} \mathbb{1}_{m \neq j+i}\left[\sum_{s=1}^{n-i} \sum_{t=1}^{n-i} k_{s}(\tau) k_{t}(\tau) k_{s+i}(\tau) k_{t+i}(\tau) \sigma_{s} \sigma_{t} \sigma_{s+i} \sigma_{t+i}\right. \\
& \left.\mathbb{E} \varepsilon_{s-j} \varepsilon_{t-j} \varepsilon_{s+i-m} \varepsilon_{t+i-m}\right]^{1 / 2} .
\end{aligned}
$$

As $\mathbb{1}(m \neq j+i) \mathbb{E} \varepsilon_{s-j} \varepsilon_{t-j} \varepsilon_{s+i-m} \varepsilon_{t+i-m}$ can only be non-zero if $t=s$, we can deduce that

$$
\begin{aligned}
& \sup _{\tau \in[\delta, 1-\delta]} \mathbb{E}\left|\frac{1}{\sqrt{n h}} \mathbb{1}(m \neq j+i) \sum_{t=1}^{n-i} D_{t} D_{t+i} k_{t}(\tau) k_{t+i}(\tau) \sigma_{t} \sigma_{t+i} \varepsilon_{t-j} \varepsilon_{t+i-m}\right| \\
& \leq \frac{1}{\sqrt{n h}}\left[\sup _{\tau \in[\delta, 1-\delta]} \sum_{t=1}^{n-i} k_{t}^{2}(\tau) k_{t+i}^{2}(\tau) \sigma_{t}^{2} \sigma_{t+i}^{2}\right]^{1 / 2} \leq \frac{1}{\sqrt{n h}}\left[C n h \sup _{\tau \in[0,1]} \sigma(\tau)^{4}\right]^{1 / 2} \leq C .
\end{aligned}
$$

Let $\eta_{n}=\sup _{\tau \in[\delta, 1-\delta]} \eta_{n}(\tau)$, then the above shows that $\lim \sup _{n \rightarrow \infty} \eta_{n}<\infty$.

Proof of Lemma 2.8. Define the "difference" operator $\Delta_{\tau_{1}, \tau_{2}}$ applied to any function $f(\cdot)$ as

$$
\Delta_{\tau_{1}, \tau_{2}} f\left(\tau_{0}\right)=f\left(\tau_{0}+\tau_{1} h\right)-f\left(\tau_{0}+\tau_{2} h\right)
$$

Let $A_{n}\left(\tau_{0}, \tau_{1}, \tau_{2}\right)=\frac{1}{\sqrt{n h}} \sum_{t=1}^{n} \Delta_{\tau_{1}, \tau_{2}} k_{t}\left(\tau_{0}\right)\left[f(t / n)-q_{n}(\tau)\right] X_{t}$. By the Markov inequality, for any $\epsilon>0$

$$
\mathbb{P}\left[\frac{\left|A_{n}\left(\tau_{0}, \tau_{1}, \tau_{2}\right)\right|}{\left|\tau_{1}-\tau_{2}\right|} \leq \epsilon\right] \leq \epsilon^{-2} \frac{\mathbb{E} A_{n}\left(\tau_{0}, \tau_{1}, \tau_{2}\right)^{2}}{\left(\tau_{1}-\tau_{2}\right)^{2}} .
$$

We will now show that, for large enough $n,(2.28)$ can be bounded by a constant, which is sufficient for the result to hold. 
Note that

$$
\begin{aligned}
\mathbb{E} & A_{n}\left(\tau_{0}, \tau_{1}, \tau_{2}\right)^{2} \\
& =\frac{1}{n h} \sum_{i=-n+1}^{n-1} \sum_{t=1}^{n} \Delta_{\tau_{1}, \tau_{2}} k_{t}\left(\tau_{0}\right) \Delta_{\tau_{1}, \tau_{2}} k_{t+i}\left(\tau_{0}\right) \mathbb{E} X_{t} X_{t+|i|} \\
& \leq \frac{1}{n h} \sum_{i=-n+1}^{n-1} \sup _{1 \leq t \leq n-i}\left|\mathbb{E} X_{t} X_{t+|i|}\right| \sum_{t=1}^{n}\left|\Delta_{\tau_{1}, \tau_{2}} k_{t}\left(\tau_{0}\right)\right|\left|\Delta_{\tau_{1}, \tau_{2}} k_{t+i}\left(\tau_{0}\right)\right|
\end{aligned}
$$

By the compact support of the kernel, there a $C_{K}>0$ such that $K(x)=0$ for all $|x|>C_{K}$. Without loss of generality we henceforth assume that $C_{K}=$ 1. Then note that $k_{t}\left(\tau_{0}+\tau_{1} h\right)>0$ only if $\left|t / n-\tau_{0}-\tau_{1} h\right|<h$. As $\{t$ : $\left.\left|t / n-\tau_{0}-\tau_{1} h\right|<h\right\} \subseteq\left\{t:\left|t / n-\tau_{0}\right|<2 h\right\}$, this implies that $\sum_{t=1}^{n} k_{t}\left(\tau_{0}+\tau_{1} h\right)=$ $\sum_{t=n\left(\tau_{0}-2 h\right)}^{n\left(\tau_{0}+2 h\right)} k_{t}\left(\tau_{0}+\tau_{1} h\right)$. Furthermore, the Lipschitz property of the kernel implies that $\left|\Delta_{\tau_{1}, \tau_{2}} k_{t}\left(\tau_{0}\right)\right| \leq C\left|\tau_{1}-\tau_{2}\right|$. We then find that

$$
\begin{aligned}
\mathbb{E} A_{n}\left(\tau_{0}, \tau_{1}, \tau_{2}\right)^{2} & \leq \sum_{i=-n+1}^{n-1} \sup _{1 \leq t \leq n-i}\left|\mathbb{E} X_{t} X_{t+|i|}\right| \frac{1}{n h} \sum_{t=n\left(\tau_{0}-2 h\right)}^{n\left(\tau_{0}+2 h\right)} C\left(\tau_{1}-\tau_{2}\right)^{2} \\
& \leq C_{1}\left(\tau_{1}-\tau_{2}\right)^{2} \sum_{i=-n+1}^{n-1} \sup _{1 \leq t \leq n-i}\left|\mathbb{E} X_{t} X_{t+|i|}\right| \leq C_{2}\left(\tau_{1}-\tau_{2}\right)^{2}
\end{aligned}
$$

such that $(2.28)$ can be bounded by a constant not depending on $\tau_{1}$ or $\tau_{2}$, which concludes the proof.

Proof of Lemma 2.9. Let

$$
A_{n}^{*}\left(\tau_{0}, \tau_{1}, \tau_{2}\right)=\frac{1}{\sqrt{n h}} \sum_{t=1}^{n} \Delta_{\tau_{1}, \tau_{2}} k_{t}\left(\tau_{0}\right)\left[f(t / n)-q_{n}(\tau)\right] X_{t}^{*},
$$

with $\Delta_{\tau_{1}, \tau_{2}}$ defined in (2.27). Apply the Markov inequality twice, such that for any $\epsilon, \eta>0$,

$$
\begin{gathered}
\mathbb{P}\left\{\mathbb{P}^{*}\left[\frac{\left|A_{n}^{*}\left(\tau_{0}, \tau_{1}, \tau_{2}\right)\right|}{\left|f(\tau)-q_{n}(\tau)\right|\left|\tau_{1}-\tau_{2}\right|}>\epsilon\right]>\eta\right\} \\
\leq \epsilon^{-2} \eta^{-1} \frac{\mathbb{E} \mathbb{E}^{*} A_{n}^{*}\left(\tau_{0}, \tau_{1}, \tau_{2}\right)^{2}}{\left(\tau_{1}-\tau_{2}\right)^{2}\left(f(\tau)-q_{n}(\tau)\right)^{2}} .
\end{gathered}
$$

We again show that, for large enough $n$, that (2.29) can be bounded by a constant, which is sufficient for the result to hold. 
By the compact support and the Lipschitz property of the kernel, we then find that

$$
\begin{aligned}
\mathbb{E} & \mathbb{E}^{*} A_{n}^{*}\left(\tau_{0}, \tau_{1}, \tau_{2}\right)^{2} \\
& =\frac{1}{n h} \sum_{i=-n+1}^{n-1} \sum_{t=1}^{n} \Delta_{\tau_{1}, \tau_{2}} k_{t}\left(\tau_{0}\right) \Delta_{\tau_{1}, \tau_{2}} k_{t+i}\left(\tau_{0}\right) \mathbb{E}^{*} X_{t}^{*} X_{t+|i|}^{*} \\
& \leq \frac{1}{n h} \sum_{i=-n+1}^{n-1} \sup _{1 \leq t \leq n-i}\left|\mathbb{E} \mathbb{E}^{*} X_{t}^{*} X_{t+|i|}^{*}\right| \sum_{t=1}^{n}\left|\Delta_{\tau_{1}, \tau_{2}} k_{t}\left(\tau_{0}\right)\right|\left|\Delta_{\tau_{1}, \tau_{2}} k_{t+i}\left(\tau_{0}\right)\right| \\
& \leq C\left(\tau_{1}-\tau_{2}\right)^{2} \sum_{i=-n+1}^{n-1} \sup _{1 \leq t \leq n-i}\left|\mathbb{E} \mathbb{E}^{*} X_{t}^{*} X_{t+|i|}^{*}\right| \\
& \leq C_{1}\left(\tau_{1}-\tau_{2}\right)^{2}
\end{aligned}
$$

Proof of Lemma 2.10. Using the terms defined in (2.18), we define

$$
R_{n, 1}(\tau)=I_{n}(\tau)+I I_{n}(\tau)
$$

such that $R_{n}(\tau)=R_{n, 1}(\tau)+I I I_{n}(\tau)$. With $\Delta_{\tau_{1}, \tau_{2}}$ as defined in (2.27), it follows directly from the proof of Lemma 2.6 that

$$
\sup _{\tau_{1}, \tau_{2} \in[-1,1]}\left|\Delta_{\tau_{1}, \tau_{2}} I I I_{n}\left(\tau_{0}\right)\right| \leq 2 \sup _{\tau \in[\delta, 1-\delta]}\left|I I I_{n}(\tau)\right|=o(1)
$$

For $R_{n, 1}(\tau)$, as Lemmas 2.6 establishes pointwise convergence, we only need to establish stochastic equicontinuity to have uniform convergence as well. For this purpose we show that there exists some $N>0$ such that for all $n>N$, there exists some random variable $B_{n}=O_{p}(1)$ such that

$$
\left|\Delta_{\tau_{1}, \tau_{2}} R_{n, 1}\left(\tau_{0}\right)\right| \leq B_{n}\left|\tau_{1}-\tau_{2}\right|, \quad \forall \tau_{1}, \tau_{2} \in[\delta, 1-\delta],
$$

which, as shown by Andrews (1992, Lemma 1) and Davidson (2002, Thm 21.10), implies stochastic equicontinuity. To establish (2.30), we establish this bound for each term in $R_{n, 1}(\tau)$. In the following, we use generic random variables $B_{n}^{\prime}$ and $B_{n}^{\prime \prime}$, which can change each line, but are always $O_{p}(1)$ and do not depend on $\tau_{1}$ and $\tau_{2}$. First, for $I_{n}(\tau)=\left[\hat{p}(\tau)^{-1}-p(\tau)^{-1}\right] Z_{n, U}(\tau)$, a straightforward but tedious calculation shows that

$$
\left|\Delta_{\tau_{1}, \tau_{2}} I_{n}\left(\tau_{0}\right)\right| \leq B_{n}^{\prime}\left|\Delta_{\tau_{1}, \tau_{2}} Z_{n, U}\left(\tau_{0}\right)\right|+B_{n}^{\prime \prime}\left|\Delta_{\tau_{1}, \tau_{2}}\left[\hat{p}\left(\tau_{0}\right)-p\left(\tau_{0}\right)\right]\right|
$$


By taking $X_{t}=D_{t} z_{t}$, Lemmas 2.1 and 2.8 directly imply that $\left|\Delta_{\tau_{1}, \tau_{2}} Z_{n, U}\left(\tau_{0}\right)\right| \leq$ $B_{n}^{\prime}\left|\tau_{1}-\tau_{2}\right|$. Furthermore, for the second term we can write

$$
\begin{aligned}
\left|\Delta_{\tau_{1}, \tau_{2}}\left[\hat{p}\left(\tau_{0}\right)-p\left(\tau_{0}\right)\right]\right| \leq & \left|\Delta_{\tau_{1}, \tau_{2}}\left[\hat{p}\left(\tau_{0}\right)-\bar{p}\left(\tau_{0}\right)\right]\right| \\
& +\left|\Delta_{\tau_{1}, \tau_{2}}\left[\bar{p}\left(\tau_{0}\right)-p\left(\tau_{0}\right)-h^{2} \frac{\mu_{2}}{2} p^{(2)}\left(\tau_{0}\right)\right]\right| \\
& +h^{2} \frac{\mu_{2}}{2}\left|\Delta_{\tau_{1}, \tau_{2}} p^{(2)}\left(\tau_{0}\right)\right|
\end{aligned}
$$

where $\bar{p}(\tau)=\frac{1}{n h} \sum_{t=1}^{n} k_{t}(\tau) p\left(\frac{t}{n}\right)$. For the first part we can use Lemma 2.8 with $X_{t}=D_{t}-p\left(\frac{t}{n}\right)$ to establish the bound $\left|\Delta_{\tau_{1}, \tau_{2}}\left[\hat{p}\left(\tau_{0}\right)-\bar{p}\left(\tau_{0}\right)\right]\right| \leq B_{n}^{\prime}\left|\tau_{1}-\tau_{2}\right| / \sqrt{n h}$, while for the second part we have uniform convergence by Lemma 2.3(i), which implies stochastic equicontinuity, as

$$
\begin{aligned}
& \left|\Delta_{\tau_{1}, \tau_{2}}\left[\bar{p}\left(\tau_{0}\right)-p\left(\tau_{0}\right)-h^{2} \frac{\mu_{2}}{2} p^{(2)}\left(\tau_{0}\right)\right]\right| \\
& \quad \leq 2 \sup _{\tau \in[\delta, 1-\delta]}\left|\bar{p}\left(\tau_{0}\right)-p\left(\tau_{0}\right)-h^{2} \frac{\mu_{2}}{2} p^{(2)}\left(\tau_{0}\right)\right| \\
& \quad \leq C \max \left\{h^{3}, \frac{1}{n h}\right\},
\end{aligned}
$$

and for any $\tau_{1}, \tau_{2}$, we can find an $n$ such that $\max \left\{h^{3}, \frac{1}{n h}\right\}$ is smaller than $\left|\tau_{1}-\tau_{2}\right| / \sqrt{n h}$. Finally, for the third term we get, by the smoothness of $p^{(2)}(\cdot)$, that $\left|\Delta_{\tau_{1}, \tau_{2}} p^{(2)}\left(\tau_{0}\right)\right| \leq C h\left|\tau_{1}-\tau_{2}\right|$. Combining these three results, it therefore follows that

$$
\left|\Delta_{\tau_{1}, \tau_{2}}\left[\hat{p}\left(\tau_{0}\right)-p\left(\tau_{0}\right)\right]\right| \leq B_{n}^{\prime}\left|\tau_{1}-\tau_{2}\right| / \sqrt{n h} .
$$

Similarly, for $I I_{n, 1}(\tau)$, Lemma 2.8 with $X_{t}=\left[m\left(\tau_{0}\right)-p^{-1} \bar{m}_{p}\left(\tau_{0}\right)\right]\left[D_{t}-p\left(\frac{t}{n}\right)\right]$ provides the appropriate bound, as $\left|\mathbb{E} X_{t} X_{t+i}\right| \leq C \mathbb{C o v}\left(D_{t}, D_{t+i}\right)$. For $I I_{n, 2}(\tau)$, we have that

$$
\begin{aligned}
& \left|\Delta_{\tau_{1}, \tau_{2}} I I_{n, 2}\left(\tau_{0}\right)\right| \leq B_{n}^{\prime} \sqrt{n h} \Delta_{\tau_{1}, \tau_{2}}\left[\hat{p}\left(\tau_{0}\right)-p\left(\tau_{0}\right)\right]^{2} \\
& \quad+B_{n}^{\prime \prime} \sup _{\tau \in[-1,1]}[\hat{p}(\tau)-p(\tau)]^{2} \Delta_{\tau_{1}, \tau_{2}} \bar{m}_{p}\left(\tau_{0}\right) .
\end{aligned}
$$

By (2.31), we have that $\sqrt{n h} \Delta_{\tau_{1}, \tau_{2}}\left[\hat{p}\left(\tau_{0}\right)-p\left(\tau_{0}\right)\right]^{2} \leq B_{n}^{\prime}\left|\tau_{1}-\tau_{2}\right|$, while, again by (2.31) and pointwise convergence,

$$
\sqrt{n h} \sup _{\tau \in[-1,1]}[\hat{p}(\tau)-p(\tau)]^{2}=O_{p}\left(\max \left\{\sqrt{n h^{9}}, \frac{1}{\sqrt{n h}}\right\}\right),
$$


while it follows from the Lipschitz properties that $\Delta_{\tau_{1}, \tau_{2}} \bar{m}_{p}\left(\tau_{0}\right) \leq C\left|\tau_{1}-\tau_{2}\right|$. Finally, the same arguments used above can also be used to establish that

$$
\left|\Delta_{\tau_{1}, \tau_{2}} I I_{n, 3}\left(\tau_{0}\right)\right| \leq B_{n}^{\prime}\left|\tau_{1}-\tau_{2}\right|
$$

We treat the bootstrap terms in exactly the same way using the decomposition in (2.22). Again, uniform convergence of $I I I_{n}^{*}(\tau)$ is immediate from the proof of Lemma 2.7. For the other terms $R_{n, 1}^{*}(\tau)=R_{n}^{*}(\tau)-I I I_{n}^{*}(\tau)$, we again establish stochastic equicontinuity, although now conditionally, that is we show that

$$
\left|\Delta_{\tau_{1}, \tau_{2}} R_{n, 1}^{*}\left(\tau_{0}\right)\right| \leq B_{n}^{*}\left|\tau_{1}-\tau_{2}\right|, \quad \forall \tau_{1}, \tau_{2} \in[\delta, 1-\delta]
$$

where $B_{n}^{*}=O_{p}^{*}(1)$

As for $I_{n}(\tau)$, we can bound $I_{n, 1}^{*}(\tau)$ as

$$
\left|\Delta_{\tau_{1}, \tau_{2}} I_{n, 1}^{*}\left(\tau_{0}\right)\right| \leq B_{n}^{\prime}\left|\Delta_{\tau_{1}, \tau_{2}} Z_{n, U}\left(\tau_{0}\right)\right|+B_{n}^{* \prime \prime}\left|\Delta_{\tau_{1}, \tau_{2}}\left[\hat{p}\left(\tau_{0}\right)-p\left(\tau_{0}\right)\right]\right|
$$

The second part can be bounded using (2.31), while for the first, we apply Lemma 2.9 with $X_{t}^{*}=D_{t} z_{t} \xi_{t}^{*}$. First we show the summability of the bootstrap covariances:

$$
\sum_{i=0}^{n-1} i \sup _{t}\left|\mathbb{E} \mathbb{E}^{*} D_{t} z_{t} \xi_{t}^{*} D_{t+i} z_{t+i} \xi_{t+i}^{*}\right| \leq \sum_{i=0}^{n-1} i R_{U}(i) \gamma^{i} \leq \sum_{i=0}^{n-1} i R_{U}(i)<\infty
$$

The bound on $\Delta_{\tau_{1}, \tau_{2}} I_{n, 1}\left(\tau_{0}\right)$ now follows directly from Lemma 2.9 .

Similarly, for $I_{n, 2}^{*}(\tau)$, take $X_{t}^{*}=\left[m\left(\frac{t}{n}\right)-\tilde{m}\left(\frac{t}{n}\right)\right] D_{t} \xi_{t}^{*}$ and note that, by $(2.23)$

$$
\begin{aligned}
& \sum_{i=0}^{n-1} i \sup _{t}\left|\mathbb{E} \mathbb{E}^{*}\left[m\left(\frac{t}{n}\right)-\tilde{m}\left(\frac{t}{n}\right)\right] D_{t} \xi_{t}^{*}\left[m\left(\frac{t+i}{n}\right)-\tilde{m}\left(\frac{t+i}{n}\right)\right] D_{t+i} \xi_{t+i}^{*}\right| \\
& \leq \sum_{i=0}^{n-1} i \gamma^{i} \sup _{\tau \in[\delta, 1-\delta]}\|m(\tau)-\tilde{m}(\tau)\|^{2} \leq C \ell \max \left\{\tilde{h}^{4}, \frac{1}{n \tilde{h}}\right\}<C_{1},
\end{aligned}
$$

such that the bound follows directly from Lemma 2.9.

The remaining terms do not contain bootstrap quantities, and can be treated entirely analogously to their non-bootstrap counterparts to show that (2.29) holds. 


\section{C Additional simulation results}

We present here additional simulation results that are part of the specifications mentioned in Section 5.1. Specifically, we first show the homoskedastic results. Second, we present additional tables for heteroskedastic DGPs that vary the parameters $k$ and $a$. They define the volatility process given in equation (2.13) above. Third, there are more results with missing data. We only present results for one bandwidth $h$ in the chapter. Here, the other bandwidths are considered. We decide to leave out these tables in the core part of the chapter, because they are very similar and the main messages are the same. The results in this Appendix are obtained using 1000 Monte Carlo repetitions and $B=599$. This differs from the results presented above (where we used $5000 \mathrm{MC}$ and 999 bootstrap repetitions) due to computational constraints.

\section{C.1 Homoskedastic DGPs}




\begin{tabular}{|c|c|c|c|c|c|}
\hline \multirow[b]{2}{*}{$h$} & \multirow[b]{2}{*}{ DGP } & \multirow{2}{*}{$\frac{-}{\text { SWB }}$} & \multirow{2}{*}{$\begin{array}{c}\gamma=0 \\
\mathrm{WB}\end{array}$} & \multicolumn{2}{|c|}{$\gamma=0.2$} \\
\hline & & & & AWB & DWB \\
\hline \multirow{5}{*}{0.02} & 0 & $0.906(0.500)$ & $0.948(0.519)$ & $0.953(0.512)$ & $0.948(0.522)$ \\
\hline & $A R_{0.2}$ & $0.848(0.480)$ & $0.904(0.505)$ & $0.919(0.509)$ & $0.905(0.507)$ \\
\hline & $A R_{0.5}$ & $0.675(0.375)$ & $0.790(0.430)$ & $0.826(0.451)$ & $0.789(0.430)$ \\
\hline & $A R_{-0.5}$ & $0.949(0.385)$ & $0.990(0.449)$ & $0.989(0.416)$ & $0.990(0.448)$ \\
\hline & $M A_{0.2}$ & $0.859(0.481)$ & $0.914(0.505)$ & $0.927(0.510)$ & $0.915(0.507)$ \\
\hline \multirow{5}{*}{0.04} & 0 & $0.911(0.372)$ & $0.955(0.400)$ & $0.956(0.401)$ & $0.955(0.401)$ \\
\hline & $A R_{0.2}$ & $0.849(0.356)$ & $0.908(0.390)$ & $0.919(0.401)$ & $0.909(0.391)$ \\
\hline & $A R_{0.5}$ & $0.667(0.279)$ & $0.791(0.341)$ & $0.825(0.368)$ & $0.792(0.340)$ \\
\hline & $A R_{-0.5}$ & $0.964(0.288)$ & $0.996(0.355)$ & $0.995(0.332)$ & $0.996(0.353)$ \\
\hline & $M A_{0.2}$ & $0.862(0.356)$ & $0.921(0.391)$ & $0.929(0.402)$ & $0.921(0.392)$ \\
\hline \multirow{5}{*}{0.06} & 0 & $919(0.310)$ & $0.963(0.345)$ & $0.962(0.351)$ & $0.961(0.345)$ \\
\hline & $A R_{0.2}$ & $0.853(0.297)$ & $0.918(0.336)$ & $0.929(0.353)$ & $0.919(0.338)$ \\
\hline & $A R_{0.5}$ & $0.663(0.233)$ & $0.810(0.300)$ & $0.840(0.328)$ & $0.808(0.299)$ \\
\hline & $A R_{-0.5}$ & $0.969(0.240)$ & $0.998(0.311)$ & $0.997(0.297)$ & $0.998(0.310)$ \\
\hline & $M A_{0.2}$ & $0.869(0.297)$ & $0.930(0.338)$ & $0.937(0.354)$ & $0.930(0.338)$ \\
\hline \multirow[b]{2}{*}{$h$} & \multirow[b]{2}{*}{ DGP } & \multicolumn{2}{|c|}{$\gamma=0.4$} & \multicolumn{2}{|c|}{$\gamma=0.6$} \\
\hline & & AWB & DWB & AWB & DWB \\
\hline \multirow{5}{*}{0.02} & 0 & $0.944(0.474)$ & $0.948(0.523)$ & $0.906(0.403)$ & $0.949(0.522)$ \\
\hline & $A R_{0.2}$ & $0.913(0.482)$ & $0.906(0.505)$ & $0.873(0.423)$ & $0.904(0.506)$ \\
\hline & $A R_{0.5}$ & $0.832(0.449)$ & $0.787(0.429)$ & $0.797(0.414)$ & $0.786(0.431)$ \\
\hline & $A R_{-0.5}$ & $0.982(0.366)$ & $0.989(0.447)$ & $0.961(0.296)$ & $0.989(0.449)$ \\
\hline & $M A_{0.2}$ & $0.920(0.484)$ & $0.913(0.507)$ & $0.881(0.421)$ & $0.914(0.508)$ \\
\hline \multirow{5}{*}{0.04} & 0 & $0.947(0.382)$ & $0.955(0.402)$ & $0.916(0.339)$ & $0.956(0.401)$ \\
\hline & $A R_{0.2}$ & $0.914(0.393)$ & $0.910(0.390)$ & $0.883(0.362)$ & $0.909(0.391)$ \\
\hline & $A R_{0.5}$ & $0.834(0.380)$ & $0.787(0.340)$ & $0.813(0.365)$ & $0.787(0.340)$ \\
\hline & $A R_{-0.5}$ & $0.990(0.299)$ & $0.996(0.353)$ & $0.973(0.259)$ & $0.996(0.354)$ \\
\hline & $M A_{0.2}$ & $0.922(0.393)$ & $0.920(0.392)$ & $0.892(0.357)$ & $0.921(0.391)$ \\
\hline \multirow{5}{*}{0.06} & 0 & $0.955(0.343)$ & $0.962(0.346)$ & $0.932(0.318)$ & $0.962(0.345)$ \\
\hline & $A R_{0.2}$ & $0.924(0.354)$ & $0.919(0.337)$ & $0.903(0.338)$ & $0.919(0.338)$ \\
\hline & $A R_{0.5}$ & $0.852(0.346)$ & $0.804(0.299)$ & $0.842(0.343)$ & $0.805(0.299)$ \\
\hline & $A R_{-0.5}$ & $0.993(0.279)$ & $0.998(0.310)$ & $0.983(0.263)$ & $0.998(0.310)$ \\
\hline & $M A_{0.2}$ & $0.934(0.353)$ & $0.929(0.339)$ & $0.910(0.333)$ & $0.930(0.338)$ \\
\hline
\end{tabular}

Table 2.6: Pointwise coverage probabilities 


\begin{tabular}{|c|c|c|c|c|c|}
\hline \multirow[b]{2}{*}{$h$} & \multirow[b]{2}{*}{ DGP } & \multirow{2}{*}{$\frac{-}{\text { SWB }}$} & \multirow{2}{*}{$\begin{array}{c}\gamma=0 \\
\mathrm{WB}\end{array}$} & \multicolumn{2}{|c|}{$\gamma=0.2$} \\
\hline & & & & AWB & DWB \\
\hline \multirow{5}{*}{0.02} & 0 & $0.846(0.462)$ & $0.890(0.479)$ & $0.883(0.473)$ & $0.881(0.481)$ \\
\hline & $A R_{0.2}$ & $0.748(0.443)$ & $0.814(0.467)$ & $0.844(0.469)$ & $0.829(0.468)$ \\
\hline & $A R_{0.5}$ & $0.400(0.346)$ & $0.634(0.397)$ & $0.693(0.416)$ & $0.661(0.397)$ \\
\hline & $A R_{-0.5}$ & $0.982(0.355)$ & $0.984(0.415)$ & $0.983(0.384)$ & $0.986(0.413)$ \\
\hline & $M A_{0.2}$ & $0.754(0.444)$ & $0.828(0.466)$ & $0.847(0.470)$ & $0.815(0.468)$ \\
\hline \multirow{5}{*}{0.04} & 0 & $0.880(0.345)$ & $0.907(0.370)$ & $0.904(0.371)$ & $0.899(0.372)$ \\
\hline & $A R_{0.2}$ & $0.731(0.330)$ & $0.801(0.361)$ & $0.845(0.371)$ & $0.831(0.362)$ \\
\hline & $A R_{0.5}$ & $0.367(0.259)$ & $0.589(0.316)$ & $0.661(0.340)$ & $0.598(0.315)$ \\
\hline & $A R_{-0.5}$ & $0.990(0.267)$ & $0.995(0.329)$ & $0.996(0.308)$ & $0.997(0.327)$ \\
\hline & $M A_{0.2}$ & $0.755(0.330)$ & $0.817(0.362)$ & $0.838(0.373)$ & $0.824(0.363)$ \\
\hline \multirow{5}{*}{0.06} & 0 & $887(0.288)$ & $0.915(0.320)$ & $0.904(0.326)$ & $0.909(0.321)$ \\
\hline & $A R_{0.2}$ & $0.753(0.275)$ & $0.807(0.312)$ & $0.828(0.327)$ & $0.817(0.314)$ \\
\hline & $A R_{0.5}$ & $0.368(0.216)$ & $0.590(0.278)$ & $0.648(0.304)$ & $0.592(0.277)$ \\
\hline & $A R_{-0.5}$ & $0.995(0.223)$ & $0.999(0.288)$ & $0.998(0.275)$ & $0.998(0.287)$ \\
\hline & $M A_{0.2}$ & $0.779(0.276)$ & $0.840(0.313)$ & $0.851(0.328)$ & $0.839(0.314)$ \\
\hline \multirow[b]{2}{*}{$h$} & \multirow[b]{2}{*}{ DGP } & \multicolumn{2}{|c|}{$\gamma=0.4$} & \multicolumn{2}{|c|}{$\gamma=0.6$} \\
\hline & & AWB & DWB & AWB & DWB \\
\hline \multirow{5}{*}{0.02} & 0 & $0.873(0.437)$ & $0.919(0.482)$ & $0.768(0.371)$ & $0.906(0.482)$ \\
\hline & $A R_{0.2}$ & $0.817(0.445)$ & $0.832(0.466)$ & $0.739(0.390)$ & $0.845(0.466)$ \\
\hline & $A R_{0.5}$ & $0.676(0.414)$ & $0.644(0.396)$ & $0.623(0.381)$ & $0.643(0.398)$ \\
\hline & $A R_{-0.5}$ & $0.970(0.337)$ & $0.984(0.412)$ & $0.940(0.273)$ & $0.989(0.414)$ \\
\hline & $M A_{0.2}$ & $0.828(0.446)$ & $0.863(0.468)$ & $0.727(0.389)$ & $0.846(0.468)$ \\
\hline \multirow{5}{*}{0.04} & 0 & $0.883(0.354)$ & $0.915(0.372)$ & $0.792(0.313)$ & $0.916(0.371)$ \\
\hline & $A R_{0.2}$ & $0.796(0.364)$ & $0.820(0.361)$ & $0.742(0.335)$ & $0.826(0.362)$ \\
\hline & $A R_{0.5}$ & $0.653(0.352)$ & $0.581(0.315)$ & $0.569(0.338)$ & $0.542(0.315)$ \\
\hline & $A R_{-0.5}$ & $0.986(0.277)$ & $0.990(0.327)$ & $0.947(0.239)$ & $0.993(0.328)$ \\
\hline & $M A_{0.2}$ & $0.835(0.364)$ & $0.849(0.363)$ & $0.740(0.330)$ & $0.842(0.362)$ \\
\hline \multirow{5}{*}{0.06} & 0 & $0.903(0.318)$ & $0.926(0.321)$ & $0.833(0.295)$ & $0.936(0.320)$ \\
\hline & $A R_{0.2}$ & $0.806(0.328)$ & $0.825(0.313)$ & $0.748(0.312)$ & $0.837(0.314)$ \\
\hline & $A R_{0.5}$ & $0.677(0.321)$ & $0.595(0.277)$ & $0.586(0.318)$ & $0.553(0.278)$ \\
\hline & $A R_{-0.5}$ & $0.988(0.258)$ & $0.999(0.287)$ & $0.958(0.244)$ & $0.997(0.288)$ \\
\hline & $M A_{0.2}$ & $0.850(0.327)$ & $0.867(0.314)$ & $0.774(0.309)$ & $0.854(0.313)$ \\
\hline
\end{tabular}

Table 2.7: Simultaneous coverage probabilities over G.sub 


\begin{tabular}{|c|c|c|c|c|c|}
\hline \multirow[b]{2}{*}{$h$} & \multirow[b]{2}{*}{ DGP } & \multirow{2}{*}{$\frac{-}{\text { SWB }}$} & \multirow{2}{*}{$\begin{array}{c}\gamma=0 \\
\mathrm{WB}\end{array}$} & \multicolumn{2}{|c|}{$\gamma=0.2$} \\
\hline & & & & AWB & DWB \\
\hline \multirow{5}{*}{0.02} & 0 & $0.807(0.366)$ & $0.880(0.380)$ & $0.883(0.374)$ & $0.885(0.381)$ \\
\hline & $A R_{0.2}$ & $0.631(0.351)$ & $0.770(0.369)$ & $0.832(0.372)$ & $0.798(0.370)$ \\
\hline & $A R_{0.5}$ & $0.230(0.274)$ & $0.554(0.314)$ & $0.640(0.330)$ & $0.559(0.314)$ \\
\hline & $A R_{-0.5}$ & $0.970(0.281)$ & $0.977(0.328)$ & $0.980(0.304)$ & $0.979(0.327)$ \\
\hline & $M A_{0.2}$ & $0.654(0.351)$ & $0.804(0.369)$ & $0.832(0.373)$ & $0.804(0.370)$ \\
\hline \multirow{5}{*}{0.04} & 0 & $0.849(0.268)$ & $0.894(0.288)$ & $0.891(0.288)$ & $0.891(0.288)$ \\
\hline & $A R_{0.2}$ & $0.639(0.256)$ & $0.741(0.280)$ & $0.782(0.288)$ & $0.769(0.281)$ \\
\hline & $A R_{0.5}$ & $0.186(0.201)$ & $0.409(0.245)$ & $0.487(0.264)$ & $0.406(0.244)$ \\
\hline & $A R_{-0.5}$ & $0.996(0.207)$ & $0.993(0.255)$ & $0.995(0.239)$ & $0.994(0.254)$ \\
\hline & $M A_{0.2}$ & $0.686(0.256)$ & $0.775(0.281)$ & $0.816(0.289)$ & $0.789(0.282)$ \\
\hline \multirow{5}{*}{0.06} & 0 & $850(0.222)$ & $0.897(0.246)$ & $0.890(0.251)$ & $0.888(0.247)$ \\
\hline & $A R_{0.2}$ & $0.652(0.212)$ & $0.752(0.241)$ & $0.780(0.252)$ & $0.772(0.241)$ \\
\hline & $A R_{0.5}$ & $0.180(0.166)$ & $0.418(0.214)$ & $0.480(0.234)$ & $0.395(0.213)$ \\
\hline & $A R_{-0.5}$ & $0.995(0.172)$ & $0.998(0.222)$ & $0.998(0.212)$ & $1.000(0.221)$ \\
\hline & $M A_{0.2}$ & $0.706(0.213)$ & $0.792(0.241)$ & $0.818(0.253)$ & $0.792(0.242)$ \\
\hline \multirow[b]{2}{*}{$h$} & \multirow[b]{2}{*}{ DGP } & \multicolumn{2}{|c|}{$\gamma=0.4$} & \multicolumn{2}{|c|}{$\gamma=0.6$} \\
\hline & & AWB & DWB & AWB & DWB \\
\hline \multirow{5}{*}{0.02} & 0 & $0.868(0.346)$ & $0.901(0.382)$ & $0.756(0.294)$ & $0.895(0.381)$ \\
\hline & $A R_{0.2}$ & $0.802(0.353)$ & $0.807(0.369)$ & $0.690(0.310)$ & $0.799(0.369)$ \\
\hline & $A R_{0.5}$ & $0.596(0.329)$ & $0.532(0.313)$ & $0.536(0.304)$ & $0.534(0.315)$ \\
\hline & $A R_{-0.5}$ & $0.960(0.267)$ & $0.976(0.326)$ & $0.895(0.216)$ & $0.977(0.328)$ \\
\hline & $M A_{0.2}$ & $0.814(0.354)$ & $0.827(0.371)$ & $0.694(0.308)$ & $0.831(0.371)$ \\
\hline \multirow{5}{*}{0.04} & 0 & $0.850(0.274)$ & $0.893(0.289)$ & $0.719(0.244)$ & $0.897(0.288)$ \\
\hline & $A R_{0.2}$ & $0.744(0.283)$ & $0.762(0.280)$ & $0.623(0.261)$ & $0.763(0.281)$ \\
\hline & $A R_{0.5}$ & $0.491(0.274)$ & $0.395(0.244)$ & $0.425(0.264)$ & $0.391(0.245)$ \\
\hline & $A R_{-0.5}$ & $0.977(0.215)$ & $0.987(0.254)$ & $0.922(0.186)$ & $0.996(0.254)$ \\
\hline & $M A_{0.2}$ & $0.768(0.283)$ & $0.798(0.281)$ & $0.656(0.257)$ & $0.792(0.281)$ \\
\hline \multirow{5}{*}{0.06} & 0 & $0.872(0.245)$ & $0.911(0.247)$ & $0.774(0.228)$ & $0.909(0.247)$ \\
\hline & $A R_{0.2}$ & $0.752(0.253)$ & $0.765(0.241)$ & $0.647(0.241)$ & $0.761(0.241)$ \\
\hline & $A R_{0.5}$ & $0.503(0.248)$ & $0.402(0.214)$ & $0.428(0.246)$ & $0.398(0.214)$ \\
\hline & $A R_{-0.5}$ & $0.986(0.199)$ & $0.996(0.221)$ & $0.950(0.188)$ & $0.999(0.222)$ \\
\hline & $M A_{0.2}$ & $0.798(0.253)$ & $0.810(0.242)$ & $0.689(0.239)$ & $0.795(0.241)$ \\
\hline
\end{tabular}

Table 2.8: Simultaneous coverage probabilities over $G$ 
2.C.2 Varying $a$ and $k$ in the heteroskedastic DGPs 


\begin{tabular}{|c|c|c|c|c|c|}
\hline \multirow[b]{2}{*}{$h$} & \multirow[b]{2}{*}{ DGP } & \multirow{2}{*}{$\frac{-}{\mathrm{SWB}}$} & \multirow{2}{*}{$\begin{array}{c}\gamma=0 \\
\mathrm{WB}\end{array}$} & \multicolumn{2}{|c|}{$\gamma=0.2$} \\
\hline & & & & AWB & DWB \\
\hline \multirow{5}{*}{0.02} & 0 & $0.926(0.717)$ & $0.946(0.727)$ & $0.952(0.711)$ & $0.946(0.729)$ \\
\hline & $A R_{0.2}$ & $0.861(0.686)$ & $0.902(0.706)$ & $0.919(0.708)$ & $0.902(0.708)$ \\
\hline & $A R_{0.5}$ & $0.673(0.536)$ & $0.780(0.600)$ & $0.819(0.626)$ & $0.778(0.600)$ \\
\hline & $A R_{-0.5}$ & $0.959(0.550)$ & $0.990(0.622)$ & $0.988(0.571)$ & $0.989(0.620)$ \\
\hline & $M A_{0.2}$ & $0.871(0.687)$ & $0.911(0.708)$ & $0.925(0.709)$ & $0.911(0.708)$ \\
\hline \multirow{5}{*}{0.04} & 0 & $0.921(0.538)$ & $0.950(0.557)$ & $0.951(0.551)$ & $0.951(0.558)$ \\
\hline & $A R_{0.2}$ & $0.862(0.517)$ & $0.900(0.542)$ & $0.911(0.552)$ & $0.900(0.543)$ \\
\hline & $A R_{0.5}$ & $0.667(0.404)$ & $0.765(0.469)$ & $0.805(0.500)$ & $0.768(0.469)$ \\
\hline & $A R_{-0.5}$ & $0.970(0.415)$ & $0.996(0.486)$ & $0.993(0.450)$ & $0.995(0.483)$ \\
\hline & $M A_{0.2}$ & $0.873(0.517)$ & $0.912(0.544)$ & $0.920(0.553)$ & $0.911(0.544)$ \\
\hline \multirow{5}{*}{0.06} & 0 & $0.925(0.451)$ & $0.955(0.478)$ & $0.955(0.477)$ & $0.955(0.479)$ \\
\hline & $A R_{0.2}$ & $0.865(0.433)$ & $0.906(0.465)$ & $0.917(0.479)$ & $0.908(0.466)$ \\
\hline & $A R_{0.5}$ & $0.668(0.340)$ & $0.778(0.407)$ & $0.815(0.438)$ & $0.780(0.407)$ \\
\hline & $A R_{-0.5}$ & $0.980(0.348)$ & $0.997(0.421)$ & $0.995(0.396)$ & $0.997(0.419)$ \\
\hline & $M A_{0.2}$ & $0.878(0.433)$ & $0.919(0.466)$ & $0.925(0.480)$ & $0.918(0.467)$ \\
\hline & \multirow[b]{2}{*}{ DGP } & \multicolumn{2}{|c|}{$\gamma=0.4$} & \multicolumn{2}{|c|}{$\gamma=0.6$} \\
\hline & & AWB & DWB & AWB & DWB \\
\hline \multirow{5}{*}{0.02} & 0 & $0.944(0.653)$ & $0.945(0.729)$ & $0.906(0.551)$ & $0.947(0.730)$ \\
\hline & $A R_{0.2}$ & $0.914(0.665)$ & $0.903(0.704)$ & $0.869(0.580)$ & $0.901(0.707)$ \\
\hline & $A R_{0.5}$ & $0.829(0.614)$ & $0.779(0.596)$ & $0.791(0.560)$ & $0.777(0.599)$ \\
\hline & $A R_{-0.5}$ & $0.983(0.496)$ & $0.989(0.621)$ & $0.960(0.396)$ & $0.989(0.621)$ \\
\hline & $M A_{0.2}$ & $0.919(0.666)$ & $0.908(0.708)$ & $0.880(0.577)$ & $0.912(0.709)$ \\
\hline \multirow{5}{*}{0.04} & 0 & $0.939(0.515)$ & $0.948(0.558)$ & $0.903(0.449)$ & $0.951(0.559)$ \\
\hline & $A R_{0.2}$ & $0.904(0.530)$ & $0.900(0.540)$ & $0.865(0.476)$ & $0.897(0.542)$ \\
\hline & $A R_{0.5}$ & $0.815(0.506)$ & $0.766(0.466)$ & $0.792(0.478)$ & $0.763(0.466)$ \\
\hline & $A R_{-0.5}$ & $0.988(0.401)$ & $0.995(0.483)$ & $0.968(0.334)$ & $0.995(0.484)$ \\
\hline & $M A_{0.2}$ & $0.911(0.530)$ & $0.909(0.543)$ & $0.877(0.473)$ & $0.911(0.544)$ \\
\hline \multirow{5}{*}{0.06} & 0 & $0.942(0.454)$ & $0.952(0.478)$ & $0.912(0.406)$ & $0.954(0.478)$ \\
\hline & $A R_{0.2}$ & $0.910(0.467)$ & $0.904(0.465)$ & $0.878(0.429)$ & $0.903(0.466)$ \\
\hline & $A R_{0.5}$ & $0.825(0.450)$ & $0.775(0.405)$ & $0.813(0.437)$ & $0.775(0.404)$ \\
\hline & $A R_{-0.5}$ & $0.991(0.360)$ & $0.997(0.419)$ & $0.977(0.316)$ & $0.997(0.420)$ \\
\hline & $M A_{0.2}$ & $0.917(0.467)$ & $0.913(0.466)$ & $0.889(0.426)$ & $0.915(0.466)$ \\
\hline
\end{tabular}

Table 2.9: Pointwise coverage probabilities (average median interval length) for $k=2$ and $a=0.3$. 


\begin{tabular}{|c|c|c|c|c|c|}
\hline \multirow[b]{2}{*}{$h$} & \multirow[b]{2}{*}{ DGP } & \multirow{2}{*}{$\frac{-}{\text { SWB }}$} & \multirow{2}{*}{$\begin{array}{c}\gamma=0 \\
\mathrm{WB}\end{array}$} & \multicolumn{2}{|c|}{$\gamma=0.2$} \\
\hline & & & & AWB & DWB \\
\hline \multirow{5}{*}{0.02} & 0 & $0.881(0.662)$ & $0.905(0.671)$ & $0.914(0.656)$ & $0.912(0.673)$ \\
\hline & $A R_{0.2}$ & $0.781(0.633)$ & $0.847(0.652)$ & $0.876(0.653)$ & $0.845(0.654)$ \\
\hline & $A R_{0.5}$ & $0.409(0.495)$ & $0.659(0.554)$ & $0.718(0.577)$ & $0.641(0.554)$ \\
\hline & $A R_{-0.5}$ & $0.978(0.508)$ & $0.985(0.575)$ & $0.984(0.528)$ & $0.981(0.573)$ \\
\hline & $M A_{0.2}$ & $0.782(0.634)$ & $0.848(0.653)$ & $0.882(0.653)$ & $0.853(0.654)$ \\
\hline \multirow{5}{*}{0.04} & 0 & $0.881(0.499)$ & $0.908(0.517)$ & $0.912(0.512)$ & $0.908(0.517)$ \\
\hline & $A R_{0.2}$ & $0.757(0.479)$ & $0.817(0.503)$ & $0.857(0.512)$ & $0.836(0.503)$ \\
\hline & $A R_{0.5}$ & $0.333(0.375)$ & $0.559(0.435)$ & $0.631(0.464)$ & $0.548(0.435)$ \\
\hline & $A R_{-0.5}$ & $0.990(0.384)$ & $0.995(0.451)$ & $0.986(0.417)$ & $0.987(0.448)$ \\
\hline & $M A_{0.2}$ & $0.779(0.479)$ & $0.831(0.504)$ & $0.853(0.513)$ & $0.824(0.505)$ \\
\hline \multirow{5}{*}{0.06} & 0 & $0.883(0.419)$ & $0.921(0.444)$ & $0.924(0.443)$ & $0.922(0.445)$ \\
\hline & $A R_{0.2}$ & $0.768(0.402)$ & $0.823(0.432)$ & $0.841(0.444)$ & $0.834(0.433)$ \\
\hline & $A R_{0.5}$ & $0.351(0.316)$ & $0.536(0.378)$ & $0.617(0.407)$ & $0.551(0.377)$ \\
\hline & $A R_{-0.5}$ & $0.999(0.323)$ & $0.999(0.391)$ & $0.993(0.367)$ & $0.995(0.390)$ \\
\hline & $M A_{0.2}$ & $0.796(0.403)$ & $0.831(0.433)$ & $0.855(0.445)$ & $0.844(0.434)$ \\
\hline & \multirow[b]{2}{*}{ DGP } & \multicolumn{2}{|c|}{$\gamma=0.4$} & \multicolumn{2}{|c|}{$\gamma=0.6$} \\
\hline & & AWB & DWB & AWB & DWB \\
\hline \multirow{5}{*}{0.02} & 0 & $0.903(0.602)$ & $0.918(0.673)$ & $0.806(0.509)$ & $0.919(0.673)$ \\
\hline & $A R_{0.2}$ & $0.853(0.614)$ & $0.846(0.651)$ & $0.781(0.534)$ & $0.863(0.653)$ \\
\hline & $A R_{0.5}$ & $0.701(0.566)$ & $0.635(0.550)$ & $0.629(0.516)$ & $0.648(0.553)$ \\
\hline & $A R_{-0.5}$ & $0.962(0.459)$ & $0.975(0.574)$ & $0.901(0.365)$ & $0.980(0.573)$ \\
\hline & $M A_{0.2}$ & $0.872(0.614)$ & $0.864(0.654)$ & $0.777(0.532)$ & $0.878(0.654)$ \\
\hline \multirow{5}{*}{0.04} & 0 & $0.886(0.478)$ & $0.930(0.517)$ & $0.777(0.415)$ & $0.914(0.518)$ \\
\hline & $A R_{0.2}$ & $0.808(0.492)$ & $0.815(0.501)$ & $0.749(0.441)$ & $0.829(0.503)$ \\
\hline & $A R_{0.5}$ & $0.639(0.469)$ & $0.541(0.432)$ & $0.582(0.443)$ & $0.553(0.432)$ \\
\hline & $A R_{-0.5}$ & $0.970(0.371)$ & $0.988(0.448)$ & $0.926(0.309)$ & $0.989(0.449)$ \\
\hline & $M A_{0.2}$ & $0.838(0.492)$ & $0.862(0.504)$ & $0.723(0.438)$ & $0.828(0.505)$ \\
\hline \multirow{5}{*}{0.06} & 0 & $0.893(0.421)$ & $0.925(0.444)$ & $0.789(0.376)$ & $0.915(0.444)$ \\
\hline & $A R_{0.2}$ & $0.807(0.433)$ & $0.818(0.431)$ & $0.749(0.398)$ & $0.836(0.432)$ \\
\hline & $A R_{0.5}$ & $0.646(0.418)$ & $0.540(0.375)$ & $0.593(0.404)$ & $0.542(0.375)$ \\
\hline & $A R_{-0.5}$ & $0.982(0.335)$ & $0.997(0.389)$ & $0.952(0.292)$ & $0.998(0.390)$ \\
\hline & $M A_{0.2}$ & $0.842(0.434)$ & $0.853(0.433)$ & $0.742(0.395)$ & $0.837(0.433)$ \\
\hline
\end{tabular}

Table 2.10: Simultaneous coverage probabilities (average median interval length) over $G_{\text {sub }}$ for $k=2$ and $a=0.3$. 


\begin{tabular}{|c|c|c|c|c|c|}
\hline \multirow[b]{2}{*}{$h$} & \multirow[b]{2}{*}{ DGP } & \multirow{2}{*}{$\frac{-}{\text { SWB }}$} & \multirow{2}{*}{$\begin{array}{c}\gamma=0 \\
\mathrm{WB}\end{array}$} & \multicolumn{2}{|c|}{$\gamma=0.2$} \\
\hline & & & & AWB & DWB \\
\hline \multirow{5}{*}{0.02} & 0 & $0.856(0.524)$ & $0.904(0.532)$ & $0.920(0.519)$ & $0.911(0.533)$ \\
\hline & $A R_{0.2}$ & $0.687(0.502)$ & $0.817(0.515)$ & $0.886(0.518)$ & $0.845(0.518)$ \\
\hline & $A R_{0.5}$ & $0.237(0.391)$ & $0.576(0.438)$ & $0.678(0.457)$ & $0.589(0.438)$ \\
\hline & $A R_{-0.5}$ & $0.975(0.402)$ & $0.978(0.454)$ & $0.981(0.417)$ & $0.979(0.453)$ \\
\hline & $M A_{0.2}$ & $0.702(0.502)$ & $0.836(0.517)$ & $0.880(0.518)$ & $0.849(0.518)$ \\
\hline \multirow{5}{*}{0.04} & 0 & $0.850(0.387)$ & $0.898(0.401)$ & $0.902(0.396)$ & $0.901(0.402)$ \\
\hline & $A R_{0.2}$ & $0.666(0.372)$ & $0.769(0.390)$ & $0.815(0.397)$ & $0.781(0.390)$ \\
\hline & $A R_{0.5}$ & $0.160(0.290)$ & $0.395(0.337)$ & $0.496(0.360)$ & $0.399(0.337)$ \\
\hline & $A R_{-0.5}$ & $0.988(0.298)$ & $0.993(0.350)$ & $0.988(0.323)$ & $0.986(0.347)$ \\
\hline & $M A_{0.2}$ & $0.701(0.371)$ & $0.797(0.391)$ & $0.824(0.397)$ & $0.789(0.391)$ \\
\hline \multirow{5}{*}{0.06} & 0 & $0.857(0.323)$ & $0.903(0.342)$ & $0.899(0.341)$ & $0.901(0.343)$ \\
\hline & $A R_{0.2}$ & $0.679(0.310)$ & $0.760(0.333)$ & $0.807(0.343)$ & $0.769(0.333)$ \\
\hline & $A R_{0.5}$ & $0.166(0.243)$ & $0.372(0.291)$ & $0.449(0.313)$ & $0.361(0.290)$ \\
\hline & $A R_{-0.5}$ & $0.995(0.249)$ & $0.997(0.301)$ & $0.995(0.283)$ & $0.997(0.300)$ \\
\hline & $M A_{0.2}$ & $0.715(0.310)$ & $0.798(0.333)$ & $0.811(0.343)$ & $0.795(0.334)$ \\
\hline & \multirow[b]{2}{*}{ DGP } & \multicolumn{2}{|c|}{$\gamma=0.4$} & \multicolumn{2}{|c|}{$\gamma=0.6$} \\
\hline & & AWB & DWB & AWB & DWB \\
\hline \multirow{5}{*}{0.02} & 0 & $0.905(0.477)$ & $0.914(0.532)$ & $0.810(0.403)$ & $0.925(0.533)$ \\
\hline & $A R_{0.2}$ & $0.869(0.486)$ & $0.830(0.515)$ & $0.748(0.424)$ & $0.829(0.517)$ \\
\hline & $A R_{0.5}$ & $0.638(0.449)$ & $0.555(0.435)$ & $0.574(0.411)$ & $0.566(0.437)$ \\
\hline & $A R_{-0.5}$ & $0.958(0.362)$ & $0.974(0.453)$ & $0.905(0.289)$ & $0.979(0.453)$ \\
\hline & $M A_{0.2}$ & $0.857(0.486)$ & $0.853(0.517)$ & $0.760(0.422)$ & $0.862(0.518)$ \\
\hline \multirow{5}{*}{0.04} & 0 & $0.864(0.371)$ & $0.908(0.401)$ & $0.729(0.323)$ & $0.901(0.402)$ \\
\hline & $A R_{0.2}$ & $0.775(0.382)$ & $0.765(0.389)$ & $0.665(0.342)$ & $0.755(0.390)$ \\
\hline & $A R_{0.5}$ & $0.495(0.365)$ & $0.389(0.335)$ & $0.465(0.345)$ & $0.403(0.335)$ \\
\hline & $A R_{-0.5}$ & $0.962(0.288)$ & $0.988(0.347)$ & $0.902(0.240)$ & $0.995(0.348)$ \\
\hline & $M A_{0.2}$ & $0.798(0.382)$ & $0.808(0.391)$ & $0.674(0.340)$ & $0.792(0.391)$ \\
\hline \multirow{5}{*}{0.06} & 0 & $0.857(0.325)$ & $0.903(0.342)$ & $0.729(0.290)$ & $0.897(0.342)$ \\
\hline & $A R_{0.2}$ & $0.752(0.335)$ & $0.747(0.332)$ & $0.630(0.307)$ & $0.757(0.333)$ \\
\hline & $A R_{0.5}$ & $0.496(0.322)$ & $0.375(0.289)$ & $0.434(0.312)$ & $0.391(0.289)$ \\
\hline & $A R_{-0.5}$ & $0.980(0.257)$ & $0.996(0.300)$ & $0.937(0.225)$ & $0.997(0.300)$ \\
\hline & $M A_{0.2}$ & $0.777(0.334)$ & $0.786(0.334)$ & $0.655(0.305)$ & $0.789(0.334)$ \\
\hline
\end{tabular}

Table 2.11: Simultaneous coverage probabilities (average median interval length) over $G$ for $k=2$ and $a=0.3$. 
2.C Additional simulation Results

\begin{tabular}{|c|c|c|c|c|c|}
\hline \multirow[b]{2}{*}{$h$} & \multirow[b]{2}{*}{ DGP } & \multirow{2}{*}{$\frac{-}{\text { SWB }}$} & \multirow{2}{*}{$\begin{array}{c}\gamma=0 \\
\mathrm{WB}\end{array}$} & \multicolumn{2}{|c|}{$\gamma=0.2$} \\
\hline & & & & AWB & DWB \\
\hline \multirow{5}{*}{0.02} & 0 & $0.920(0.702)$ & $0.946(0.721)$ & $0.952(0.706)$ & $0.946(0.723)$ \\
\hline & $A R_{0.2}$ & $0.857(0.674)$ & $0.901(0.700)$ & $0.918(0.702)$ & $0.902(0.702)$ \\
\hline & $A R_{0.5}$ & $0.671(0.526)$ & $0.780(0.594)$ & $0.819(0.619)$ & $0.778(0.594)$ \\
\hline & $A R_{-0.5}$ & $0.955(0.536)$ & $0.989(0.615)$ & $0.988(0.567)$ & $0.989(0.613)$ \\
\hline & $M A_{0.2}$ & $0.867(0.673)$ & $0.910(0.701)$ & $0.924(0.702)$ & $0.910(0.702)$ \\
\hline \multirow{5}{*}{0.04} & 0 & $0.916(0.526)$ & $0.950(0.557)$ & $0.951(0.553)$ & $0.951(0.558)$ \\
\hline & $A R_{0.2}$ & $0.857(0.507)$ & $0.900(0.542)$ & $0.911(0.553)$ & $0.900(0.542)$ \\
\hline & $A R_{0.5}$ & $0.663(0.398)$ & $0.767(0.470)$ & $0.806(0.501)$ & $0.770(0.469)$ \\
\hline & $A R_{-0.5}$ & $0.965(0.405)$ & $0.996(0.486)$ & $0.993(0.453)$ & $0.995(0.482)$ \\
\hline & $M A_{0.2}$ & $0.869(0.507)$ & $0.912(0.543)$ & $0.920(0.554)$ & $0.911(0.544)$ \\
\hline \multirow{5}{*}{0.06} & 0 & $0.921(0.440)$ & $0.955(0.478)$ & $0.955(0.480)$ & $0.955(0.479)$ \\
\hline & $A R_{0.2}$ & $0.858(0.424)$ & $0.906(0.466)$ & $0.918(0.481)$ & $0.908(0.466)$ \\
\hline & $A R_{0.5}$ & $0.663(0.333)$ & $0.778(0.409)$ & $0.816(0.441)$ & $0.782(0.409)$ \\
\hline & $A R_{-0.5}$ & $0.975(0.340)$ & $0.997(0.423)$ & $0.995(0.399)$ & $0.997(0.420)$ \\
\hline & $M A_{0.2}$ & $0.872(0.423)$ & $0.919(0.467)$ & $0.926(0.482)$ & $0.918(0.468)$ \\
\hline \multirow[b]{2}{*}{$h$} & & \multicolumn{2}{|c|}{$\gamma=0.4$} & \multicolumn{2}{|c|}{$\gamma=0.6$} \\
\hline & DGP & AWB & DWB & AWB & DWB \\
\hline \multirow{5}{*}{0.02} & 0 & $0.942(0.649)$ & $0.945(0.721)$ & $0.905(0.548)$ & $0.947(0.724)$ \\
\hline & $A R_{0.2}$ & $0.913(0.659)$ & $0.903(0.699)$ & $0.868(0.575)$ & $0.901(0.701)$ \\
\hline & $A R_{0.5}$ & $0.827(0.607)$ & $0.779(0.591)$ & $0.789(0.553)$ & $0.777(0.594)$ \\
\hline & $A R_{-0.5}$ & $0.983(0.497)$ & $0.989(0.614)$ & $0.960(0.399)$ & $0.990(0.613)$ \\
\hline & $M A_{0.2}$ & $0.918(0.660)$ & $0.908(0.702)$ & $0.879(0.571)$ & $0.912(0.703)$ \\
\hline \multirow{5}{*}{0.04} & 0 & $0.939(0.520)$ & $0.949(0.557)$ & $0.903(0.453)$ & $0.951(0.558)$ \\
\hline & $A R_{0.2}$ & $0.904(0.533)$ & $0.900(0.540)$ & $0.866(0.479)$ & $0.897(0.543)$ \\
\hline & $A R_{0.5}$ & $0.816(0.507)$ & $0.767(0.467)$ & $0.792(0.479)$ & $0.764(0.467)$ \\
\hline & $A R_{-0.5}$ & $0.988(0.408)$ & $0.995(0.482)$ & $0.968(0.342)$ & $0.995(0.483)$ \\
\hline & $M A_{0.2}$ & $0.911(0.533)$ & $0.909(0.543)$ & $0.877(0.476)$ & $0.911(0.544)$ \\
\hline \multirow{5}{*}{0.06} & 0 & $0.942(0.459$ & $0.952(0.47$ & $0.913(0.411)$ & $0.954(0.4$ \\
\hline & $A R_{0.2}$ & $0.911(0.470)$ & $0.905(0.465)$ & $0.879(0.433)$ & $0.903(0.466)$ \\
\hline & $A R_{0.5}$ & $0.827(0.452)$ & $0.775(0.406)$ & $0.814(0.439)$ & $0.775(0.407)$ \\
\hline & $A R_{-0.5}$ & $0.992(0.367)$ & $0.997(0.419)$ & $0.977(0.322)$ & $0.997(0.420)$ \\
\hline & $M A_{0.2}$ & $0.917(0.471)$ & $0.913(0.466)$ & $0.890(0.430)$ & $0.916(0.466)$ \\
\hline
\end{tabular}

Table 2.12: Pointwise coverage probabilities (average median interval length) for $k=2$ and $a=0.5$. 


\begin{tabular}{|c|c|c|c|c|c|}
\hline \multirow[b]{2}{*}{$h$} & \multirow[b]{2}{*}{ DGP } & \multirow{2}{*}{$\frac{-}{\mathrm{SWB}}$} & \multirow{2}{*}{$\begin{array}{c}\gamma=0 \\
\mathrm{WB}\end{array}$} & \multicolumn{2}{|c|}{$\gamma=0.2$} \\
\hline & & & & AWB & DWB \\
\hline \multirow{5}{*}{0.02} & 0 & $0.877(0.648)$ & $0.902(0.665)$ & $0.909(0.652)$ & $0.906(0.668)$ \\
\hline & $A R_{0.2}$ & $0.790(0.622)$ & $0.838(0.647)$ & $0.871(0.648)$ & $0.857(0.648)$ \\
\hline & $A R_{0.5}$ & $0.412(0.485)$ & $0.647(0.548)$ & $0.704(0.571)$ & $0.640(0.549)$ \\
\hline & $A R_{-0.5}$ & $0.979(0.495)$ & $0.985(0.568)$ & $0.983(0.524)$ & $0.980(0.566)$ \\
\hline & $M A_{0.2}$ & $0.782(0.622)$ & $0.844(0.648)$ & $0.872(0.648)$ & $0.842(0.649)$ \\
\hline \multirow{5}{*}{0.04} & 0 & $0.887(0.488)$ & $0.906(0.517)$ & $0.908(0.514)$ & $0.906(0.517)$ \\
\hline & $A R_{0.2}$ & $0.755(0.470)$ & $0.814(0.503)$ & $0.858(0.513)$ & $0.837(0.503)$ \\
\hline & $A R_{0.5}$ & $0.343(0.368)$ & $0.563(0.436)$ & $0.628(0.465)$ & $0.565(0.436)$ \\
\hline & $A R_{-0.5}$ & $0.988(0.375)$ & $0.994(0.450)$ & $0.989(0.420)$ & $0.988(0.448)$ \\
\hline & $M A_{0.2}$ & $0.775(0.470)$ & $0.827(0.504)$ & $0.853(0.515)$ & $0.826(0.505)$ \\
\hline \multirow{5}{*}{0.06} & 0 & $0.891(0.409)$ & $0.920(0.444)$ & $0.921(0.446)$ & $0.920(0.445)$ \\
\hline & $A R_{0.2}$ & $0.771(0.394)$ & $0.821(0.433)$ & $0.846(0.446)$ & $0.833(0.433)$ \\
\hline & $A R_{0.5}$ & $0.350(0.310)$ & $0.541(0.379)$ & $0.623(0.410)$ & $0.558(0.380)$ \\
\hline & $A R_{-0.5}$ & $0.998(0.315)$ & $0.999(0.392)$ & $0.994(0.371)$ & $0.995(0.390)$ \\
\hline & $M A_{0.2}$ & $0.792(0.394)$ & $0.833(0.434)$ & $0.856(0.448)$ & $0.854(0.435)$ \\
\hline & \multirow[b]{2}{*}{ DGP } & \multicolumn{2}{|c|}{$\gamma=0.4$} & \multicolumn{2}{|c|}{$\gamma=0.6$} \\
\hline & & AWB & DWB & AWB & DWB \\
\hline \multirow{5}{*}{0.02} & 0 & $0.892(0.598)$ & $0.916(0.667)$ & $0.790(0.506)$ & $0.913(0.668)$ \\
\hline & $A R_{0.2}$ & $0.835(0.608)$ & $0.838(0.645)$ & $0.764(0.529)$ & $0.854(0.647)$ \\
\hline & $A R_{0.5}$ & $0.684(0.560)$ & $0.631(0.546)$ & $0.620(0.509)$ & $0.643(0.549)$ \\
\hline & $A R_{-0.5}$ & $0.960(0.459)$ & $0.974(0.567)$ & $0.899(0.368)$ & $0.979(0.565)$ \\
\hline & $M A_{0.2}$ & $0.855(0.609)$ & $0.856(0.648)$ & $0.759(0.527)$ & $0.862(0.649)$ \\
\hline \multirow{5}{*}{0.04} & 0 & $0.888(0.482)$ & $0.926(0.517)$ & $0.778(0.420)$ & $0.915(0.517)$ \\
\hline & $A R_{0.2}$ & $0.805(0.494)$ & $0.819(0.502)$ & $0.745(0.444)$ & $0.835(0.503)$ \\
\hline & $A R_{0.5}$ & $0.644(0.470)$ & $0.552(0.433)$ & $0.578(0.443)$ & $0.548(0.434)$ \\
\hline & $A R_{-0.5}$ & $0.971(0.379)$ & $0.989(0.447)$ & $0.931(0.317)$ & $0.990(0.448)$ \\
\hline & $M A_{0.2}$ & $0.832(0.495)$ & $0.861(0.504)$ & $0.722(0.441)$ & $0.832(0.505)$ \\
\hline \multirow{5}{*}{0.06} & 0 & $0.889(0.426)$ & $0.926(0.444)$ & $0.798(0.381)$ & $0.916(0.444)$ \\
\hline & $A R_{0.2}$ & $0.810(0.436)$ & $0.827(0.432)$ & $0.753(0.402)$ & $0.836(0.433)$ \\
\hline & $A R_{0.5}$ & $0.657(0.420)$ & $0.556(0.377)$ & $0.608(0.407)$ & $0.556(0.378)$ \\
\hline & $A R_{-0.5}$ & $0.986(0.341)$ & $0.997(0.390)$ & $0.958(0.298)$ & $0.999(0.390)$ \\
\hline & $M A_{0.2}$ & $0.848(0.438)$ & $0.859(0.433)$ & $0.752(0.399)$ & $0.845(0.434)$ \\
\hline
\end{tabular}

Table 2.13: Simultaneous coverage probabilities (average median interval length) over $G_{\text {sub }}$ for $k=2$ and $a=0.5$. 
2.C Additional simulation Results

\begin{tabular}{|c|c|c|c|c|c|}
\hline \multirow[b]{2}{*}{$h$} & \multirow[b]{2}{*}{ DGP } & \multirow{2}{*}{$\frac{-}{\text { SWB }}$} & \multirow{2}{*}{$\begin{array}{c}\gamma=0 \\
\mathrm{WB}\end{array}$} & \multicolumn{2}{|c|}{$\gamma=0.2$} \\
\hline & & & & AWB & DWB \\
\hline \multirow{5}{*}{0.02} & 0 & $0.845(0.513)$ & $0.898(0.527)$ & $0.912(0.516)$ & $0.908(0.528)$ \\
\hline & $A R_{0.2}$ & $0.682(0.492)$ & $0.810(0.511)$ & $0.875(0.513)$ & $0.841(0.513)$ \\
\hline & $A R_{0.5}$ & $0.241(0.384)$ & $0.574(0.433)$ & $0.665(0.453)$ & $0.581(0.434)$ \\
\hline & $A R_{-0.5}$ & $0.974(0.392)$ & $0.977(0.449)$ & $0.981(0.414)$ & $0.979(0.448)$ \\
\hline & $M A_{0.2}$ & $0.709(0.492)$ & $0.829(0.512)$ & $0.872(0.513)$ & $0.842(0.514)$ \\
\hline \multirow{5}{*}{0.04} & 0 & $0.849(0.378)$ & $0.895(0.401)$ & $0.897(0.398)$ & $0.905(0.402)$ \\
\hline & $A R_{0.2}$ & $0.662(0.365)$ & $0.767(0.390)$ & $0.812(0.398)$ & $0.784(0.390)$ \\
\hline & $A R_{0.5}$ & $0.167(0.286)$ & $0.396(0.338)$ & $0.493(0.360)$ & $0.403(0.337)$ \\
\hline & $A R_{-0.5}$ & $0.989(0.291)$ & $0.993(0.349)$ & $0.988(0.326)$ & $0.988(0.347)$ \\
\hline & $M A_{0.2}$ & $0.702(0.364)$ & $0.787(0.391)$ & $0.822(0.398)$ & $0.789(0.391)$ \\
\hline \multirow{5}{*}{0.06} & 0 & $0.858(0.314)$ & $0.899(0.342)$ & $0.899(0.343)$ & $0.902(0.343)$ \\
\hline & $A R_{0.2}$ & $0.685(0.303)$ & $0.761(0.333)$ & $0.809(0.344)$ & $0.771(0.333)$ \\
\hline & $A R_{0.5}$ & $0.159(0.238)$ & $0.374(0.292)$ & $0.452(0.316)$ & $0.361(0.293)$ \\
\hline & $A R_{-0.5}$ & $0.994(0.242)$ & $0.997(0.301)$ & $0.996(0.285)$ & $0.997(0.300)$ \\
\hline & $M A_{0.2}$ & $0.718(0.302)$ & $0.794(0.334)$ & $0.815(0.344)$ & $0.796(0.335)$ \\
\hline \multirow[b]{2}{*}{$h$} & & \multicolumn{2}{|c|}{$\gamma=0.4$} & \multicolumn{2}{|c|}{$\gamma=0.6$} \\
\hline & DGP & AWB & DWB & AWB & DWB \\
\hline \multirow{5}{*}{0.02} & 0 & $0.897(0.474)$ & $0.908(0.528)$ & $0.789(0.400)$ & $0.914(0.529)$ \\
\hline & $A R_{0.2}$ & $0.857(0.482)$ & $0.824(0.511)$ & $0.736(0.420)$ & $0.824(0.512)$ \\
\hline & $A R_{0.5}$ & $0.624(0.444)$ & $0.545(0.432)$ & $0.560(0.405)$ & $0.554(0.434)$ \\
\hline & $A R_{-0.5}$ & $0.959(0.362)$ & $0.975(0.448)$ & $0.901(0.291)$ & $0.979(0.448)$ \\
\hline & $M A_{0.2}$ & $0.846(0.483)$ & $0.847(0.512)$ & $0.738(0.418)$ & $0.864(0.514)$ \\
\hline \multirow{5}{*}{0.04} & 0 & $0.863(0.374)$ & $0.902(0.400)$ & $0.732(0.326)$ & $0.902(0.402)$ \\
\hline & $A R_{0.2}$ & $0.768(0.383)$ & $0.761(0.389)$ & $0.657(0.345)$ & $0.757(0.390)$ \\
\hline & $A R_{0.5}$ & $0.509(0.365)$ & $0.398(0.336)$ & $0.458(0.345)$ & $0.408(0.336)$ \\
\hline & $A R_{-0.5}$ & $0.965(0.294)$ & $0.989(0.347)$ & $0.903(0.246)$ & $0.995(0.347)$ \\
\hline & $M A_{0.2}$ & $0.796(0.384)$ & $0.807(0.391)$ & $0.664(0.343)$ & $0.791(0.391)$ \\
\hline \multirow{5}{*}{0.06} & 0 & $0.854(0.328)$ & $0.900(0.341)$ & $0.730(0.294)$ & $0.901(0.342)$ \\
\hline & $A R_{0.2}$ & $0.740(0.337)$ & $0.748(0.333)$ & $0.628(0.310)$ & $0.764(0.333)$ \\
\hline & $A R_{0.5}$ & $0.503(0.324)$ & $0.383(0.290)$ & $0.442(0.314)$ & $0.397(0.291)$ \\
\hline & $A R_{-0.5}$ & $0.980(0.262)$ & $0.996(0.300)$ & $0.934(0.230)$ & $0.998(0.300)$ \\
\hline & $M A_{0.2}$ & $0.772(0.337)$ & $0.791(0.333)$ & $0.662(0.308)$ & $0.787(0.334)$ \\
\hline
\end{tabular}

Table 2.14: Simultaneous coverage probabilities (average median interval length) over $G$ for $k=2$ and $a=0.5$. 


\begin{tabular}{|c|c|c|c|c|c|}
\hline \multirow[b]{2}{*}{$h$} & \multirow[b]{2}{*}{ DGP } & \multirow{2}{*}{$\frac{-}{\mathrm{SWB}}$} & \multirow{2}{*}{$\begin{array}{c}\gamma=0 \\
\mathrm{WB}\end{array}$} & \multicolumn{2}{|c|}{$\gamma=0.2$} \\
\hline & & & & AWB & DWB \\
\hline \multirow{5}{*}{0.02} & 0 & $0.914(0.685)$ & $0.945(0.713)$ & $0.951(0.699)$ & $0.945(0.715)$ \\
\hline & $A R_{0.2}$ & $0.850(0.657)$ & $0.901(0.692)$ & $0.918(0.696)$ & $0.902(0.693)$ \\
\hline & $A R_{0.5}$ & $0.666(0.512)$ & $0.781(0.588)$ & $0.819(0.614)$ & $0.779(0.590)$ \\
\hline & $A R_{-0.5}$ & $0.950(0.520)$ & $0.990(0.607)$ & $0.988(0.562)$ & $0.989(0.605)$ \\
\hline & $M A_{0.2}$ & $0.861(0.656)$ & $0.910(0.694)$ & $0.924(0.696)$ & $0.910(0.695)$ \\
\hline \multirow{5}{*}{0.04} & 0 & $0.910(0.510)$ & $0.950(0.553)$ & $0.951(0.552)$ & $0.951(0.554)$ \\
\hline & $A R_{0.2}$ & $0.852(0.494)$ & $0.901(0.541)$ & $0.911(0.552)$ & $0.901(0.540)$ \\
\hline & $A R_{0.5}$ & $0.658(0.387)$ & $0.769(0.469)$ & $0.808(0.502)$ & $0.772(0.470)$ \\
\hline & $A R_{-0.5}$ & $0.961(0.393)$ & $0.996(0.482)$ & $0.993(0.452)$ & $0.995(0.479)$ \\
\hline & $M A_{0.2}$ & $0.862(0.493)$ & $0.913(0.541)$ & $0.920(0.554)$ & $0.912(0.542)$ \\
\hline \multirow{5}{*}{0.06} & 0 & $0.915(0.426)$ & $0.956(0.476)$ & $0.955(0.480)$ & $0.956(0.477)$ \\
\hline & $A R_{0.2}$ & $0.853(0.412)$ & $0.907(0.465)$ & $0.918(0.481)$ & $0.908(0.465)$ \\
\hline & $A R_{0.5}$ & $0.658(0.323)$ & $0.780(0.410)$ & $0.819(0.443)$ & $0.784(0.411)$ \\
\hline & $A R_{-0.5}$ & $0.971(0.329)$ & $0.997(0.421)$ & $0.996(0.400)$ & $0.997(0.418)$ \\
\hline & $M A_{0.2}$ & $0.866(0.409)$ & $0.919(0.465)$ & $0.927(0.482)$ & $0.918(0.467)$ \\
\hline \multirow[b]{2}{*}{$h$} & \multirow[b]{2}{*}{ DGP } & \multicolumn{2}{|c|}{$\gamma=0.4$} & \multicolumn{2}{|c|}{$\gamma=0.6$} \\
\hline & & AWB & DWB & AWB & DWB \\
\hline \multirow{5}{*}{0.02} & 0 & $0.941(0.645)$ & $0.944(0.7$ & $0.903(0.546)$ & $0.947(0.715)$ \\
\hline & $A R_{0.2}$ & $0.911(0.654)$ & $0.903(0.692)$ & $0.866(0.571)$ & $0.900(0.693)$ \\
\hline & $A R_{0.5}$ & $0.827(0.601)$ & $0.780(0.586)$ & $0.789(0.548)$ & $0.778(0.589)$ \\
\hline & $A R_{-0.5}$ & $0.983(0.496)$ & $0.989(0.604)$ & $0.960(0.403)$ & $0.990(0.604)$ \\
\hline & $M A_{0.2}$ & $0.916(0.656)$ & $0.908(0.695)$ & $0.877(0.568)$ & $0.912(0.695)$ \\
\hline \multirow{5}{*}{0.04} & 0 & $0.939(0.523)$ & $0.949(0.553)$ & $0.905(0.458)$ & $0.951(0.554)$ \\
\hline & $A R_{0.2}$ & $0.905(0.534)$ & $0.901(0.538)$ & $0.867(0.483)$ & $0.898(0.540)$ \\
\hline & $A R_{0.5}$ & $0.818(0.508)$ & $0.768(0.467)$ & $0.795(0.481)$ & $0.767(0.468)$ \\
\hline & $A R_{-0.5}$ & $0.989(0.414)$ & $0.995(0.479)$ & $0.968(0.351)$ & $0.996(0.479)$ \\
\hline & $M A_{0.2}$ & $0.911(0.536)$ & $0.909(0.541)$ & $0.878(0.480)$ & $0.912(0.541)$ \\
\hline \multirow{5}{*}{0.06} & 0 & $0.943(0.462)$ & $0.952(0.475)$ & $0.915(0.416)$ & $0.954(0.476)$ \\
\hline & $A R_{0.2}$ & $0.912(0.472)$ & $0.906(0.464)$ & $0.881(0.438)$ & $0.904(0.466)$ \\
\hline & $A R_{0.5}$ & $0.829(0.455)$ & $0.778(0.407)$ & $0.817(0.442)$ & $0.778(0.408)$ \\
\hline & $A R_{-0.5}$ & $0.992(0.372)$ & $0.997(0.418)$ & $0.978(0.329)$ & $0.998(0.418)$ \\
\hline & $M A_{0.2}$ & $0.918(0.474)$ & $0.914(0.465)$ & $0.891(0.435)$ & $0.916(0.465)$ \\
\hline
\end{tabular}

Table 2.15: Pointwise coverage probabilities (average median interval length) for $k=2$ and $a=0.7$. 


\begin{tabular}{|c|c|c|c|c|c|}
\hline \multirow[b]{2}{*}{$h$} & \multirow[b]{2}{*}{ DGP } & \multirow{2}{*}{$\frac{-}{\text { SWB }}$} & \multirow{2}{*}{$\begin{array}{c}\gamma=0 \\
\mathrm{WB}\end{array}$} & \multicolumn{2}{|c|}{$\gamma=0.2$} \\
\hline & & & & AWB & DWB \\
\hline \multirow{5}{*}{0.02} & 0 & $0.877(0.633)$ & $0.894(0.658)$ & $0.904(0.645)$ & $0.893(0.660)$ \\
\hline & $A R_{0.2}$ & $0.781(0.607)$ & $0.822(0.640)$ & $0.856(0.641)$ & $0.851(0.641)$ \\
\hline & $A R_{0.5}$ & $0.415(0.473)$ & $0.637(0.542)$ & $0.696(0.567)$ & $0.641(0.545)$ \\
\hline & $A R_{-0.5}$ & $0.981(0.480)$ & $0.983(0.561)$ & $0.982(0.520)$ & $0.981(0.559)$ \\
\hline & $M A_{0.2}$ & $0.790(0.606)$ & $0.834(0.640)$ & $0.859(0.642)$ & $0.835(0.641)$ \\
\hline \multirow{5}{*}{0.04} & 0 & $0.897(0.474)$ & $0.909(0.513)$ & $0.909(0.513)$ & $0.909(0.514)$ \\
\hline & $A R_{0.2}$ & $0.769(0.459)$ & $0.822(0.501)$ & $0.851(0.512)$ & $0.840(0.501)$ \\
\hline & $A R_{0.5}$ & $0.365(0.359)$ & $0.571(0.436)$ & $0.639(0.465)$ & $0.575(0.437)$ \\
\hline & $A R_{-0.5}$ & $0.990(0.364)$ & $0.993(0.448)$ & $0.988(0.420)$ & $0.989(0.444)$ \\
\hline & $M A_{0.2}$ & $0.783(0.457)$ & $0.829(0.502)$ & $0.850(0.514)$ & $0.830(0.502)$ \\
\hline \multirow{5}{*}{0.06} & 0 & $0.895(0.396)$ & $0.923(0.442)$ & $0.923(0.447)$ & $0.921(0.443)$ \\
\hline & $A R_{0.2}$ & $0.783(0.383)$ & $0.824(0.433)$ & $0.858(0.447)$ & $0.842(0.433)$ \\
\hline & $A R_{0.5}$ & $0.364(0.301)$ & $0.556(0.381)$ & $0.632(0.411)$ & $0.571(0.381)$ \\
\hline & $A R_{-0.5}$ & $0.997(0.306)$ & $0.999(0.391)$ & $0.997(0.372)$ & $0.998(0.389)$ \\
\hline & $M A_{0.2}$ & $0.799(0.381)$ & $0.830(0.433)$ & $0.870(0.449)$ & $0.852(0.434)$ \\
\hline & \multirow[b]{2}{*}{ DGP } & \multicolumn{2}{|c|}{$\gamma=0.4$} & \multicolumn{2}{|c|}{$\gamma=0.6$} \\
\hline & & AWB & DWB & AWB & DWB \\
\hline \multirow{5}{*}{0.02} & 0 & $0.872(0.596)$ & $0.907(0.660)$ & $0.781(0.504)$ & $0.907(0.660)$ \\
\hline & $A R_{0.2}$ & $0.819(0.604)$ & $0.832(0.639)$ & $0.744(0.526)$ & $0.852(0.640)$ \\
\hline & $A R_{0.5}$ & $0.673(0.555)$ & $0.632(0.542)$ & $0.598(0.504)$ & $0.625(0.544)$ \\
\hline & $A R_{-0.5}$ & $0.960(0.458)$ & $0.975(0.558)$ & $0.909(0.371)$ & $0.983(0.558)$ \\
\hline & $M A_{0.2}$ & $0.838(0.606)$ & $0.849(0.642)$ & $0.743(0.523)$ & $0.853(0.642)$ \\
\hline \multirow{5}{*}{0.04} & 0 & $0.884(0.485)$ & $0.923(0.513)$ & $0.780(0.424)$ & $0.910(0.513)$ \\
\hline & $A R_{0.2}$ & $0.807(0.496)$ & $0.819(0.499)$ & $0.751(0.448)$ & $0.840(0.502)$ \\
\hline & $A R_{0.5}$ & $0.646(0.471)$ & $0.562(0.434)$ & $0.580(0.445)$ & $0.562(0.435)$ \\
\hline & $A R_{-0.5}$ & $0.975(0.384)$ & $0.990(0.445)$ & $0.943(0.325)$ & $0.992(0.445)$ \\
\hline & $M A_{0.2}$ & $0.838(0.497)$ & $0.857(0.502)$ & $0.728(0.444)$ & $0.833(0.502)$ \\
\hline \multirow{5}{*}{0.06} & 0 & $0.901(0.429)$ & $0.927(0.442)$ & $0.811(0.386)$ & $0.914(0.442)$ \\
\hline & $A R_{0.2}$ & $0.821(0.439)$ & $0.833(0.431)$ & $0.764(0.407)$ & $0.842(0.433)$ \\
\hline & $A R_{0.5}$ & $0.665(0.423)$ & $0.568(0.378)$ & $0.619(0.410)$ & $0.572(0.380)$ \\
\hline & $A R_{-0.5}$ & $0.988(0.346)$ & $0.997(0.389)$ & $0.965(0.305)$ & $1.000(0.388)$ \\
\hline & $M A_{0.2}$ & $0.851(0.441)$ & $0.868(0.433)$ & $0.770(0.403)$ & $0.847(0.433)$ \\
\hline
\end{tabular}

Table 2.16: Simultaneous coverage probabilities (average median interval length) over $G_{\text {sub }}$ for $k=2$ and $a=0.7$. 


\begin{tabular}{|c|c|c|c|c|c|}
\hline \multirow[b]{2}{*}{$h$} & \multirow[b]{2}{*}{ DGP } & \multirow{2}{*}{$\frac{-}{\mathrm{SWB}}$} & \multirow{2}{*}{$\begin{array}{c}\gamma=0 \\
\mathrm{WB}\end{array}$} & \multicolumn{2}{|c|}{$\gamma=0.2$} \\
\hline & & & & AWB & DWB \\
\hline \multirow{5}{*}{0.02} & 0 & $0.847(0.501)$ & $0.887(0.521)$ & $0.906(0.511)$ & $0.898(0.522)$ \\
\hline & $A R_{0.2}$ & $0.682(0.480)$ & $0.797(0.506)$ & $0.868(0.508)$ & $0.829(0.507)$ \\
\hline & $A R_{0.5}$ & $0.243(0.374)$ & $0.561(0.430)$ & $0.654(0.449)$ & $0.569(0.431)$ \\
\hline & $A R_{-0.5}$ & $0.977(0.380)$ & $0.977(0.444)$ & $0.983(0.411)$ & $0.977(0.442)$ \\
\hline & $M A_{0.2}$ & $0.713(0.480)$ & $0.823(0.507)$ & $0.866(0.509)$ & $0.837(0.508)$ \\
\hline \multirow{5}{*}{0.04} & 0 & $0.856(0.367)$ & $0.897(0.398)$ & $0.895(0.397)$ & $0.907(0.399)$ \\
\hline & $A R_{0.2}$ & $0.664(0.356)$ & $0.762(0.388)$ & $0.806(0.398)$ & $0.791(0.388)$ \\
\hline & $A R_{0.5}$ & $0.161(0.278)$ & $0.407(0.337)$ & $0.501(0.361)$ & $0.411(0.338)$ \\
\hline & $A R_{-0.5}$ & $0.990(0.282)$ & $0.993(0.346)$ & $0.988(0.325)$ & $0.989(0.344)$ \\
\hline & $M A_{0.2}$ & $0.694(0.354)$ & $0.785(0.389)$ & $0.827(0.398)$ & $0.795(0.389)$ \\
\hline \multirow{5}{*}{0.06} & 0 & $0.871(0.303)$ & $0.905(0.340)$ & $0.897(0.343)$ & $0.908(0.341)$ \\
\hline & $A R_{0.2}$ & $0.692(0.294)$ & $0.766(0.332)$ & $0.817(0.344)$ & $0.767(0.333)$ \\
\hline & $A R_{0.5}$ & $0.161(0.231)$ & $0.384(0.293)$ & $0.462(0.317)$ & $0.372(0.293)$ \\
\hline & $A R_{-0.5}$ & $0.994(0.235)$ & $0.998(0.300)$ & $0.997(0.286)$ & $0.998(0.299)$ \\
\hline & $M A_{0.2}$ & $0.716(0.292)$ & $0.792(0.333)$ & $0.815(0.345)$ & $0.795(0.334)$ \\
\hline & \multirow[b]{2}{*}{ DGP } & \multicolumn{2}{|c|}{$\gamma=0.4$} & \multicolumn{2}{|c|}{$\gamma=0.6$} \\
\hline & & AWB & DWB & AWB & DWB \\
\hline \multirow{5}{*}{0.02} & 0 & $0.880(0.472)$ & $0.905(0.521)$ & $0.781(0.399)$ & $0.904(0.523)$ \\
\hline & $A R_{0.2}$ & $0.840(0.479)$ & $0.810(0.506)$ & $0.724(0.418)$ & $0.812(0.506)$ \\
\hline & $A R_{0.5}$ & $0.612(0.440)$ & $0.534(0.429)$ & $0.546(0.401)$ & $0.547(0.430)$ \\
\hline & $A R_{-0.5}$ & $0.961(0.362)$ & $0.977(0.442)$ & $0.913(0.294)$ & $0.983(0.442)$ \\
\hline & $M A_{0.2}$ & $0.825(0.480)$ & $0.836(0.507)$ & $0.726(0.415)$ & $0.853(0.508)$ \\
\hline \multirow{5}{*}{0.04} & 0 & $0.860(0.376)$ & $0.901(0.397)$ & $0.736(0.330)$ & $0.908(0.398)$ \\
\hline & $A R_{0.2}$ & $0.770(0.385)$ & $0.768(0.387)$ & $0.656(0.348)$ & $0.758(0.388)$ \\
\hline & $A R_{0.5}$ & $0.522(0.365)$ & $0.401(0.336)$ & $0.463(0.347)$ & $0.415(0.337)$ \\
\hline & $A R_{-0.5}$ & $0.967(0.297)$ & $0.989(0.345)$ & $0.919(0.253)$ & $0.994(0.344)$ \\
\hline & $M A_{0.2}$ & $0.794(0.386)$ & $0.807(0.388)$ & $0.671(0.345)$ & $0.796(0.389)$ \\
\hline \multirow{5}{*}{0.06} & 0 & $0.857(0.330)$ & $0.896(0.339)$ & $0.739(0.298)$ & $0.902(0.340)$ \\
\hline & $A R_{0.2}$ & $0.753(0.338)$ & $0.751(0.331)$ & $0.637(0.313)$ & $0.767(0.333)$ \\
\hline & $A R_{0.5}$ & $0.514(0.325)$ & $0.392(0.291)$ & $0.457(0.316)$ & $0.407(0.292)$ \\
\hline & $A R_{-0.5}$ & $0.979(0.266)$ & $0.996(0.299)$ & $0.938(0.235)$ & $0.998(0.298)$ \\
\hline & $M A_{0.2}$ & $0.770(0.339)$ & $0.797(0.332)$ & $0.668(0.311)$ & $0.797(0.332)$ \\
\hline
\end{tabular}

Table 2.17: Simultaneous coverage probabilities (average median interval length) over $G$ for $k=2$ and $a=0.7$. 
2.C Additional simulation Results

\begin{tabular}{|c|c|c|c|c|c|}
\hline \multirow[b]{2}{*}{$h$} & \multirow[b]{2}{*}{ DGP } & \multirow{2}{*}{$\frac{-}{\text { SWB }}$} & \multirow{2}{*}{$\begin{array}{c}\gamma=0 \\
\mathrm{WB}\end{array}$} & \multicolumn{2}{|c|}{$\gamma=0.2$} \\
\hline & & & & AWB & DWB \\
\hline \multirow{5}{*}{0.02} & 0 & $0.927(0.719)$ & $0.946(0.728)$ & $0.952(0.712)$ & $0.946(0.730)$ \\
\hline & $A R_{0.2}$ & $0.862(0.690)$ & $0.902(0.708)$ & $0.919(0.710)$ & $0.902(0.709)$ \\
\hline & $A R_{0.5}$ & $0.674(0.538)$ & $0.780(0.601)$ & $0.819(0.626)$ & $0.778(0.601)$ \\
\hline & $A R_{-0.5}$ & $0.960(0.554)$ & $0.990(0.623)$ & $0.988(0.571)$ & $0.989(0.620)$ \\
\hline & $M A_{0.2}$ & $0.873(0.689)$ & $0.911(0.709)$ & $0.925(0.709)$ & $0.911(0.710)$ \\
\hline \multirow{5}{*}{0.04} & 0 & $0.923(0.541)$ & $0.950(0.553)$ & $0.950(0.545)$ & $0.951(0.554)$ \\
\hline & $A R_{0.2}$ & $0.862(0.519)$ & $0.900(0.540)$ & $0.911(0.548)$ & $0.900(0.540)$ \\
\hline & $A R_{0.5}$ & $0.667(0.405)$ & $0.765(0.466)$ & $0.804(0.498)$ & $0.768(0.466)$ \\
\hline & $A R_{-0.5}$ & $0.971(0.418)$ & $0.996(0.481)$ & $0.993(0.440)$ & $0.995(0.479)$ \\
\hline & $M A_{0.2}$ & $0.874(0.518)$ & $0.912(0.540)$ & $0.919(0.547)$ & $0.911(0.541)$ \\
\hline \multirow{5}{*}{0.06} & 0 & $926(0.455)$ & $0.956(0.471)$ & $0.955(0.468)$ & $0.955(0.472)$ \\
\hline & $A R_{0.2}$ & $0.866(0.437)$ & $0.907(0.461)$ & $0.918(0.473)$ & $0.908(0.461)$ \\
\hline & $A R_{0.5}$ & $0.668(0.341)$ & $0.779(0.403)$ & $0.815(0.435)$ & $0.781(0.403)$ \\
\hline & $A R_{-0.5}$ & $0.981(0.352)$ & $0.997(0.414)$ & $0.995(0.383)$ & $0.997(0.413)$ \\
\hline & $M A_{0.2}$ & $0.878(0.436)$ & $0.919(0.460)$ & $0.926(0.472)$ & $0.918(0.461)$ \\
\hline \multirow[b]{2}{*}{$h$} & & \multicolumn{2}{|c|}{$\gamma=0.4$} & \multicolumn{2}{|c|}{$\gamma=0.6$} \\
\hline & DGP & AWB & DWB & AWB & DWB \\
\hline \multirow{5}{*}{0.02} & 0 & $0.944(0.653)$ & $0.945(0.730)$ & $0.906(0.550)$ & $0.947(0.731)$ \\
\hline & $A R_{0.2}$ & $0.914(0.665)$ & $0.903(0.705)$ & $0.869(0.578)$ & $0.901(0.707)$ \\
\hline & $A R_{0.5}$ & $0.829(0.614)$ & $0.780(0.597)$ & $0.791(0.561)$ & $0.778(0.599)$ \\
\hline & $A R_{-0.5}$ & $0.983(0.493)$ & $0.989(0.619)$ & $0.959(0.392)$ & $0.989(0.622)$ \\
\hline & $M A_{0.2}$ & $0.919(0.665)$ & $0.909(0.709)$ & $0.880(0.576)$ & $0.912(0.710)$ \\
\hline \multirow{5}{*}{0.04} & 0 & $0.938(0.509)$ & $0.948(0.553)$ & $0.902(0.442)$ & $0.950(0.553)$ \\
\hline & $A R_{0.2}$ & $0.904(0.525)$ & $0.900(0.536)$ & $0.865(0.470)$ & $0.897(0.537)$ \\
\hline & $A R_{0.5}$ & $0.816(0.504)$ & $0.766(0.463)$ & $0.792(0.474)$ & $0.764(0.463)$ \\
\hline & $A R_{-0.5}$ & $0.988(0.385)$ & $0.995(0.479)$ & $0.967(0.318)$ & $0.995(0.481)$ \\
\hline & $M A_{0.2}$ & $0.911(0.526)$ & $0.909(0.540)$ & $0.877(0.468)$ & $0.911(0.539)$ \\
\hline \multirow{5}{*}{0.06} & 0 & $0.942(0.444)$ & $0.952(0.47$ & $0.913(0.397)$ & $0.954(0$. \\
\hline & $A R_{0.2}$ & $0.910(0.461)$ & $0.905(0.459)$ & $0.879(0.423)$ & $0.904(0.460)$ \\
\hline & $A R_{0.5}$ & $0.826(0.447)$ & $0.777(0.401)$ & $0.813(0.431)$ & $0.776(0.401)$ \\
\hline & $A R_{-0.5}$ & $0.991(0.343)$ & $0.997(0.413)$ & $0.979(0.299)$ & $0.997(0.413)$ \\
\hline & $M A_{0.2}$ & $0.917(0.460)$ & $0.913(0.461)$ & $0.889(0.419)$ & $0.916(0.460)$ \\
\hline
\end{tabular}

Table 2.18: Pointwise coverage probabilities (average median interval length) for $k=3$ and $a=0.3$. 


\begin{tabular}{|c|c|c|c|c|c|}
\hline \multirow[b]{2}{*}{$h$} & \multirow[b]{2}{*}{ DGP } & \multirow{2}{*}{$\frac{-}{\mathrm{SWB}}$} & \multirow{2}{*}{$\begin{array}{c}\gamma=0 \\
\mathrm{WB}\end{array}$} & \multicolumn{2}{|c|}{$\gamma=0.2$} \\
\hline & & & & AWB & DWB \\
\hline \multirow{5}{*}{0.02} & 0 & $0.879(0.664)$ & $0.905(0.672)$ & $0.915(0.657)$ & $0.912(0.674)$ \\
\hline & $A R_{0.2}$ & $0.787(0.636)$ & $0.845(0.653)$ & $0.880(0.655)$ & $0.843(0.655)$ \\
\hline & $A R_{0.5}$ & $0.406(0.497)$ & $0.657(0.555)$ & $0.713(0.577)$ & $0.643(0.554)$ \\
\hline & $A R_{-0.5}$ & $0.973(0.511)$ & $0.985(0.575)$ & $0.981(0.527)$ & $0.981(0.573)$ \\
\hline & $M A_{0.2}$ & $0.783(0.636)$ & $0.849(0.654)$ & $0.878(0.654)$ & $0.852(0.655)$ \\
\hline \multirow{5}{*}{0.04} & 0 & $0.889(0.502)$ & $0.911(0.513)$ & $0.909(0.506)$ & $0.912(0.514)$ \\
\hline & $A R_{0.2}$ & $0.753(0.482)$ & $0.817(0.501)$ & $0.858(0.508)$ & $0.837(0.500)$ \\
\hline & $A R_{0.5}$ & $0.334(0.376)$ & $0.561(0.433)$ & $0.626(0.462)$ & $0.559(0.432)$ \\
\hline & $A R_{-0.5}$ & $0.990(0.388)$ & $0.994(0.446)$ & $0.984(0.408)$ & $0.986(0.445)$ \\
\hline & $M A_{0.2}$ & $0.767(0.482)$ & $0.827(0.501)$ & $0.840(0.509)$ & $0.824(0.502)$ \\
\hline \multirow{5}{*}{0.06} & 0 & $0.878(0.424)$ & $0.917(0.438)$ & $0.921(0.435)$ & $0.923(0.439)$ \\
\hline & $A R_{0.2}$ & $0.770(0.406)$ & $0.813(0.428)$ & $0.833(0.439)$ & $0.835(0.429)$ \\
\hline & $A R_{0.5}$ & $0.339(0.317)$ & $0.536(0.374)$ & $0.603(0.404)$ & $0.547(0.374)$ \\
\hline & $A R_{-0.5}$ & $0.998(0.328)$ & $0.998(0.385)$ & $0.990(0.356)$ & $0.993(0.383)$ \\
\hline & $M A_{0.2}$ & $0.791(0.406)$ & $0.832(0.428)$ & $0.860(0.438)$ & $0.852(0.429)$ \\
\hline & \multirow[b]{2}{*}{ DGP } & \multicolumn{2}{|c|}{$\gamma=0.4$} & \multicolumn{2}{|c|}{$\gamma=0.6$} \\
\hline & & AWB & DWB & AWB & DWB \\
\hline \multirow{5}{*}{0.02} & 0 & $0.904(0.603)$ & $0.917(0.6$ & $0.814(0.508)$ & $0.922(0.675)$ \\
\hline & $A R_{0.2}$ & $0.842(0.614)$ & $0.853(0.651)$ & $0.778(0.532)$ & $0.861(0.653)$ \\
\hline & $A R_{0.5}$ & $0.704(0.567)$ & $0.643(0.552)$ & $0.609(0.516)$ & $0.640(0.553)$ \\
\hline & $A R_{-0.5}$ & $0.960(0.455)$ & $0.976(0.572)$ & $0.904(0.361)$ & $0.980(0.575)$ \\
\hline & $M A_{0.2}$ & $0.871(0.614)$ & $0.860(0.655)$ & $0.776(0.531)$ & $0.868(0.655)$ \\
\hline \multirow{5}{*}{0.04} & 0 & $0.883(0.471)$ & $0.928(0.514)$ & $0.762(0.409)$ & $0.916(0.513)$ \\
\hline & $A R_{0.2}$ & $0.806(0.488)$ & $0.816(0.499)$ & $0.751(0.436)$ & $0.834(0.499)$ \\
\hline & $A R_{0.5}$ & $0.630(0.467)$ & $0.538(0.430)$ & $0.574(0.439)$ & $0.557(0.430)$ \\
\hline & $A R_{-0.5}$ & $0.962(0.357)$ & $0.987(0.444)$ & $0.921(0.295)$ & $0.987(0.446)$ \\
\hline & $M A_{0.2}$ & $0.838(0.487)$ & $0.856(0.500)$ & $0.716(0.433)$ & $0.831(0.500)$ \\
\hline \multirow{5}{*}{0.06} & 0 & $0.900(0.413)$ & $0.930(0.439)$ & $0.787(0.368)$ & $0.911(0.438)$ \\
\hline & $A R_{0.2}$ & $0.807(0.427)$ & $0.817(0.426)$ & $0.748(0.392)$ & $0.837(0.427)$ \\
\hline & $A R_{0.5}$ & $0.646(0.415)$ & $0.540(0.372)$ & $0.594(0.400)$ & $0.540(0.372)$ \\
\hline & $A R_{-0.5}$ & $0.980(0.318)$ & $0.995(0.383)$ & $0.940(0.278)$ & $0.998(0.384)$ \\
\hline & $M A_{0.2}$ & $0.839(0.428)$ & $0.853(0.428)$ & $0.738(0.389)$ & $0.838(0.427)$ \\
\hline
\end{tabular}

Table 2.19: Simultaneous coverage probabilities (average median interval length) over $G_{\text {sub }}$ for $k=3$ and $a=0.3$. 
2.C Additional simulation Results

\begin{tabular}{|c|c|c|c|c|c|}
\hline \multirow[b]{2}{*}{$h$} & \multirow[b]{2}{*}{ DGP } & \multirow{2}{*}{$\frac{-}{\text { SWB }}$} & \multirow{2}{*}{$\begin{array}{c}\gamma=0 \\
\mathrm{WB}\end{array}$} & \multicolumn{2}{|c|}{$\gamma=0.2$} \\
\hline & & & & AWB & DWB \\
\hline \multirow{5}{*}{0.02} & 0 & $0.861(0.525)$ & $0.906(0.532)$ & $0.916(0.520)$ & $0.904(0.533)$ \\
\hline & $A R_{0.2}$ & $0.687(0.504)$ & $0.815(0.517)$ & $0.896(0.519)$ & $0.848(0.518)$ \\
\hline & $A R_{0.5}$ & $0.238(0.393)$ & $0.567(0.439)$ & $0.679(0.457)$ & $0.585(0.439)$ \\
\hline & $A R_{-0.5}$ & $0.968(0.405)$ & $0.979(0.455)$ & $0.980(0.416)$ & $0.978(0.453)$ \\
\hline & $M A_{0.2}$ & $0.708(0.504)$ & $0.826(0.517)$ & $0.880(0.518)$ & $0.843(0.518)$ \\
\hline \multirow{5}{*}{0.04} & 0 & $0.856(0.389)$ & $0.897(0.398)$ & $0.901(0.392)$ & $0.901(0.398)$ \\
\hline & $A R_{0.2}$ & $0.665(0.373)$ & $0.766(0.388)$ & $0.821(0.394)$ & $0.782(0.388)$ \\
\hline & $A R_{0.5}$ & $0.150(0.291)$ & $0.389(0.335)$ & $0.495(0.358)$ & $0.404(0.335)$ \\
\hline & $A R_{-0.5}$ & $0.982(0.301)$ & $0.992(0.345)$ & $0.985(0.316)$ & $0.985(0.344)$ \\
\hline & $M A_{0.2}$ & $0.689(0.373)$ & $0.800(0.388)$ & $0.812(0.394)$ & $0.791(0.389)$ \\
\hline \multirow{5}{*}{0.06} & 0 & $0.853(0.325)$ & $0.901(0$ & $0.899(0.335)$ & $0.907(0.337)$ \\
\hline & $A R_{0.2}$ & $0.686(0.312)$ & $0.750(0.329)$ & $0.798(0.338)$ & $0.779(0.330)$ \\
\hline & $A R_{0.5}$ & $0.161(0.244)$ & $0.372(0.288)$ & $0.443(0.311)$ & $0.354(0.288)$ \\
\hline & $A R_{-0.5}$ & $0.994(0.252)$ & $0.998(0.296)$ & $0.996(0.273)$ & $0.995(0.295)$ \\
\hline & $M A_{0.2}$ & $0.719(0.311)$ & $0.789(0.329)$ & $0.804(0.337)$ & $0.793(0.330)$ \\
\hline \multirow[b]{2}{*}{$h$} & & \multicolumn{2}{|c|}{$\gamma=0.4$} & \multicolumn{2}{|c|}{$\gamma=0.6$} \\
\hline & DGP & AWB & DWB & AWB & DWB \\
\hline \multirow{5}{*}{0.02} & 0 & $0.903(0.477)$ & $0.915(0.5$ & $0.810(0.402)$ & $0.921(0.533)$ \\
\hline & $A R_{0.2}$ & $0.859(0.487)$ & $0.824(0.514)$ & $0.738(0.423)$ & $0.830(0.516)$ \\
\hline & $A R_{0.5}$ & $0.650(0.450)$ & $0.544(0.436)$ & $0.554(0.411)$ & $0.564(0.438)$ \\
\hline & $A R_{-0.5}$ & $0.958(0.359)$ & $0.972(0.452)$ & $0.902(0.286)$ & $0.977(0.454)$ \\
\hline & $M A_{0.2}$ & $0.864(0.487)$ & $0.848(0.518)$ & $0.765(0.421)$ & $0.859(0.518)$ \\
\hline \multirow{5}{*}{0.04} & 0 & $0.864(0.366)$ & $0.908(0$ & $0.727(0.317)$ & $0.904(0.397)$ \\
\hline & $A R_{0.2}$ & $0.767(0.378)$ & $0.763(0.386)$ & $0.659(0.338)$ & $0.763(0.386)$ \\
\hline & $A R_{0.5}$ & $0.497(0.362)$ & $0.384(0.333)$ & $0.453(0.341)$ & $0.407(0.333)$ \\
\hline & $A R_{-0.5}$ & $0.956(0.277)$ & $0.989(0.344)$ & $0.896(0.229)$ & $0.993(0.345)$ \\
\hline & $M A_{0.2}$ & $0.789(0.378)$ & $0.806(0.387)$ & $0.661(0.336)$ & $0.789(0.388)$ \\
\hline \multirow{5}{*}{0.06} & 0 & $0.853(0.317)$ & $0.901(0.337)$ & $0.733(0.284)$ & $0.898(0.336)$ \\
\hline & $A R_{0.2}$ & $0.739(0.329)$ & $0.753(0.327)$ & $0.633(0.302)$ & $0.760(0.328)$ \\
\hline & $A R_{0.5}$ & $0.490(0.320)$ & $0.375(0.286)$ & $0.430(0.309)$ & $0.385(0.285)$ \\
\hline & $A R_{-}$ & $0.979(0.244)$ & $0.997(0.295)$ & $0.943(0.214)$ & $0.997(0.295)$ \\
\hline & $M A_{0.2}$ & $0.766(0.329)$ & $0.777(0.329)$ & $0.652(0.300)$ & $0.777(0.328)$ \\
\hline
\end{tabular}

Table 2.20: Simultaneous coverage probabilities (average median interval length) over $G$ for $k=3$ and $a=0.3$. 


\begin{tabular}{|c|c|c|c|c|c|}
\hline \multirow[b]{2}{*}{$h$} & \multirow[b]{2}{*}{ DGP } & \multirow{2}{*}{$\frac{-}{\text { SWB }}$} & \multirow{2}{*}{$\begin{array}{c}\gamma=0 \\
\mathrm{WB}\end{array}$} & \multicolumn{2}{|c|}{$\gamma=0.2$} \\
\hline & & & & AWB & DWB \\
\hline \multirow{5}{*}{0.02} & 0 & $0.923(0.707)$ & $0.946(0.722)$ & $0.952(0.706)$ & $0.946(0.723)$ \\
\hline & $A R_{0.2}$ & $0.859(0.679)$ & $0.902(0.702)$ & $0.919(0.703)$ & $0.902(0.703)$ \\
\hline & $A R_{0.5}$ & $0.672(0.530)$ & $0.780(0.594)$ & $0.819(0.619)$ & $0.778(0.594)$ \\
\hline & $A R_{-0.5}$ & $0.958(0.543)$ & $0.989(0.616)$ & $0.988(0.565)$ & $0.989(0.614)$ \\
\hline & $M A_{0.2}$ & $0.870(0.678)$ & $0.911(0.702)$ & $0.925(0.703)$ & $0.911(0.703)$ \\
\hline \multirow{5}{*}{0.04} & 0 & $0.920(0.534)$ & $0.950(0.552)$ & $0.950(0.545)$ & $0.951(0.553)$ \\
\hline & $A R_{0.2}$ & $0.859(0.513)$ & $0.901(0.539)$ & $0.911(0.547)$ & $0.900(0.538)$ \\
\hline & $A R_{0.5}$ & $0.664(0.401)$ & $0.766(0.466)$ & $0.805(0.496)$ & $0.769(0.465)$ \\
\hline & $A R_{-0.5}$ & $0.969(0.412)$ & $0.996(0.479)$ & $0.993(0.440)$ & $0.995(0.477)$ \\
\hline & $M A_{0.2}$ & $0.871(0.512)$ & $0.913(0.539)$ & $0.920(0.547)$ & $0.911(0.540)$ \\
\hline \multirow{5}{*}{0.06} & 0 & $0.924(0.451)$ & $0.956(0.473)$ & $0.955(0.471)$ & $0.956(0.474)$ \\
\hline & $A R_{0.2}$ & $0.863(0.433)$ & $0.908(0.463)$ & $0.919(0.475)$ & $0.909(0.463)$ \\
\hline & $A R_{0.5}$ & $0.665(0.339)$ & $0.780(0.404)$ & $0.816(0.437)$ & $0.782(0.405)$ \\
\hline & $A R_{-0.5}$ & $0.979(0.350)$ & $0.997(0.414)$ & $0.995(0.384)$ & $0.997(0.413)$ \\
\hline & $M A_{0.2}$ & $0.875(0.432)$ & $0.920(0.463)$ & $0.927(0.474)$ & $0.918(0.463)$ \\
\hline \multirow[b]{2}{*}{$h$} & \multirow[b]{2}{*}{ DGP } & \multicolumn{2}{|c|}{$\gamma=0.4$} & \multicolumn{2}{|c|}{$\gamma=0.6$} \\
\hline & & AWB & DWB & AWB & DWB \\
\hline \multirow{5}{*}{0.02} & 0 & $0.942(0.648)$ & $0.945(0.7$ & $0.905(0.546)$ & $0.947(0.724)$ \\
\hline & $A R_{0.2}$ & $0.912(0.659)$ & $0.903(0.699)$ & $0.867(0.573)$ & $0.901(0.700)$ \\
\hline & $A R_{0.5}$ & $0.828(0.608)$ & $0.780(0.592)$ & $0.790(0.554)$ & $0.778(0.593)$ \\
\hline & $A R_{-0.5}$ & $0.982(0.490)$ & $0.989(0.612)$ & $0.958(0.390)$ & $0.989(0.615)$ \\
\hline & $M A_{0.2}$ & $0.918(0.659)$ & $0.908(0.702)$ & $0.879(0.570)$ & $0.912(0.703)$ \\
\hline \multirow{5}{*}{0.04} & 0 & $0.938(0.509)$ & $0.948(0.553)$ & $0.902(0.441)$ & $0.951(0.552)$ \\
\hline & $A R_{0.2}$ & $0.904(0.524)$ & $0.901(0.535)$ & $0.865(0.469)$ & $0.898(0.536)$ \\
\hline & $A R_{0.5}$ & $0.817(0.501)$ & $0.767(0.463)$ & $0.793(0.471)$ & $0.765(0.462)$ \\
\hline & $A R_{-0.5}$ & $0.988(0.387)$ & $0.995(0.477)$ & $0.967(0.319)$ & $0.995(0.478)$ \\
\hline & $M A_{0.2}$ & $0.910(0.524)$ & $0.909(0.539)$ & $0.877(0.466)$ & $0.911(0.537)$ \\
\hline \multirow{5}{*}{0.06} & 0 & $0.943(0.446)$ & $0.953(0.474)$ & $0.914(0.397)$ & $0.955(0.472)$ \\
\hline & $A R_{0.2}$ & $0.911(0.462)$ & $0.906(0.460)$ & $0.880(0.423)$ & $0.904(0.461)$ \\
\hline & $A R_{0.5}$ & $0.828(0.447)$ & $0.778(0.402)$ & $0.815(0.431)$ & $0.777(0.402)$ \\
\hline & $A R_{-0.5}$ & $0.992(0.344)$ & $0.997(0.413)$ & $0.979(0.298)$ & $0.997(0.414)$ \\
\hline & $M A_{0.2}$ & $0.918(0.462)$ & $0.914(0.463)$ & $0.891(0.420)$ & $0.917(0.461)$ \\
\hline
\end{tabular}

Table 2.21: Pointwise coverage probabilities (average median interval length) for $k=3$ and $a=0.5$. 


\begin{tabular}{|c|c|c|c|c|c|}
\hline \multirow[b]{2}{*}{$h$} & \multirow[b]{2}{*}{ DGP } & \multirow{2}{*}{$\frac{-}{\text { SWB }}$} & \multirow{2}{*}{$\begin{array}{c}\gamma=0 \\
\mathrm{WB}\end{array}$} & \multicolumn{2}{|c|}{$\gamma=0.2$} \\
\hline & & & & AWB & DWB \\
\hline \multirow{5}{*}{0.02} & 0 & $0.884(0.653)$ & $0.896(0.667)$ & $0.905(0.651)$ & $0.911(0.668)$ \\
\hline & $A R_{0.2}$ & $0.785(0.627)$ & $0.827(0.648)$ & $0.869(0.649)$ & $0.840(0.650)$ \\
\hline & $A R_{0.5}$ & $0.417(0.489)$ & $0.649(0.549)$ & $0.695(0.571)$ & $0.639(0.549)$ \\
\hline & $A R_{-0.5}$ & $0.977(0.502)$ & $0.986(0.569)$ & $0.981(0.522)$ & $0.979(0.567)$ \\
\hline & $M A_{0.2}$ & $0.785(0.626)$ & $0.841(0.648)$ & $0.872(0.649)$ & $0.836(0.649)$ \\
\hline \multirow{5}{*}{0.04} & 0 & $0.891(0.496)$ & $0.912(0.513)$ & $0.906(0.506)$ & $0.917(0.513)$ \\
\hline & $A R_{0.2}$ & $0.753(0.476)$ & $0.805(0.500)$ & $0.849(0.508)$ & $0.839(0.500)$ \\
\hline & $A R_{0.5}$ & $0.339(0.372)$ & $0.570(0.432)$ & $0.631(0.461)$ & $0.565(0.432)$ \\
\hline & $A R_{-0.5}$ & $0.988(0.383)$ & $0.994(0.445)$ & $0.981(0.408)$ & $0.988(0.444)$ \\
\hline & $M A_{0.2}$ & $0.771(0.476)$ & $0.830(0.500)$ & $0.843(0.508)$ & $0.823(0.502)$ \\
\hline \multirow{5}{*}{0.06} & 0 & $0.883(0.420)$ & $0.919(0.441)$ & $0.920(0.438)$ & $0.922(0.441)$ \\
\hline & $A R_{0.2}$ & $0.775(0.404)$ & $0.818(0.431)$ & $0.839(0.442)$ & $0.834(0.431)$ \\
\hline & $A R_{0.5}$ & $0.337(0.315)$ & $0.543(0.376)$ & $0.609(0.406)$ & $0.543(0.377)$ \\
\hline & $A R_{-0.5}$ & $0.998(0.326)$ & $0.998(0.386)$ & $0.992(0.358)$ & $0.993(0.385)$ \\
\hline & $M A_{0.2}$ & $0.795(0.403)$ & $0.835(0.431)$ & $0.859(0.441)$ & $0.852(0.431)$ \\
\hline & \multirow[b]{2}{*}{ DGP } & \multicolumn{2}{|c|}{$\gamma=0.4$} & \multicolumn{2}{|c|}{$\gamma=0.6$} \\
\hline & & AWB & DWB & AWB & DWB \\
\hline \multirow{5}{*}{0.02} & 0 & $0.892(0.598)$ & $0.914(0.668)$ & $0.784(0.504)$ & $0.911(0.668)$ \\
\hline & $A R_{0.2}$ & $0.826(0.608)$ & $0.836(0.646)$ & $0.754(0.528)$ & $0.853(0.647)$ \\
\hline & $A R_{0.5}$ & $0.687(0.561)$ & $0.637(0.547)$ & $0.592(0.511)$ & $0.628(0.548)$ \\
\hline & $A R_{-0.5}$ & $0.958(0.452)$ & $0.974(0.565)$ & $0.900(0.360)$ & $0.980(0.568)$ \\
\hline & $M A_{0.2}$ & $0.845(0.608)$ & $0.859(0.649)$ & $0.750(0.526)$ & $0.860(0.648)$ \\
\hline \multirow{5}{*}{0.04} & 0 & $0.881(0.472)$ & $0.925(0.514)$ & $0.763(0.408)$ & $0.906(0.512)$ \\
\hline & $A R_{0.2}$ & $0.795(0.487)$ & $0.810(0.498)$ & $0.735(0.434)$ & $0.838(0.499)$ \\
\hline & $A R_{0.5}$ & $0.629(0.465)$ & $0.557(0.429)$ & $0.571(0.437)$ & $0.552(0.429)$ \\
\hline & $A R_{-0.5}$ & $0.966(0.358)$ & $0.988(0.443)$ & $0.923(0.296)$ & $0.988(0.445)$ \\
\hline & $M A_{0.2}$ & $0.836(0.486)$ & $0.858(0.500)$ & $0.713(0.431)$ & $0.836(0.499)$ \\
\hline \multirow{5}{*}{0.06} & 0 & $0.901(0.415)$ & $0.933(0.441)$ & $0.788(0.369)$ & $0.912(0.440)$ \\
\hline & $A R_{0.2}$ & $0.802(0.430)$ & $0.812(0.429)$ & $0.742(0.394)$ & $0.839(0.429)$ \\
\hline & $A R_{0.5}$ & $0.653(0.416)$ & $0.551(0.374)$ & $0.602(0.400)$ & $0.559(0.374)$ \\
\hline & $A R_{-0.5}$ & $0.980(0.320)$ & $0.994(0.385)$ & $0.947(0.276)$ & $0.998(0.385)$ \\
\hline & $M A_{0.2}$ & $0.842(0.430)$ & $0.860(0.431)$ & $0.742(0.390)$ & $0.834(0.430)$ \\
\hline
\end{tabular}

Table 2.22: Simultaneous coverage probabilities (average median interval length) over $G_{\text {sub }}$ for $k=3$ and $a=0.5$. 


\begin{tabular}{|c|c|c|c|c|c|}
\hline \multirow[b]{2}{*}{$h$} & \multirow[b]{2}{*}{ DGP } & \multirow{2}{*}{$\frac{-}{\mathrm{SWB}}$} & \multirow{2}{*}{$\begin{array}{c}\gamma=0 \\
\mathrm{WB}\end{array}$} & \multicolumn{2}{|c|}{$\gamma=0.2$} \\
\hline & & & & AWB & DWB \\
\hline \multirow{5}{*}{0.02} & 0 & $0.862(0.516)$ & $0.901(0.527)$ & $0.910(0.515)$ & $0.898(0.528)$ \\
\hline & $A R_{0.2}$ & $0.686(0.496)$ & $0.802(0.512)$ & $0.877(0.514)$ & $0.840(0.514)$ \\
\hline & $A R_{0.5}$ & $0.250(0.387)$ & $0.560(0.434)$ & $0.664(0.453)$ & $0.567(0.434)$ \\
\hline & $A R_{-0.5}$ & $0.967(0.397)$ & $0.979(0.450)$ & $0.979(0.413)$ & $0.978(0.448)$ \\
\hline & $M A_{0.2}$ & $0.698(0.496)$ & $0.824(0.513)$ & $0.870(0.513)$ & $0.832(0.514)$ \\
\hline \multirow{5}{*}{0.04} & 0 & $0.852(0.383)$ & $0.891(0.397)$ & $0.900(0.391)$ & $0.903(0.397)$ \\
\hline & $A R_{0.2}$ & $0.666(0.369)$ & $0.759(0.387)$ & $0.803(0.393)$ & $0.783(0.387)$ \\
\hline & $A R_{0.5}$ & $0.155(0.288)$ & $0.396(0.334)$ & $0.492(0.357)$ & $0.401(0.334)$ \\
\hline & $A R_{-0.5}$ & $0.988(0.296)$ & $0.992(0.344)$ & $0.980(0.316)$ & $0.985(0.343)$ \\
\hline & $M A_{0.2}$ & $0.689(0.368)$ & $0.793(0.387)$ & $0.814(0.394)$ & $0.786(0.388)$ \\
\hline \multirow{5}{*}{0.06} & 0 & $0.856(0.322)$ & $0.902(0.337)$ & $0.900(0.336)$ & $0.908(0.338)$ \\
\hline & $A R_{0.2}$ & $0.679(0.309)$ & $0.752(0.331)$ & $0.802(0.339)$ & $0.789(0.330)$ \\
\hline & $A R_{0.5}$ & $0.158(0.241)$ & $0.364(0.288)$ & $0.459(0.312)$ & $0.360(0.289)$ \\
\hline & $A R_{-0.5}$ & $0.994(0.250)$ & $0.997(0.296)$ & $0.992(0.274)$ & $0.995(0.295)$ \\
\hline & $M A_{0.2}$ & $0.721(0.308)$ & $0.795(0.330)$ & $0.812(0.339)$ & $0.797(0.331)$ \\
\hline & \multirow[b]{2}{*}{ DGP } & \multicolumn{2}{|c|}{$\gamma=0.4$} & \multicolumn{2}{|c|}{$\gamma=0.6$} \\
\hline & & AWB & DWB & AWB & DWB \\
\hline \multirow{5}{*}{0.02} & 0 & $0.896(0.473)$ & $0.913(0.528)$ & $0.794(0.399)$ & $0.911(0.528)$ \\
\hline & $A R_{0.2}$ & $0.836(0.482)$ & $0.814(0.510)$ & $0.722(0.419)$ & $0.822(0.511)$ \\
\hline & $A R_{0.5}$ & $0.633(0.445)$ & $0.533(0.432)$ & $0.538(0.407)$ & $0.554(0.433)$ \\
\hline & $A R_{-0.5}$ & $0.954(0.357)$ & $0.973(0.446)$ & $0.901(0.284)$ & $0.978(0.450)$ \\
\hline & $M A_{0.2}$ & $0.849(0.482)$ & $0.842(0.513)$ & $0.740(0.418)$ & $0.848(0.513)$ \\
\hline \multirow{5}{*}{0.04} & 0 & $0.859(0.365)$ & $0.902(0.397)$ & $0.725(0.317)$ & $0.900(0.396)$ \\
\hline & $A R_{0.2}$ & $0.758(0.377)$ & $0.755(0.385)$ & $0.648(0.337)$ & $0.768(0.385)$ \\
\hline & $A R_{0.5}$ & $0.501(0.360)$ & $0.388(0.332)$ & $0.448(0.339)$ & $0.398(0.332)$ \\
\hline & $A R_{-0.5}$ & $0.958(0.277)$ & $0.987(0.343)$ & $0.902(0.229)$ & $0.993(0.344)$ \\
\hline & $M A_{0.2}$ & $0.786(0.377)$ & $0.804(0.387)$ & $0.663(0.335)$ & $0.793(0.386)$ \\
\hline \multirow{5}{*}{0.06} & 0 & $0.855(0.319)$ & $0.906(0.338)$ & $0.731(0.284)$ & $0.895(0.337)$ \\
\hline & $A R_{0.2}$ & $0.747(0.330)$ & $0.755(0.329)$ & $0.627(0.303)$ & $0.763(0.329)$ \\
\hline & $A R_{0.5}$ & $0.488(0.320)$ & $0.387(0.287)$ & $0.432(0.308)$ & $0.398(0.286)$ \\
\hline & $A R_{-0.5}$ & $0.978(0.245)$ & $0.995(0.295)$ & $0.950(0.213)$ & $0.997(0.295)$ \\
\hline & $M A_{0.2}$ & $0.770(0.330)$ & $0.781(0.330)$ & $0.657(0.300)$ & $0.774(0.329)$ \\
\hline
\end{tabular}

Table 2.23: Simultaneous coverage probabilities (average median interval length) over $G$ for $k=3$ and $a=0.5$. 
2.C Additional simulation Results

\begin{tabular}{|c|c|c|c|c|c|}
\hline \multirow[b]{2}{*}{$h$} & \multirow[b]{2}{*}{ DGP } & \multirow{2}{*}{$\frac{-}{\text { SWB }}$} & \multirow{2}{*}{$\begin{array}{c}\gamma=0 \\
\mathrm{WB}\end{array}$} & \multicolumn{2}{|c|}{$\gamma=0.2$} \\
\hline & & & & AWB & DWB \\
\hline \multirow{5}{*}{0.02} & 0 & $0.920(0.691)$ & $0.946(0.713)$ & $0.951(0.699)$ & $0.946(0.716)$ \\
\hline & $A R_{0.2}$ & $0.856(0.665)$ & $0.901(0.694)$ & $0.918(0.696)$ & $0.902(0.695)$ \\
\hline & $A R_{0.5}$ & $0.670(0.518)$ & $0.783(0.589)$ & $0.819(0.613)$ & $0.779(0.589)$ \\
\hline & $A R_{-0.5}$ & $0.956(0.530)$ & $0.989(0.608)$ & $0.988(0.559)$ & $0.989(0.606)$ \\
\hline & $M A_{0.2}$ & $0.867(0.663)$ & $0.911(0.694)$ & $0.924(0.697)$ & $0.910(0.695)$ \\
\hline \multirow{5}{*}{0.04} & 0 & $0.918(0.523)$ & $0.951(0.552)$ & $0.951(0.545)$ & $0.951(0.552)$ \\
\hline & $A R_{0.2}$ & $0.857(0.505)$ & $0.902(0.539)$ & $0.912(0.547)$ & $0.901(0.538)$ \\
\hline & $A R_{0.5}$ & $0.662(0.394)$ & $0.768(0.465)$ & $0.806(0.495)$ & $0.771(0.465)$ \\
\hline & $A R_{-0.5}$ & $0.967(0.405)$ & $0.995(0.478)$ & $0.993(0.441)$ & $0.995(0.477)$ \\
\hline & $M A_{0.2}$ & $0.869(0.503)$ & $0.913(0.538)$ & $0.920(0.547)$ & $0.911(0.539)$ \\
\hline \multirow{5}{*}{0.06} & 0 & $0.922(0.445)$ & $0.957(0.476)$ & $0.956(0.474)$ & $0.956(0.477)$ \\
\hline & $A R_{0.2}$ & $0.860(0.427)$ & $0.909(0.466)$ & $0.920(0.479)$ & $0.910(0.466)$ \\
\hline & $A R_{0.5}$ & $0.662(0.333)$ & $0.783(0.407)$ & $0.819(0.439)$ & $0.785(0.408)$ \\
\hline & $A R_{-0.5}$ & $0.976(0.345)$ & $0.997(0.415)$ & $0.996(0.388)$ & $0.997(0.416)$ \\
\hline & $M A_{0.2}$ & $0.873(0.427)$ & $0.921(0.466)$ & $0.928(0.477)$ & $0.920(0.466)$ \\
\hline \multirow[b]{2}{*}{$h$} & & \multicolumn{2}{|c|}{$\gamma=0.4$} & \multicolumn{2}{|c|}{$\gamma=0.6$} \\
\hline & DGP & AWB & DWB & AWB & DWB \\
\hline \multirow{5}{*}{0.02} & 0 & $0.941(0.642)$ & $0.945(0.715)$ & $0.903(0.541)$ & $0.947(0.714)$ \\
\hline & $A R_{0.2}$ & $0.911(0.653)$ & $0.903(0.692)$ & $0.866(0.568)$ & $0.901(0.693)$ \\
\hline & $A R_{0.5}$ & $0.827(0.602)$ & $0.781(0.586)$ & $0.790(0.547)$ & $0.780(0.588)$ \\
\hline & $A R_{-0.5}$ & $0.982(0.487)$ & $0.989(0.603)$ & $0.957(0.389)$ & $0.989(0.607)$ \\
\hline & $M A_{0.2}$ & $0.916(0.652)$ & $0.908(0.695)$ & $0.878(0.565)$ & $0.911(0.694)$ \\
\hline \multirow{5}{*}{0.04} & 0 & $0.938(0.509)$ & $0.949(0.552)$ & $0.903(0.442)$ & $0.951(0.551)$ \\
\hline & $A R_{0.2}$ & $0.904(0.524)$ & $0.901(0.536)$ & $0.866(0.468)$ & $0.898(0.535)$ \\
\hline & $A R_{0.5}$ & $0.819(0.499)$ & $0.770(0.462)$ & $0.795(0.470)$ & $0.767(0.462)$ \\
\hline & $A R_{-0.5}$ & $0.988(0.389)$ & $0.995(0.476)$ & $0.967(0.322)$ & $0.995(0.477)$ \\
\hline & $M A_{0.2}$ & $0.911(0.525)$ & $0.910(0.538)$ & $0.878(0.466)$ & $0.912(0.537)$ \\
\hline \multirow{5}{*}{0.06} & 0 & $0.944(0.451)$ & $0.953(0.476)$ & $0.916(0.401)$ & $0.955(0.475)$ \\
\hline & $A R_{0.2}$ & $0.913(0.464)$ & $0.907(0.464)$ & $0.883(0.426)$ & $0.905(0.464)$ \\
\hline & $A R_{0.5}$ & $0.831(0.449)$ & $0.781(0.405)$ & $0.818(0.432)$ & $0.780(0.405)$ \\
\hline & $A R_{-0.5}$ & $0.992(0.348)$ & $0.997(0.415)$ & $0.980(0.301)$ & $0.997(0.416)$ \\
\hline & $M A_{0.2}$ & $0.920(0.465)$ & $0.915(0.465)$ & $0.893(0.423)$ & $0.918(0.464)$ \\
\hline
\end{tabular}

Table 2.24: Pointwise coverage probabilities (average median interval length) for $k=3$ and $a=0.7$. 


\begin{tabular}{|c|c|c|c|c|c|}
\hline \multirow[b]{2}{*}{$h$} & \multirow[b]{2}{*}{ DGP } & \multirow{2}{*}{$\frac{-}{\mathrm{SWB}}$} & \multirow{2}{*}{$\begin{array}{c}\gamma=0 \\
\mathrm{WB}\end{array}$} & \multicolumn{2}{|c|}{$\gamma=0.2$} \\
\hline & & & & AWB & DWB \\
\hline \multirow{5}{*}{0.02} & 0 & $0.874(0.639)$ & $0.891(0.659)$ & $0.894(0.645)$ & $0.895(0.660)$ \\
\hline & $A R_{0.2}$ & $0.787(0.614)$ & $0.824(0.642)$ & $0.845(0.642)$ & $0.832(0.643)$ \\
\hline & $A R_{0.5}$ & $0.432(0.479)$ & $0.648(0.544)$ & $0.672(0.565)$ & $0.636(0.544)$ \\
\hline & $A R_{-0.5}$ & $0.981(0.489)$ & $0.983(0.562)$ & $0.978(0.517)$ & $0.979(0.560)$ \\
\hline & $M A_{0.2}$ & $0.777(0.613)$ & $0.832(0.640)$ & $0.853(0.643)$ & $0.826(0.643)$ \\
\hline \multirow{5}{*}{0.04} & 0 & $0.899(0.487)$ & $0.909(0.513)$ & $0.910(0.507)$ & $0.915(0.514)$ \\
\hline & $A R_{0.2}$ & $0.764(0.469)$ & $0.807(0.501)$ & $0.850(0.508)$ & $0.847(0.500)$ \\
\hline & $A R_{0.5}$ & $0.343(0.366)$ & $0.581(0.432)$ & $0.629(0.461)$ & $0.574(0.433)$ \\
\hline & $A R_{-0.5}$ & $0.989(0.377)$ & $0.991(0.445)$ & $0.984(0.410)$ & $0.988(0.443)$ \\
\hline & $M A_{0.2}$ & $0.771(0.468)$ & $0.832(0.500)$ & $0.844(0.509)$ & $0.828(0.501)$ \\
\hline \multirow{5}{*}{0.06} & 0 & $0.897(0.415)$ & $0.920(0.445)$ & $0.920(0.443)$ & $0.928(0.445)$ \\
\hline & $A R_{0.2}$ & $0.777(0.399)$ & $0.823(0.434)$ & $0.846(0.446)$ & $0.841(0.435)$ \\
\hline & $A R_{0.5}$ & $0.345(0.311)$ & $0.566(0.379)$ & $0.633(0.409)$ & $0.552(0.380)$ \\
\hline & $A R_{-0.5}$ & $0.996(0.321)$ & $0.998(0.388)$ & $0.990(0.362)$ & $0.991(0.388)$ \\
\hline & $M A_{0.2}$ & $0.807(0.398)$ & $0.839(0.434)$ & $0.869(0.446)$ & $0.858(0.435)$ \\
\hline & \multirow[b]{2}{*}{ DGP } & \multicolumn{2}{|c|}{$\gamma=0.4$} & \multicolumn{2}{|c|}{$\gamma=0.6$} \\
\hline & & AWB & DWB & AWB & DWB \\
\hline \multirow{5}{*}{0.02} & 0 & $0.869(0.592)$ & $0.908(0.660)$ & $0.764(0.499)$ & $0.902(0.660)$ \\
\hline & $A R_{0.2}$ & $0.805(0.603)$ & $0.818(0.639)$ & $0.723(0.523)$ & $0.846(0.640)$ \\
\hline & $A R_{0.5}$ & $0.666(0.554)$ & $0.629(0.542)$ & $0.584(0.504)$ & $0.633(0.543)$ \\
\hline & $A R_{-0.5}$ & $0.952(0.450)$ & $0.976(0.558)$ & $0.891(0.359)$ & $0.978(0.561)$ \\
\hline & $M A_{0.2}$ & $0.821(0.602)$ & $0.845(0.642)$ & $0.723(0.520)$ & $0.847(0.641)$ \\
\hline \multirow{5}{*}{0.04} & 0 & $0.875(0.474)$ & $0.927(0.513)$ & $0.762(0.410)$ & $0.908(0.512)$ \\
\hline & $A R_{0.2}$ & $0.783(0.487)$ & $0.805(0.498)$ & $0.729(0.435)$ & $0.838(0.498)$ \\
\hline & $A R_{0.5}$ & $0.632(0.463)$ & $0.571(0.429)$ & $0.576(0.435)$ & $0.566(0.430)$ \\
\hline & $A R_{-0.5}$ & $0.969(0.361)$ & $0.986(0.442)$ & $0.929(0.299)$ & $0.989(0.444)$ \\
\hline & $M A_{0.2}$ & $0.832(0.487)$ & $0.855(0.500)$ & $0.714(0.432)$ & $0.840(0.499)$ \\
\hline \multirow{5}{*}{0.06} & 0 & $0.908(0.420)$ & $0.942(0.444)$ & $0.811(0.373)$ & $0.916(0.444)$ \\
\hline & $A R_{0.2}$ & $0.811(0.433)$ & $0.821(0.432)$ & $0.763(0.397)$ & $0.848(0.433)$ \\
\hline & $A R_{0.5}$ & $0.670(0.418)$ & $0.558(0.377)$ & $0.624(0.401)$ & $0.582(0.377)$ \\
\hline & $A R_{-0.5}$ & $0.983(0.324)$ & $0.997(0.387)$ & $0.956(0.279)$ & $0.999(0.388)$ \\
\hline & $M A_{0.2}$ & $0.854(0.434)$ & $0.868(0.434)$ & $0.763(0.393)$ & $0.837(0.433)$ \\
\hline
\end{tabular}

Table 2.25: Simultaneous coverage probabilities (average median interval length) over $G_{s u b}$ for $k=3$ and $a=0.7$. 
2.C Additional simulation Results

\begin{tabular}{|c|c|c|c|c|c|}
\hline \multirow[b]{2}{*}{$h$} & \multirow[b]{2}{*}{ DGP } & \multirow{2}{*}{$\frac{-}{\text { SWB }}$} & \multirow{2}{*}{$\begin{array}{c}\gamma=0 \\
\mathrm{WB}\end{array}$} & \multicolumn{2}{|c|}{$\gamma=0.2$} \\
\hline & & & & AWB & DWB \\
\hline \multirow{5}{*}{0.02} & 0 & $0.855(0.505)$ & $0.890(0.521)$ & $0.900(0.510)$ & $0.893(0.522)$ \\
\hline & $A R_{0.2}$ & $0.690(0.486)$ & $0.791(0.507)$ & $0.856(0.508)$ & $0.824(0.508)$ \\
\hline & $A R_{0.5}$ & $0.257(0.379)$ & $0.555(0.430)$ & $0.640(0.448)$ & $0.557(0.430)$ \\
\hline & $A R_{-0.5}$ & $0.969(0.387)$ & $0.978(0.444)$ & $0.980(0.408)$ & $0.976(0.443)$ \\
\hline & $M A_{0.2}$ & $0.694(0.485)$ & $0.816(0.507)$ & $0.858(0.509)$ & $0.819(0.508)$ \\
\hline \multirow{5}{*}{0.04} & 0 & $0.862(0.376)$ & $0.890(0.396)$ & $0.898(0.391)$ & $0.897(0.396)$ \\
\hline & $A R_{0.2}$ & $0.671(0.362)$ & $0.758(0.386)$ & $0.815(0.393)$ & $0.784(0.386)$ \\
\hline & $A R_{0.5}$ & $0.165(0.282)$ & $0.398(0.334)$ & $0.498(0.356)$ & $0.401(0.334)$ \\
\hline & $A R_{-0.5}$ & $0.990(0.291)$ & $0.993(0.343)$ & $0.982(0.316)$ & $0.988(0.342)$ \\
\hline & $M A_{0.2}$ & $0.691(0.361)$ & $0.788(0.386)$ & $0.814(0.393)$ & $0.793(0.387)$ \\
\hline \multirow{5}{*}{0.06} & 0 & $0.863(0.317)$ & $0.900(0.339)$ & $0.907(0.339)$ & $0.910(0.340)$ \\
\hline & $A R_{0.2}$ & $0.678(0.305)$ & $0.762(0.332)$ & $0.807(0.341)$ & $0.796(0.332)$ \\
\hline & $A R_{0.5}$ & $0.153(0.237)$ & $0.382(0.290)$ & $0.472(0.313)$ & $0.377(0.291)$ \\
\hline & $A R_{-0.5}$ & $0.994(0.245)$ & $0.997(0.296)$ & $0.993(0.277)$ & $0.997(0.297)$ \\
\hline & $M A_{0.2}$ & $0.725(0.304)$ & $0.794(0.332)$ & $0.822(0.341)$ & $0.798(0.332)$ \\
\hline \multirow[b]{2}{*}{$h$} & & \multicolumn{2}{|c|}{$\gamma=0.4$} & \multicolumn{2}{|c|}{$\gamma=0.6$} \\
\hline & DGP & AWB & DWB & AWB & DWB \\
\hline \multirow{5}{*}{0.02} & 0 & $0.880(0.468)$ & $0.906(0.522)$ & $0.770(0.396)$ & $0.906(0.522)$ \\
\hline & $A R_{0.2}$ & $0.809(0.478)$ & $0.789(0.505)$ & $0.699(0.415)$ & $0.818(0.506)$ \\
\hline & $A R_{0.5}$ & $0.617(0.440)$ & $0.531(0.428)$ & $0.508(0.402)$ & $0.542(0.429)$ \\
\hline & $A R_{-0.5}$ & $0.946(0.355)$ & $0.971(0.440)$ & $0.895(0.284)$ & $0.978(0.443)$ \\
\hline & $M A_{0.2}$ & $0.824(0.477)$ & $0.835(0.507)$ & $0.716(0.413)$ & $0.841(0.507)$ \\
\hline \multirow{5}{*}{0.04} & 0 & $0.854(0.366)$ & $0.898(0.396)$ & $0.722(0.318)$ & $0.902(0.395)$ \\
\hline & $A R_{0.2}$ & $0.756(0.377)$ & $0.761(0.385)$ & $0.644(0.337)$ & $0.759(0.384)$ \\
\hline & $A R_{0.5}$ & $0.492(0.359)$ & $0.398(0.332)$ & $0.452(0.338)$ & $0.409(0.332)$ \\
\hline & $A R_{-0.5}$ & $0.960(0.279)$ & $0.986(0.341)$ & $0.904(0.232)$ & $0.994(0.342)$ \\
\hline & $M A_{0.2}$ & $0.782(0.377)$ & $0.796(0.386)$ & $0.654(0.335)$ & $0.795(0.386)$ \\
\hline \multirow{5}{*}{0.06} & 0 & $0.862(0.322)$ & $0.909(0.340)$ & $0.748(0.286)$ & 0.905 \\
\hline & $A R_{0.2}$ & $0.752(0.332)$ & $0.744(0.331)$ & $0.650(0.305)$ & $0.764(0.330)$ \\
\hline & $A R_{0.5}$ & $0.497(0.321)$ & $0.399(0.289)$ & $0.452(0.308)$ & $0.409(0.288)$ \\
\hline & $A R_{-0.5}$ & $0.981(0.248)$ & $0.996(0.296)$ & $0.952(0.215)$ & $0.997(0.296)$ \\
\hline & $M A_{0.2}$ & $0.777(0.332)$ & $0.799(0.332)$ & $0.677(0.302)$ & $0.786(0.331)$ \\
\hline
\end{tabular}

Table 2.26: Simultaneous coverage probabilities (average median interval length) over $G$ for $k=3$ and $a=0.7$. 


\begin{tabular}{|c|c|c|c|c|c|}
\hline \multirow[b]{2}{*}{$h$} & \multirow[b]{2}{*}{ DGP } & \multirow{2}{*}{$\frac{-}{\text { SWB }}$} & \multirow{2}{*}{$\begin{array}{c}\gamma=0 \\
\mathrm{WB}\end{array}$} & \multicolumn{2}{|c|}{$\gamma=0.2$} \\
\hline & & & & AWB & DWB \\
\hline \multirow{5}{*}{0.02} & 0 & $0.928(0.722)$ & $0.946(0.731)$ & $0.952(0.713)$ & $0.946(0.731)$ \\
\hline & $A R_{0.2}$ & $0.863(0.691)$ & $0.902(0.710)$ & $0.919(0.710)$ & $0.903(0.710)$ \\
\hline & $A R_{0.5}$ & $0.674(0.539)$ & $0.780(0.604)$ & $0.820(0.628)$ & $0.779(0.601)$ \\
\hline & $A R_{-0.5}$ & $0.961(0.556)$ & $0.990(0.624)$ & $0.988(0.570)$ & $0.989(0.620)$ \\
\hline & $M A_{0.2}$ & $0.874(0.692)$ & $0.911(0.712)$ & $0.925(0.711)$ & $0.911(0.710)$ \\
\hline \multirow{5}{*}{0.04} & 0 & $0.924(0.545)$ & $0.950(0.559)$ & $0.951(0.551)$ & $0.951(0.559)$ \\
\hline & $A R_{0.2}$ & $0.864(0.521)$ & $0.901(0.543)$ & $0.912(0.553)$ & $0.901(0.545)$ \\
\hline & $A R_{0.5}$ & $0.669(0.408)$ & $0.767(0.471)$ & $0.806(0.503)$ & $0.771(0.471)$ \\
\hline & $A R_{-0.5}$ & $0.972(0.422)$ & $0.996(0.485)$ & $0.993(0.446)$ & $0.995(0.483)$ \\
\hline & $M A_{0.2}$ & $0.875(0.523)$ & $0.913(0.546)$ & $0.920(0.553)$ & $0.911(0.545)$ \\
\hline \multirow{5}{*}{0.06} & 0 & $0.928(0.459)$ & $0.956(0.480)$ & $0.955(0.479)$ & $0.955(0.481)$ \\
\hline & $A R_{0.2}$ & $0.867(0.439)$ & $0.908(0.468)$ & $0.919(0.482)$ & $0.909(0.469)$ \\
\hline & $A R_{0.5}$ & $0.669(0.344)$ & $0.780(0.411)$ & $0.817(0.443)$ & $0.783(0.410)$ \\
\hline & $A R_{-0.5}$ & $0.981(0.355)$ & $0.997(0.423)$ & $0.995(0.395)$ & $0.997(0.420)$ \\
\hline & $M A_{0.2}$ & $0.879(0.441)$ & $0.920(0.469)$ & $0.926(0.481)$ & $0.918(0.469)$ \\
\hline \multirow[b]{2}{*}{$h$} & \multirow[b]{2}{*}{ DGP } & \multicolumn{2}{|c|}{$\gamma=0.4$} & \multicolumn{2}{|c|}{$\gamma=0.6$} \\
\hline & & AWB & DWB & AWB & DWB \\
\hline \multirow{5}{*}{0.02} & 0 & $0.943(0.653)$ & $0.945(0.730)$ & $0.906(0.552)$ & $0.947(0.732)$ \\
\hline & $A R_{0.2}$ & $0.914(0.666)$ & $0.903(0.706)$ & $0.870(0.582)$ & $0.901(0.708)$ \\
\hline & $A R_{0.5}$ & $0.829(0.615)$ & $0.780(0.599)$ & $0.792(0.562)$ & $0.778(0.600)$ \\
\hline & $A R_{-0.5}$ & $0.982(0.494)$ & $0.989(0.621)$ & $0.959(0.393)$ & $0.990(0.623)$ \\
\hline & $M A_{0.2}$ & $0.919(0.666)$ & $0.908(0.710)$ & $0.880(0.578)$ & $0.912(0.711)$ \\
\hline \multirow{5}{*}{0.04} & 0 & $0.939(0.515)$ & $0.949(0.557)$ & $0.904(0.449)$ & $0.951(0.560)$ \\
\hline & $A R_{0.2}$ & $0.905(0.531)$ & $0.901(0.541)$ & $0.867(0.476)$ & $0.898(0.542)$ \\
\hline & $A R_{0.5}$ & $0.816(0.508)$ & $0.767(0.468)$ & $0.793(0.482)$ & $0.765(0.469)$ \\
\hline & $A R_{-0.5}$ & $0.988(0.394)$ & $0.995(0.484)$ & $0.969(0.327)$ & $0.995(0.485)$ \\
\hline & $M A_{0.2}$ & $0.911(0.530)$ & $0.909(0.543)$ & $0.878(0.474)$ & $0.912(0.545)$ \\
\hline \multirow{5}{*}{0.06} & 0 & $0.944(0.455)$ & $0.953(0.479)$ & $0.914(0.408)$ & $0.955(0.481)$ \\
\hline & $A R_{0.2}$ & $0.912(0.470)$ & $0.906(0.467)$ & $0.881(0.433)$ & $0.905(0.467)$ \\
\hline & $A R_{0.5}$ & $0.827(0.455)$ & $0.777(0.408)$ & $0.814(0.442)$ & $0.777(0.408)$ \\
\hline & $A R_{-0.5}$ & $0.992(0.359)$ & $0.997(0.422)$ & $0.978(0.314)$ & $0.997(0.423)$ \\
\hline & $M A_{0.2}$ & $0.918(0.470)$ & $0.914(0.467)$ & $0.891(0.430)$ & $0.917(0.469)$ \\
\hline
\end{tabular}

Table 2.27: Pointwise coverage probabilities (average median interval length) for $k=4$ and $a=0.3$. 
2.C Additional simulation Results

\begin{tabular}{|c|c|c|c|c|c|}
\hline \multirow[b]{2}{*}{$h$} & \multirow[b]{2}{*}{ DGP } & \multirow{2}{*}{$\frac{-}{\text { SWB }}$} & \multirow{2}{*}{$\begin{array}{c}\gamma=0 \\
\mathrm{WB}\end{array}$} & \multicolumn{2}{|c|}{$\gamma=0.2$} \\
\hline & & & & AWB & DWB \\
\hline \multirow{5}{*}{0.02} & 0 & $0.883(0.666)$ & $0.921(0.674)$ & $0.932(0.658)$ & $0.921(0.674)$ \\
\hline & $A R_{0.2}$ & $0.783(0.638)$ & $0.856(0.655)$ & $0.898(0.656)$ & $0.871(0.656)$ \\
\hline & $A R_{0.5}$ & $0.389(0.497)$ & $0.661(0.557)$ & $0.738(0.578)$ & $0.660(0.555)$ \\
\hline & $A R_{-0.5}$ & $0.982(0.513)$ & $0.984(0.575)$ & $0.978(0.526)$ & $0.979(0.573)$ \\
\hline & $M A_{0.2}$ & $0.786(0.638)$ & $0.869(0.656)$ & $0.893(0.656)$ & $0.860(0.656)$ \\
\hline \multirow{5}{*}{0.04} & 0 & $0.887(0.505)$ & $0.915(0.517)$ & $0.916(0.510)$ & $0.911(0.517)$ \\
\hline & $A R_{0.2}$ & $0.748(0.482)$ & $0.820(0.503)$ & $0.867(0.512)$ & $0.841(0.504)$ \\
\hline & $A R_{0.5}$ & $0.319(0.377)$ & $0.559(0.436)$ & $0.628(0.465)$ & $0.549(0.436)$ \\
\hline & $A R_{-0.5}$ & $0.988(0.391)$ & $0.991(0.449)$ & $0.984(0.413)$ & $0.985(0.447)$ \\
\hline & $M A_{0.2}$ & $0.763(0.484)$ & $0.834(0.505)$ & $0.861(0.513)$ & $0.828(0.505)$ \\
\hline \multirow{5}{*}{0.06} & 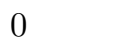 & $0.877(0.426)$ & $0.919(0.445)$ & $0.924(0.443)$ & 0.92 \\
\hline & $A R_{0.2}$ & $0.753(0.407)$ & $0.821(0.433)$ & $0.841(0.446)$ & $0.832(0.435)$ \\
\hline & $A R_{0.5}$ & $0.338(0.319)$ & $0.535(0.381)$ & $0.604(0.411)$ & $0.533(0.379)$ \\
\hline & $A R_{-0.5}$ & $0.997(0.329)$ & $0.997(0.392)$ & $0.990(0.366)$ & $0.992(0.389)$ \\
\hline & $M A_{0.2}$ & $0.794(0.409)$ & $0.837(0.434)$ & $0.859(0.446)$ & $0.853(0.435)$ \\
\hline \multirow[b]{2}{*}{$h$} & & \multicolumn{2}{|c|}{$\gamma=0.4$} & \multicolumn{2}{|c|}{$\gamma=0.6$} \\
\hline & DGP & AWB & DWB & AWB & DWB \\
\hline \multirow{5}{*}{0.02} & 0 & $0.926(0.602)$ & $0.923(0.6$ & $0.865(0.509)$ & $0.930(0.675)$ \\
\hline & $A R_{0.2}$ & $0.872(0.615)$ & $0.863(0.652)$ & $0.806(0.536)$ & $0.865(0.654)$ \\
\hline & $A R_{0.5}$ & $0.733(0.567)$ & $0.657(0.552)$ & $0.659(0.517)$ & $0.659(0.554)$ \\
\hline & $A R_{-0.5}$ & $0.961(0.456)$ & $0.977(0.574)$ & $0.909(0.363)$ & $0.980(0.574)$ \\
\hline & $M A_{0.2}$ & $0.893(0.615)$ & $0.875(0.655)$ & $0.807(0.533)$ & $0.886(0.655)$ \\
\hline \multirow{5}{*}{0.04} & 0 & $0.899(0.476)$ & $0.924(0$ & $0.786(0.415)$ & 0.918 \\
\hline & $A R_{0.2}$ & $0.826(0.492)$ & $0.821(0.501)$ & $0.767(0.440)$ & $0.837(0.502)$ \\
\hline & $A R_{0.5}$ & $0.634(0.470)$ & $0.544(0.434)$ & $0.581(0.446)$ & $0.559(0.434)$ \\
\hline & $A R_{-0.5}$ & $0.967(0.365)$ & $0.987(0.448)$ & $0.924(0.302)$ & $0.989(0.449)$ \\
\hline & $M A_{0.2}$ & $0.852(0.491)$ & $0.866(0.503)$ & $0.739(0.439)$ & $0.842(0.505)$ \\
\hline \multirow{5}{*}{0.06} & 0 & $0.897(0.422)$ & $0.929(0.444)$ & $0.786(0.377)$ & $0.920(0.445)$ \\
\hline & $A R_{0.2}$ & $0.807(0.435)$ & $0.817(0.432)$ & $0.744(0.400)$ & $0.837(0.433)$ \\
\hline & $A R_{0.5}$ & $0.645(0.422)$ & $0.530(0.378)$ & $0.597(0.408)$ & $0.530(0.378)$ \\
\hline & $A R_{-0}$ & $0.979(0.333)$ & $0.995(0.391)$ & $0.935(0.290)$ & $0.998(0.392)$ \\
\hline & $M A_{0.2}$ & $0.848(0.434)$ & $0.856(0.433)$ & $0.737(0.398)$ & $0.838(0.434)$ \\
\hline
\end{tabular}

Table 2.28: Simultaneous coverage probabilities (average median interval length) over $G_{\text {sub }}$ for $k=4$ and $a=0.3$. 


\begin{tabular}{|c|c|c|c|c|c|}
\hline \multirow[b]{2}{*}{$h$} & \multirow[b]{2}{*}{ DGP } & \multirow{2}{*}{$\frac{-}{\text { SWB }}$} & \multirow{2}{*}{$\begin{array}{c}\gamma=0 \\
\mathrm{WB}\end{array}$} & \multicolumn{2}{|c|}{$\gamma=0.2$} \\
\hline & & & & AWB & DWB \\
\hline \multirow{5}{*}{0.02} & 0 & $0.855(0.527)$ & $0.908(0.534)$ & $0.923(0.521)$ & $0.910(0.534)$ \\
\hline & $A R_{0.2}$ & $0.688(0.505)$ & $0.823(0.518)$ & $0.887(0.520)$ & $0.853(0.520)$ \\
\hline & $A R_{0.5}$ & $0.236(0.394)$ & $0.577(0.440)$ & $0.674(0.458)$ & $0.574(0.439)$ \\
\hline & $A R_{-0.5}$ & $0.980(0.406)$ & $0.979(0.455)$ & $0.978(0.416)$ & $0.977(0.453)$ \\
\hline & $M A_{0.2}$ & $0.711(0.506)$ & $0.837(0.520)$ & $0.876(0.519)$ & $0.848(0.519)$ \\
\hline \multirow{5}{*}{0.04} & 0 & $0.853(0.392)$ & $0.902(0.402)$ & $0.909(0.396)$ & $0.907(0.401)$ \\
\hline & $A R_{0.2}$ & $0.672(0.375)$ & $0.767(0.391)$ & $0.819(0.398)$ & $0.787(0.391)$ \\
\hline & $A R_{0.5}$ & $0.146(0.293)$ & $0.397(0.339)$ & $0.496(0.362)$ & $0.391(0.338)$ \\
\hline & $A R_{-0.5}$ & $0.985(0.303)$ & $0.994(0.349)$ & $0.987(0.320)$ & $0.983(0.347)$ \\
\hline & $M A_{0.2}$ & $0.693(0.376)$ & $0.806(0.392)$ & $0.826(0.398)$ & $0.796(0.392)$ \\
\hline \multirow{5}{*}{0.06} & 0 & $0.849(0.328)$ & $0.901(0.343)$ & $0.899(0.342)$ & $0.906(0.343)$ \\
\hline & $A R_{0.2}$ & $0.668(0.314)$ & $0.757(0.334)$ & $0.797(0.344)$ & $0.785(0.335)$ \\
\hline & $A R_{0.5}$ & $0.158(0.246)$ & $0.371(0.294)$ & $0.423(0.317)$ & $0.357(0.292)$ \\
\hline & $A R_{-0.5}$ & $0.995(0.254)$ & $0.996(0.302)$ & $0.993(0.282)$ & $0.994(0.300)$ \\
\hline & $M A_{0.2}$ & $0.712(0.315)$ & $0.805(0.335)$ & $0.813(0.344)$ & $0.803(0.335)$ \\
\hline & \multirow[b]{2}{*}{ DGP } & \multicolumn{2}{|c|}{$\gamma=0.4$} & \multicolumn{2}{|c|}{$\gamma=0.6$} \\
\hline & & AWB & DWB & AWB & DWB \\
\hline \multirow{5}{*}{0.02} & 0 & $0.913(0.477)$ & $0.915(0.533)$ & $0.850(0.404)$ & $0.925(0.534)$ \\
\hline & $A R_{0.2}$ & $0.874(0.487)$ & $0.831(0.516)$ & $0.746(0.425)$ & $0.815(0.518)$ \\
\hline & $A R_{0.5}$ & $0.640(0.450)$ & $0.563(0.437)$ & $0.562(0.412)$ & $0.573(0.438)$ \\
\hline & $A R_{-0.5}$ & $0.959(0.360)$ & $0.974(0.454)$ & $0.907(0.286)$ & $0.980(0.455)$ \\
\hline & $M A_{0.2}$ & $0.869(0.487)$ & $0.856(0.518)$ & $0.777(0.423)$ & $0.872(0.519)$ \\
\hline \multirow{5}{*}{0.04} & 0 & $0.858(0.369)$ & $0.908(0.400)$ & $0.728(0.322)$ & $0.909(0.402)$ \\
\hline & $A R_{0.2}$ & $0.767(0.382)$ & $0.762(0.389)$ & $0.657(0.343)$ & $0.757(0.390)$ \\
\hline & $A R_{0.5}$ & $0.505(0.366)$ & $0.396(0.336)$ & $0.465(0.347)$ & $0.401(0.337)$ \\
\hline & $A R_{-0.5}$ & $0.962(0.283)$ & $0.988(0.347)$ & $0.900(0.234)$ & $0.994(0.348)$ \\
\hline & $M A_{0.2}$ & $0.792(0.381)$ & $0.798(0.390)$ & $0.666(0.341)$ & $0.797(0.392)$ \\
\hline \multirow{5}{*}{0.06} & 0 & $0.850(0.325)$ & $0.900(0.342)$ & $0.731(0.291)$ & $0.897(0.343)$ \\
\hline & $A R_{0.2}$ & $0.732(0.336)$ & $0.752(0.333)$ & $0.618(0.309)$ & $0.761(0.334)$ \\
\hline & $A R_{0.5}$ & $0.490(0.326)$ & $0.368(0.292)$ & $0.431(0.316)$ & $0.388(0.291)$ \\
\hline & $A R_{-0.5}$ & $0.978(0.256)$ & $0.996(0.301)$ & $0.928(0.224)$ & $0.997(0.301)$ \\
\hline & $M A_{0.2}$ & $0.775(0.335)$ & $0.780(0.334)$ & $0.653(0.307)$ & $0.790(0.335)$ \\
\hline
\end{tabular}

Table 2.29: Simultaneous coverage probabilities (average median interval length) over $G$ for $k=4$ and $a=0.3$. 
2.C Additional simulation Results

\begin{tabular}{|c|c|c|c|c|c|}
\hline \multirow[b]{2}{*}{$h$} & \multirow[b]{2}{*}{ DGP } & \multirow{2}{*}{$\frac{-}{\text { SWB }}$} & \multirow{2}{*}{$\begin{array}{c}\gamma=0 \\
\mathrm{WB}\end{array}$} & \multicolumn{2}{|c|}{$\gamma=0.2$} \\
\hline & & & & AWB & DWB \\
\hline \multirow{5}{*}{0.02} & 0 & $0.926(0.712)$ & $0.946(0.725)$ & $0.952(0.708)$ & $0.945(0.724)$ \\
\hline & $A R_{0.2}$ & $0.861(0.682)$ & $0.902(0.704)$ & $0.919(0.706)$ & $0.903(0.705)$ \\
\hline & $A R_{0.5}$ & $0.673(0.531)$ & $0.781(0.599)$ & $0.820(0.622)$ & $0.780(0.596)$ \\
\hline & $A R_{-0.5}$ & $0.960(0.547)$ & $0.989(0.618)$ & $0.988(0.565)$ & $0.989(0.614)$ \\
\hline & $M A_{0.2}$ & $0.872(0.682)$ & $0.910(0.706)$ & $0.924(0.706)$ & $0.911(0.705)$ \\
\hline \multirow{5}{*}{0.04} & 0 & $0.923(0.542)$ & $0.951(0.561)$ & $0.951(0.555)$ & $0.951(0.561)$ \\
\hline & $A R_{0.2}$ & $0.861(0.518)$ & $0.901(0.546)$ & $0.913(0.556)$ & $0.902(0.548)$ \\
\hline & $A R_{0.5}$ & $0.668(0.405)$ & $0.769(0.474)$ & $0.808(0.506)$ & $0.773(0.473)$ \\
\hline & $A R_{-0.5}$ & $0.971(0.419)$ & $0.996(0.487)$ & $0.993(0.449)$ & $0.995(0.484)$ \\
\hline & $M A_{0.2}$ & $0.874(0.520)$ & $0.913(0.549)$ & $0.921(0.557)$ & $0.912(0.548)$ \\
\hline \multirow{5}{*}{0.06} & 0 & $0.926(0.459)$ & $0.957(0.487)$ & $0.955(0.487)$ & $0.956(0.487)$ \\
\hline & $A R_{0.2}$ & $0.864(0.439)$ & $0.909(0.475)$ & $0.920(0.489)$ & $0.910(0.476)$ \\
\hline & $A R_{0.5}$ & $0.668(0.344)$ & $0.781(0.417)$ & $0.819(0.450)$ & $0.784(0.417)$ \\
\hline & $A R_{-0.5}$ & $0.980(0.356)$ & $0.997(0.428)$ & $0.995(0.402)$ & $0.997(0.425)$ \\
\hline & $M A_{0.2}$ & $0.877(0.440)$ & $0.920(0.476)$ & $0.927(0.489)$ & $0.919(0.476)$ \\
\hline \multirow[b]{2}{*}{$h$} & & \multicolumn{2}{|c|}{$\gamma=0.4$} & \multicolumn{2}{|c|}{$\gamma=0.6$} \\
\hline & DGP & AWB & DWB & AWB & DWB \\
\hline \multirow{5}{*}{0.02} & 0 & $0.942(0.648)$ & $0.945(0.724)$ & $0.904(0.549)$ & $0.947(0.726)$ \\
\hline & $A R_{0.2}$ & $0.913(0.660)$ & $0.903(0.701)$ & $0.868(0.576)$ & $0.901(0.702)$ \\
\hline & $A R_{0.5}$ & $0.829(0.610)$ & $0.781(0.594)$ & $0.791(0.557)$ & $0.779(0.596)$ \\
\hline & $A R_{-0.5}$ & $0.982(0.492)$ & $0.989(0.616)$ & $0.958(0.392)$ & $0.989(0.616)$ \\
\hline & $M A_{0.2}$ & $0.918(0.660)$ & $0.908(0.703)$ & $0.879(0.574)$ & $0.912(0.706)$ \\
\hline \multirow{5}{*}{0.04} & 0 & $0.940(0.519)$ & $0.949(0.561)$ & $0.905(0.454)$ & $0.951(0.562)$ \\
\hline & $A R_{0.2}$ & $0.905(0.534)$ & $0.901(0.543)$ & $0.869(0.479)$ & $0.899(0.544)$ \\
\hline & $A R_{0.5}$ & $0.818(0.510)$ & $0.769(0.471)$ & $0.795(0.484)$ & $0.767(0.472)$ \\
\hline & $A R_{-0.5}$ & $0.989(0.400)$ & $0.995(0.486)$ & $0.970(0.333)$ & $0.995(0.486)$ \\
\hline & $M A_{0.2}$ & $0.912(0.534)$ & $0.910(0.546)$ & $0.880(0.478)$ & $0.912(0.548)$ \\
\hline \multirow{5}{*}{0.06} & 0 & $0.945(0.464)$ & $0.953(0.486)$ & $0.916(0.417)$ & $0.956(0.4$ \\
\hline & Al & $0.913(0.477)$ & $0.906(0.473)$ & $0.884(0.441)$ & $0.906(0.474)$ \\
\hline & $A R_{0.5}$ & $0.829(0.462)$ & $0.779(0.415)$ & $0.816(0.448)$ & $0.779(0.415)$ \\
\hline & $A R_{-0.5}$ & $0.992(0.368)$ & $0.997(0.427)$ & $0.978(0.321)$ & $0.997(0.428)$ \\
\hline & $M A_{0.2}$ & $0.919(0.478)$ & $0.915(0.475)$ & $0.893(0.439)$ & $0.918(0.475)$ \\
\hline
\end{tabular}

Table 2.30: Pointwise coverage probabilities (average median interval length) for $k=4$ and $a=0.5$. 


\begin{tabular}{|c|c|c|c|c|c|}
\hline \multirow[b]{2}{*}{$h$} & \multirow[b]{2}{*}{ DGP } & \multirow{2}{*}{$\frac{-}{\mathrm{SWB}}$} & \multirow{2}{*}{$\begin{array}{c}\gamma=0 \\
\mathrm{WB}\end{array}$} & \multicolumn{2}{|c|}{$\gamma=0.2$} \\
\hline & & & & AWB & DWB \\
\hline \multirow{5}{*}{0.02} & 0 & $0.883(0.657)$ & $0.926(0.669)$ & $0.933(0.653)$ & $0.921(0.668)$ \\
\hline & $A R_{0.2}$ & $0.784(0.630)$ & $0.865(0.650)$ & $0.898(0.651)$ & $0.871(0.651)$ \\
\hline & $A R_{0.5}$ & $0.384(0.490)$ & $0.665(0.552)$ & $0.739(0.574)$ & $0.665(0.551)$ \\
\hline & $A R_{-0.5}$ & $0.988(0.505)$ & $0.984(0.570)$ & $0.979(0.522)$ & $0.980(0.567)$ \\
\hline & $M A_{0.2}$ & $0.782(0.630)$ & $0.863(0.651)$ & $0.894(0.650)$ & $0.866(0.650)$ \\
\hline \multirow{5}{*}{0.04} & 0 & $0.879(0.502)$ & $0.919(0.520)$ & $0.918(0.514)$ & $0.912(0.519)$ \\
\hline & $A R_{0.2}$ & $0.739(0.478)$ & $0.825(0.505)$ & $0.867(0.514)$ & $0.842(0.506)$ \\
\hline & $A R_{0.5}$ & $0.317(0.374)$ & $0.558(0.438)$ & $0.629(0.468)$ & $0.559(0.438)$ \\
\hline & $A R_{-0.5}$ & $0.987(0.388)$ & $0.991(0.450)$ & $0.985(0.416)$ & $0.986(0.448)$ \\
\hline & $M A_{0.2}$ & $0.766(0.481)$ & $0.839(0.507)$ & $0.864(0.516)$ & $0.833(0.507)$ \\
\hline \multirow{5}{*}{0.06} & 0 & $0.881(0.425)$ & $0.921(0.451)$ & $0.925(0.451)$ & $0.923(0.451)$ \\
\hline & $A R_{0.2}$ & $0.749(0.406)$ & $0.821(0.439)$ & $0.841(0.452)$ & $0.832(0.440)$ \\
\hline & $A R_{0.5}$ & $0.331(0.318)$ & $0.539(0.387)$ & $0.610(0.417)$ & $0.532(0.385)$ \\
\hline & $A R_{-0.5}$ & $0.996(0.329)$ & $0.998(0.396)$ & $0.991(0.373)$ & $0.992(0.393)$ \\
\hline & $M A_{0.2}$ & $0.782(0.408)$ & $0.845(0.440)$ & $0.867(0.453)$ & $0.856(0.440)$ \\
\hline \multirow[b]{2}{*}{$h$} & \multirow[b]{2}{*}{ DGP } & \multicolumn{2}{|c|}{$\gamma=0.4$} & \multicolumn{2}{|c|}{$\gamma=0.6$} \\
\hline & & AWB & DWB & AWB & DWB \\
\hline \multirow{5}{*}{0.02} & 0 & $0.934(0.597)$ & $0.929(0.668)$ & $0.864(0.507)$ & $0.936(0.669)$ \\
\hline & $A R_{0.2}$ & $0.881(0.609)$ & $0.861(0.646)$ & $0.813(0.530)$ & $0.868(0.648)$ \\
\hline & $A R_{0.5}$ & $0.745(0.562)$ & $0.660(0.548)$ & $0.665(0.513)$ & $0.659(0.549)$ \\
\hline & $A R_{-0.5}$ & $0.963(0.454)$ & $0.979(0.569)$ & $0.912(0.362)$ & $0.979(0.568)$ \\
\hline & $M A_{0.2}$ & $0.897(0.609)$ & $0.884(0.649)$ & $0.810(0.529)$ & $0.888(0.650)$ \\
\hline \multirow{5}{*}{0.04} & 0 & $0.899(0.480)$ & $0.928(0.519)$ & $0.786(0.419)$ & $0.919(0.520)$ \\
\hline & $A R_{0.2}$ & $0.833(0.494)$ & $0.821(0.503)$ & $0.766(0.443)$ & $0.837(0.504)$ \\
\hline & $A R_{0.5}$ & $0.640(0.471)$ & $0.548(0.435)$ & $0.585(0.447)$ & $0.562(0.436)$ \\
\hline & $A R_{-0.5}$ & $0.967(0.370)$ & $0.987(0.449)$ & $0.921(0.308)$ & $0.990(0.450)$ \\
\hline & $M A_{0.2}$ & $0.854(0.494)$ & $0.867(0.505)$ & $0.745(0.442)$ & $0.849(0.507)$ \\
\hline \multirow{5}{*}{0.06} & 0 & $0.905(0.430)$ & $0.931(0.450)$ & $0.796(0.385)$ & $0.923(0.450)$ \\
\hline & $A R_{0.2}$ & $0.814(0.442)$ & $0.825(0.437)$ & $0.742(0.408)$ & $0.836(0.439)$ \\
\hline & $A R_{0.5}$ & $0.647(0.427)$ & $0.534(0.383)$ & $0.610(0.414)$ & $0.540(0.384)$ \\
\hline & $A R_{-0.5}$ & $0.978(0.341)$ & $0.995(0.395)$ & $0.933(0.296)$ & $0.998(0.396)$ \\
\hline & $M A_{0.2}$ & $0.850(0.442)$ & $0.855(0.439)$ & $0.752(0.405)$ & $0.841(0.440)$ \\
\hline
\end{tabular}

Table 2.31: Simultaneous coverage probabilities (average median interval length) over $G_{s u b}$ for $k=4$ and $a=0.5$. 
2.C Additional simulation Results

\begin{tabular}{|c|c|c|c|c|c|}
\hline \multirow[b]{2}{*}{$h$} & \multirow[b]{2}{*}{ DGP } & \multirow{2}{*}{$\frac{-}{\text { SWB }}$} & \multirow{2}{*}{$\begin{array}{c}\gamma=0 \\
\mathrm{WB}\end{array}$} & \multicolumn{2}{|c|}{$\gamma=0.2$} \\
\hline & & & & AWB & DWB \\
\hline \multirow{5}{*}{0.02} & 0 & $0.848(0.520)$ & $0.904(0.530)$ & $0.918(0.517)$ & $0.905(0.529)$ \\
\hline & $A R_{02}$ & $0.685(0.499)$ & $0.822(0.514)$ & $0.876(0.515)$ & $0.849(0.515)$ \\
\hline & $A R_{0.5}$ & $0.229(0.388)$ & $0.576(0.437)$ & $0.655(0.455)$ & $0.560(0.436)$ \\
\hline & $A R_{-0.5}$ & $0.984(0.399)$ & $0.979(0.451)$ & $0.974(0.413)$ & $0.977(0.449)$ \\
\hline & $M A_{0.2}$ & $0.714(0.499)$ & $0.837(0.516)$ & $0.873(0.515)$ & $0.843(0.515)$ \\
\hline \multirow{5}{*}{0.04} & 0 & $0.854(0.390)$ & $0.900(0.404)$ & $0.905(0.399)$ & $0.906(0.403)$ \\
\hline & $A R_{0.2}$ & $0.668(0.372)$ & $0.770(0.392)$ & $0.822(0.400)$ & $0.793(0.393)$ \\
\hline & $A R_{0.5}$ & $0.153(0.291)$ & $0.410(0.340)$ & $0.498(0.364)$ & $0.398(0.340)$ \\
\hline & $A R_{-0.5}$ & $0.985(0.301)$ & $0.991(0.350)$ & $0.983(0.323)$ & $0.986(0.347)$ \\
\hline & $M A_{0.2}$ & $0.687(0.373)$ & $0.809(0.394)$ & $0.825(0.400)$ & $0.794(0.394)$ \\
\hline \multirow{5}{*}{0.06} & 0 & $0.860(0.327)$ & $0.898(0$ & $0.912(0.347)$ & 0.914 \\
\hline & $A R_{0.2}$ & $0.671(0.313)$ & $0.757(0.339)$ & $0.804(0.349)$ & $0.785(0.339)$ \\
\hline & $A R_{0.5}$ & $0.151(0.245)$ & $0.376(0.298)$ & $0.425(0.321)$ & $0.360(0.297)$ \\
\hline & $A R_{-0.5}$ & $0.996(0.254)$ & $0.996(0.305)$ & $0.992(0.287)$ & $0.994(0.303)$ \\
\hline & $M A_{0.2}$ & $0.719(0.314)$ & $0.804(0.339)$ & $0.820(0.349)$ & $0.801(0.340)$ \\
\hline \multirow[b]{2}{*}{$h$} & & \multicolumn{2}{|c|}{$\gamma=0.4$} & \multicolumn{2}{|c|}{$\gamma=0.6$} \\
\hline & DGP & AWB & DWB & AWB & DWB \\
\hline \multirow{5}{*}{0.02} & 0 & $0.910(0.473)$ & $0.912(0.529)$ & $0.837(0.401)$ & $0.928(0.530)$ \\
\hline & $A R_{0.2}$ & $0.866(0.483)$ & $0.823(0.512)$ & $0.731(0.421)$ & $0.815(0.513)$ \\
\hline & $A R_{0.5}$ & $0.629(0.447)$ & $0.558(0.434)$ & $0.554(0.408)$ & $0.564(0.435)$ \\
\hline & $A R_{-0.5}$ & $0.956(0.359)$ & $0.973(0.450)$ & $0.898(0.286)$ & $0.976(0.450)$ \\
\hline & $M A_{0.2}$ & $0.858(0.483)$ & $0.846(0.513)$ & $0.769(0.419)$ & $0.861(0.515)$ \\
\hline \multirow{5}{*}{0.04} & 0 & $0.856(0.373)$ & $0.904(0$ & $0.734(0.326)$ & $0.906(0.404)$ \\
\hline & $A R_{0.2}$ & $0.770(0.384)$ & $0.768(0.390)$ & $0.646(0.344)$ & $0.756(0.391)$ \\
\hline & $A R_{0.5}$ & $0.513(0.367)$ & $0.401(0.338)$ & $0.458(0.348)$ & $0.417(0.339)$ \\
\hline & $A R_{-0.5}$ & $0.961(0.287)$ & $0.989(0.348)$ & $0.900(0.239)$ & $0.994(0.349)$ \\
\hline & $M A_{0.2}$ & $0.790(0.384)$ & $0.805(0.392)$ & $0.662(0.344)$ & $0.795(0.393)$ \\
\hline \multirow{5}{*}{0.06} & 0 & $0.852(0.331)$ & $0.902(0.347)$ & $0.735(0.297)$ & $0.900(0.347)$ \\
\hline & $A R_{0.2}$ & $0.747(0.341)$ & $0.752(0.337)$ & $0.621(0.315)$ & $0.774(0.338)$ \\
\hline & $A R_{0.5}$ & $0.495(0.330)$ & $0.365(0.295)$ & $0.447(0.320)$ & $0.394(0.296)$ \\
\hline & $A R_{-}$ & $0.978(0.262)$ & $0.997(0.304)$ & $0.932(0.228)$ & $0.997(0.305)$ \\
\hline & $M A_{0.2}$ & $0.782(0.341)$ & $0.780(0.338)$ & $0.661(0.313)$ & $0.788(0.339)$ \\
\hline
\end{tabular}

Table 2.32: Simultaneous coverage probabilities (average median interval length) over $G$ for $k=4$ and $a=0.5$. 


\begin{tabular}{|c|c|c|c|c|c|}
\hline \multirow[b]{2}{*}{$h$} & \multirow[b]{2}{*}{ DGP } & \multirow{2}{*}{$\frac{-}{\mathrm{SWB}}$} & \multirow{2}{*}{$\begin{array}{c}\gamma=0 \\
\mathrm{WB}\end{array}$} & \multicolumn{2}{|c|}{$\gamma=0.2$} \\
\hline & & & & AWB & DWB \\
\hline \multirow{5}{*}{0.02} & 0 & $0.923(0.701)$ & $0.945(0.719)$ & $0.951(0.702)$ & $0.945(0.717)$ \\
\hline & $A R_{0.2}$ & $0.860(0.672)$ & $0.902(0.697)$ & $0.918(0.700)$ & $0.903(0.698)$ \\
\hline & $A R_{0.5}$ & $0.674(0.521)$ & $0.783(0.594)$ & $0.820(0.617)$ & $0.781(0.593)$ \\
\hline & $A R_{-0.5}$ & $0.959(0.536)$ & $0.989(0.611)$ & $0.988(0.560)$ & $0.989(0.607)$ \\
\hline & $M A_{0.2}$ & $0.870(0.671)$ & $0.910(0.700)$ & $0.924(0.700)$ & $0.910(0.698)$ \\
\hline \multirow{5}{*}{0.04} & 0 & $0.922(0.537)$ & $0.952(0.564)$ & $0.952(0.559)$ & $0.952(0.564)$ \\
\hline & $A R_{0.2}$ & $0.860(0.513)$ & $0.903(0.548)$ & $0.914(0.559)$ & $0.903(0.550)$ \\
\hline & $A R_{0.5}$ & $0.668(0.401)$ & $0.772(0.477)$ & $0.811(0.509)$ & $0.776(0.477)$ \\
\hline & $A R_{-0.5}$ & $0.970(0.415)$ & $0.996(0.489)$ & $0.993(0.453)$ & $0.995(0.486)$ \\
\hline & $M A_{0.2}$ & $0.873(0.515)$ & $0.914(0.552)$ & $0.922(0.561)$ & $0.913(0.551)$ \\
\hline \multirow{5}{*}{0.06} & 0 & $0.925(0.458)$ & $0.958(0.494)$ & $0.957(0.494)$ & $0.956(0.493)$ \\
\hline & $A R_{0.2}$ & $0.863(0.438)$ & $0.911(0.481)$ & $0.922(0.496)$ & $0.911(0.482)$ \\
\hline & $A R_{0.5}$ & $0.666(0.343)$ & $0.784(0.424)$ & $0.823(0.458)$ & $0.787(0.424)$ \\
\hline & $A R_{-0.5}$ & $0.978(0.356)$ & $0.997(0.433)$ & $0.995(0.408)$ & $0.997(0.430)$ \\
\hline & $M A_{0.2}$ & $0.876(0.440)$ & $0.922(0.483)$ & $0.928(0.497)$ & $0.920(0.482)$ \\
\hline & \multirow[b]{2}{*}{ DGP } & \multicolumn{2}{|c|}{$\gamma=0.4$} & \multicolumn{2}{|c|}{$\gamma=0.6$} \\
\hline & & AWB & DWB & AWB & DWB \\
\hline \multirow{5}{*}{0.02} & 0 & $0.941(0.643)$ & $0.945(0.717)$ & $0.903(0.546)$ & $0.947(0.719)$ \\
\hline & $A R_{0.2}$ & $0.912(0.655)$ & $0.903(0.694)$ & $0.867(0.570)$ & $0.901(0.695)$ \\
\hline & $A R_{0.5}$ & $0.829(0.605)$ & $0.783(0.590)$ & $0.792(0.550)$ & $0.782(0.591)$ \\
\hline & $A R_{-0.5}$ & $0.982(0.490)$ & $0.989(0.610)$ & $0.958(0.392)$ & $0.989(0.610)$ \\
\hline & $M A_{0.2}$ & $0.916(0.654)$ & $0.909(0.697)$ & $0.878(0.569)$ & $0.912(0.700)$ \\
\hline \multirow{5}{*}{0.04} & 0 & $0.941(0.525)$ & $0.950(0.563)$ & $0.907(0.460)$ & $0.952(0.563)$ \\
\hline & $A R_{0.2}$ & $0.907(0.538)$ & $0.903(0.546)$ & $0.872(0.484)$ & $0.901(0.547)$ \\
\hline & $A R_{0.5}$ & $0.820(0.513)$ & $0.772(0.474)$ & $0.799(0.487)$ & $0.771(0.475)$ \\
\hline & $A R_{-0.5}$ & $0.989(0.405)$ & $0.995(0.487)$ & $0.971(0.341)$ & $0.996(0.489)$ \\
\hline & $M A_{0.2}$ & $0.914(0.539)$ & $0.911(0.549)$ & $0.882(0.484)$ & $0.914(0.550)$ \\
\hline \multirow{5}{*}{0.06} & 0 & $0.947(0.474)$ & $0.954(0.492)$ & $0.919(0.427)$ & $0.956(0.493)$ \\
\hline & $A R_{0.2}$ & $0.916(0.486)$ & $0.908(0.479)$ & $0.887(0.451)$ & $0.909(0.481)$ \\
\hline & $A R_{0.5}$ & $0.833(0.470)$ & $0.783(0.421)$ & $0.820(0.456)$ & $0.782(0.422)$ \\
\hline & $A R_{-0.5}$ & $0.992(0.376)$ & $0.997(0.431)$ & $0.978(0.329)$ & $0.997(0.433)$ \\
\hline & $M A_{0.2}$ & $0.922(0.487)$ & $0.917(0.481)$ & $0.896(0.448)$ & $0.920(0.482)$ \\
\hline
\end{tabular}

Table 2.33: Pointwise coverage probabilities (average median interval length) for $k=4$ and $a=0.7$. 
2.C Additional simulation Results

\begin{tabular}{|c|c|c|c|c|c|}
\hline \multirow[b]{2}{*}{$h$} & \multirow[b]{2}{*}{ DGP } & \multirow{2}{*}{$\frac{-}{\text { SWB }}$} & \multirow{2}{*}{$\begin{array}{c}\gamma=0 \\
\mathrm{WB}\end{array}$} & \multicolumn{2}{|c|}{$\gamma=0.2$} \\
\hline & & & & AWB & DWB \\
\hline \multirow{5}{*}{0.02} & 0 & $0.884(0.646)$ & $0.929(0.662)$ & $0.932(0.647)$ & $0.924(0.661)$ \\
\hline & $A R_{0.2}$ & $0.772(0.619)$ & $0.866(0.643)$ & $0.905(0.645)$ & $0.876(0.645)$ \\
\hline & $A R_{0.5}$ & $0.391(0.481)$ & $0.665(0.547)$ & $0.746(0.569)$ & $0.667(0.547)$ \\
\hline & $A R_{-0.5}$ & $0.990(0.495)$ & $0.984(0.563)$ & $0.980(0.518)$ & $0.981(0.560)$ \\
\hline & $M A_{0.2}$ & $0.776(0.619)$ & $0.872(0.645)$ & $0.889(0.645)$ & $0.870(0.644)$ \\
\hline \multirow{5}{*}{0.04} & 0 & $0.872(0.496)$ & $0.924(0.521)$ & $0.918(0.517)$ & $0.915(0.521)$ \\
\hline & $A R_{0.2}$ & $0.745(0.474)$ & $0.834(0.507)$ & $0.870(0.517)$ & $0.850(0.508)$ \\
\hline & $A R_{0.5}$ & $0.314(0.370)$ & $0.553(0.441)$ & $0.634(0.471)$ & $0.565(0.440)$ \\
\hline & $A R_{-0.5}$ & $0.986(0.384)$ & $0.990(0.451)$ & $0.983(0.419)$ & $0.987(0.449)$ \\
\hline & $M A_{0.2}$ & $0.764(0.476)$ & $0.844(0.509)$ & $0.859(0.519)$ & $0.830(0.509)$ \\
\hline \multirow{5}{*}{0.06} & 0 & $0.884(0.424)$ & $0.923(0.456)$ & $0.930(0.457)$ & 0.930 \\
\hline & $A R_{0.2}$ & $0.743(0.405)$ & $0.830(0.444)$ & $0.848(0.459)$ & $0.840(0.445)$ \\
\hline & $A R_{0.5}$ & $0.327(0.317)$ & $0.547(0.392)$ & $0.615(0.424)$ & $0.539(0.391)$ \\
\hline & $A R_{-0.5}$ & $0.997(0.329)$ & $0.998(0.400)$ & $0.990(0.378)$ & $0.990(0.397)$ \\
\hline & $M A_{0.2}$ & $0.787(0.406)$ & $0.855(0.446)$ & $0.872(0.459)$ & $0.861(0.446)$ \\
\hline \multirow[b]{2}{*}{$h$} & & \multicolumn{2}{|c|}{$\gamma=0.4$} & \multicolumn{2}{|c|}{$\gamma=0.6$} \\
\hline & DGP & AWB & DWB & AWB & DWB \\
\hline \multirow{5}{*}{0.02} & 0 & $0.938(0.594)$ & $0.934(0.661)$ & $0.877(0.503)$ & $0.939(0.663)$ \\
\hline & $A R_{0.2}$ & $0.887(0.604)$ & $0.863(0.640)$ & $0.816(0.525)$ & $0.870(0.641)$ \\
\hline & $A R_{0.5}$ & $0.751(0.558)$ & $0.661(0.545)$ & $0.665(0.507)$ & $0.663(0.545)$ \\
\hline & $A R_{-0.5}$ & $0.965(0.453)$ & $0.980(0.562)$ & $0.915(0.362)$ & $0.980(0.562)$ \\
\hline & $M A_{0.2}$ & $0.899(0.604)$ & $0.888(0.643)$ & $0.826(0.525)$ & $0.892(0.645)$ \\
\hline \multirow{5}{*}{0.04} & 0 & $0.903(0.485)$ & $0.925(0.5$ & $0.795(0.425)$ & $0.916(0.520)$ \\
\hline & $A R_{0.2}$ & $0.840(0.498)$ & $0.820(0.505)$ & $0.778(0.447)$ & $0.835(0.505)$ \\
\hline & $A R_{0.5}$ & $0.651(0.474)$ & $0.562(0.438)$ & $0.594(0.449)$ & $0.564(0.439)$ \\
\hline & $A R_{-0.5}$ & $0.970(0.375)$ & $0.987(0.449)$ & $0.925(0.315)$ & $0.991(0.452)$ \\
\hline & $M A_{0.2}$ & $0.867(0.498)$ & $0.871(0.507)$ & $0.750(0.447)$ & $0.854(0.508)$ \\
\hline \multirow{5}{*}{0.06} & 0 & $0.913(0.438)$ & $0.932(0.455)$ & 0.812( & $0.926(0.455)$ \\
\hline & $A R_{0.2}$ & $0.820(0.450)$ & $0.840(0.442)$ & $0.761(0.416)$ & $0.838(0.444)$ \\
\hline & $A R_{0.5}$ & $0.654(0.434)$ & $0.545(0.389)$ & $0.612(0.421)$ & $0.544(0.390)$ \\
\hline & $A R_{-}$ & $0.981(0.348)$ & $0.995(0.398)$ & $0.936(0.304)$ & $0.998(0.400)$ \\
\hline & $M A_{0.2}$ & $0.863(0.450)$ & $0.868(0.444)$ & $0.759(0.413)$ & $0.859(0.446)$ \\
\hline
\end{tabular}

Table 2.34: Simultaneous coverage probabilities (average median interval length) over $G_{\text {sub }}$ for $k=4$ and $a=0.7$. 


\begin{tabular}{|c|c|c|c|c|c|}
\hline \multirow[b]{2}{*}{$h$} & \multirow[b]{2}{*}{ DGP } & \multirow{2}{*}{$\frac{-}{\text { SWB }}$} & \multirow{2}{*}{$\begin{array}{c}\gamma=0 \\
\mathrm{WB}\end{array}$} & \multicolumn{2}{|c|}{$\gamma=0.2$} \\
\hline & & & & AWB & DWB \\
\hline \multirow{5}{*}{0.02} & 0 & $0.846(0.512)$ & $0.902(0.525)$ & $0.912(0.512)$ & $0.903(0.524)$ \\
\hline & $A R_{0.2}$ & $0.693(0.490)$ & $0.812(0.509)$ & $0.870(0.511)$ & $0.836(0.510)$ \\
\hline & $A R_{0.5}$ & $0.233(0.381)$ & $0.566(0.434)$ & $0.648(0.451)$ & $0.551(0.433)$ \\
\hline & $A R_{-0.5}$ & $0.986(0.392)$ & $0.980(0.446)$ & $0.974(0.409)$ & $0.977(0.444)$ \\
\hline & $M A_{0.2}$ & $0.712(0.490)$ & $0.828(0.511)$ & $0.871(0.511)$ & $0.836(0.510)$ \\
\hline \multirow{5}{*}{0.04} & 0 & $0.850(0.385)$ & $0.904(0.405)$ & $0.909(0.401)$ & $0.910(0.405)$ \\
\hline & $A R_{0.2}$ & $0.675(0.368)$ & $0.769(0.393)$ & $0.821(0.402)$ & $0.796(0.395)$ \\
\hline & $A R_{0.5}$ & $0.155(0.288)$ & $0.417(0.342)$ & $0.512(0.366)$ & $0.400(0.341)$ \\
\hline & $A R_{-0.5}$ & $0.987(0.297)$ & $0.990(0.350)$ & $0.987(0.325)$ & $0.987(0.349)$ \\
\hline & $M A_{0.2}$ & $0.691(0.370)$ & $0.812(0.396)$ & $0.827(0.403)$ & $0.798(0.395)$ \\
\hline \multirow{5}{*}{0.06} & 0 & $0.857(0.326)$ & $0.906(0.351)$ & $0.915(0.352)$ & $0.922(0.351)$ \\
\hline & $A R_{0.2}$ & $0.674(0.312)$ & $0.763(0.343)$ & $0.811(0.353)$ & $0.793(0.343)$ \\
\hline & $A R_{0.5}$ & $0.146(0.244)$ & $0.392(0.302)$ & $0.447(0.326)$ & $0.364(0.301)$ \\
\hline & $A R_{-0.5}$ & $0.997(0.253)$ & $0.995(0.308)$ & $0.991(0.290)$ & $0.996(0.306)$ \\
\hline & $M A_{0.2}$ & $0.719(0.313)$ & $0.815(0.344)$ & $0.832(0.354)$ & $0.806(0.343)$ \\
\hline \multirow[b]{2}{*}{$h$} & \multirow[b]{2}{*}{ DGP } & \multicolumn{2}{|c|}{$\gamma=0.4$} & \multicolumn{2}{|c|}{$\gamma=0.6$} \\
\hline & & AWB & DWB & AWB & DWB \\
\hline \multirow{5}{*}{0.02} & 0 & $0.904(0.470)$ & $0.913(0.524)$ & $0.812(0.399)$ & $0.924(0.525)$ \\
\hline & $A R_{0.2}$ & $0.854(0.479)$ & $0.827(0.507)$ & $0.712(0.417)$ & $0.813(0.508)$ \\
\hline & $A R_{0.5}$ & $0.610(0.443)$ & $0.554(0.431)$ & $0.538(0.404)$ & $0.574(0.431)$ \\
\hline & $A R_{-0.5}$ & $0.949(0.358)$ & $0.973(0.445)$ & $0.887(0.286)$ & $0.975(0.445)$ \\
\hline & $M A_{0.2}$ & $0.855(0.479)$ & $0.841(0.508)$ & $0.745(0.417)$ & $0.853(0.511)$ \\
\hline \multirow{5}{*}{0.04} & 0 & $0.865(0.376)$ & $0.907(0.404)$ & $0.726(0.330)$ & $0.915(0.404)$ \\
\hline & $A R_{0.2}$ & $0.776(0.387)$ & $0.780(0.392)$ & $0.649(0.348)$ & $0.765(0.392)$ \\
\hline & $A R_{0.5}$ & $0.520(0.368)$ & $0.400(0.340)$ & $0.465(0.350)$ & $0.417(0.341)$ \\
\hline & $A R_{-0.5}$ & $0.965(0.290)$ & $0.990(0.349)$ & $0.913(0.244)$ & $0.993(0.350)$ \\
\hline & $M A_{0.2}$ & $0.784(0.387)$ & $0.809(0.393)$ & $0.659(0.348)$ & $0.803(0.394)$ \\
\hline \multirow{5}{*}{0.06} & 0 & $0.854(0.337)$ & $0.904(0.351)$ & $0.746(0.304)$ & $0.906(0.351)$ \\
\hline & $A R_{0.2}$ & $0.758(0.347)$ & $0.767(0.341)$ & $0.640(0.321)$ & $0.779(0.342)$ \\
\hline & $A R_{0.5}$ & $0.511(0.335)$ & $0.379(0.300)$ & $0.462(0.325)$ & $0.415(0.300)$ \\
\hline & $A R_{-0.5}$ & $0.977(0.268)$ & $0.996(0.307)$ & $0.934(0.234)$ & $0.997(0.308)$ \\
\hline & $M A_{0.2}$ & $0.784(0.347)$ & $0.788(0.343)$ & $0.678(0.319)$ & $0.804(0.343)$ \\
\hline
\end{tabular}

Table 2.35: Simultaneous coverage probabilities (average median interval length) over $G$ for $k=4$ and $a=0.7$. 
2.C.3 Missing data - varying the bandwidth 


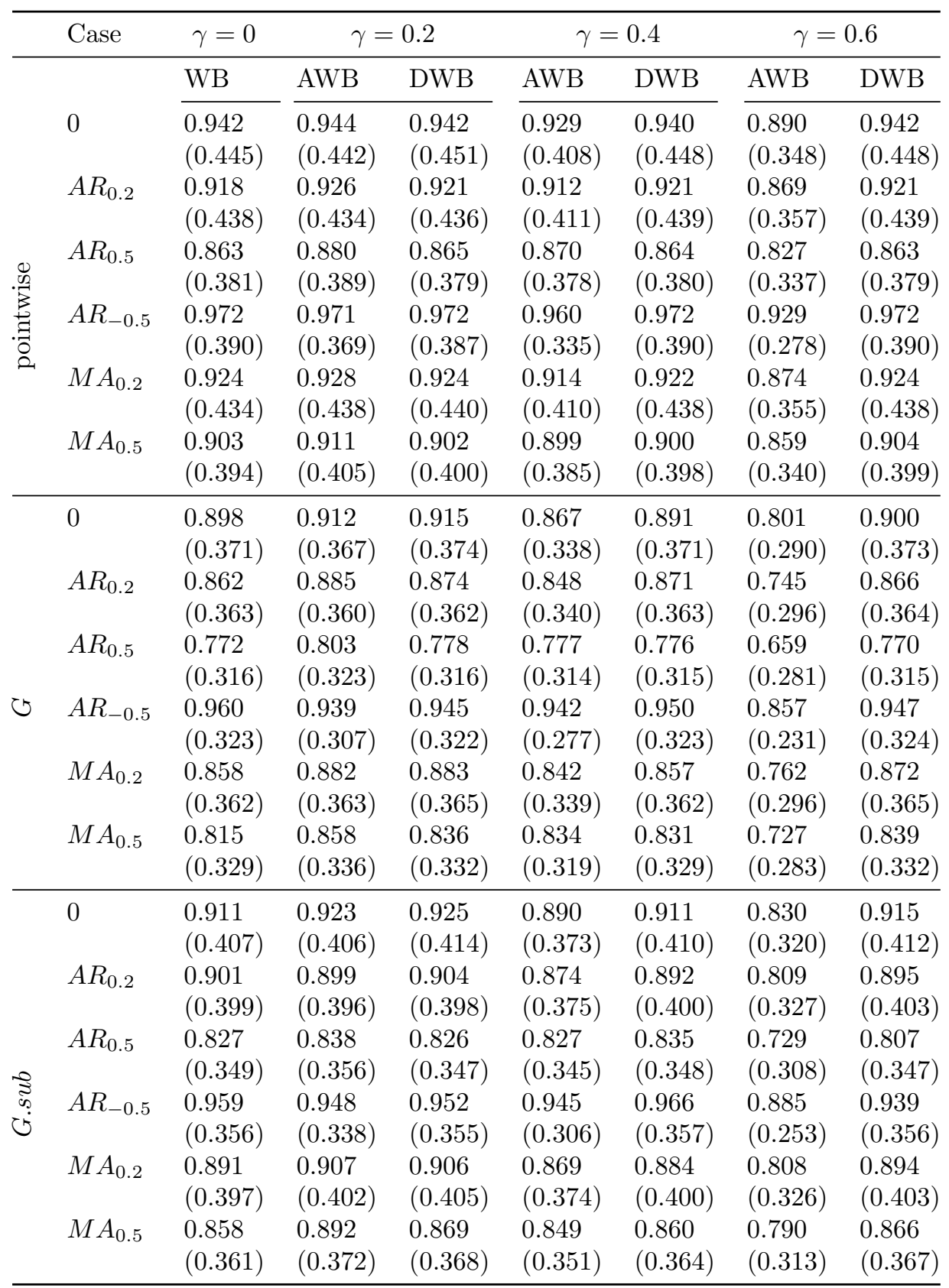

Table 2.36: Coverage with missing data $(h=0.02)$ 
2.C Additional simulation Results

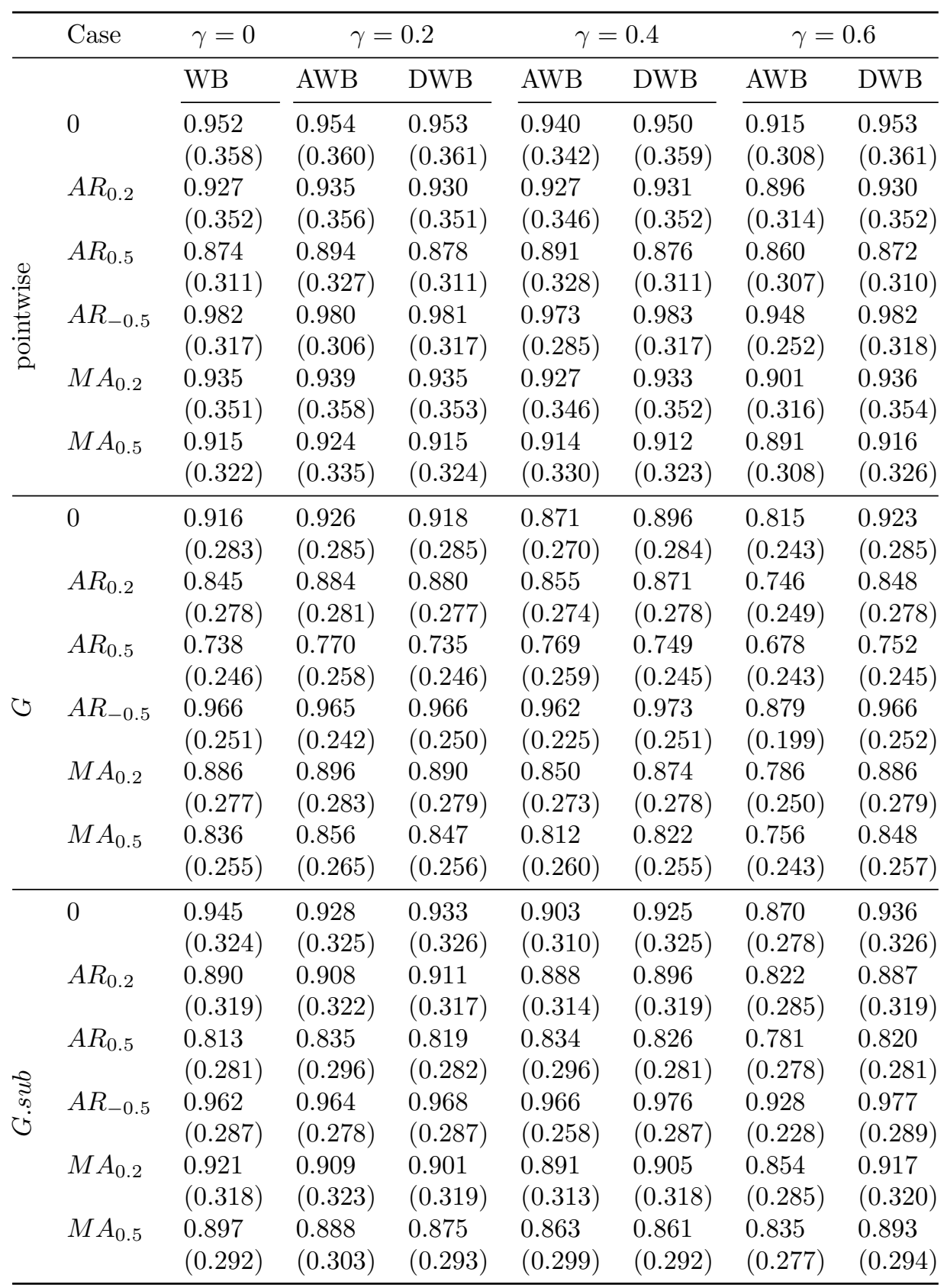

Table 2.37: Coverage with missing data $(h=0.04)$ 


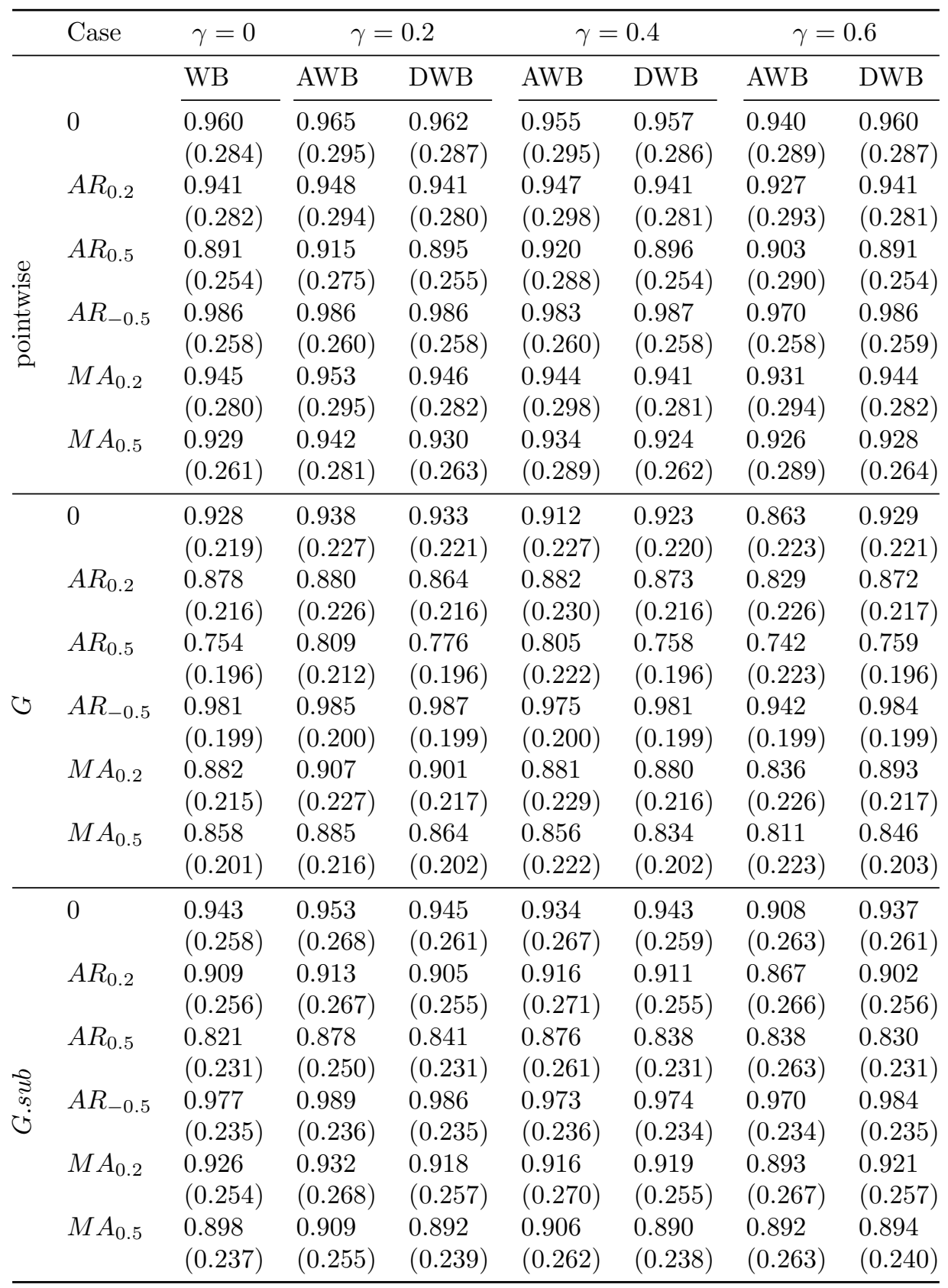

Table 2.38: Coverage with missing data $(h=0.08)$ 


\section{D Additional graphs for varying the number of Fourier terms}

In this section, we show additional plots of the seasonally adjusted ethane series together with the nonparametric trend plus confidence bands as in Figure 2.4 above. The difference here are the number of Fourier terms $(M)$ used to handle seasonality. In the chapter, the results shown are for $M=3$. Here, we present additional results for $M=1,2,4$, which we refer to in the core part of the chapter. They are plotted in Figures 2.6, 2.7 and 2.8, respectively. As pointed out there, we observe that the resulting trend curves and confidence bands are almost identical to Figure 4. Lastly, Figure 2.9 shows that when we increase the bandwidth, there is enough smoothing due to the nonparametric estimator such that no initial regression on Fourier terms is needed. Again, the shape is very similar to the previous graphs. 


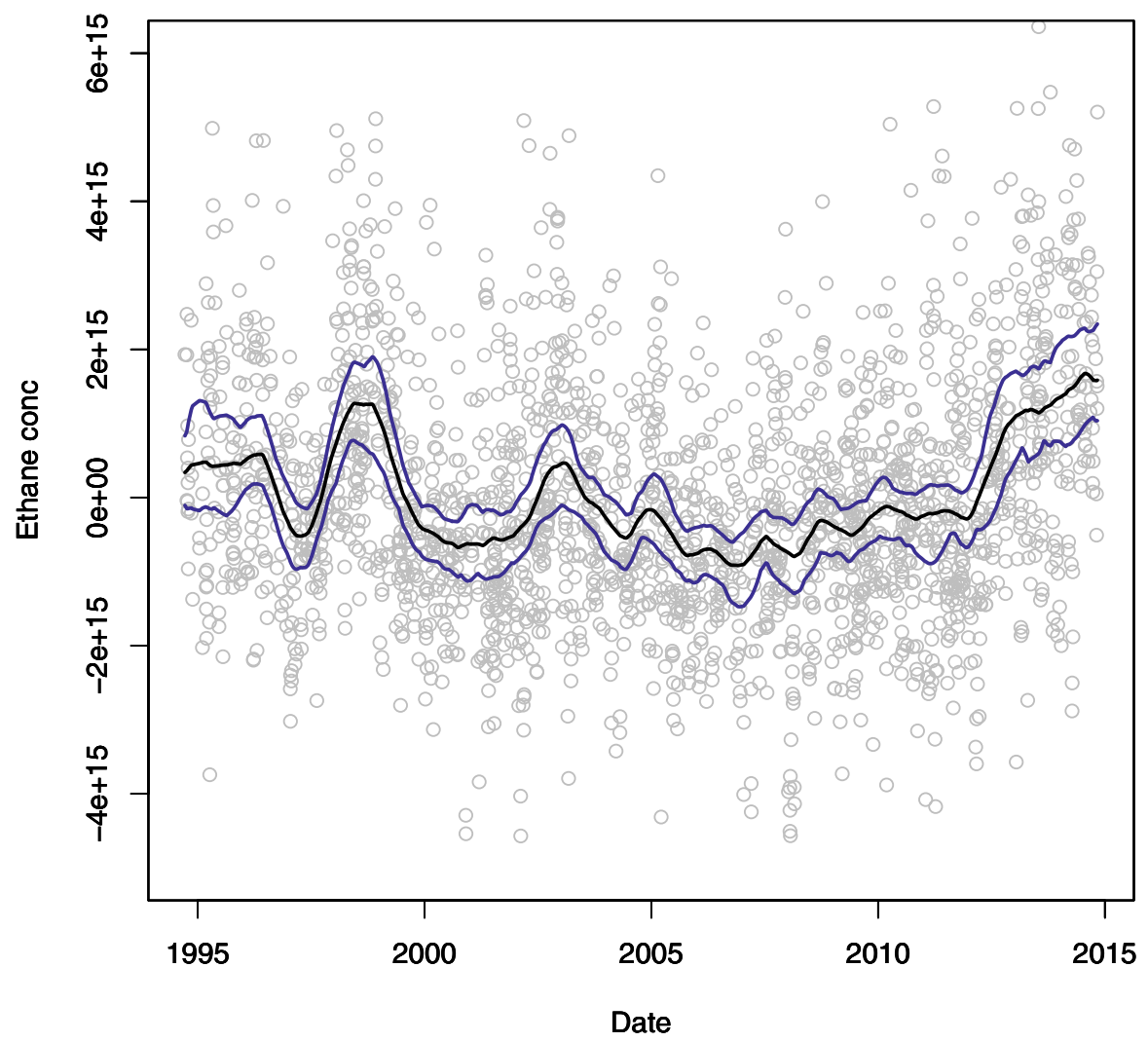

Figure 2.6: $M=1, h=0.03$ 


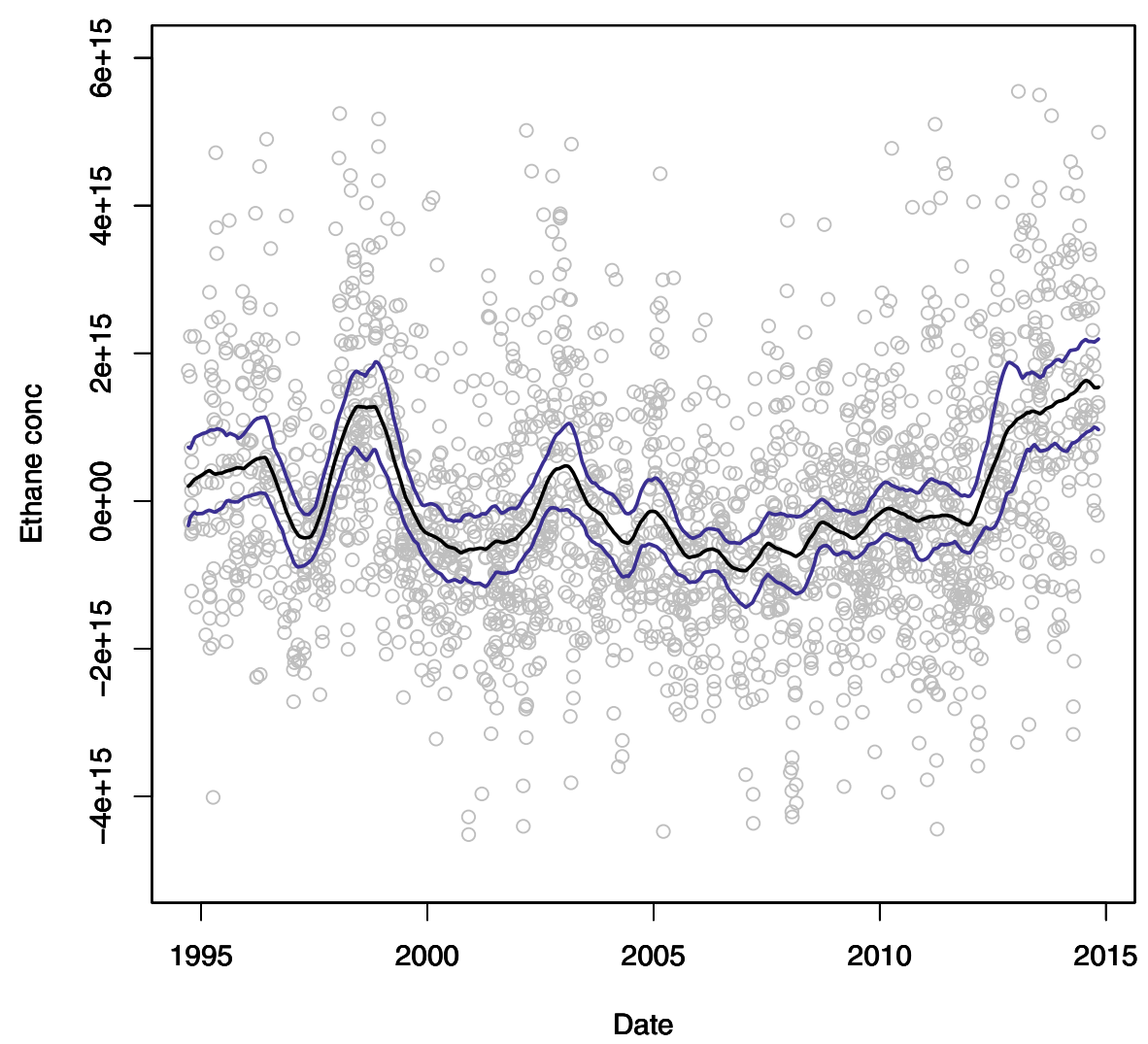

Figure 2.7: $M=2, h=0.03$ 


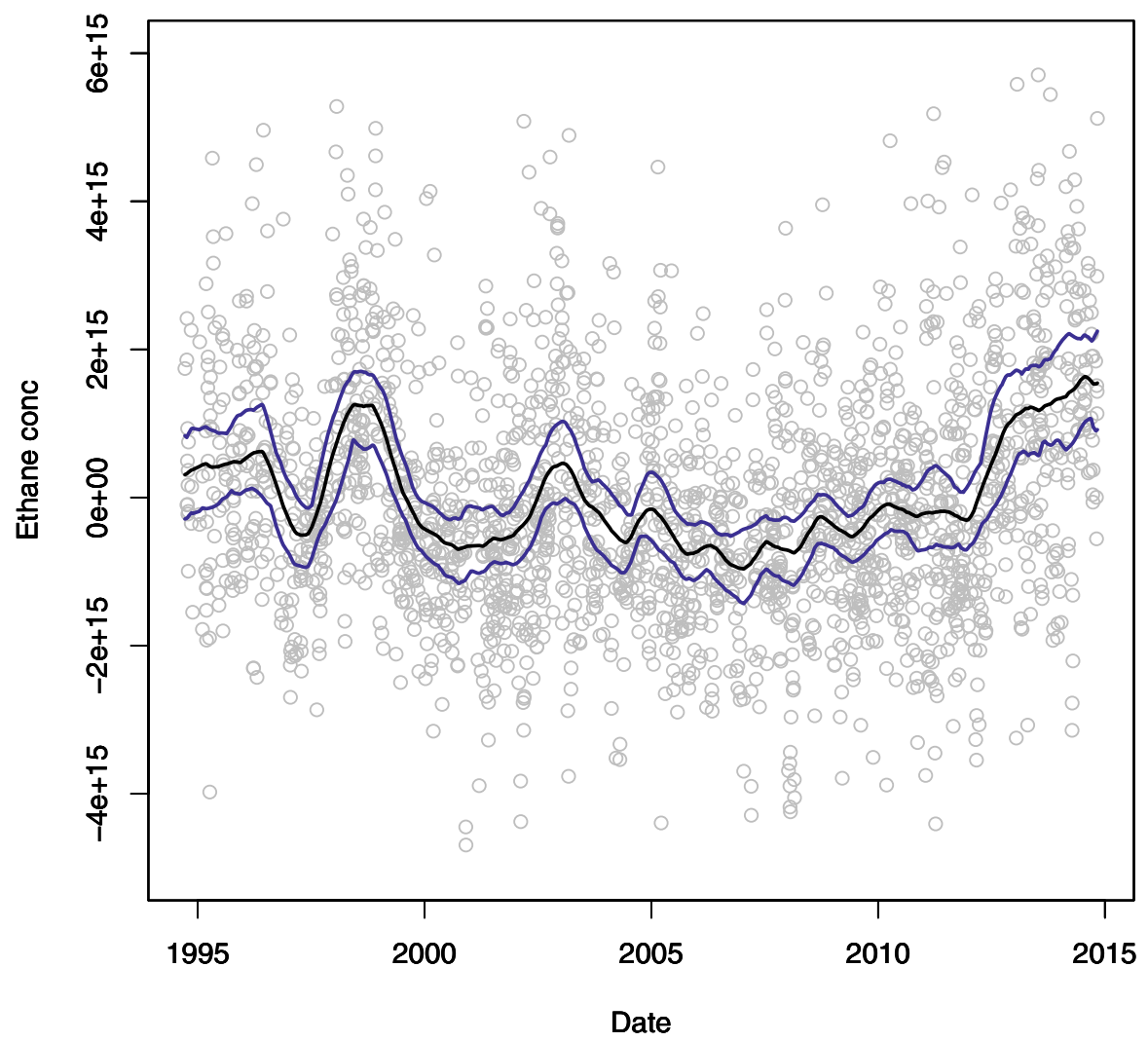

Figure 2.8: $M=4, h=0.03$ 


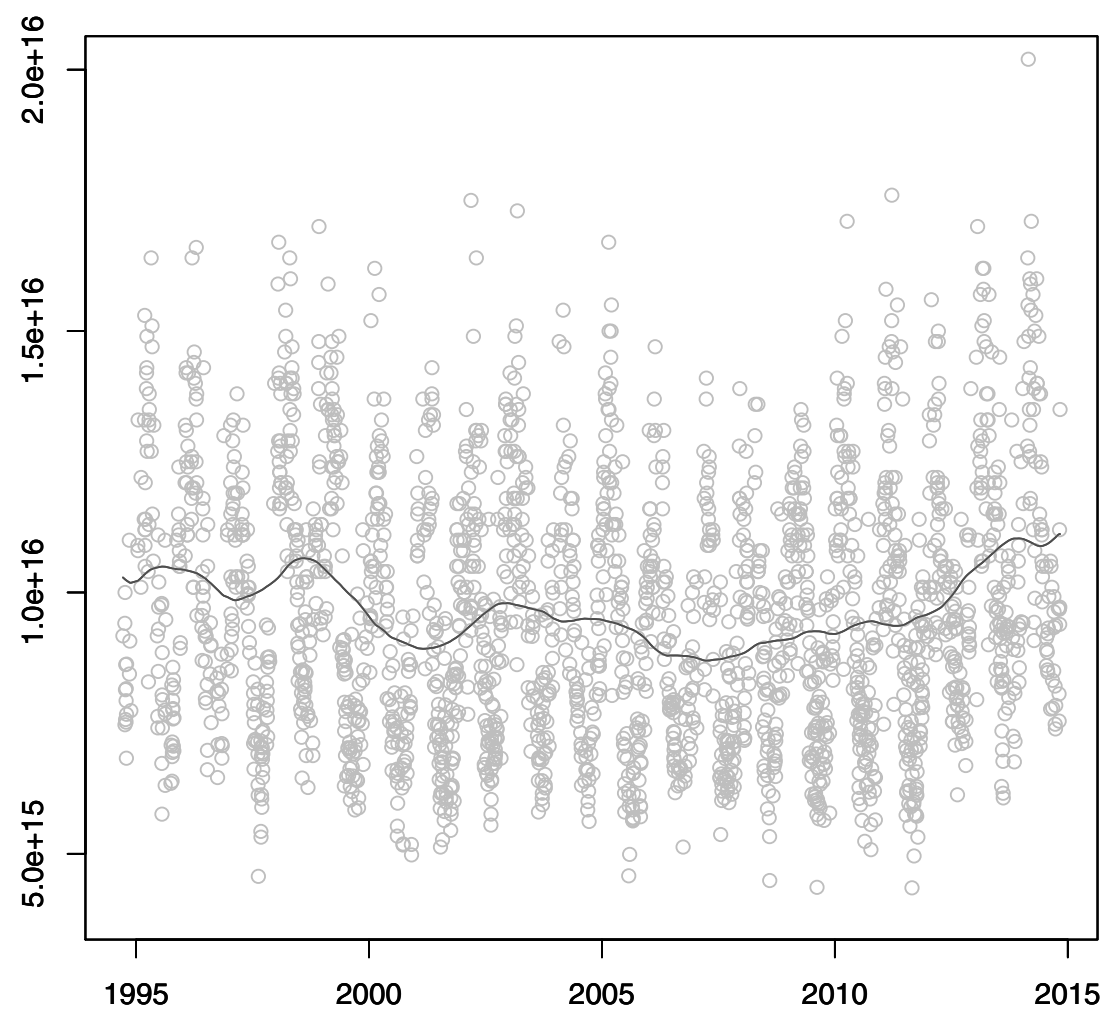

Figure 2.9: $h=0.06$ 



\section{Chapter 3}

\section{Trends in atmospheric time series: an application to ethane}

Understanding the development of trends and identifying trend reversals in decadal time series is becoming more and more important. Many climatological and atmospheric time series are characterized by autocorrelation, heteroskedasticity and seasonal effects. Additionally, missing observations due to instrument failure or unfavorable measurement conditions are common in such series. This is why it is crucial to apply methods which work reliably under these circumstances. The goal of this chapter is to provide a toolbox which can be used to determine the presence and form of changes in trend functions using parametric as well as nonparametric techniques. We consider bootstrap inference on broken linear trends and smoothly varying nonlinear trends. Our autoregressive wild bootstrap approach combined with a seasonal filter, is able to handle all issues mentioned above. We apply our methods to a set of atmospheric ethane series with a focus on the measurements obtained above the Jungfraujoch in the Swiss Alps. Ethane is a good indicator of oil and gas production as well as transport. Its monitoring is therefore crucial for the characterization of air quality and of the transport of tropospheric pollution. ${ }^{1}$

\footnotetext{
${ }^{1}$ This chapter is based on the article $A$ statistical analysis of time trends in atmospheric ethane by Friedrich, M., Beutner, E., Reuvers, H., Smeekes, S., Urbain, J.-P., Bader, W., Franco, B., Lejeune, B. and E. Mahieu, Climatic Change, 162, 1, p. 105-125.
} 



\subsection{Introduction}

In the presence of global climate change, understanding the development of decadal time series becomes more and more relevant. A major part of this understanding lies in the analysis of time trends and the detection of trend reversals. Many tools are available for this purpose in the econometrics and statistics literature. They range from linear trend analysis to more advanced methods such as trends with breaks, polynomial trends, or smooth trends of unspecified form. Estimation of the trend, however, is not enough; it is crucial that with every estimate we can indicate the corresponding uncertainty around it. This is commonly achieved by calculating confidence intervals which enable us to judge the significance of our results.

Climatological data, however, frequently display characteristics which complicate the calculation of such uncertainty measures. These characteristics can include strong seasonality, different degrees of variability in the data, such as significant inter-annual changes, and missing observations due to instrument failures or unfavorable measurement conditions. Therefore, it is important to use methods which provide reliable results even under these circumstances. A prominent example in which the three problematic characteristics arise is atmospheric ethane, when measured with the ground-based Fourier Transform InfraRed (FTIR) technique. It displays strong seasonality, a time-varying variance and, since measurements can only be taken under clear sky conditions, has many missing data points.

It is increasingly popular to resort to bootstrap methods to address these problems. Similar arguments are presented in Gardiner et al. (2008) who propose a method for trend analysis of greenhouse gases. Their approach estimates a linear trend using least squares minimization. Subsequently, to obtain confidence intervals for the slope parameter, they introduce an i.i.d. (independently and identically distributed) bootstrap method.

This combination of linear trend estimation and i.i.d. bootstrap has been applied to investigate trends in various data series; see e.g. De Smedt et al. (2010), Mahieu et al. (2014), Franco et al. (2015) and Hausmann et al. (2016). The latter paper uses the bootstrap method to study trends in atmospheric methane and ethane concentrations measured at Zugspitze and Lauder. They split the sample into two time periods and compare the changes in trends between both periods.

This approach, however, suffers from two major drawbacks. First, in the presence of autocorrelation, the i.i.d. bootstrap method cannot correctly mimic the 
dependence structure of the residuals. Alternative bootstrap methods are available which can solve this problem and they require just minor modifications in implementation. Second, the approach does not provide a formal test if and where the sample should be split and it does not give a measure of uncertainty regarding the location of the obtained break.

The aim of this chapter is to propose a toolbox for trend estimation which addresses these drawbacks. We give an overview of selected statistical and econometric tools designed for the analysis of time trends and trend reversals. We focus on two different, but complimentary approaches which are particularly suited in this context. In the first part, we present a linear trend model which allows for a break at an unknown time point. It provides researchers with a tool to test if there was a break present in the trend and, if so, it additionally gives an estimate of its location with a reliable confidence interval.

In the second part, we move to a more general specification by considering a smoothly varying trend model. This approach is more flexible as it does not impose any assumptions regarding the form of the trend. We propose three additional methods in which we take a closer look at the trend obtained from this approach. First, we identify the locations of (local) extrema, as they are typically associated with trend reversal, and propose a method to construct a confidence interval around the locations. Second, we investigate a method for testing if the trend can be described by a specific parametric form. We test if the trend (possibly in a subsample) can adequately be described by a parametric function, such as a linear one. Third, we provide a test for monotonicity of the trend function on a subsample, which does not require fitting a parametric function, but can directly be applied on the nonparametrically estimated trend.

In both parts, we suggest the use of bootstrap methods. They can be used for the construction of confidence intervals as well as to obtain critical values for the tests. We advocate the use of a specific bootstrap method - the autoregressive wild bootstrap - which is applicable to correlated data. Its second advantage over many other bootstrap methods is that it can easily be applied to data series with missing observations.

All methods are used to study the atmospheric ethane $\left(\mathrm{C}_{2} \mathrm{H}_{6}\right)$ burden with the help of a set of six time series. The series consist of daily ethane columns (i.e. the number of molecules integrated between the ground and the top of the atmosphere in a column of a given area, e.g. a square centimeter) obtained from ground-based 
FTIR measurements. In particular, we will focus on the Jungfraujoch ethane measurements.

The structure of the chapter is as follows. In Section 2, we present the data. Section 3 introduces the linear trend approach. We show how to determine the break point, how to estimate the trend and how to construct confidence intervals. Section 4 continues with the nonparametric trend model, its estimation and confidence intervals. In this section, we also present how to conduct inference on the shape of the nonparametric trends. At the end of each section, the respective methods are applied to the data. Section 5 concludes.

\subsection{The ethane data}

Ethane is the most abundant non-methane hydrocarbon in the Earth's atmosphere and has several properties which make it important to study. First, ethane is an indirect greenhouse gas influencing the atmospheric lifetime of methane. It degrades by reacting with the same oxidizer which is needed for the degradation of other major greenhouse gases like e.g. methane. Molecules of the oxidizer which are occupied by ethane are not available for the destruction of other pollutants. Second, ethane is an important precursor of tropospheric ozone. It contributes to the formation of ground level ozone $\left(\mathrm{O}_{3}\right)$ which is a major pollutant affecting the air quality. While ozone in higher levels of the atmosphere protects us from the Sun's harmful ultraviolet rays, ground level ozone damages ecosystems and has adverse affects on the human body. Third, ethane emissions can be used as a measure of methane emissions. Both gases are co-emitted from the oil and gas exploitation, while ethane does not have significant natural sources, methane is released in the atmosphere by both natural activities and human-induced ones. This makes it hard to measure the fraction of methane released by the oil and gas sector. An estimate of this fraction can be provided with the help of ethane measurements. Its monitoring is therefore crucial for the characterization of air quality and of the transport of tropospheric pollution. The main sources of ethane are located in the Northern hemisphere, and the dominating emissions are associated with production and transport of oil and natural gas.

We illustrate our proposed methods by analyzing time trends in six series of atmospheric ethane measurements. In the text we focus, however, on one series which is derived from observations performed at the Jungfraujoch station in the Swiss Alps. It is located on the saddle between the Jungfrau and the Mönch, 
located at $46.55^{\circ} \mathrm{N}, 7.98^{\circ} \mathrm{E}, 3580 \mathrm{~m}$ altitude. Results obtained from the other five data series will be summarized in tables and related graphs can be found in the Appendix.

The Jungfraujoch ethane series has been studied in detail in Franco et al. (2015). The paper also provides further details on the ground-based station at Jungfraujoch and on how the measurements are obtained. We focus on this series, because measurement conditions are very favorable at this location due to high dryness and low local pollution. It is a time series consisting of daily ethane columns recorded under clear-sky conditions between September 1994 and August 2014 with a total of 2260 data points. For the location and sample size of the other five series we refer to the first column of Table 3.1 in the next section.

Giving an indication of the severity of the missing data problem present in this series, the Jungfraujoch series contains an average number of data points per year of 112.6. The situation is similar in the other series. In addition to missing data points, the series also exhibit seasonality. Ethane degrades faster under warm weather conditions than in cold temperature and therefore, the measurements display local peaks every winter period.

All results in the following sections are obtained using $B=999$ replications of the bootstrap procedure. Whenever confidence intervals or bands are given, these are for a nominal coverage level of $95 \%$.

\subsection{The linear trend approach}

We consider a linear trend model including the possibility of a break in the trend line. At the breakpoint, the slope of the trend function is allowed to change. The intercept change is restricted in such a way that there will be no discontinuity at the break, i.e. no jump between the consecutive linear functions. In this section, we first present the model specifications. Second, we show how the model can be estimated. Third, the bootstrap method for the construction of confidence intervals is introduced. The final part applies the proposed approach to the data.

\subsubsection{A broken trend model}

We consider a linear regression with the following specification. For $t=1, \ldots, n$,

$$
y_{t}=\alpha+\beta t+\delta D_{t, T_{1}}+F_{t}+u_{t},
$$


where

$$
D_{t, n_{1}}= \begin{cases}0 & \text { if } t \leq T_{1} \\ t-n_{1} & \text { if } t>T_{1}\end{cases}
$$

In this specification, $\left\{y_{t}\right\}$ is the time series of interest, $t$ refers to the time trend with slope parameter $\beta$ and $\alpha$ is the intercept. The break occurs at time $T_{1}$. The variable $D_{t, T_{1}}$ is a dummy variable which induces a change in the slope coefficient from $\beta$ to $(\beta+\delta)$ after $T_{1}$. At the same time, the intercept of the trend function changes in such a way that the successive linear components are joined at the breakpoint. Additionally, the term $F_{t}$ captures the intra-annual variability, which is present in almost all climate time series, with the help of Fourier terms

$$
F_{t}=\sum_{j=1}^{M} a_{j} \cos (2 j \pi t)+b_{j} \sin (2 j \pi t) .
$$

This specification of the seasonal variability is widely used when estimating trends in atmospheric gases, see e.g. Gardiner et al. (2008), Franco et al. (2015) and Franco et al. (2016). It has been shown in these papers that the variability is well captured by the inclusion of three sine and cosine terms. We follow the same approach and will consider equation (3.3) with $M=3$ in the remainder of the chapter. The errors $\left\{u_{t}\right\}$ are allowed to be serially correlated and to exhibit changes in variance. The goal is to estimate $(\alpha, \beta, \delta)$. The parameters of equation (3.3) will also be estimated during the process and we will denote the fitted version by $\hat{F}_{t}$. To formalize the above mentioned missing data problem, we note that we observe a part of the observations $y_{1}, \ldots, y_{n}$. We define a binary variable $M_{t}$ as

$$
M_{t}=\left\{\begin{array}{ll}
1 & \text { if } y_{t} \text { is observed } \\
0 & \text { if } y_{t} \text { is missing }
\end{array} \quad t=1, \ldots, n .\right.
$$

We assume that the missing pattern, characterized by $\left\{M_{t}\right\}$, is independent of the observations and can be weakly dependent with the dependence decaying to zero over time. It is reasonable to assume that the pattern of the missing data points in the case of FTIR measurements follows these assumptions. A more detailed discussion on this issue can be found in Friedrich et al. (2019).

Before we continue with a description of the estimation procedure, we give a formal test to determine whether a model with one break is preferred over a simple 
linear trend model. It is based on Bai and Perron (1998). Let $\Lambda=[\lambda n,(1-\lambda) n]$, with $0<\lambda<1 / 2$. By using $\Lambda$ as a set for possible break dates, we ensure that a candidate break, $T_{c}$, is neither too close to the beginning of the sample nor too close to the end. Otherwise, the number of observations on each side of the candidate break would not be sufficient for further estimation. We use the test statistic

$$
\begin{aligned}
S_{n}= & \min _{\alpha, \beta, F_{t}} \sum_{t=1}^{n} M_{t}\left(y_{t}-\alpha-\beta t-F_{t}\right)^{2} \\
& -\inf _{n_{c} \in \Lambda} \min _{\alpha, \beta, \delta, F_{t}} \sum_{t=1}^{n} M_{t}\left(y_{t}-\alpha-\beta t-\delta D_{t, T_{c}}-F_{t}\right)^{2},
\end{aligned}
$$

where we compare the sum of squared residuals of a model without break to the lowest sum of squared residuals of a model including one break. It is a formal test of the pair of hypothesis $H_{0}: \delta=0$ versus $H_{1}: \delta \neq 0$, for every possible break point $T_{c}$. For high values of $S_{n}$, there is evidence that the model with break fits the data better and we reject $H_{0}$; for low values we fail to reject it. Given a significance level of the test, the critical value of the test determines the cut-off point and we obtain it using the autoregressive wild bootstrap. Further details on this bootstrap method are postponed to Section 3.3.3.

\subsubsection{Estimation}

The first step in the procedure is to estimate the break date. Subsequently, given the estimated break date, the parameter estimates of interest are determined. Given a candidate break date $n_{c}$, estimates of $\left(\alpha, \beta, \delta, F_{t}\right)$ are obtained by minimizing the following sum of squared residuals

$$
\left(\hat{\alpha}_{T_{c}}, \hat{\beta}_{T_{c}}, \hat{\delta}_{T_{c}}, \hat{F}_{t, T_{c}}\right)=\underset{\alpha, \beta, \delta, F_{t}}{\operatorname{argmin}} \sum_{t=1}^{n} M_{t}\left(y_{t}-\alpha-\beta t-\delta D_{t, T_{c}}-F_{t}\right)^{2},
$$

where we make explicit that these estimates are for a candidate break date and not the final parameter estimates by using a subscript $T_{c}$. Let us come back to the two step procedure. For the first step, which is estimating the break location, we need to construct such a sum of squared residuals for every admissible break date candidate $T_{c}$. The minimum over all possible candidates gives us the estimated break date. Let $\Lambda$ be the same set as in the previous section and consider the 
following minimization problem

$$
\hat{T}_{1}=\underset{T_{c} \in \Lambda}{\operatorname{argmin}} \sum_{t=1}^{n} M_{t}\left(y_{t}-\hat{\alpha}_{T_{c}}-\hat{\beta}_{T_{c}} t-\hat{\delta}_{T_{c}} D_{t, T_{c}}-\hat{F}_{t, T_{c}}\right)^{2},
$$

where $\left(\hat{\alpha}_{T_{c}}, \hat{\beta}_{T_{c}}, \hat{\delta}_{T_{c}}, \hat{F}_{t, T_{c}}\right)$ are determined as described above. Once we have obtained the estimate of the break date, $\hat{n}_{1}$, we construct the corresponding leastsquares parameter estimates in the second step. To be consistent with the notation, these will be denoted as $\left(\hat{\alpha}, \hat{\beta}, \hat{\delta}, \hat{F}_{t}\right)=\left(\hat{\alpha}_{\hat{T}_{1}}, \hat{\beta}_{\hat{T}_{1}}, \hat{\delta}_{\hat{T}_{1}}, \hat{F}_{t, \hat{T}_{1}}\right)$.

\subsubsection{Confidence intervals}

Given the parameter estimates and the estimated break location, we need to rely on a measure of uncertainty to be able to judge the significance of our findings. A major difficulty with climate time series is the presence of serial correlation of the residuals from our estimated model. An additional complication arises due to the fact that these time series are mostly made of observations which are unequally spaced over the sample period $t=1, \ldots, n$. To overcome these difficulties, we propose a bootstrap method which has been established in the econometrics and statistics literature as being very successful and providing accurate confidence intervals even in small samples. It solves both, the problem caused by serial correlation and the unequally spaced data. In addition, it protects against possible changes in variance of the residuals.

To form bootstrap samples, the standard bootstrap method - the i.i.d. bootstrap - draws randomly and with replacement from the residuals and, thereby, destroys both the dependence structure and possible time variations in the variance. Due to these reasons, the bootstrap sample will not correctly reflect the original series of residuals and the general principle, on which bootstrap methods are based, is violated. The goal, therefore, is to construct bootstrap errors which have the same pattern of correlation, variance changes and missing data as the original set of residuals. The autoregressive wild bootstrap, which is proposed in the context of nonparametric trend estimation in Friedrich et al. (2019), is to our knowledge the best way to achieve this goal. In particular, in the presence of serial correlation compared to its competitors - sieve or block bootstrap methods - it holds a clear advantage as it has a natural way of handling missing data. No adjustments are needed for it to reproduce the missing data pattern in the original sample. 
In the remainder of this section, we present details on this bootstrap method by giving the bootstrap algorithm for the case of the break location. We then explain how it can easily be adapted to form confidence intervals for the parameter estimates of slope and intercept. To determine confidence intervals for the break date estimate, we use the following bootstrap algorithm:

Algorithm 3.1 (Autoregressive Wild Bootstrap - Break location).

1. Calculate residuals from the estimation of Model (3.1). Impose a break at $\hat{n}_{1}$. For $t=1, \ldots, n$,

$$
\hat{u}_{t}=M_{t}\left(y_{t}-\hat{\alpha}-\hat{\beta} t-\hat{\delta} D_{t, \hat{T}_{1}}-\hat{F}_{t}\right)
$$

2. For $0<\gamma<1$, generate $\nu_{1}^{*}, \ldots, \nu_{n}^{*}$ as i.i.d. $\mathcal{N}\left(0,1-\gamma^{2}\right)$ and let $\xi_{t}^{*}=\gamma \xi_{t-1}^{*}+\nu_{t}^{*}$ for $t=2, \ldots, n$. Take $\xi_{1}^{*} \sim \mathcal{N}(0,1)$ to ensure stationarity of $\left\{\xi_{t}^{*}\right\}$.

3. Calculate the bootstrap errors $u_{t}^{*}=M_{t} \xi_{t}^{*} \hat{u}_{t}$ and create the bootstrap sample as

$$
y_{t}^{*}=M_{t}\left(\hat{\alpha}+\hat{\beta} t+\hat{\delta} D_{t, \hat{T}_{1}}+\hat{F}_{t}+u_{t}^{*}\right)
$$

for $t=1, \ldots, n$, using the same estimated coefficients as in Step 1.

4. Determine $\hat{T}_{1}^{*}$ from $y_{t}^{*}$ as in (3.7) and store the estimates.

5. Repeat Steps 2 to $4 B$ times to obtain the bootstrap distribution of $\hat{T}_{1}^{*}$.

Note that in Step 2 we generate $\left\{\xi_{t}^{*}\right\}$ for all $t=1, \ldots, n$, although in Step 3 we construct bootstrap errors and subsequently, bootstrap observations only when there exists an actual data point. This is what the multiplication by $M_{t}$ in Step 3 ensures. The bootstrap sample thus correctly reflects the missing pattern present in the data.

In Step 2 of the above algorithm, the autoregressive coefficient $\gamma$ has to be chosen. To obtain an asymptotically valid bootstrap method, that is, bootstrap confidence intervals whose confidence level approaches $(1-\alpha)$ in the limit, Friedrich et al. (2019) show that $\gamma$ should tend to 1 as the sample size $n$ increases. Friedrich et al. (2019) suggest to achieve this by setting $\gamma=\theta^{1 / l}$ with $l=1.75 n^{1 / 3}$ and $\theta=0.1$.

The confidence intervals are then determined by the $\alpha / 2$ quantile and $(1-\alpha / 2)$ quantile of the bootstrap distribution. In analogy to this algorithm, we can obtain 
confidence intervals for the parameter estimates by adjusting Step 4. Given the estimated break location $\hat{n}_{1}$, we estimate Model (3.1) from $y_{t}^{*}$, thereby imposing the same break as found in the data. The bootstrap estimates $\hat{\alpha}^{*}, \hat{\beta}^{*}$ and $\hat{\delta}^{*}$ are stored and the corresponding bootstrap distributions and quantiles are constructed in the same way as in the previously described case.

The same bootstrap method is also used to obtain critical values for the test statistic $S_{n}$ as in (3.5). The approach is very similar to above. In this application, however, the bootstrap series are constructed under the null hypothesis of no break, meaning that a linear trend model without break is estimated from the data in Step 1 and then used to form residuals. Once the bootstrap series is constructed using the linear trend from Step 1, the bootstrap version of the test statistic $S_{n}^{*}$ is determined.

Algorithm 3.2 (Autoregressive Wild Bootstrap - Break test).

1. Calculate residuals from the estimation of Model (3.1) with $\delta=0$. For $t=1, \ldots, n$,

$$
\hat{u}_{t}=M_{t}\left(y_{t}-\hat{\alpha}-\hat{\beta} t-\hat{F}_{t}\right)
$$

2. Identical to Step 2 of Algorithm 3.1.

3. Calculate the bootstrap errors $u_{t}^{*}=M_{t} \xi_{t}^{*} \hat{u}_{t}$ and create the bootstrap sample as

$$
y_{t}^{*}=M_{t}\left(\hat{\alpha}+\hat{\beta} t+\hat{F}_{t}+u_{t}^{*}\right)
$$

for $t=1, \ldots, n$, using the same estimated coefficients as in Step 1.

4. Obtain $S_{n}^{*}$ from $y_{t}^{*}$ as in equation (3.5) and store the result.

5. Repeat Steps 2 to $4 B$ times to obtain the bootstrap distribution of $S_{n}^{*}$.

Since the test is rejected for large values of the test statistic $S_{n}$, we use the $(1-\alpha)$ quantile of the ordered bootstrap statistics as critical value for the break test.

\subsubsection{Data application}

We now present results of an application of the tools to the ethane time series. The statistical tests which help us to decide whether a break is present in the trend 


\begin{tabular}{lccccc}
\hline \hline & $n$ & $p$-value & $\mathcal{F}_{n}$ & $C V$ & Discussed in \\
\hline Jungfraujoch & 2260 & 0.0000 & $6.22 \times 10^{32}$ & $0.52 \times 10^{32}$ & Franco et al. (2015) \\
Boulder & 164 & 0.0225 & $1.71 \times 10^{32}$ & $1.27 \times 10^{32}$ & Franco et al. (2016) \\
Eureka & 725 & 0.0049 & $8.79 \times 10^{31}$ & $5.16 \times 10^{31}$ & Franco et al. (2016) \\
Lauder & 2550 & 0.0248 & $2.82 \times 10^{31}$ & $2.30 \times 10^{31}$ & Zeng et al. (2012) \\
Thule & 814 & 0.0000 & $1.99 \times 10^{32}$ & $0.75 \times 10^{32}$ & Franco et al. (2016) \\
Toronto & 1399 & 0.0000 & $1.93 \times 10^{33}$ & $2.84 \times 10^{32}$ & Franco et al. (2016) \\
\hline
\end{tabular}

Table 3.1: Results of the break tests $(\pi=0.1)$

line give strong evidence for the alternative hypothesis in all series, thus indicating that a model with a break should be preferred over a simple linear trend model. Detailed results of the tests for all six time series are summarized in Table 3.1.

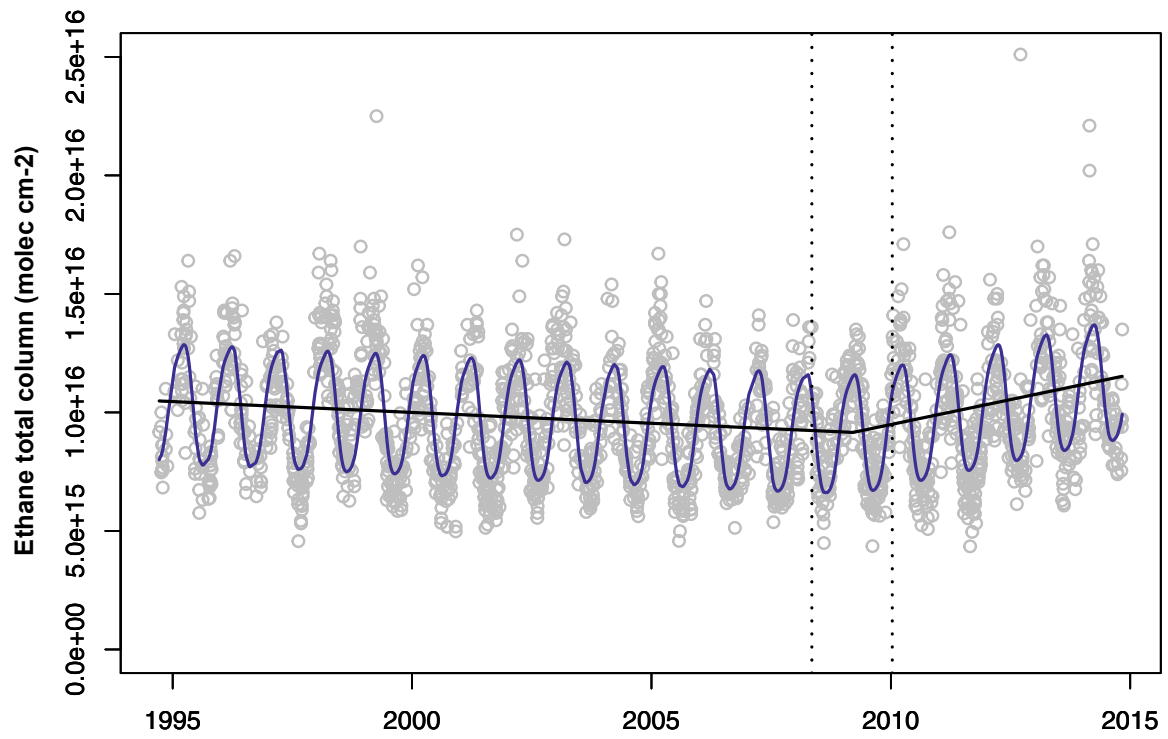

Figure 3.1: Breakpoint estimate and confidence interval for Jungfraujoch

We find a breakpoint in the Jungfraujoch ethane column series in the beginning of 2009. This is in line with the findings of Franco et al. (2015). In addition to the point estimate of the break location, which is 2009.21, the autoregressive wild bootstrap enables us to find a confidence interval for this break ranging from 2008.35 to 2010.03. The results are summarized graphically in Figure 3.1 which plots the ethane time series (gray circles), the seasonal fit of three Fourier terms 
(blue) as well as the estimated broken trend (black). The confidence interval for the break location is indicated by the two dotted vertical lines. Before the break, there is a significant downward trend, which turns into an upward trend after the break. The slope after the break is positive. The estimates are presented in Table 3.2 for all six series. In addition, Figure 3.4 in the Appendix plots these results in the same fashion as in Figure 3.1.

As mentioned by Franco et al. (2015), the initial downward trend can be explained by a general emission reduction since the mid 1980's, of the fossil fuel sources in the Northern Hemisphere. The upward trend seems to be a more recent phenomenon. There are studies that attribute it to the recent growth in the exploitation of shale gas and tight oil reservoirs, taking place in North America, see e.g. Vinciguerra et al. (2015), Franco et al. (2016) and Helmig et al. (2016). The significant negative coefficients before and after the break in Table 3.2 indicate that Lauder is not yet impacted by the recent increase of ethane in the Northern hemisphere, further from the main emission sources. Lauder is the only site in the data set which is located in the Southern hemisphere. Indeed, ethane has a mean atmospheric lifetime of 2 months, significantly shorter than the time needed to mix air between both hemispheres.

\subsection{A smooth trend function}

While the previous analysis restricted the trend to a (broken) linear form, in this section, we look at an alternative trend model. It is more flexible in the sense that it does not require us to specify a specific form of the trend in advance. This section is divided into two main parts. The first part follows the structure of the previous section. In the second part, we propose several tests which allow us to further investigate the obtained results.

\subsubsection{The nonparametric trend model}

As an alternative model specification, we look at the following nonparametric trend model

$$
y_{t}=g(t / n)+F_{t}+u_{t} \quad t=1, \ldots, n
$$

where $F_{t}$ is the same series of Fourier terms as in equation (3.3). As above, we only observe a part of the data. We allow for heteroskedasticity and additionally, 


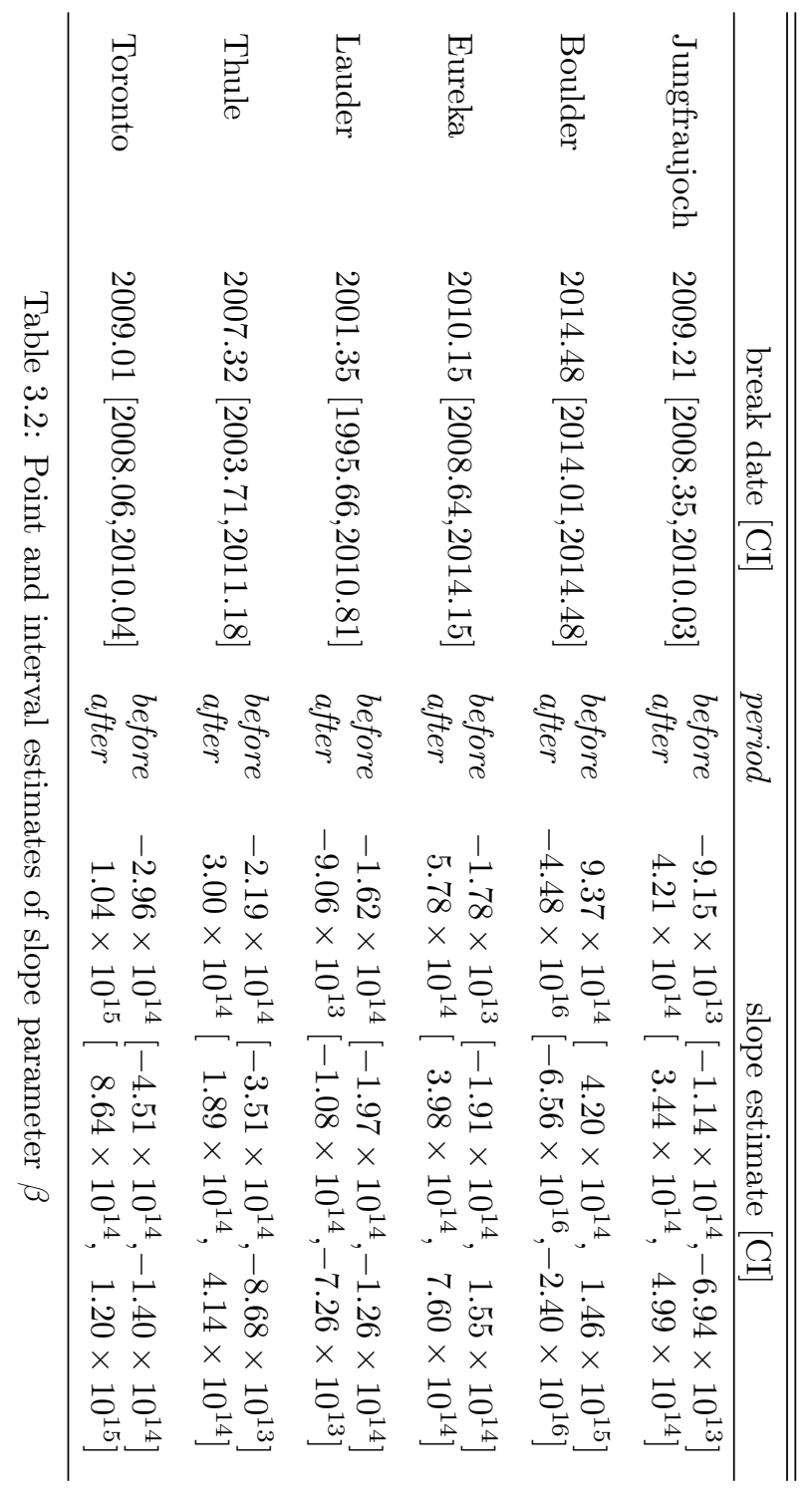


serial correlation is captured by allowing $\left\{u_{t}\right\}$ to be weakly dependent over time with the dependence dying out. For details on the explicit assumptions we refer to Friedrich et al. (2019). The nonparametric trend specification has the advantage that no particular form of the trend function has to be imposed. While in the previous section, we assume that the data are following Model (3.1) - a linear trend with one break - we are more flexible regarding the trend function in this case. The function $g(\cdot)$ can be of any form as long as it is smooth in the sense that it is twice continuously differentiable with bounded derivatives. Estimation is done using a two-stage procedure to first eliminate the seasonal variability and then estimate the trend function nonparametrically. Inference on the estimated trend is conducted using the autoregressive wild bootstrap to construct pointwise intervals in a similar fashion as above. Subsequently, we apply a simple three step procedure to find simultaneous confidence bands based on the pointwise intervals. Details are given in the next two subsections.

\section{Estimation}

The main goal is to estimate the trend function $g(\cdot)$ and to determine the uncertainty around this estimate. To achieve this goal we propose a two-stage estimation procedure. In the first stage, $y_{t}$ is regressed on the Fourier terms and in the second stage, the residuals from the first stage are used to estimate the trend nonparametrically. Denote by $\hat{u}_{t}$ the residuals from a regression of $y_{t}$ on the Fourier terms so that $\hat{u}_{t}=M_{t}\left(y_{t}-\hat{F}_{t}\right)$. To estimate the trend function from $\left\{\hat{u}_{t}\right\}$, we apply a nonparametric kernel estimator: the local constant Nadaraya-Watson estimator. It is found by minimizing a weighted sum of squares with respect to $g(\cdot)$. This is done for every point $t=1, \ldots, n$. As is standard with this approach, we map the points into the interval $(0,1)$. Thus, for $\tau \in(0,1)$, we obtain:

$$
\begin{aligned}
\hat{g}(\tau) & =\underset{g(\tau)}{\arg \min } \sum_{t=1}^{n} K\left(\frac{t / n-\tau}{h}\right) M_{t}\left\{\hat{u}_{t}-g(\tau)\right\}^{2} \\
& =\left[\sum_{t=1}^{n} K\left(\frac{t / n-\tau}{h}\right) M_{t}\right]^{-1} \sum_{t=1}^{n} K\left(\frac{t / n-\tau}{h}\right) M_{t} \hat{u}_{t},
\end{aligned}
$$

where $K(\cdot)$ is a kernel function and $h>0$ is the bandwidth. We propose to use the Epanechnikov kernel given by $K(x)=\frac{3}{4}\left(1-x^{2}\right) \mathbb{1}_{\{|x| \leq 1\}}$. The parameter $h$ is a smoothing parameter. Large bandwidths produce a very smooth estimate, while for small bandwidth, the trend becomes less smooth. Bandwidth selection 
is an important aspect for this type of estimation. Therefore, we propose using a data-driven selection method valid for time series applications, called modified cross-validation (MCV). It is based on the original criterion proposed in $\mathrm{Chu}$ and Marron (1991):

$$
C V_{k}(h)=\frac{1}{n} \sum_{t=1}^{n} M_{t}\left(\hat{g}_{k, h}\left(\frac{t}{n}\right)-\hat{u}_{t}\right)^{2}
$$

where

$$
\hat{g}_{k, h}(t / n)=\frac{(n-2 k-1)^{-1} \sum_{t:|t-\tau n|>k} K\left(\frac{t / n-\tau}{h}\right) M_{t} \hat{u}_{t}}{(n-2 k-1)^{-1} \sum_{t:|t-\tau n|>k} K\left(\frac{t / n-\tau}{h}\right) M_{t}}
$$

leaves out the $(2 k+1)$ observations receiving the highest weight. The optimal bandwidth is found by minimizing this criterion with respect to $h$. We elaborate on the practical implementation and give an example during the application in Section 3.4.1.

\section{Confidence intervals}

As we argued above, to merely rely on point estimates of the trend function to draw conclusions about the underlying trend is not sufficient. Confidence intervals are needed to say more about the significance of the trend. In this setting, the object of interest is the trend function as a whole and there is no single parameter which summarizes certain features of this function - like the slope coefficient in the previous approach. Therefore, it is crucial to have a tool to judge the significance over more than one time point, preferably, over the whole sample. Confidence intervals which allow for this could be used, for example, to assess whether there has been a significant non-zero upward or downward trend. This could be checked by trying to place a straight line as a trend function and then observing if this line can be completely embedded within the confidence intervals.

Confidence bands, where the coverage simultaneously holds over more than one point or even the whole sample can be constructed from pointwise confidence intervals. We therefore first propose a method to construct pointwise confidence intervals for the nonparametric trend estimate. Second, we suggest a three-step procedure to transform pointwise intervals into simultaneous confidence bands. For this type of model, it has been shown in the statistical literature that boot- 
strap methods are a reliable tool to conduct inference (see e.g. Bühlmann (1998), Neumann and Polzehl (1998)). We again propose to use the autoregressive wild bootstrap. A minor adjustment compared to the above algorithm has to be made. Therefore, we give the full bootstrap algorithm again, for completeness.

Algorithm 3.3 (Autoregressive Wild Bootstrap - Nonparametric trend).

1. Let $\tilde{g}(\cdot)$ be defined as in $(3.9)$, but using bandwidth $\tilde{h}$. Obtain residuals

$$
\hat{\epsilon}_{t}=M_{t}\left(\hat{u}_{t}-\tilde{g}(t / n)\right), \quad t=1, \ldots, n
$$

2. Identical to Step 2 of Algorithms 3.1 and 3.2.

3. Calculate the bootstrap errors $\epsilon_{t}^{*}$ as $\epsilon_{t}^{*}=M_{t} \xi_{t}^{*} \hat{\epsilon}_{t}$ and generate the bootstrap observations by

$$
\hat{u}_{t}^{*}=M_{t}\left(\tilde{g}(t / n)+\epsilon_{t}^{*}\right), \quad t=1, \ldots, n,
$$

where $\tilde{g}(t / n)$ is the same estimate as in the first step.

4. Obtain the bootstrap estimator $\hat{g}^{*}(\cdot)$ as defined in (3.9) using the bootstrap series $\hat{u}_{t}^{*}$, with the same bandwidth $h$ as used for the original estimate $\hat{g}(\cdot)$.

5. Repeat Steps 2 to 4 a total of $B$ times and let

$$
\hat{q}_{\alpha}(\tau)=\inf \left\{u ; P^{*}\left[\hat{g}^{*}(\tau)-\tilde{g}(\tau) \leq u\right] \geq \alpha\right\}
$$

denote the $\alpha$-quantile of the $B$ centered bootstrap statistics $\hat{g}^{*}(\tau)-\tilde{g}(\tau)$. These bootstrap quantiles are used to construct confidence bands as described below.

Note that in Step 1 of the above algorithm, a different bandwidth is used to perform the nonparametric estimation. We suggest to use the larger bandwidth $\tilde{h}=0.5 h^{5 / 9}$. This produces an oversmoothed estimate as starting point for the bootstrap procedure. Details on this issue as well as the asymptotic validity of the bootstrap method are shown in Friedrich et al. (2019).

Using Algorithm 3.3, we can obtain pointwise bootstrap confidence intervals for the nonparametric trend $g(\tau)$ with a confidence level of $(1-\alpha)$. They are denoted by $I_{n, \alpha}^{(p)}(\tau)$ and are constructed such that

$$
\liminf _{n \rightarrow \infty} \mathbb{P}\left(g(\tau) \in I_{n, \alpha}^{(p)}(\tau)\right) \geq 1-\alpha \quad \tau \in(0,1) .
$$


Using the $\alpha$-quantiles from Step 5 of the bootstrap algorithm, we can generate such pointwise intervals as

$$
I_{n, \alpha}^{(p)}(\tau)=\left[\hat{g}(\tau)-\hat{q}_{1-\alpha / 2}, \hat{g}(\tau)-\hat{q}_{\alpha / 2}\right]
$$

These intervals are constructed separately for each point $\tau$. Therefore, a link over time cannot be established with these intervals. Many interesting research questions, like whether a coefficient remains zero over the whole period or whether there was an upward trend over a certain period of time, cannot be answered with pointwise confidence intervals. The concept of simultaneous confidence bands can be used to answer these questions. In the same notation, the following asymptotic bands are simultaneous over the whole sample

$$
\liminf _{n \rightarrow \infty}\left[\mathbb{P}\left(g(\tau) \in I_{\alpha}(\tau) \quad \tau \in(0,1)\right)\right]=1-\alpha
$$

Practical implementation follows a three-step procedure which was first presented in this context by Bühlmann (1998). It is a search based on the ordered deviations, $\hat{g}^{*}(\tau)-\tilde{g}(\tau)$, of bootstrapped estimates from the original estimate. The first step is to construct pointwise quantiles in the same way as described in Step 5 of Algorithm 3.3. Formally, the three steps are:

1. For all $\tau \in(0,1)$, obtain pointwise quantiles, $\hat{q}_{\alpha_{p} / 2}(\tau), \hat{q}_{1-\alpha_{p} / 2}(\tau)$ for varying $\alpha_{p} \in[1 / B, \alpha]$.

2. Choose $\alpha_{s}$ as

$$
\alpha_{s}=\underset{\alpha_{p} \in[1 / B, \alpha]}{\arg \min }\left|\mathbb{P}^{*}\left[\hat{q}_{\alpha_{p} / 2}(\tau) \leq \hat{g}^{*}(\tau)-\tilde{g}(\tau) \leq \hat{q}_{1-\alpha_{p} / 2}(\tau) \forall \tau \in(0,1)\right]-(1-\alpha)\right|
$$

3. Construct the simultaneous confidence bands as

$$
I_{\alpha_{s}}(\tau)=\left[\hat{g}(\tau)-\hat{q}_{1-\alpha_{s} / 2}(\tau), \hat{g}(\tau)-\hat{q}_{\alpha_{s} / 2}(\tau)\right] \quad \tau \in(0,1)
$$

In the second step of this procedure, a pointwise error $\alpha_{s}$ is found for which a fraction of approximately $(1-\alpha)$ of all centered bootstrap estimates falls completely within the resulting confidence intervals. This value $\alpha_{s}$ is then fixed and the resulting pointwise confidence intervals with coverage $\left(1-\alpha_{s}\right)$ become simultaneous confidence bands with coverage $(1-\alpha)$. 


\section{Data application}

We now apply this procedure. In order to estimate the trend function, we first calculate the residuals from a regression of the ethane data on three Fourier terms. From the residuals we estimate the trend function nonparametrically using the local constant kernel estimator with the Epanechnikov kernel. Bandwidth selection using the Modified Cross Validation (MCV) approach of Chu and Marron (1991) with $k=5$ gives a bandwidth of $h=0.03$.

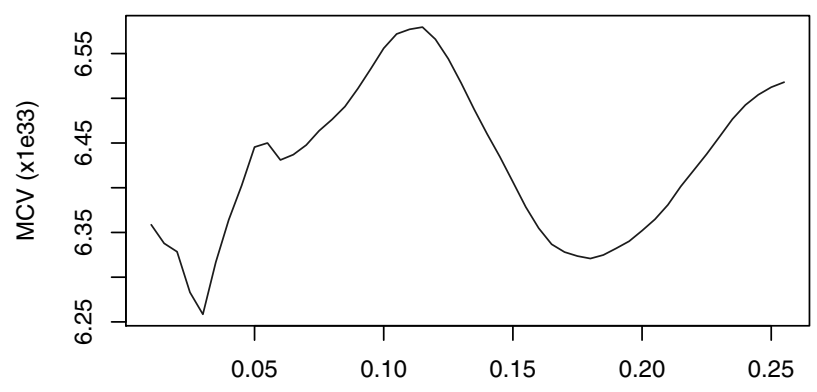

Figure 3.2: Modified Cross Validation Criterion for a range of bandwidths)

As bandwidth selection is an important part of this procedure, we would like to highlight that the MCV procedure has to be applied with care. The range of possible bandwidths over which we minimize the criterion can have a major effect on the resulting optimal bandwidth. The criterion function can have multiple local minima or, if the values contained in our range are too small, the function can be monotonically increasing such that we always select the smallest possible bandwidth. We allow for values between 0.01 and 0.25 in steps of 0.005 . This yields a total of 49 possible bandwidths. To illustrate, we plot the criterion as a function of the bandwidth in Figure 3.2. We can see a global minimum at 0.03 which is the bandwidth we select. A second (local) minimum can be observed at 0.065 and a third minimum lies at 0.185 . Table 3.3 in the Appendix gives an overview of bandwidth selection performed for the other series. In case of the Jungfraujoch data, we decide to select the global minimum, since the resulting trend curve gives a reasonably smooth estimate which allows us to detect interesting features of the trend. Note that the main conclusions we draw below hold with both candidate bandwidths. 
Figure 3.3 plots the seasonally adjusted data points and the nonparametric trend with the $95 \%$ simultaneous confidence bands. If we follow the movements of the trend curve, we see local peaks around the years of 1998 and 2002-2003, which were not visible in the previous analysis. The capture of these two events is possible thanks to the flexibility of the nonparametric approach, since it does not impose the trend to consist of two linear trend lines which is crucial in the parametric procedure. The peaks can be attributed to boreal forest fires which were taking place mainly in Russia during both periods. Geophysical studies have investigated these events in association with anomalies in carbon monoxide emissions (Yurganov et al., 2004, 2005). In such fires, carbon monoxide is coemitted with ethane, such that these events are likely explanations for the peaks we observe.

We nevertheless observe a significant upward trend towards the end of the sample period as in the parametric analysis. Looking at the confidence bands over the last period - starting in 2009 - it is impossible to completely embed a horizontal line into the bands, signaling strong evidence of a nonzero upward trend. In Friedrich et al. (2019), it is additionally shown that all seasonal effects are captured by the model using three Fourier terms. Results for the other series are plotted in Figure 3.5 with a repetition of Figure 3.3 in the top left panel for completeness.

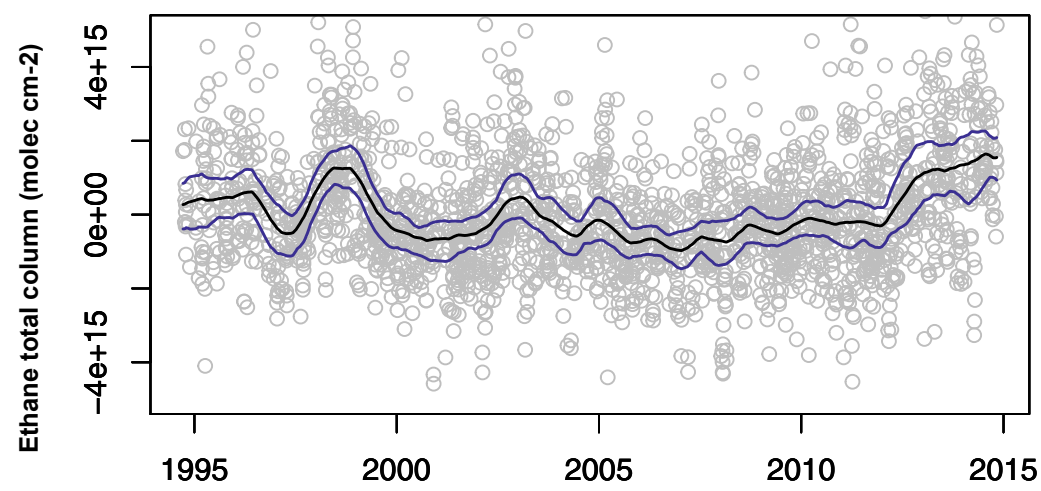

Figure 3.3: Nonparametric trend estimate and confidence bands $(h=0.03)$ 


\subsubsection{Inference on trend shapes}

Based on the trend estimates from previous sections, we are interested in particular features of the trend curve. Having in mind the shape of the trends that we discovered, one important feature for our analysis is the local minimum around $2007 / 2008$ of the trend in the Jungfraujoch ethane column series. Some other ethane series also display a (local) minimum sometime around the period 20082010. Therefore, we are interested in the uncertainty around the location of this minimum. In order to investigate this issue, we propose to use the bootstrap method presented above with an additional step to construct confidence intervals around the minimum location. This is discussed in the first part of this section. The analysis can equally be applied to a local maximum of the trend curve, it is not restricted to the analysis of local minima.

Another interesting feature is the resulting post-minimum upward trend. We have a closer look at the specific form of this trend in the second part. Specifically, we suggest two formal tests; one will compare the nonparametric trend to a linear trend and the other one tests for monotonic behavior in the nonparametric trend. All approaches are applied to investigate the trend in the Jungfraujoch series in the final part of this section.

\section{Analyzing the locations of extrema}

The bootstrap method we use is a simple modification of the bootstrap presented in Section 3.4. Now we are interested in its minimum which we denote by $\hat{g}_{\min }$ and its location by $t_{\text {min }}$. Our goal is to construct a confidence interval for $t_{\text {min }}$. For this new application, we follow Steps 1-4 of Algorithm 3.3 to obtain the bootstrap observations to which we apply the nonparametric estimator. This is the same approach as above, the only new step is that we now determine the location of the local minimum for each bootstrap trend closest to $t_{\text {min }}$ - the original minimum - and denote it by $t_{\text {min }}^{*}$. To be more specific, look at the following bootstrap algorithm:

Algorithm 3.4 (Autoregressive Wild Bootstrap - Minimum location).

1. Repeat steps 1 to 4 of Algorithm 3.3.

2. Determine all local minima of $\hat{g}^{*}(t / n), t=1, \ldots, n$, and select the one closest to $t_{\text {min }}$. Denote the selected position by $t_{\text {min }}^{*}$.

3. Repeat Steps 1 and $2 B$ times to obtain the empirical distribution of $t_{\text {min }}^{*}$. 
In the above algorithm, we need to ensure that we identify the minimum in the bootstrap trend which corresponds to the original global minimum $t_{\min }$. This does not necessarily have to be the global minimum of the bootstrap trend, which could lie far away from the original global minimum. As an empirically satisfactory solution, we therefore use the closest local minimum in Step 2. ${ }^{2}$

The proposed analysis can be used to obtain further evidence on the location of a potential trend reversal and the results could be compared to the break location found in the linear trend analysis discussed in Section 3.3. Compared to the previous analysis, the new approach is less robust in a sense that it is sensitive to the choice of bandwidth that was used to generate the nonparametric trend estimate. It is, however, much more flexible and less restrictive than the breakpoint detection, as we do not force the trend before and after the minimum to be linear. We illustrate this new approach on the ethane data, together with the two tests proposed below, in the final part of this section.

\section{A bootstrap-based test for linearity}

When comparing both approaches, the (piecewise) linear and the nonlinear, an obvious question arises as to whether we can say more about the appropriateness of the two trend shapes. While the linear trend has some desirable properties e.g. we get an estimate of the average annual decrease or increase in the data it might be too restrictive to model the underlying true trend. Kapetanios (2008) designs a bootstrap-based test which can be used to test for parameter constancy under the null hypothesis against smoothly occurring structural change under the alternative. Based on this work, we propose a modification of the test which is able to provide evidence if a certain parametric shape is appropriate to describe the trend in the data at hand. If the true trend follows a certain parametric shape, using an adequate estimator (e.g. the ordinary least squares (OLS) estimator in the linear trend case) is desirable.

For our ethane application, of special interest would be linearity under the null hypothesis. We start by introducing the test in a general framework. The more specific case of linearity will be discussed later. For the general framework, consider the following null hypothesis:

$$
\mathrm{H}_{0}: g(t)=g_{0}(\boldsymbol{\theta}, t) \quad \forall t \in \mathcal{G}_{m},
$$

\footnotetext{
${ }^{2}$ It is worth mentioning that bootstrapping extremes has been proven to be difficult and that the corresponding results should therefore be interpreted with care.
} 
where $g_{0}(\boldsymbol{\theta}, \cdot)$ belongs to a parametric family $\mathbf{G}=\left\{g(\boldsymbol{\theta}, \cdot) ; \boldsymbol{\theta} \in \Theta \subset \mathbb{R}^{d}\right\}$ with $d$ being the number of parameters in $\boldsymbol{\theta}$. Further, the set $\mathcal{G}_{m}=\left\{t_{1}, t_{2}, \ldots, t_{m}\right\}$ contains the time points for which the hypothesis should be tested. Under the alternative, the trend does not follow the parametric shape given by $g_{0}(\tau)$, but can be expressed as in model (3.8). As a special case of the test we can for example consider the linear trend function $g_{0}(t)=\alpha+\beta t$ such that $\boldsymbol{\theta}=(\alpha, \beta)$ and $d=2$. As test statistic in the general case, we use an adaption of the test statistic $\mathcal{T}^{\tau,(\ell)}$ in Kapetanios (2008).

$$
Q^{t}=\left(\hat{g}(t / n)-g_{0}(\widehat{\boldsymbol{\theta}}, t)\right)^{2}
$$

where $\hat{g}(t / n)$ denotes the nonparametric kernel estimator, as before, and $\widehat{\boldsymbol{\theta}}$ denotes the parameter estimates under the null hypothesis. The type of estimator we choose under the null hypothesis depends on the specific case and the form of the parametric function. In the linear trend case, we can use OLS to obtain estimates $\hat{\alpha}$ and $\hat{\beta}$.

As the superscript $t$ shows, this test statistic is pointwise. Since we are interested in the trend over time, we follow Kapetanios (2008) and use the three summary statistics for the set $\mathcal{G}_{m}=\left\{t_{1}, t_{2}, \ldots, t_{m}\right\}$ listed below:

$$
\begin{aligned}
& Q_{\text {ave }}=\frac{1}{m} \sum_{j=1}^{m} \mathcal{T}^{t_{j}} \\
& Q_{\text {sup }}=\sup _{j} \mathcal{T}^{t_{j}} \\
& Q_{\text {exp }}=\frac{1}{m} \sum_{j=1}^{m} e^{\frac{\mathcal{T}^{t_{j}}}{2}}
\end{aligned}
$$

To obtain critical values for the test statistics, we rely again on the same bootstrap method. As Kapetanios (2008) stresses that asymptotic tests show a particularly poor performance in his setting and therefore, we assume that a bootstrap-based test is also preferred in our slightly different scenario. The bootstrap algorithm is presented next. 
Algorithm 3.5 (Autoregressive Wild Bootstrap - Test for a specific trend shape).

1. Estimate $\hat{g}(t / n)$ as in (3.9) for $t=1, \ldots, n$. Obtain the estimate $\widehat{\boldsymbol{\theta}}$ using all data points $t \in \mathcal{G}_{m}$. Then define $\tilde{g}(t)$ as

$$
\tilde{g}(t) \equiv \begin{cases}g_{0}(\widehat{\boldsymbol{\theta}}, t) & \text { for } t \in \mathcal{G}_{m} \\ \hat{g}(t / n) & \text { otherwise }\end{cases}
$$

Obtain a combined residual series $\hat{\epsilon}_{t}=M_{t}\left(\hat{u}_{t}-\tilde{g}(t)\right)$ for $t=1, \ldots, n$.

2. For $0<\gamma<1$, generate $\nu_{1}^{*}, \ldots, \nu_{n}^{*}$ as i.i.d. $\mathcal{N}\left(0,1-\gamma^{2}\right)$ and let $\xi_{t}^{*}=\gamma \xi_{t-1}^{*}+\nu_{t}^{*}$ for $t=2, \ldots, n$. Take $\xi_{1}^{*} \sim \mathcal{N}(0,1)$ to ensure stationarity of $\left\{\xi_{t}^{*}\right\}$.

3. Calculate the bootstrap errors $\epsilon_{t}^{*}$ as $\epsilon_{t}^{*}=M_{t} \xi_{t}^{*} \hat{\epsilon}_{t}$ and generate the bootstrap observations $\left\{\hat{u}_{t}^{*}\right\}_{t=1}^{n}$ by adding the bootstrap errors to the corresponding trend estimate obtained in Step 1:

$$
\hat{u}_{t}^{*}=M_{t}\left(\tilde{g}(t)+\epsilon_{t}^{*}\right)
$$

4. Construct bootstrap versions of the pointwise and summary test statistics and denote them by $Q^{t *}$ and $Q_{i}^{*}$ with $i=$ ave, sup, exp for $t \in \mathcal{G}_{m}$.

5. Repeat Steps 2 to 4 of this algorithm $B$ times to obtain the empirical distribution of $Q_{i}^{*}$ with $i=$ ave, sup, exp and calculate the corresponding critical values and $p$-values from it.

The exact specification of the set $\mathcal{G}_{m}$ highly depends on the application at hand. In practice, often a set of several consecutive points is needed to be able to estimate the parameters under the null hypothesis. This is the case, for example, with the linear trend application that we focus on in the remainder of the section.

\section{Two tests for monotonicity}

In the previous section we proposed a bootstrap-based test to investigate if the trend can be best described by a specific parametric shape - in this case linearity or by the unrestricted nonparametric alternative. In some applications, however, it might not be the most pressing question if the trend follows a specific form. The question whether the trend has been monotonically increasing or decreasing over a certain period can already be enough evidence. In the case of the ethane 
series, geophysicists are mainly interested in establishing an upward trend in the post-minimum period of the sample. Therefore, we propose to additionally use two tests for monotonicity.

For these tests, we consider a situation where the trend function is monotonically increasing under the null hypothesis. The alternative is the same as before, a nonparametric unrestricted trend. Formally, this can be written as:

$\mathrm{H}_{0}: m(\cdot)$ is an increasing function on $\mathcal{I}$,

or, since under the given smoothness assumptions the function $m(\cdot)$ is differentiable:

$$
\mathrm{H}_{0}: m^{\prime}(t / n) \geq 0 \quad \forall t \in \mathcal{I}
$$

In this case, the set $\mathcal{I}$ must be a compact interval in the domain of the function $m(\cdot)$. The paper by Ghosal et al. (2000) proposes the following test statistic to test the above null hypothesis, for $t \in \mathcal{I}$ :

$$
U_{1}^{t}=-\frac{2}{n(n-1)} \sum_{1 \leq i<j \leq n} \operatorname{sign}\left(y_{j}-y_{i}\right) \frac{1}{h_{U}} K\left(\frac{i / n-t / n}{h_{U}}\right) \frac{1}{h_{U}} K\left(\frac{j / n-t / n}{h_{U}}\right)
$$

with

$$
\operatorname{sign}(x)= \begin{cases}1 & \text { if } x>0 \\ 0 & \text { if } x=0 \\ -1 & \text { if } x<0\end{cases}
$$

As kernel function, we use $K(x)=0.75\left(1-x^{2}\right)$ for $-1<x<1$ and 0 otherwise, as Ghosal et al. (2000) suggests. We also follow their bandwidth recommendation $h_{U}=0.5 n^{-1 / 5}$. The test is based on the idea that for an increasing function, increments will be positive and thus, the test statistic should satisfy $U_{1}^{t} \leq 0$ for most $t \in \mathcal{I}$ under the null. This can be easily verified as $U_{t}^{t}$ sums over weighted differences of observations $\left(y_{j}-y_{i}\right)$ such that $j>i$; or more precisely, it sums over the sign thereof. The test statistic $U_{1}^{t}$ corresponds to one point in the interval of interest, $\mathcal{I}$, similar to the test statistic $Q^{t}$ in (3.15). As summary statistic, Ghosal 
et al. (2000) propose a supremum statistic

$$
U_{1, \text { sup }}=\sup _{t \in \mathcal{I}} U_{1}^{t}
$$

Additionally, we use a second test to support our findings. This test is proposed in Chetverikov (2012). The difference compared to (3.19) is the use of the sign function, which is omitted in this version of the test. The full differences and not only their sign will be accounted for. This gives the following test statistic:

$$
U_{2}^{t}=-\frac{2}{n(n-1)} \sum_{1 \leq i<j \leq n}\left(y_{j}-y_{i}\right) \frac{1}{h_{U}} K\left(\frac{i / n-t / n}{h_{U}}\right) \frac{1}{h_{U}} K\left(\frac{j / n-t / n}{h_{U}}\right),
$$

which we apply with the same specifications as we use for $U_{1}^{t}$. Again, this statistic should be negative under the null hypothesis due to the same reason as above. In line with the above procedure, we calculate summary test statistics $U_{2, \text { sup }}$ whose exact definition follow in analogy to $U_{1, \text { sup }}$.

To obtain critical values, we rely once again on the autoregressive wild bootstrap. In this case, we do not need to make many adjustments to the bootstrap given in Algorithm 3.3 for the creation of confidence intervals. Once the bootstrap observations are generated in Step 3, we calculate the test statistics (3.19) and (3.21). Subsequently, we obtain the bootstrap version $U_{1}^{t, *}$ and $U_{2}^{t, *}$ as well as the summary versions. For the linearity testing in the previous section, we needed to estimate a linear trend for part of the sample to be able to conduct the test and thus, an adaption of the bootstrap algorithm was needed, which is not the case here.

\section{Data application}

We now demonstrate the usefulness of our proposed tests by applying it to the Jungfraujoch data. The minimum of interest of the nonparametric trend as seen in Figure 3.3 is located at 2007.04. When applying the bootstrap tool to obtain $95 \%$ confidence intervals, we find them to range from 2006.30 to 2007.68, which is located slightly earlier than the confidence interval of the break location in the parametric setting (2008.35 to 2010.03). Although both results are constructed using substantially different approaches, the intervals lie close together. The nonparametric approach results in a smooth trend, while the parametric specification includes an abrupt break through which the minimum is defined. Similar results 
can be obtained for the Toronto ethane series with a minimum in 2008.84 and the 95\% confidence interval ranging from 2007.30 to 2010.36.

Of special interest in our ethane series analysis is the post minimum development of the trend. Multiple series in the Northern Hemisphere display an upward trend after 2007/2008 and in previous research this trend has been modeled by a linear trend. Our approach goes back one step and first investigates the question whether a linear trend or a non-linear trend as in the nonparametric case is preferred. To this end, we apply the above procedure with a linear trend specification under the null to the Jungfraujoch data. For this we select the set $\mathcal{G}_{m}$ in such a way that it covers the period after 2007.04 where the minimum is obtained.

As mentioned above, in the case of a linear trend under the null hypothesis, we need the set to consist of a series of consecutive points to be able to estimate the slope parameter. This is done here by taking approximately the last quarter of the sample. If it was exactly the last quarter, the set would look like $\mathcal{G}_{m}=$ $\left\{\frac{3}{4} n, \frac{3}{4} n+1, \ldots, n\right\}$, which satisfies the theoretical requirements. Under the null, the trend curve is designed in such a way that the linear trend starts at the point where the nonparametric trend ends. Thus, it starts at the point $\hat{g}_{\min }$. Therefore, the intercept is fixed and only the slope parameter is estimated under the null hypothesis by OLS. For the calculation of the test statistic (3.15) we use as $g_{0}(\widehat{\boldsymbol{\theta}}, t)$ the best fitting linear trend line that goes through the minimum for all $t$ in $\mathcal{G}_{m}$.

An application of this procedure to the Jungfraujoch ethane series gives us three $p$-values of $p_{\text {ave }}=0.002, p_{\text {sup }}=0.004$ and $p_{\exp }=0.000$. We note that for the exponential summary statistic to work, we first need to downscale the series by $10^{13}$. This does not change the shape of the trend, it just makes the test feasible. All three tests unanimously reject the null hypothesis of a linear trend for the last period of the sample.

Coming back to the original research question and motivation for this test, we now investigate the post-minimum nonparametric trend of the Jungfraujoch ethane series. After having rejected linearity, this test helps us to establish whether there has been a monotonic upward trend in the series since the minimum at the beginning of 2007. This defines the way we choose the interval $\mathcal{I}$ in this application over which we test for monotonicity - the starting point is the minimum at 2007.04 and the end point is the end of the sample at 2014.83. The interval thus defined contains 930 out of the 2260 time points. It coincides with the set of points we chose for the linearity test above. 
After computing the two different versions $U_{1}^{t}$ and $U_{2}^{t}$ with $h_{U}=0.107$ for all points in the interval, we obtain $U_{1, \text { sup }}=-0.008$ and $U_{2, \text { sup }}=-4.061 \times 10^{13}$. The bootstrap critical values for the tests are 0.0269 and $1.176 \times 10^{14}$, respectively, which yield $p$-values of 0.371 for $U_{1, \text { sup }}$ and 0.863 for $U_{2, \text { sup }}$. Therefore, we cannot reject the null hypothesis and conclude that the post-minimum trend in the ethane burden on top of the Jungfraujoch is likely to be monotonically increasing.

\subsection{Conclusion}

In this chapter, two approaches are proposed to estimate trends in climate time series: a broken linear trend model with unknown break date and a nonlinear trend model, which is estimated nonparametrically. Both methods are applied to a time series of ethane total columns measured at a station in the Swiss Alps. The high-altitude station located at the Jungfraujoch records daily observations of ethane abundance in the atmosphere. Depending on the conditions, however, measurements cannot be made every day resulting in a daily time series with approximately $70 \%$ missing observations. This is a limitation frequently encountered in (climatological) time series. It causes a problem when we want to construct confidence intervals around the trend estimate. An additional complication arises due to strong seasonal effects, which cause the residuals from both models to be serially correlated. In order to resolve these issues, we suggest an autoregressive wild bootstrap method for the construction of confidence intervals around the break location and the parameter estimates of the parametric model. For the nonparametric model, we propose a battery of diagnostic tools to investigate the shape of the resulting trend.

Results from this application indicate that there is a significant upward trend in atmospheric ethane, starting around $2007 / 2008$. This is confirmed by both approaches, the break of the linear model is located at the beginning of 2009, while the local minimum of the nonparametric approach is in 2007. Additionally, after 2007 we cannot embed a straight line into the simultaneous confidence bands around the nonparametric trend estimate which is supported by the subsequent results of a formal test for linearity. All three proposed test statistics indicate that a linear trend is not appropriate for the post-minimum period of the Jungfraujoch data. In addition, the nonparametric estimation reveals a trend function which exhibits a local maximum around the years of 1998 and 2002-2003 which coincide with boreal forest fires in Russia. 
Given both sets of results, it should be stressed that the two approaches proposed in this chapter can be seen as complimentary rather than competing methods. The simplicity of the broken linear trend model allows us to indicate a numerical value for the slope parameter, summarizing the development of the trend over a particular period. The complexity of the nonlinear approach has the potential of providing us with additional information, while at the same time it can be used to confirm previous findings.

One, perhaps seemingly unnecessary, limitation of the broken linear trend model, is that it can accommodate only one break, putting it at a natural disadvantage to the more flexible nonparametric approach. Indeed, estimation of broken linear trend models with multiple breaks at unknown locations can be estimated using, for instance, the methods proposed in Bai and Perron (1998), which also allow one to test for the number of breaks in the trend. Constructing confidence intervals for the locations of the breaks is, however, more complicated in such models. It can be a powerful tool for the analysis of more complicated time series than atmospheric ethane, whose properties are less well studied. The extension of the bootstrap methodology to multiple breaks is left for future research.

\section{A Additional results}

In this first part of the Appendix we give additional results for the complete set of ethane series. Table 3.3 shows results of bandwidth selection for the nonparametric part of the analysis. They are obtained by setting $k=5$ in 3.10 , meaning 11 observations are left out in total. Using $k=4$ produced almost identical results. Figures 3.4 and 3.5 display the results of the broken linear trend and the nonparametric trend, respectively. The latter figure is produced using the first column of bandwidths in Table 3.3 .

\begin{tabular}{lcc}
\hline & First & Second \\
\cline { 2 - 3 } Jungfraujoch & 0.030 & 0.065 \\
Boulder & 0.060 & 0.220 \\
Eureka & 0.080 & 0.080 \\
Lauder & 0.110 & 0.220 \\
Thule & 0.060 & 0.120 \\
Toronto & 0.085 & 0.130 \\
\hline
\end{tabular}

Table 3.3: $\overline{\text { Bandwidth selection using MCV }}$ with $k=5$ 


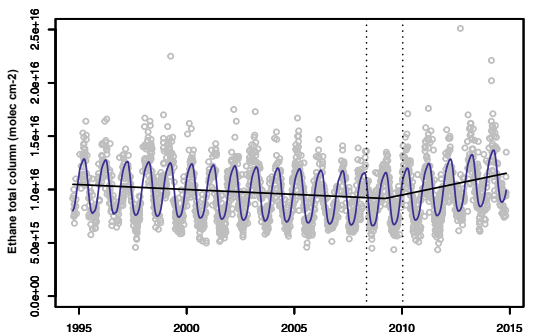

(a) Jungfraujoch

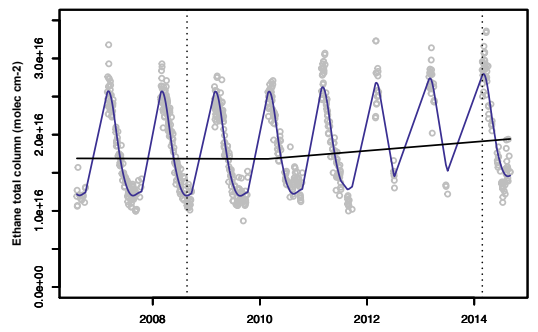

(c) Eureka

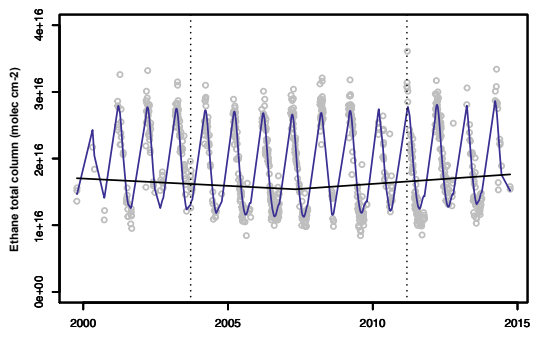

(e) Thule

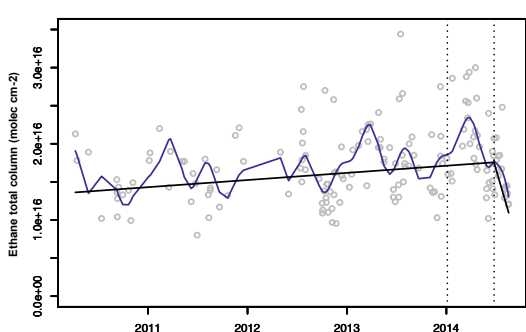

(b) Boulder

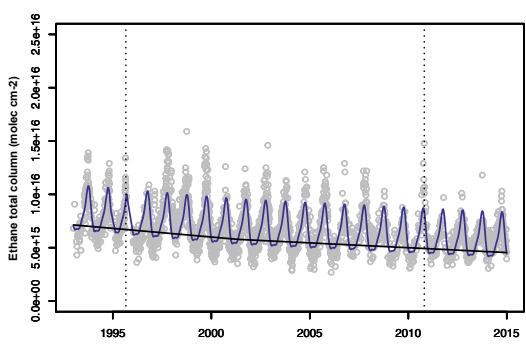

(d) Lauder

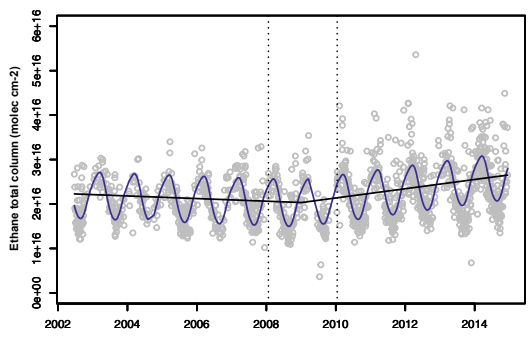

(f) Toronto

Figure 3.4: This figure shows the data as well as the continuous broken trend and the fitted Fourier series for all 6 series. 


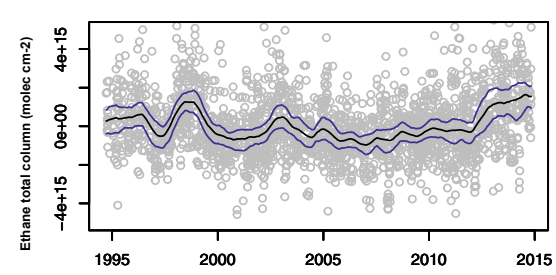

(a) Jungfraujoch

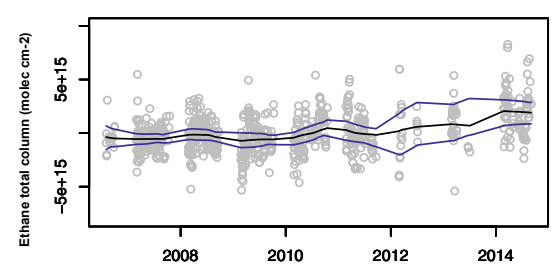

(c) Eureka

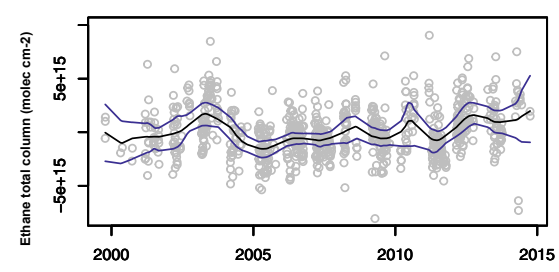

(e) Thule

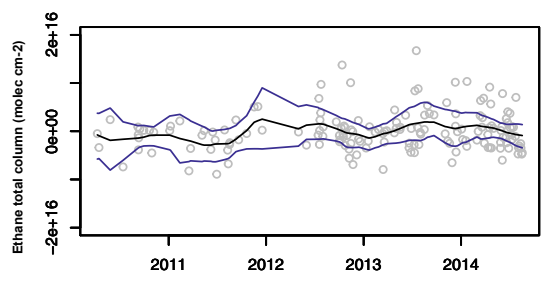

(b) Boulder

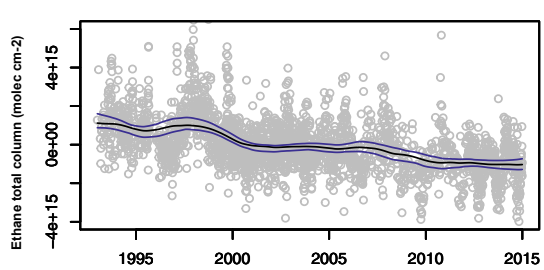

(d) Lauder

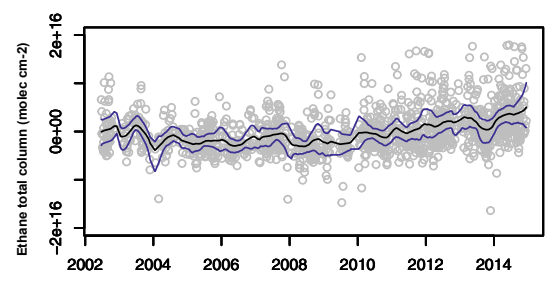

(f) Toronto

Figure 3.5: This figure shows the data, the nonparametric trend functions and the $95 \%$ simultaneous confidence bands. 


\section{B Simulation study}

To support the choice of the method proposed in Algorithm 3.4, we conduct a small Monte Carlo experiment to investigate the coverage and the width of the resulting confidence intervals in finite samples. In order to do so, we select a simple DGP with a trend function that contains two minima. The trend is given by a smooth transition model with four transitions and the following specifications:

$$
y_{t}=\delta(t)+u_{t}
$$

with $\delta(t)$ given by

$$
\begin{aligned}
\delta(t)= & \beta_{1} t / n+\beta_{2} t / n G\left(t / n, \lambda, c_{1}\right)+\beta_{1} t / n G\left(t / n, \lambda, c_{2}\right) \\
& +\beta_{2} t / n G\left(t / n, \lambda, c_{3}\right)+\beta_{1} t / n G\left(t / n, \lambda, c_{4}\right) .
\end{aligned}
$$

The transition function is $G(t, \lambda, c)=(1+\exp \{-\lambda(t-c)\})^{-1}$, where $\lambda$ determines the smoothness of the transition and $c$ specifies its location.

The errors $\left\{u_{t}\right\}$ follow an $\operatorname{AR}(1)$ process

$$
u_{t}=\phi u_{t-1}+\epsilon_{t} \quad \text { with } \quad \epsilon_{t} \sim N\left(0, \frac{1}{9}\left(1-\phi^{2}\right)\right) \text {. }
$$

We can thus vary the degree of dependence present in this study by choosing different values for the AR parameter $\phi$. Regarding the shape of the trend function given by (3.22), we fix $c_{1}=0.1, c_{2}=0.4, c_{3}=0.6$ and $c_{4}=0.9$ as well as $\beta_{1}=1$ and $\beta_{2}=-1$. We can vary how pronounced the two minima are by selecting different values for the parameter $\lambda$, here we consider three different values $\lambda=7,8,9,10$. Two examples are displayed in Figure 3.6. Panel (a) plots the trend function when $\lambda=10$. The two minima are clearly distinguishable from one another in this specification. In Panel (b) we set $\lambda=7$ which makes the minima less pronounced. We are interested in the second minimum and use our proposed procedure to construct confidence intervals around its location with a nominal coverage of $95 \%$. We look at two different bandwidths and three different sample sizes. For each specifications, we generate 1000 samples from the DGP. In the bootstrap, we set $C=0.5$ and $B=1999$.

Table 3.4 displays the results for a sample size of 100. The numbers represent the coverage probabilities of the generated confidence intervals. They count how often the true minimum fall within the confidence intervals. The numbers in brack- 


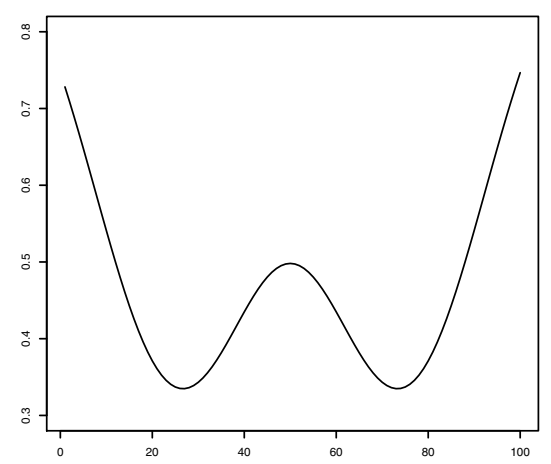

(a) $\lambda=10$

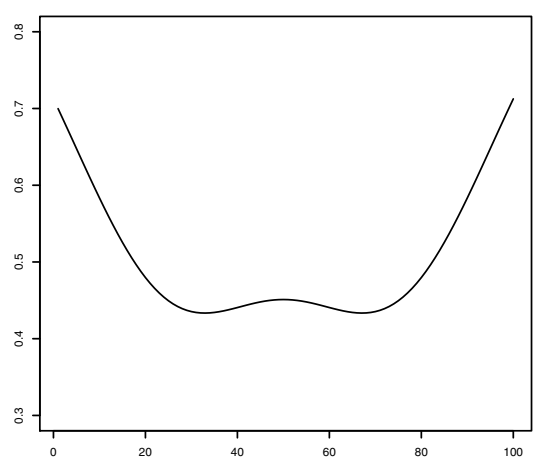

(b) $\lambda=7$

Figure 3.6: Visualizing the trend function as given by $(3.22)$

ets underneath the coverage probabilities are the corresponding average interval lengths. The different rows look at different values of $\lambda$ while the columns change the correlation coefficient $\phi$. As a general pattern it emerges that the confidence intervals become wider the higher the degree of serial correlation. The reverse holds true for the lengths of the intervals and the values of $\lambda$ : the higher $\lambda$, the more narrow the intervals get. This is natural as the minima get more pronounced the larger the value of $\lambda$.

The coverage is too low in three cases considered in Table 3.4. For the two small values of $\lambda$ and no serial correlation, the coverage probability is $56 \%$ and

\begin{tabular}{ccccc}
\hline$\phi=$ & 0 & 0.1 & 0.3 & 0.5 \\
\hline$\lambda=7$ & 0.56 & 0.90 & 0.96 & 0.99 \\
& $(14.10)$ & $(14.06)$ & $(14.63)$ & $(15.37)$ \\
$\lambda=8$ & 0.75 & 0.97 & 1.00 & 1.00 \\
& $(13.09)$ & $(13.12)$ & $(13.44)$ & $(14.21)$ \\
$\lambda=9$ & 0.95 & 1.00 & 1.00 & 1.00 \\
& $(11.46)$ & $(12.02)$ & $(12.17)$ & $(12.15)$ \\
$\lambda=10$ & 1.00 & 1.00 & 1.00 & 1.00 \\
& $(10.12)$ & $(10.48)$ & $(10.90)$ & $(10.60)$ \\
\hline
\end{tabular}

Table 3.4: Coverage probabilities and widths of confidence intervals for $h=0.04$ and $n=100$ 
$75 \%$. For $\lambda=7$ and $\phi=0.1$, the intervals cover the true minimum $90 \%$ of the times. In all other cases, the coverage lies close to or above the nominal level of $95 \%$. The dependence on $\lambda$ is not surprising since it is easier for the nonparametric estimator to recover the true minimum when it is more pronounced. In this case, the minima of the estimated bootstrap trends lie closer together and closer to the true minimum, leading to a narrower interval and higher coverage.

Table 3.5 shows results for a larger bandwidth $(h=0.08$ compared to $h=0.04$ before). In addition, the three different parts investigate an increasing sample size. In the upper part, we let $n=100$ as in Table 3.4. In the middle part the sample size increases to 500 and in the bottom part to 700. The rest of the table is organized in the same way as Table 3.4, rows vary $\lambda$ and columns present different values of $\phi$. For the larger sample sizes we only consider two different specifications of $\lambda: \lambda=7$ and $\lambda=9$.

Increasing the bandwidth leads to an improvement in coverage in the cases that previously resulted in low coverage. We observe that the case with no autocorrelation and $\lambda=7$ remains problematic even when we increase the sample size. All other cases produce accurate or too high coverage probabilities. This leads us to conclude that the method shows good performance in this short simulation experiment as long as the local extremum under consideration is pronounced enough. When it is not very pronounced a larger bandwidth seems preferable. 


\begin{tabular}{|c|c|c|c|c|}
\hline \multicolumn{5}{|c|}{$n=100$} \\
\hline$\phi=$ & 0 & 0.1 & 0.3 & 0.5 \\
\hline$\lambda=7$ & $\begin{array}{c}0.71 \\
(14.58)\end{array}$ & $\begin{array}{c}0.90 \\
(13.96)\end{array}$ & $\begin{array}{c}0.96 \\
(14.53)\end{array}$ & $\begin{array}{c}0.99 \\
(14.88)\end{array}$ \\
\hline$\lambda=8$ & $\begin{array}{c}0.82 \\
(12.85)\end{array}$ & $\begin{array}{c}0.93 \\
(12.84)\end{array}$ & $\begin{array}{c}0.98 \\
(13.72)\end{array}$ & $\begin{array}{c}1.00 \\
(14.00)\end{array}$ \\
\hline$\lambda=9$ & $\begin{array}{c}0.92 \\
(11.42)\end{array}$ & $\begin{array}{c}0.99 \\
(11.30)\end{array}$ & $\begin{array}{c}1.00 \\
(12.49)\end{array}$ & $\begin{array}{c}1.00 \\
(12.47)\end{array}$ \\
\hline$\lambda=10$ & $\begin{array}{c}0.99 \\
(10.26)\end{array}$ & $\begin{array}{c}1.00 \\
(10.46)\end{array}$ & $\begin{array}{c}1.00 \\
(10.83)\end{array}$ & $\begin{array}{c}0.99 \\
(11.04)\end{array}$ \\
\hline \multicolumn{5}{|c|}{$n=500$} \\
\hline & $\begin{array}{c}0.67 \\
(60.72)\end{array}$ & $\begin{array}{c}0.90 \\
(59.01)\end{array}$ & $\begin{array}{c}0.97 \\
(61.96)\end{array}$ & $\begin{array}{c}0.98 \\
(62.57)\end{array}$ \\
\hline$\lambda=9$ & $\begin{array}{c}0.95 \\
(33.88)\end{array}$ & $\begin{array}{c}1.00 \\
(35.44)\end{array}$ & $\begin{array}{c}1.00 \\
(39.34)\end{array}$ & $\begin{array}{c}1.00 \\
(40.99)\end{array}$ \\
\hline \multicolumn{5}{|c|}{$n=700$} \\
\hline$\lambda=7$ & $\begin{array}{c}0.80 \\
(78.74)\end{array}$ & $\begin{array}{c}0.95 \\
(79.22)\end{array}$ & $\begin{array}{c}0.98 \\
(82.03)\end{array}$ & $\begin{array}{c}1.00 \\
(82.96)\end{array}$ \\
\hline$\lambda=$ & $\begin{array}{c}0.96 \\
(40.49)\end{array}$ & $\begin{array}{c}1.00 \\
(43.30)\end{array}$ & $\begin{array}{c}1.00 \\
(45.91)\end{array}$ & $\begin{array}{c}1.00 \\
(49.49)\end{array}$ \\
\hline
\end{tabular}

Table 3.5: Coverage probabilities and widths of confidence intervals for $h=0.08$ and an increasing sample size $n$. 



\section{Chapter 4}

\section{Bootstrap Simultaneous}

\section{Confidence Bands For}

\section{Time-varying Coefficient}

\section{Models}

This chapter investigates bootstrap methods to construct simultaneous confidence bands for time-varying coefficient regression models. For this purpose an existing sieve bootstrap method for deterministic trends is extended to time-varying coefficient models. The sieve bootstrap relies on i.i.d. resampling which is applied to the residuals of an autoregressive fit. In the proposed case, the sieve is based on nonparametric local linear estimation of the coefficient curves. An extensive simulation study looking at two different data generating processes and various degrees of autocorrelation and heteroskedasticity investigates the small sample properties of the proposed bootstrap methods. We show that the methods perform well in finite samples. An empirical application to assess the price development of $\mathrm{CO} 2$ certificates in the European Emissions Trading Scheme (EU ETS) demonstrates the relevance of the methods in practice. 



\subsection{Introduction}

The economy is constantly evolving and so are economic variables and the relation between them. There is little doubt that structural change is present. The ways in which these changes can occur are, however, of diverse nature and this constitutes a major challenge in econometric modeling. Although both empirical and theoretical research suggests that structural changes require an adapted modeling strategy, traditional parametric models with constant coefficients are often used as a standard tool when estimating the relation between economic variables.

The fact that many economic relations experience fundamental changes calls for more flexible econometric models such as time-varying coefficient models. These models can be formalized both parametrically and nonparametrically. Nonparametric models have the advantage of flexibility since no prior information on the functional form of the coefficient curves is needed. Researchers might not know the form of the change and therefore, such models allow them to obtain a first impression and they can be used as an initial step in modeling. Due to their flexibility and robustness to functional form misspecification, they are very appealing to use in a situation where smooth structural change is suspected.

Early work on the topic of time-varying coefficient models is by Robinson (1989) who establishes the idea in a linear regression setup. An extension of his work is given in Orbe et al. (2005) and Orbe et al. (2006). A more general model of this kind is presented in Cai (2007) and more recent work in this field includes Kapetanios (2008), Liang and Li (2012) and Kristensen (2012).

Estimating trends or parameter curves is an important first step to learn about the form of the investigated relation. It is, nevertheless, crucial to be able to assess the uncertainty around the estimates. Cai (2007) derives confidence intervals for his nonparametric estimator with the help of asymptotic theory. These confidence intervals are, however, only valid for one point in time on the estimated curve. More informative in the context of assessing changing economic relations and trends are confidence bands, which are valid over more than one point in time. Many interesting research questions, like whether a coefficient remains zero or experiences an upward trend over a certain period of time, cannot be answered with pointwise confidence intervals. The concept of simultaneous confidence bands should be used to answer these questions, but in the context of nonparametric estimation, they are difficult to derive with the help of asymptotic theory. Nevertheless, asymptotic theory has been successfully used to construct simultaneous 
confidence bands for nonparametric, time-varying coefficient models. For example, Wu and Zhao (2007) develop simultaneous confidence bands in the case of smooth trends. For the more general model by Cai (2007), it is Zhou and Wu (2010), who provide simultaneous confidence bands with asymptotically correct nominal coverage probabilities.

An alternative to asymptotic theory are bootstrap methods. Compared to inference based on asymptotic theory, bootstrap methods are more robust to misspecification and have a better overall small sample performance. As the bootstrap was originally designed to deal with data without serial dependence, it needs to be modified in a time series set-up. There are several modifications of the classical bootstrap that are able to handle heteroskedasticity as well as serial correlation. A wild bootstrap approach, originally proposed by $\mathrm{Wu}$ (1986), is designed for the context of heteroskedasticity. Both a block bootstrap, in its most famous form dating back to Künsch (1989), and a sieve bootstrap invented by Kreiss (1992) and Bühlmann (1997) have been developed for serially correlated time series data. For nonparametric trend estimation, a sieve bootstrap method is proposed by Bühlmann (1998). Further research on bootstrap methods for nonparametric trend estimation is presented in Neumann and Polzehl (1998), who use a wild bootstrap method to obtain simultaneous confidence bands around a trend function.

The purpose of this chapter is to present and investigate augmented sieve bootstrap methods, which are able to provide reliable inference on time-varying coefficient models. In particular, the bootstrap methods can be used to obtain simultaneous confidence bands around estimated parameter curves. In this context, bootstrap methods have, to our knowledge, not been proposed in the literature. It is important to investigate the performance of the method in detail before applying it in practice. This is why we conduct an extensive Monte Carlo simulation with many different parameter specifications. This helps us understand where the strengths and weaknesses of the methods lie before applying it to real data in our empirical example.

We investigate the relationship between the prices of CO2 emission allowances and their fundamental price drivers in the European Emissions Trading Scheme (EU ETS). The empirical application underlines the relevance and importance of time-varying coefficient models and, in particular, flexible bootstrap tools in practice. 
Finally, a word on notation. Bootstrap quantities are given a subscript *, expressing that it is conditional on the original sample. Additionally, for any function $f(x), f^{(i)}(x)=\frac{d^{i}}{d x^{i}} f(x)$ denotes the $i$-th derivative with respect to $x$.

\subsection{The model}

Consider the following linear time-varying coefficient model, for $t=1, \ldots, n$,

$$
y_{t}=\boldsymbol{\beta}_{t}^{\prime} \mathbf{x}_{t}+z_{t}=\beta_{0, t}+\sum_{j=1}^{d} \beta_{j, t} x_{j, t}+z_{t},
$$

where $\boldsymbol{\beta}_{t}=\left(\beta_{0, t}, \beta_{1, t}, \ldots, \beta_{d, t}\right)^{\prime}$ and $\mathbf{x}_{t}=\left(x_{0, t}, x_{1, t}, \ldots, x_{d, t}\right)^{\prime}$ represent the $(d+1)$ dimensional time-varying coefficients and covariate process, respectively. Further, we have that $z_{t}=\sigma_{t} u_{t}$ with $\sigma_{t}$ capturing potential heteroskedasticity and $\left\{u_{t}\right\}$ being a linear process:

$$
u_{t}=\sum_{j=0}^{\infty} \psi_{j} \epsilon_{t-j} \quad \text { with } \quad \psi_{0}=1,
$$

with autocovariance function $R_{U}(k)=\mathbb{E} u_{t} u_{t+k}$ and long-run variance

$$
\Omega_{U}=\sum_{k=-\infty}^{\infty} \mathbb{E} u_{t} u_{t+k}=\sum_{k=-\infty}^{\infty} R_{U}(k),
$$

where $\left\{\epsilon_{t}\right\}$ is an i.i.d. series with $E\left(\epsilon_{t}\right)=0$ and $\mathbb{E}\left(\epsilon_{t}\right)^{2}=\sigma_{\epsilon}^{2}=\left(\sum_{j=0}^{\infty} \psi_{j}^{2}\right)^{-1}$, and $\mathbb{E}\left(\epsilon_{t}^{4}\right)<\infty$. With this specification, we allow for both autocorrelation and heteroskedasticity in the error term of our model.

The focus of this chapter lies on the nonparametric estimation of and inference on the parameter curves $\beta_{j, t}=\beta_{j}(\cdot)$, for $j=0, \ldots, d$. They have to be sufficiently smooth for the estimation method to work. More specifically, this means they have to be twice continuously differentiable on $(0,1)$ with $\sup _{0<\tau<1}\left|\beta_{j}^{(i)}(\tau)\right|<\infty$, for $i=0,1,2$. As first proposed in Robinson (1989), the $\beta_{j}(\cdot)$ 's are dependent on the sample size $n$ through the so-called intensity assumption. For every $j=$ $0, \ldots, d$, they are functions on the unit interval: $\beta_{j}:[0,1] \rightarrow \mathbb{R}$. Intuitively, this assumption expresses the need to increase the amount of local information on which the estimator depends and it is crucial to obtain consistency. In other words, it means that the support of the estimators needs to become more dense 
as $n$ increases, which would not be the case in this framework, if this assumption did not exist, since the support is the set of natural numbers (Robinson, 1989).

The model was first introduced by Robinson (1989), who assumed independent errors and who did not allow for heteroskedasticity. Further extensions are given in Robinson (1991) as well as in Orbe et al. (2005) and Orbe et al. (2006). These papers have in common that they use the well-known nonparametric method of Nadaraya-Watson to obtain estimated coefficient curves. While this method is intuitive and frequently used, the disadvantage of this local constant estimation method is that it is known to suffer from boundary effects and has a larger bias than local linear or local polynomial approaches (Fan and Gijbels, 1996). In response to this, Cai (2007) proposes a local linear estimator of model (4.1) and compares it to the Nadaraya-Watson estimator in this setting. His findings support the general results of Fan and Gijbels (1996): it is beneficial to use a local linear approach for this model. This is why we focus on this approach here.

\subsection{Nonparametric estimation}

In this section, we first present the details of the nonparametric estimator. As mentioned in the previous section, we use the local linear kernel smoother as was first proposed for this model by Cai (2007). In this approach, the bandwidth parameter plays a crucial role such that we devote a second part of this section to discussing methods for bandwidth selection.

\subsubsection{The local linear estimator}

Given an observed set of data on the response series $\left\{y_{t}\right\}$ and the regressors $\left\{\mathbf{x}_{t}\right\}$, the coefficient curves $\beta_{j}(\cdot)$ can be estimated via local linear nonparametric kernel estimation. Underlying this method is a first-order Taylor approximation of each $\beta_{j}(\cdot)$, for $j=0, \ldots, d$, at a fixed time point $\tau \in(0,1)$. For $t / n$ in a neighborhood of $\tau$ it holds that

$$
\beta_{j}(t / n) \approx \beta_{j}(\tau)+\beta_{j}^{(1)}(\tau)(t / n-\tau)
$$

with $\beta_{j}^{(1)}(\cdot)$ denoting the first derivative of the coefficient function $\beta_{j}(\cdot)$. If we replace $\beta_{j, t}=\beta_{j}(t / n)$ by approximation (4.3) for every $j=0, \ldots, d$, equation (4.1) 
can now be rewritten as:

$$
y_{t} \approx \boldsymbol{\beta}(\tau)^{\prime} \mathbf{x}_{t}+\boldsymbol{\beta}^{(1)}(\tau)^{\prime} \mathbf{x}_{t}(t / n-\tau)+z_{t}
$$

where $\boldsymbol{\beta}^{(1)}(\tau)=\left(\beta_{0}^{(1)}(\tau), \cdots, \beta_{d}^{(1)}(\tau)\right)^{\prime}$ denote the stacked first derivatives of trend functions evaluated at $\tau$. The local linear estimator of this model is found by minimizing the following weighted sum of squares with respect to $\boldsymbol{\theta}$, where $\tilde{\mathbf{x}}_{t}(\tau)=\left(\mathbf{x}_{t}, \mathbf{x}_{t}(t / n-\tau)\right)^{\prime}:$

$$
\widehat{\boldsymbol{\theta}}(\tau)=\operatorname{argmin}_{\boldsymbol{\theta}} \sum_{s=1}^{n}\left\{y_{s}-\tilde{\mathbf{x}}_{s}(\tau)^{\prime} \boldsymbol{\theta}\right\}^{2} K\left(\frac{s / n-\tau}{h}\right),
$$

where $K(\cdot)$ is a kernel function and $h>0$ is a bandwidth. As $n \rightarrow \infty$, the bandwidth is assumed to satisfy $h \rightarrow 0$ while $n h \rightarrow \infty$. The solution to this minimization problem gives the estimator of $(d+1)$ coefficient functions $\boldsymbol{\beta}(\cdot)$ as well as their $(d+1)$ first derivatives $\boldsymbol{\beta}^{(1)}(\cdot)$. Let $\boldsymbol{\theta}(\tau)=\left(\boldsymbol{\beta}(\tau), \boldsymbol{\beta}^{(1)}(\tau)\right)^{\prime}$ denote the vector of stacked coefficient functions and first derivatives. Then, the estimator $\widehat{\boldsymbol{\theta}}(\tau)=\left(\widehat{\boldsymbol{\beta}}(\tau), \widehat{\boldsymbol{\beta}}^{(1)}(\tau)\right)^{\prime}$ can be expressed as,

$$
\widehat{\boldsymbol{\theta}}(\tau)=\left(\begin{array}{cc}
\mathbf{S}_{n, 0}(\tau) & \mathbf{S}_{n, 1}^{\prime}(\tau) \\
\mathbf{S}_{n, 1}(\tau) & \mathbf{S}_{n, 2}(\tau)
\end{array}\right)^{-1}\left(\begin{array}{c}
\mathbf{T}_{n, 0}(\tau) \\
\mathbf{T}_{n, 1}(\tau)
\end{array}\right) \equiv \mathbf{S}_{n}^{-1}(\tau) \mathbf{T}_{n}(\tau)
$$

for $\tau \in(0,1)$, where for $k=0,1,2$,

$$
\begin{aligned}
& \mathbf{S}_{n, k}(\tau)=\frac{1}{n h} \sum_{t=1}^{n} \mathbf{x}_{t} \mathbf{x}_{t}^{\prime}(t / n-\tau)^{k} K\left(\frac{t / n-\tau}{h}\right), \\
& \mathbf{T}_{n, k}(\tau)=\frac{1}{n h} \sum_{t=1}^{n} \mathbf{x}_{t}(t / n-\tau)^{k} K\left(\frac{t / n-\tau}{h}\right) y_{t} .
\end{aligned}
$$

This estimator can be seen as a weighted least squares estimator of a model of the form (4.4). The fitted values are thus obtained by

$$
\hat{y}_{t}=\mathbf{x}_{t}^{\prime} \widehat{\boldsymbol{\beta}}(t / n)
$$

The kernel function has to satisfy certain assumptions: $K(\cdot)$ is a probability density, symmetric, twice continuously differentiable and with compact support. 


\subsubsection{Bandwidth selection}

A crucial aspect of any local fitting method is the choice of the bandwidth parameter $h$. A large value of this parameter will lead to an estimated curve which is very smooth while a small value will result in a wiggly estimate. A theoretically optimal bandwidth can be obtained but it is infeasible for practical use as the expression depends on several unobservable quantities, e.g. the second derivative of $\boldsymbol{\beta}(\cdot)$. We refer the interested reader to Fan and Gijbels (1996) for more details. We focus on rules how to select bandwidths in practice.

Many data-driven methods for bandwidth selection are based on the principle of cross validation (CV). The basic idea is to find the value of the bandwidth that provides the best fit in terms of minimizing the sum of squared residuals without overfitting. Simply finding $h$ that minimizes the sum of squared residuals creates a problem of overfitting, since for $h \rightarrow \infty$ the estimate approaches the data points. In the limiting case, the estimated parameter curve would be equal to linear interpolation of the observations. This obviously problematic procedure would select the smallest considered bandwidth in all cases. The leave-one-out estimator provides a way to circumvent overfitting. The first step is to construct the leave-one-out estimator for every time point by leaving out the observation $t$ that receives the highest weight in the local estimation. This leave-one-out estimator is denoted by $\hat{\boldsymbol{\beta}}_{h,-t}(\cdot)$. The second step in the least-squares CV approach is to look at the weighted average of the leave-one-out squared residuals

$$
C V(h)=\frac{1}{n} \sum_{t=1}^{n}\left\{y_{t}-\mathbf{x}_{t}^{\prime} \hat{\boldsymbol{\beta}}_{h,-t}(t / n)\right\}^{2}
$$

and minimize this criterion with respect to $h$.

Cross validation, however, was originally designed for independent data and can therefore cause problems in time series applications. Chu and Marron (1991) show that cross validation systematically chooses bandwidths that are too small (too large) in the presence of positive (negative) autocorrelation. They propose a modification of the criterion called modified cross validation (MCV) and show that it works well in time series applications. It follows the same general principle as CV, but it is based on a different estimator. Chu and Marron (1991) use a leave- $(2 l+1)$-out version of the leave-one-out estimator. The estimator as given in equation (4.6) will be subject to minor changes such that $\mathbf{S}_{n, k}(\tau)$ and $\mathbf{T}_{n, k}(\tau)$ 
are replaced by the following leave- $(2 l+1)$-out counterparts

$$
\begin{aligned}
& \mathbf{S}_{n, k}^{l, h}(\tau)=\frac{1}{(n-2 l-1) h} \sum_{t:|t-\tau n|>l} \mathbf{x}_{t} \mathbf{x}_{t}^{\prime}(t / n-\tau)^{k} K\left(\frac{t / n-\tau}{h}\right), \\
& \mathbf{T}_{n, k}^{l, h}(\tau)=\frac{1}{(n-2 l-1) h} \sum_{t:|t-\tau n|>l} \mathbf{x}_{t}(t / n-\tau)^{k} K\left(\frac{t / n-\tau}{h}\right) y_{t},
\end{aligned}
$$

for $k=0,1,2$. For $l=0$, the original cross validation leave-one-out estimator is obtained.

In addition to cross validation and modified cross validation, we follow the suggestions in Cai (2007) as well as Zhou and Wu (2010) and investigate the performance of two additional bandwidth selection methods in our simulation study and application. Cai (2007) considers an approach based on the Akaike information criterion (AIC) while Zhou and $\mathrm{Wu}$ (2010) use generalized cross validation (GCV) originally proposed by Craven and Wahba (1978).

Both approaches use the $n$ by $n$ matrix $Q_{h}$, sometimes called hat-matrix, with which we can multiply $\left\{y_{t}\right\}$ to obtain the fitted values $\left\{\hat{y}_{t}\right\}$. Formally, we can write $\hat{\mathbf{y}}=Q_{h} \mathbf{y}$, where $\mathbf{y}=\left(y_{1}, \ldots, y_{n}\right)^{\prime}$ and $\hat{\mathbf{y}}=\left(\hat{y}_{1}, \ldots, \hat{y}_{n}\right)^{\prime} .{ }^{1}$ Then, the GCV and AIC criterion are defined as

$$
\begin{aligned}
G C V(h) & =\frac{n^{-1}|\mathbf{y}-\hat{\mathbf{y}}|^{2}}{\left(1-\operatorname{tr}\left\{Q_{h}\right\} / n\right)^{2}}, \\
A I C(h) & =\log \hat{\sigma}^{2}+2 \frac{\operatorname{tr}\left\{Q_{h}\right\}+1}{n-\operatorname{tr}\left\{Q_{h}\right\}-2},
\end{aligned}
$$

where $\hat{\sigma}^{2}=(1 / n) \sum_{t=1}^{n}\left(y_{t}-\hat{y}_{t}\right)^{2}$.

\subsection{Confidence intervals with the sieve (wild) bootstrap}

To obtain confidence intervals we propose two new bootstrap methods which are an extension of the existing sieve bootstrap method developed by Bühlmann (1998), originally designed for a deterministic trend model. The first method is a (regular) sieve bootstrap as in Bühlmann (1998) applied to the residuals after nonparametric estimation of the coefficient curves. The second method adds a wild component to the sieve bootstrap. The i.i.d. resampling scheme which is usually applied

\footnotetext{
${ }^{1}$ The explicit form of the matrix $Q_{h}$ is cumbersome and omitted here for the sake of brevity.
} 
to the residuals of the AR fit is replaced by multiplication with a i.i.d. zeromean random variable. This additional step is designed to provided robustness to heteroskedasticity.

\subsubsection{Bootstrap algorithms}

Recall that the errors of our model (4.1) are given by $z_{t}=\sigma_{t} u_{t}$ with $u_{t}=$ $\sum_{j=0}^{\infty} \psi_{j} \epsilon_{t-j}$. The first bootstrap method is designed for the homoskedastic case in which $\sigma_{t}=\sigma$. The focus lies on the dependence structure of the error terms. The sieve bootstrap attempts to approximate the process generating the error terms with an $\operatorname{AR}(p)$ model. This means that we first estimate model (4.1). To the residuals from this estimation we fit an $\operatorname{AR}(p)$ model and, again, obtain the residuals from which we draw the bootstrap errors. We can describe the bootstrap algorithm in six steps:

Algorithm 4.1 (Sieve Bootstrap).

1. Estimate model (4.1) and form a residual series. This means, calculate

$$
\hat{z}_{t}=y_{t}-\mathbf{x}_{t}^{\prime} \widetilde{\boldsymbol{\beta}}(t / n), \quad t=1, \ldots, n,
$$

where the estimate $\widetilde{\boldsymbol{\beta}}(t / n)$ is obtained by bandwidth $\tilde{h}>h$.

2. To the residuals $\hat{z}_{t}$, for $t=1, \ldots, n$, fit an autoregressive model of order $p$ and form the new series of residuals

$$
\hat{\epsilon}_{t}=\hat{z}_{t}-\sum_{j=1}^{p} \hat{\phi}_{j} \hat{z}_{t-j}, \quad t=p+1, \ldots, n
$$

Subtract the mean $\bar{\epsilon}=\frac{1}{n-p} \sum_{t=p+1}^{n} \hat{\epsilon}_{t}$ to form $\tilde{\epsilon}_{t}=\hat{\epsilon}_{t}-\bar{\epsilon}$.

3. Draw randomly with replacement from $\tilde{\epsilon}_{t}$ to obtain $\epsilon_{t}^{*}$.

4. Calculate the bootstrap errors $z_{t}^{*}$ as $z_{t}^{*}=\sum_{j=1}^{p} \hat{\phi}_{j} z_{t-j}^{*}+\epsilon_{t}^{*}$ and generate the bootstrap observations by

$$
y_{t}^{*}=\mathbf{x}_{t}^{\prime} \widetilde{\boldsymbol{\beta}}(t / n)+z_{t}^{*}, \quad t=1, \ldots, n
$$

where $\widetilde{\boldsymbol{\beta}}(t / n)$ is the same as in the first step. 
5. Obtain the bootstrap estimator $\hat{\boldsymbol{\beta}}^{*}(\cdot)$ as defined in (4.6) using the bootstrap series $\left\{y_{t}^{*}\right\}$, with the same bandwidth $h$ as used for the original estimate $\hat{\boldsymbol{\beta}}(\cdot)$.

6. Repeat Steps 2 to $4 B$ times, and let

$$
\hat{q}_{j, \alpha}(\tau)=\inf \left\{u \in \mathbb{R}: \mathbb{P}^{*}\left[\hat{\beta}_{j}^{*}(\tau)-\tilde{\beta}_{j}(\tau) \leq u\right] \geq \alpha\right\}
$$

denote, for all $j=0, \ldots, d$, the $\alpha$-quantile of the $B$ centered bootstrap statistics $\hat{\beta}_{j}^{*}(\tau)-\tilde{\beta}_{j}(\tau)$. These bootstrap quantiles are then used to construct confidence bands as described below.

When generating the bootstrap error process in Step 4, we start the recursion with an arbitrary starting value and wait until stationarity is reached. Following the suggestion in Bühlmann (1998), the order of the approximating $\operatorname{AR}(p)$ model in Step 2 can be selected using the Akaike information criterion. The selection should be done in such a way that the range of possible lag lengths increases with $n$. In practice, we recommend, as in Bühlmann (1998), to select $p$ from a range of possible values in $\left[0, p_{\max }\right]$ with $p_{\max }=o\left(n^{1 / 2}\right)$, for example let $p_{\max }=10 \log _{10}(n)$. Note that in Step 1 of the above algorithm, a different bandwidth is used to perform the nonparametric estimation. Bühlmann (1998) suggests using $\tilde{h}=C h^{5 / 9}$ for $C=0.5,1,2$. Compared to the original bandwidth $h$, this bandwidth is larger and produces an oversmoothed estimate as starting point for the bootstrap procedure. The reason for this is the presence of the asymptotic bias whenever local polynomial estimation is applied. The bias contains the second derivatives of the coefficient functions, which can only be consistently estimated using a larger bandwidth $\tilde{h}$ such that $\tilde{h} n^{1 / 5} \rightarrow \infty$. This is derived in Gasser and Müller (1984). A different approach is undersmoothing which attempts to make the bias asymptotically negligible as in Neumann and Polzehl (1998). An overview about these two approaches is given in Hall and Horowitz (2013). We follow the oversmoothing approach. This has been shown to work well in Bühlmann (1998) as well as in Chapter 2 of this thesis. Therefore, we choose $\tilde{h}$ as in Bühlmann (1998) by setting $\tilde{h}=C h^{5 / 9}$ for $C=0.5,1,2$.

Remark 4.1. Residuals which lie close to the boundary might be problematic as the nonparametric estimator exhibits edge effects. The quality of estimates for points close to the boundary ( $\tau$ close to 0 or 1 ) thus cannot be guaranteed. As a solution, Bühlmann (1998, p. 53) suggests only to resample residuals for points $\tau \in[\delta, 1-\delta]$ for a small $\delta>0$. Formally, this means that in Step 1, residuals $\hat{z}_{t}$ 
are only obtained for $t=[n \delta]+1, \ldots,[n(1-\delta)]$. The remainder of the bootstrap procedure then proceeds with this smaller set of residuals. However, given that we use a local linear estimator and not a local constant approach as in Bühlmann (1998), we expect the effect to be small since the boundary effects are reduced. We investigate the impact of the edge effects in the simulation study.

The sieve bootstrap method, while correctly mimicking the dependence structure, is not valid when the errors are heteroskedastic. In Step 3 of Algorithm 4.1, we draw randomly with replacement from the $\operatorname{AR}(p)$ residuals which leads to bootstrap errors that do not display the same variance pattern as the original residuals. To overcome this, we add a wild component to the sieve bootstrap. This means that a wild bootstrap is applied to the residuals from the autoregressive model and subsequently, the estimated AR parameters are used to construct the bootstrap errors as in Algorithm 4.1. The following bootstrap algorithm shows that the main difference lies in Step 3:

Algorithm 4.2 (Sieve Wild Bootstrap).

1. Identical to Step 1 of Algorithm 4.1.

2. Identical to Step 2 of Algorithm 4.1.

3. Generate $\epsilon_{t}^{*}$ as $\epsilon_{t}^{*}=\xi_{t}^{*} \tilde{\epsilon}_{t}$ with $\xi_{t}^{*} \stackrel{\text { iid }}{\sim} \mathcal{N}(0,1)$.

4. Calculate the bootstrap errors $z_{t}^{*}$ as $z_{t}^{*}=\sum_{j=1}^{p} \hat{\phi}_{j} z_{t-j}^{*}+\epsilon_{t}^{*}$ and generate the bootstrap observations by

$$
y_{t}^{*}=\mathbf{x}_{t}^{\prime} \widetilde{\boldsymbol{\beta}}(t / n)+z_{t}^{*}, \quad t=1, \ldots, n,
$$

where $\widetilde{\boldsymbol{\beta}}(t / n)$ is the same as in the first step.

5. Steps 5 and 6 are again the same as in Algorithm 4.1.

This bootstrap is commonly referred to as sieve wild bootstrap (SWB) and was proposed by Cavaliere and Taylor $(2008,2009)$ as well as Smeekes and Taylor (2012) in the context of unit root testing.

Remark 4.2. Due to the wild component in Step 3 of the sieve wild bootstrap, the residuals are not resampled like in Step 3 of the sieve bootstrap. Therefore, the boundary residuals stay at the boundary and edge effects, if present, are not a problem in this method. 


\subsubsection{Constructing confidence intervals}

To construct pointwise confidence intervals for every $\beta_{j}(\cdot)$, the quantity $\hat{\beta}_{j}^{*}(\tau)-$ $\tilde{\beta}_{j}(\tau)$ is needed for $j=0, \ldots, d$. It is straightforward to determine pointwise twosided confidence intervals for a confidence level of $1-\alpha$. These are exactly the values for every $t$, between which $1-\alpha$ of the deviations fall. Formally, this can be stated as

$$
I_{\alpha}^{*}(\tau)=\left[\hat{\beta}_{j}(\tau)-\hat{q}_{1-\alpha / 2}, \hat{\beta}_{j}(\tau)-\hat{q}_{\alpha / 2}\right]
$$

where $1-\alpha$ is the confidence level and

$$
\hat{q}_{j, \alpha}(\tau)=\inf \left\{u \in \mathbb{R}: \mathbb{P}^{*}\left[\hat{\beta}_{j}^{*}(\tau)-\tilde{\beta}_{j}(\tau) \leq u\right] \geq \alpha\right\}
$$

The quantiles $\hat{q}_{j, \alpha}(\tau)$ are obtained in the last step of one of our bootstrap procedures as given in Algorithms 4.1 and 4.2. From Equation (4.12), it can be seen that the confidence intervals are only valid for a certain point in time. In general, pointwise confidence intervals $I_{j, n, \alpha}^{(p)}$ for $\beta_{j}(\cdot)$ are designed to satisfy ${ }^{2}$

$$
\liminf _{n \rightarrow \infty} \mathbb{P}\left[\left(\beta_{j}(\tau) \in I_{j, n, \alpha}^{(p)}(\tau)\right)\right] \geq 1-\alpha \quad \tau \in(0,1) .
$$

Many interesting research questions, like whether a coefficient remains zero over the whole period or whether there was an upward trend over a certain period of time, cannot be answered with pointwise confidence intervals. The concept of simultaneous confidence bands is needed to answer these questions. In the same notation, for an arbitrary set of time points $G$, the following bands are simultaneous

$$
\liminf _{n \rightarrow \infty}\left[\mathbb{P}\left(\beta_{j}(\tau) \in I_{j, n, \alpha}^{G}(\tau) \quad \forall \tau \in G\right)\right] \geq 1-\alpha
$$

The sieve bootstrap method by Bühlmann (1998) has been shown to provide confidence bands, which are simultaneous within a finite number of neighborhoods $U_{1}, \ldots, U_{p}$. The length of these neighborhoods depends on the bandwidth used for the original estimation, and through the bandwidth it depends on the sample size. The neighborhoods are of the form $U_{i}=\left[\tau_{i}-a h, \tau_{i}+b h\right]$ with $0 \leq a$ and $b<\infty$ such that $G=\cup_{i=1}^{p} U_{i}$.

\footnotetext{
${ }^{2}$ Note that these are non-bootstrap confidence intervals.
} 
Practical implementation follows a three-step procedure similar to the one described in Bühlmann (1998). It is a search based on the ordered deviations, $\hat{\beta}_{j}^{*}(\tau)-\tilde{\beta}_{j}(\tau)$, of bootstrapped estimates from the original estimate, for every coefficient curve $\beta_{j}(\tau), j=0, \ldots, d$. The first step is to construct pointwise quantiles from the deviations:

1. For all $\tau \in G$, for all $j=0, \ldots, d$, obtain pointwise quantiles $\hat{q}_{j, \alpha_{p} / 2}(\tau), \hat{q}_{j, 1-\alpha_{p} / 2}(\tau)$ for varying $\alpha_{p} \in[1 / B, \alpha]$.

2. Choose $\alpha_{s}$ as $\alpha_{s}=\underset{\alpha_{p} \in[1 / B, \alpha]}{\arg \min }\left|\mathbb{P}^{*}\left[\hat{q}_{j, \alpha_{p} / 2}(\tau) \leq \hat{\beta}_{j}^{*}(\tau)-\tilde{\beta}_{j}(\tau) \leq \hat{q}_{j, 1-\alpha_{p} / 2}(\tau) \forall \tau \in G\right]-(1-\alpha)\right|$.

3. Given $\alpha_{s}$ from Step 2, construct the simultaneous confidence bands as

$$
I_{j, n, \alpha_{s}}^{G}(\tau)=\left[\hat{\beta}_{j}(\tau)-\hat{q}_{j, 1-\alpha_{s} / 2}(\tau), \hat{\beta}_{j}(\tau)-\hat{q}_{j, \alpha_{s} / 2}(\tau)\right] \quad \tau \in G .
$$

With the help of this procedure, we build $(1-\alpha)$ confidence bands around every estimated coefficient curve $\hat{\beta}_{j}(\tau)$, which are simultaneous over $G$. A pointwise error $\alpha_{s}$ is found for which a fraction of approximately $(1-\alpha)$ of all centered bootstrap estimates falls within the resulting confidence intervals, for all points of the set $G$. As such, the confidence intervals with pointwise coverage $\left(1-\alpha_{s}\right)$ become simultaneous confidence bands with coverage $(1-\alpha)$. Since this model can have more than one explanatory variable, we have to construct a confidence band for $d$ coefficient curves and the trend function using the above procedure. The dependence on the explanatory variable is denoted in the procedure by the subscript $j$, for $j=0, \ldots, d$. The confidence bands do not have to have equal width, a feature which is valuable, since in finite samples the fluctuations are likely to vary over the time span.

\subsection{Simulation study}

In this section we investigate the properties of the proposed bootstrap method as well as the procedure to generate simultaneous confidence bands with the help of an extensive Monte Carlo study. We choose a data generation process (DGP) which is inspired by our empirical application which is based on a topic from 
environmental economics. We consider several different settings allowing for a wide range of parameter specifications.

\subsubsection{The data generating process}

In the empirical application in Section 4.6 we look at the price development of allowance prices in the European Emissions Trading Scheme (EU ETS). In particular, the relationship between allowance prices and their two main drivers is of interest. As explained in Section 4.6, in theory, one factor should have a negative effect on allowance prices and the other should have a positive effect. In line with the previous literature, we find, however, that the factor with the negative impact is not significant when using linear regression. This is because the effect is insignificant for the first part of our sample and becomes significant after the first few years. Additionally, the positive price determinant displays a two-peak shape in our dataset. We try to mimic both shapes in our simulation study by generating data from the following model:

$$
y_{t}=\beta_{1}(t / n) x_{1, t}+\beta_{2}(t / n) x_{2, t}+u_{t},
$$

where $x_{1, t}$ is given by $x_{1, t}=0.1 x_{t-1}+\xi_{t}$ with $\xi_{t} \sim$ i.i.d. $N(0,1)$ and $x_{2, t}=\zeta_{t}$ with $\zeta_{t} \sim$ i.i.d. $N(0,1)$. The two coefficient functions are given by

$$
\begin{aligned}
& \beta_{1}(t)=1.5 \exp \left(-10(t-0.2)^{2}\right)+1.6 \exp \left(-8(t-0.8)^{2}\right) \\
& \beta_{2}(t)=-0.5 t-0.5 \exp \left(-5(t-0.8)^{2}\right) .
\end{aligned}
$$

For the error term $\left\{u_{t}\right\}$ we consider two cases. In the first case they follow an $\operatorname{ARMA}(1,1)$ process as given by

$$
u_{t}=\phi u_{t-1}+\psi \epsilon_{t-1}+\epsilon_{t} \quad \epsilon_{t} \sim N\left(0, \frac{1-\phi^{2}}{1+\psi^{2}+2 \phi \psi}\right),
$$

where the variance of $\epsilon_{t}$ is such that the signal to noise ratio does not change when we vary $\phi$ and $\psi$. In the second case, in order to introduce conditional heteroskedasticity, we consider $\operatorname{GARCH}(1,1)$ errors.

$$
\begin{aligned}
u_{t} & =\sigma_{t} \epsilon_{t} \\
\sigma_{t}^{2} & =\omega+\alpha_{1} u_{t-1}^{2}+\alpha_{2} \sigma_{t-1}^{2},
\end{aligned}
$$




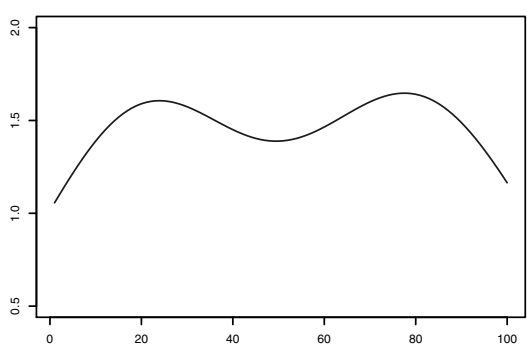

(a) $\beta_{1}(\cdot)$

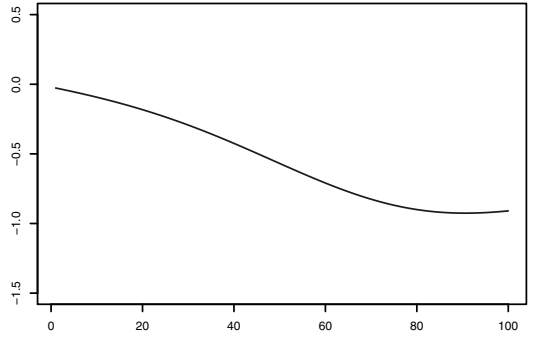

(b) $\beta_{2}(\cdot)$

Figure 4.1: Plot of the coefficient curves $\beta_{1}$ and $\beta_{2}$ used in the simulation study

where $\epsilon_{t} \sim$ i.i.d. $N(0,1)$. We let $\omega=1-\alpha_{1}-\alpha_{2}$ and consider three different $\left(\alpha_{1}, \alpha_{2}\right)$ combinations inspired by the simulation study in Ling et al. (2003): $(0.2,0.7),(0.3,0.6)$ and $(0.4,0.5)$.

Figure 4.1 plots the two coefficient curves which are given by (4.16) and (4.17). It shows that $\beta_{1}(\cdot)$ has two peaks and $\beta_{2}(\cdot)$ starts at zero and then turns negative. As mentioned above, their shape is inspired by the coefficient estimates of the gas and coal price series of the empirical application in Section 4.6.

To obtain the results presented in the next sections, we use 1000 Monte Carlo simulations. To obtain confidence intervals with a $95 \%$ nominal coverage $B=999$ bootstrap replications are used. We apply the local linear estimator with the Epanechnikov kernel which is given by the function $K(x)=\frac{3}{4}\left(1-x^{2}\right) \mathbb{1}_{\{|x| \leq 1\}}$. We also report the average median length of the confidence intervals in parenthesis underneath the respective coverage. Next to pointwise confidence intervals, we also look at simultaneous confidence bands. For simultaneous coverage to be a success, we count the number of successes which means that the trend curve has to lie within the confidence bands for all points of the considered set $G$. We study the performance of the method for the two sets $G_{s u b}$ and $G$ considered by Bühlmann (1998), where $G_{s u b}=U_{1}(h) \cup U_{4}(h)$ and $G=\bigcup_{i=1}^{4} U_{i}(h)$, with $U_{i}(h)=$ $\{(i / 5)-h+j / 100 ; j=0, \ldots,[200 h]\}$. We additionally investigate simultaneous coverage over the whole sample. 


\subsubsection{The $\operatorname{ARMA}(1,1)$ specification}

The first set of results for the DGP with the $\operatorname{ARMA}(1,1)$ error specification is presented in Tables 4.1 through 4.4. They are obtained with a sample size of $n=100$, an oversmoothing parameter choice of $C=2$ and three different bandwidths $h=0.04,0.06,0.08$. Table 4.1 gives coverage probabilities as well as the average median length for the pointwise confidence intervals constructed with our proposed approach. The pointwise coverage represents the fraction of the data points for which the corresponding point on the true parameter curve lies within the confidence bands. Tables 4.2, 4.3 and 4.4 look at the three settings for simultaneous confidence bands: $G, G_{s u b}$ and the whole sample, respectively. In this case, the coverage probability counts the number of times that the true parameter curve lies entirely within the confidence bands (for every point of the considered set). Each table is divided in two parts: the top half displays results for the first coefficient curve $\left(\beta_{1}\right)$ and the bottom half for the second $\left(\beta_{2}\right)$. Recall that both are plotted in Figure 4.1. The left column of each table shows which AR and MA coefficient was used while the top row refers to the bandwidth. For each bandwidth, we present results for the sieve bootstrap (SB) and the sieve wild bootstrap (SWB).

The coverage of the pointwise confidence intervals (Table 4.1) obtained by the sieve bootstrap is close to the nominal level of $95 \%$ in almost all cases, independent of the chosen bandwidth and the degree of autocorrelation. This holds for both coefficient functions. In Tables 4.2 and 4.3 we observe that this is still true for $\beta_{2}$ when we move to simultaneous coverage over the sets $G$ and $G_{s u b}$. Looking at the same values for $\beta_{1}$, the coverage drops as the bandwidth gets larger. The coverage drops to $75 \%$ for $G_{s u b}$ and $76 \%$ for $G$ in the case of the AR errors with $\phi=0.5$ for the largest considered bandwidth. For the smallest bandwidth, the coverage for $\beta_{1}$ varies from $93 \%$ to $97 \%$. The confidence intervals get more narrow as the bandwidth increases which could explain the drop in coverage. In addition, the sensitivity with respect to the bandwidth observed for $\beta_{1}$ is not unexpected given the more complex shape of the parameter curve compared to $\beta_{2}$. In Table 4.4, the same pattern arises when we consider simultaneity over the whole sample, while the confidence intervals become slightly wider compared to the previous tables to account for the substantially larger set of values considered for the simultaneity.

The sieve bootstrap performs considerably better than the sieve wild bootstrap. This is also not surprising since the advantage of the added wild component is to account for heteroskedasticity in the data, but the level of the coverage is 


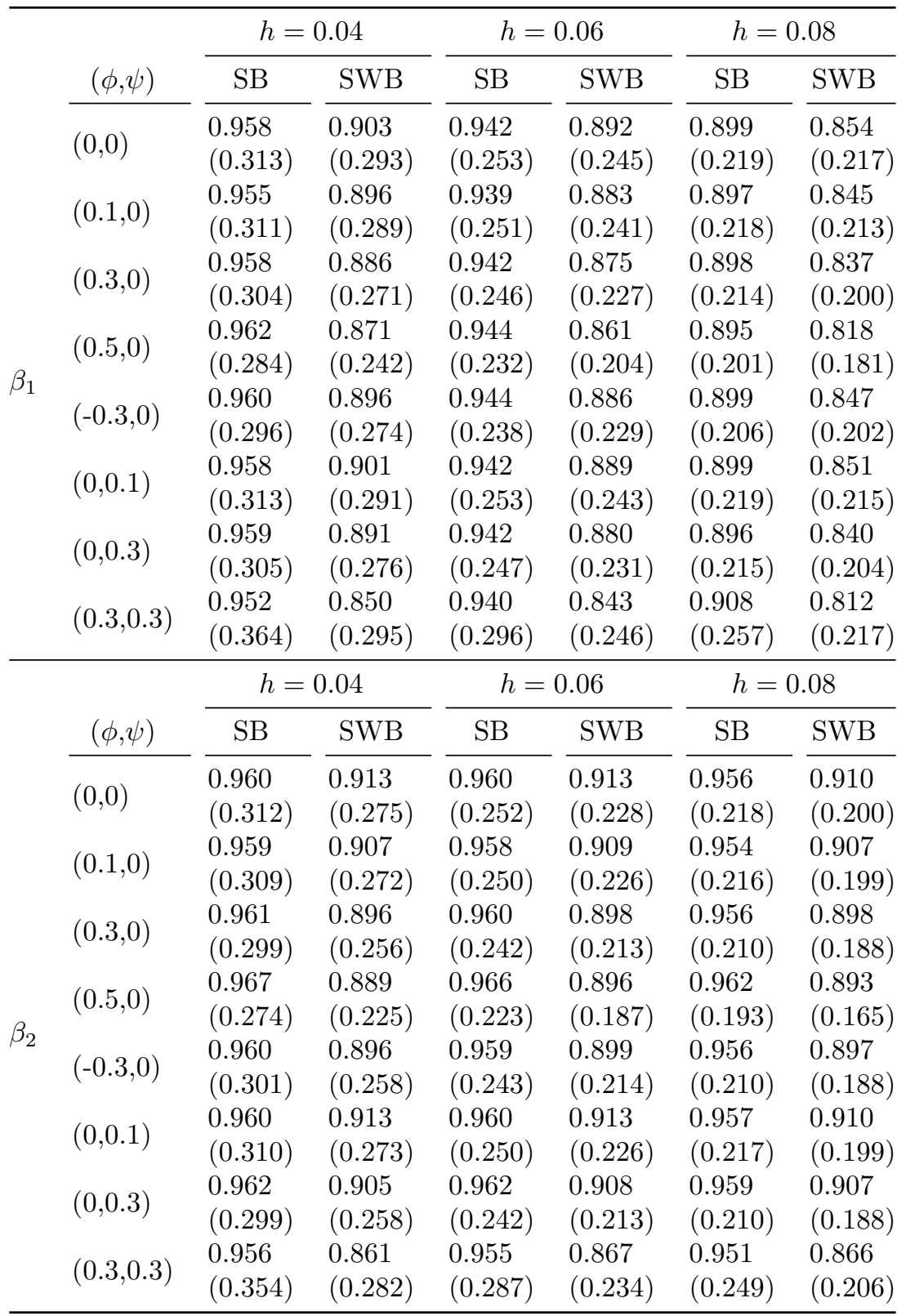

Table 4.1: Pointwise coverage probabilities, $\operatorname{ARMA}(1,1)$ 


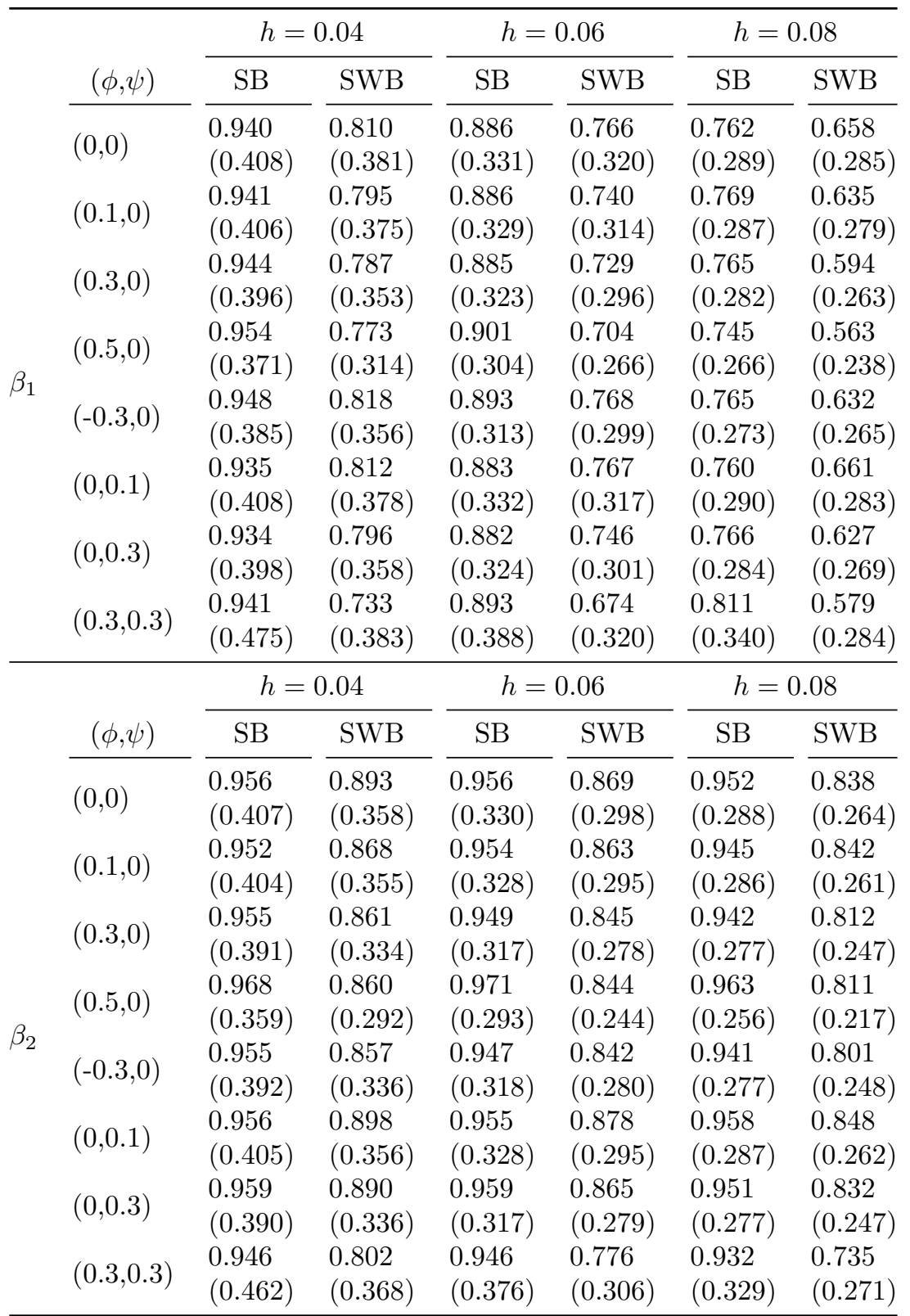

Table 4.2: Coverage probabilities for $G_{s u b}, \operatorname{ARMA}(1,1)$ 


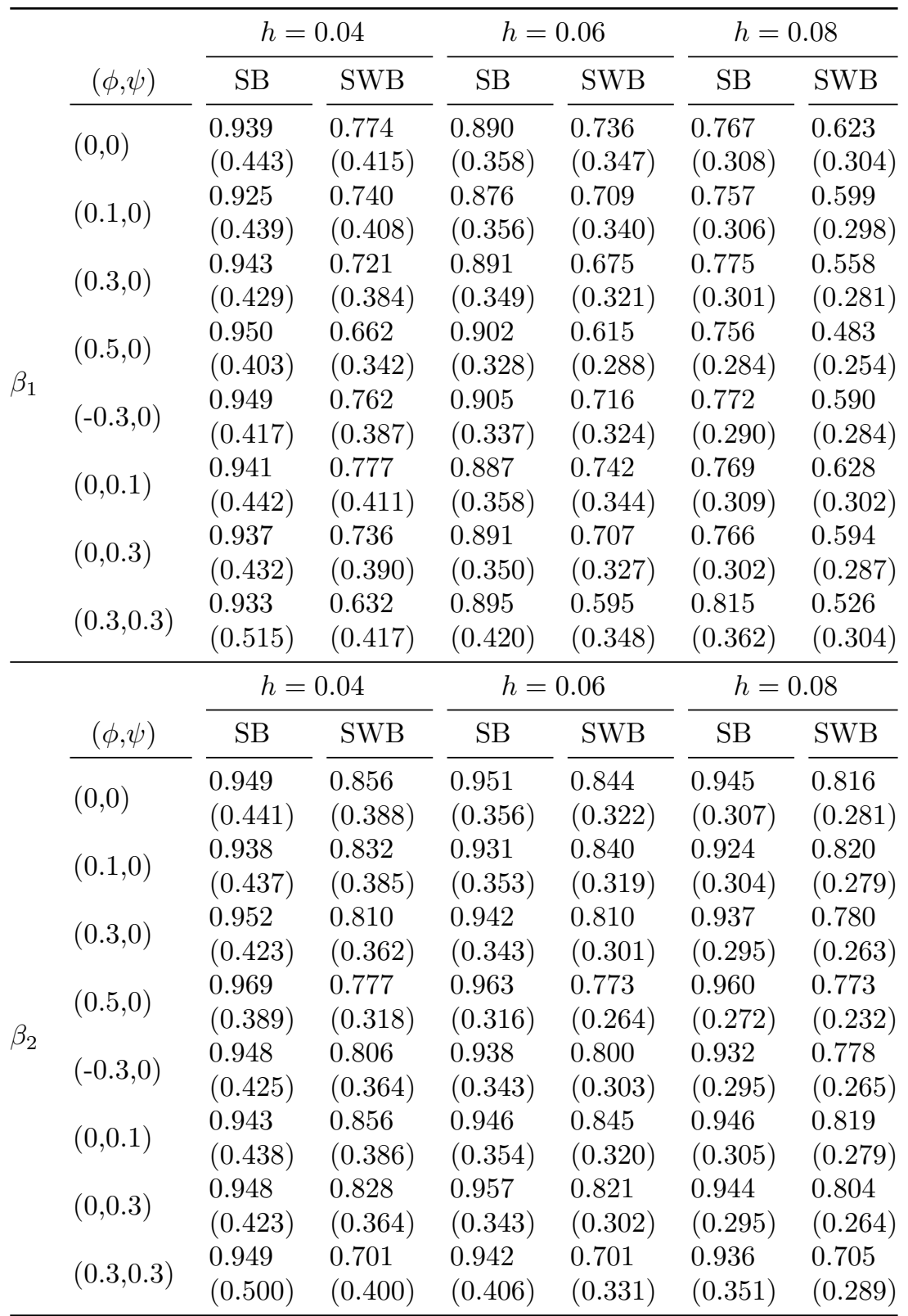

Table 4.3: Coverage probabilities for $G, \operatorname{ARMA}(1,1)$ 


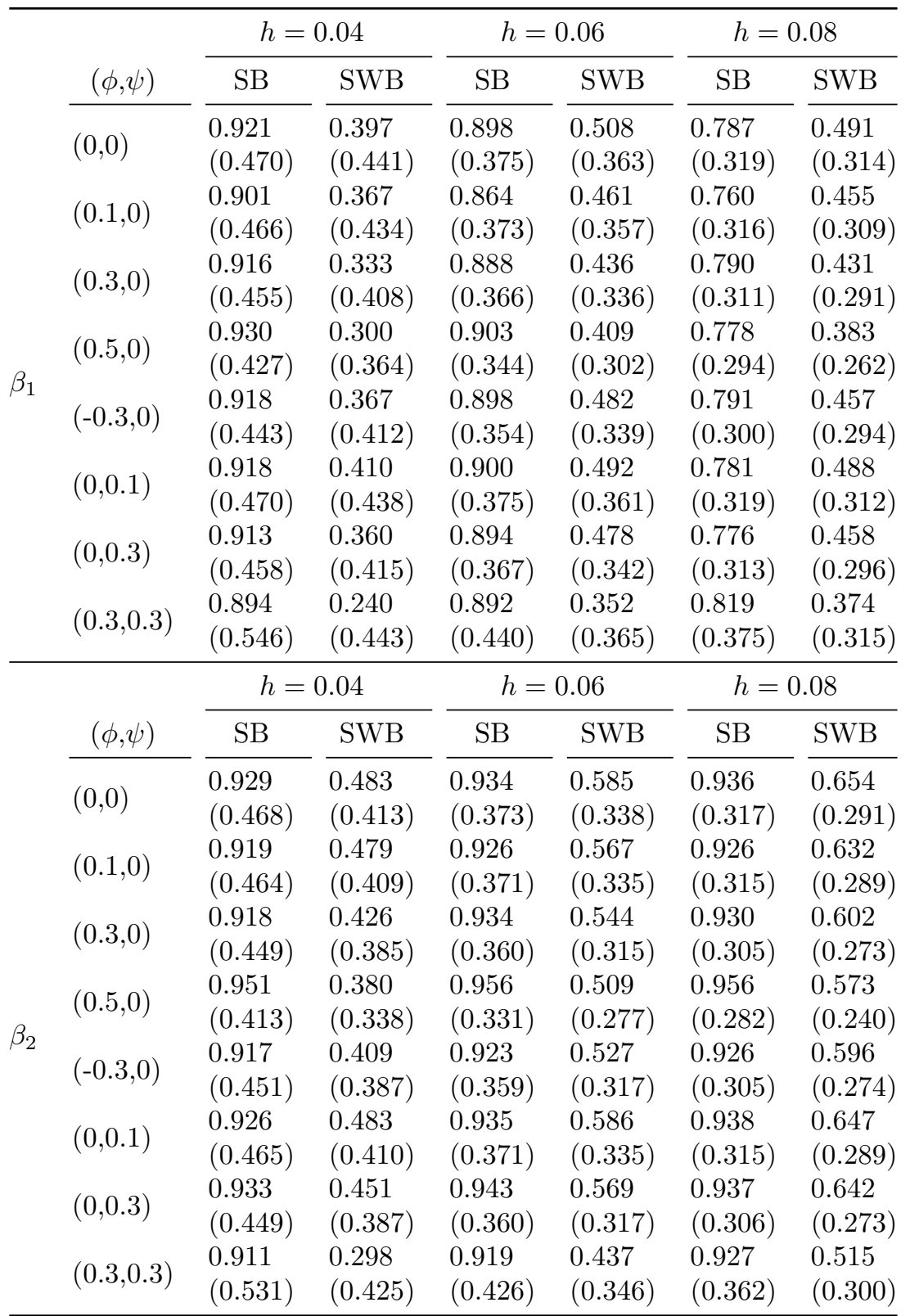

Table 4.4: Coverage probabilities for the whole sample, ARMA(1,1) 


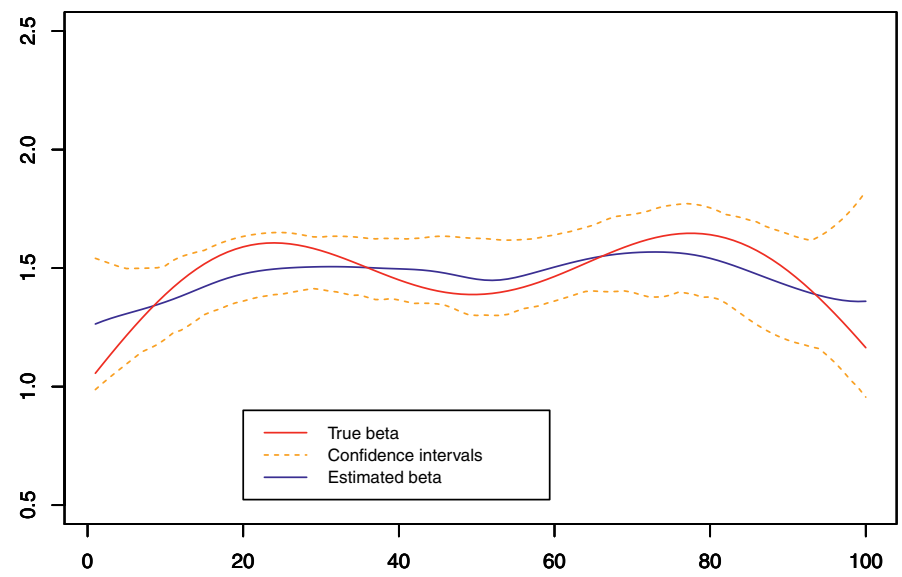

(a) Sieve bootstrap

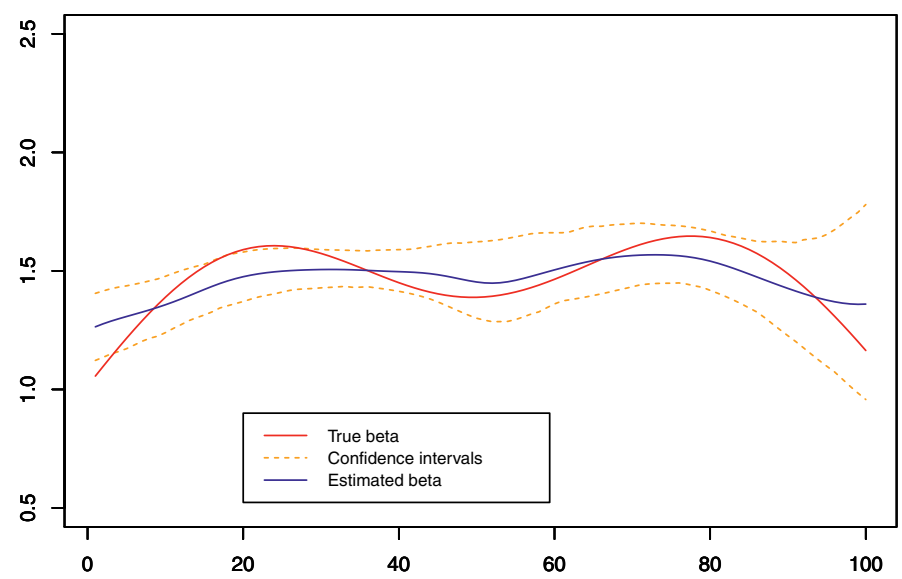

(b) Sieve wild bootstrap

Figure 4.2: Comparison of the two bootstrap methods for $\beta_{1}(\cdot)$ and $h=0.08$. The data was generated with the $\operatorname{AR}(1)$ specification $(\phi=0.5)$.

surprisingly low. Already for the pointwise intervals, a coverage of not more than $91 \%$ of data points is achieved. For simultaneous confidence bands, the values drop as low as $70 \%$ for $G$ and $24 \%$ for the whole sample. Recall that in this case we count the number of draws from the DGP in which the true parameter curve can be embedded in the confidence bands (for all points in $G$ or for the entire sample). We observe in all tables a difference in median lengths between the two bootstrap 


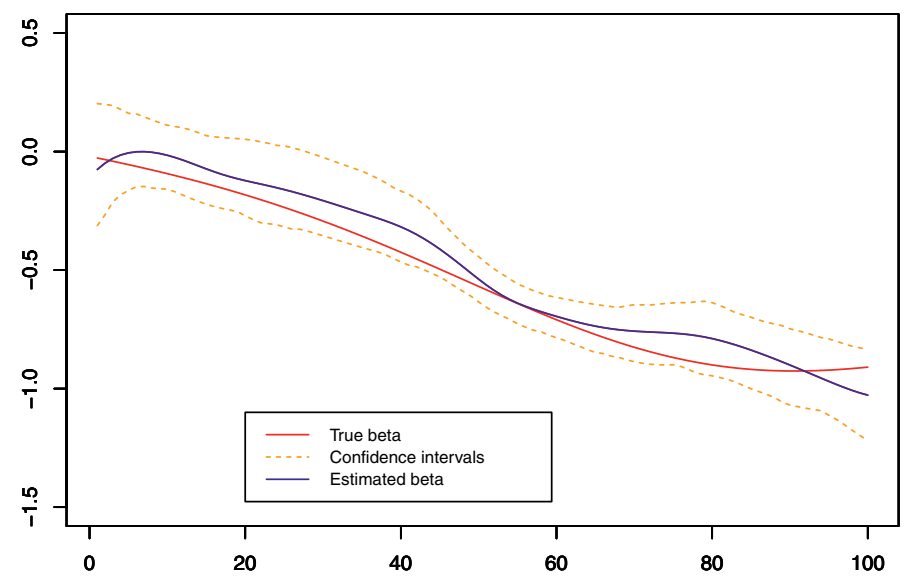

(a) Sieve bootstrap

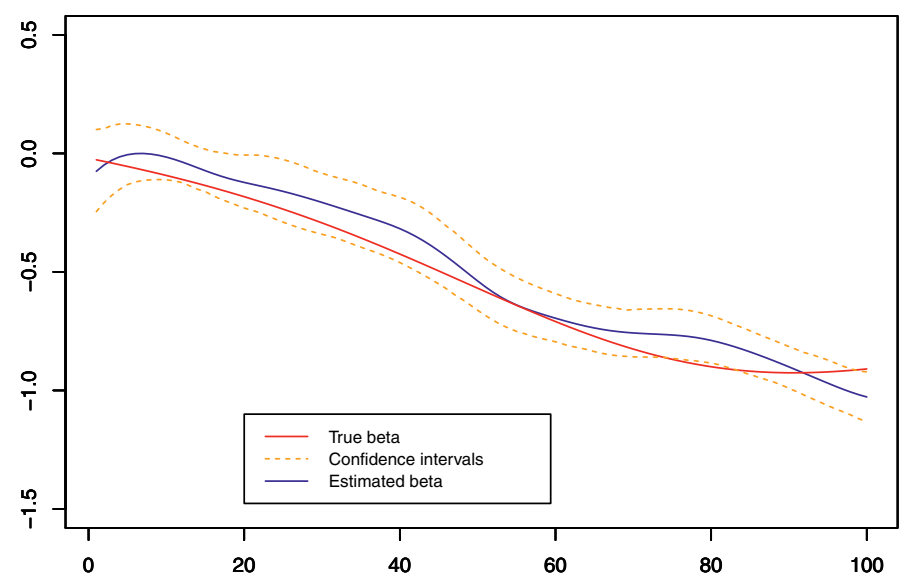

(b) Sieve wild bootstrap

Figure 4.3: Comparison of the two bootstrap methods for $\beta_{2}(\cdot)$ and $h=0.08$. The data was generated with the $\operatorname{AR}(1)$ specification $(\phi=0.5)$.

methods. The sieve bootstrap produces slightly wider confidence intervals, which is a result that holds throughout all considered specifications. It is, however, more pronounced for $\beta_{2}$ than for $\beta_{1}$. In addition, the difference becomes larger for simultaneous confidence bands than for the pointwise intervals. Although the differences in lengths do not seem substantial at a first glance, we observe from plotting several examples that they play an important role. 
In Figures 4.2 and 4.3, we plot two examples where the sieve bootstrap provides successful coverage over the whole sample, while the wild version does not. ${ }^{3}$ Figure 4.2 refers to $\beta_{1}$ and Figure 4.3 to $\beta_{2}$. In both figures, Panel (a) shows the sieve bootstrap results and Panel (b) the sieve wild. These examples are generated using a random draw from the ARMA specification with an AR coefficient of 0.5 . The true parameter curve is plotted in red, while the estimate - produced with a bandwidth parameter of 0.08 - is given in blue. Confidence bands which are simultaneous over the whole sample are presented as the orange dotted lines.

This is a case where the difference in coverage between the two methods is substantial. From the graphs we can observe that the sieve wild confidence bands are overall more narrow. In particular, in areas where the estimated parameter lies far above or below the true value, the sieve bootstrap seems to be able to capture this by a widening of the confidence bands. This is not the case for the sieve wild bootstrap.

In addition, the widening of the bands which is visible for the sieve bootstrap at the beginning and the end of the sample, is only present at the end of the sample for the wild counterpart. As mentioned in Remark 4.1, results produced for the beginning as well as the end of the sample have to be interpreted with care, because the truncation of the nonparametric estimator can distort the results. To exclude that this is a driving factor, results where a fraction of $h * n$ observations at the beginning and end are left out are reported in the Appendix of this chapter (Table 4.15). Leaving out these observations leads to small improvements in coverage but it cannot be seen as the single explanation driving previous results.

Overall, we can conclude from these results that the difference in widths, which did not seem as striking in the tables, seems to be the most important factor explaining the sometimes substantial differences in coverage.

In a final set of results, we investigate the role of the oversmoothing parameter $C$. Here, we only consider a limited number of ARMA specifications $((0,0)$, $(0.3,0),(0,0.3))$ and we restrict ourselves to one bandwidth $(h=0.04)$. The results are presented in Table 4.5. The top part of the table shows pointwise coverage and the bottom part simultaneous coverage over $G_{s u b}$ for an increasing value of $C$. In addition to the previous choice of $C=2$, we consider $C=0.5$ and $C=1$ as in Bühlmann (1998). For ease of comparison, the final two columns repeat the values from Tables 4.1 and 4.2 , respectively.

\footnotetext{
${ }^{3}$ Successful coverage means that the true parameter curve (plotted as the solid red curve) lies completely within the confidence bands (plotted as the dotted orange lines).
} 
With increasing $C$ the confidence intervals become wider and thus, the coverage increases. For the sieve bootstrap, values of 1 and 2 both produce accurate coverage when it comes to pointwise confidence intervals. For the sieve wild bootstrap, the largest considered value is preferred. In the case of simultaneous confidence bands, both methods work best with an oversmoothing parameter of $C=2$.

\subsubsection{The $\operatorname{GARCH}(1,1)$ specification}

For the $\operatorname{GARCH}(1,1)$ error specification, we see in Tables 4.6 through 4.9 that the performance is similar to that of the previous specification. In general, the sieve bootstrap outperforms the sieve wild version for all considered specifications. Table 4.6 shows the pointwise coverage results for the three considered bandwidths. While the sieve bootstrap has a coverage close to the nominal level, the sieve wild version achieves a maximum coverage of around 91\%. As in the ARMA specification, the coverage of both methods drops for $\beta_{1}$ when the largest bandwidth is considered. The maximum coverage lies between 83 and $89 \%$. In the case of $\beta_{2}$, this drop cannot be observed.

Moving to Tables 4.7 and 4.8 , we note that the coverage of the simultaneous confidence bands (for both $G$ and $G_{\text {sub }}$ ) drops to a level of around 90-91\%, which is achieved by the SB. The wild counterpart displays coverage probabilities of around $80-83 \%$. Again, the coverage for $\beta_{1}$ is more sensitive to the choice of bandwidth. While for $\beta_{2}$ it remains at around $90 \%$ (for the SB) or $80 \%$ (for the SWB) irrespective of the bandwidth, coverage for $\beta_{1}$ can be as low as $71 \%$ (for the SB) or even $57 \%$ (for the SWB) for $h=0.08$. For the medium bandwidth, the SB is closer to the nominal level with a coverage of around $85-88 \%$ compared to $71-76 \%$ for the SWB. Coverage probabilities for both methods in the small bandwidth case lie close to the coverage achieved for $\beta_{2}$.

In Table 4.9, we display the results for simultaneity over the whole sample. The sieve bootstrap coverage for small to medium bandwidths lies at around $80-87 \%$. For $h=0.08$, coverage for $\beta_{1}$ drops to $70-74 \%$. The sieve wild bootstrap shows very low coverage probabilities while being highest for $\beta_{2}$ (with $h=0.08$ ).

Regarding the lengths of the confidence intervals, the same pattern arises as in the previous specification. The sieve bootstrap intervals are always wider than their wild bootstrap counterparts offering a potential explanation for the lower 


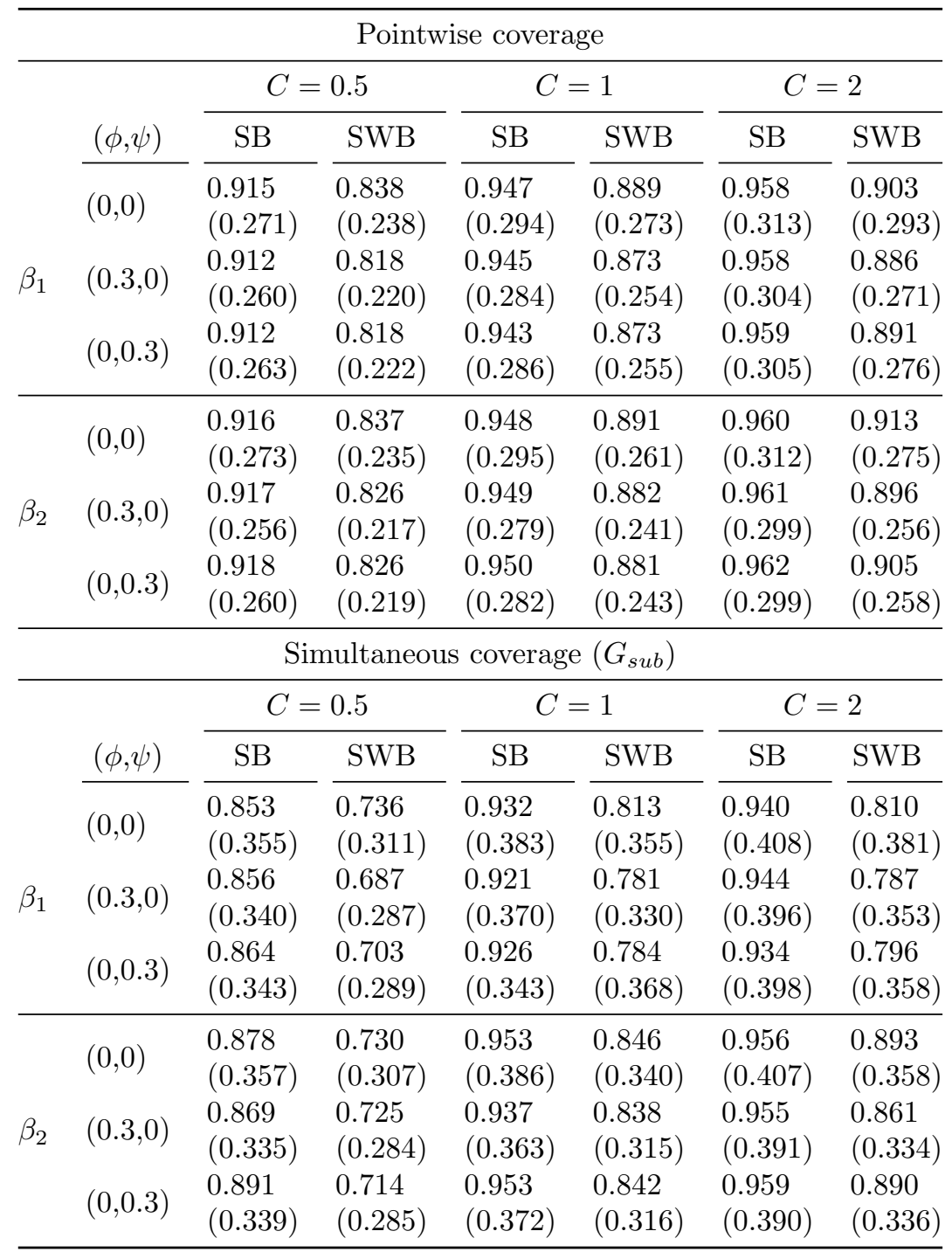

Table 4.5: Coverage probabilities for varying $C, \operatorname{ARMA}(1,1), h=0.04$ 


\begin{tabular}{|c|c|c|c|c|c|c|c|}
\hline & \multirow[b]{2}{*}{$\left(\alpha_{1}, \alpha_{2}\right)$} & \multicolumn{2}{|c|}{$h=0.04$} & \multicolumn{2}{|c|}{$h=0.06$} & \multicolumn{2}{|c|}{$h=0.08$} \\
\hline & & SB & SWB & SB & SWB & SB & SWB \\
\hline \multirow{3}{*}{$\beta_{1}$} & $(0.2,0.7)$ & $\begin{array}{l}0.954 \\
(0.302)\end{array}$ & $\begin{array}{l}0.895 \\
(0.273)\end{array}$ & $\begin{array}{l}0.938 \\
(0.244)\end{array}$ & $\begin{array}{l}0.883 \\
(0.229)\end{array}$ & $\begin{array}{l}0.890 \\
(0.211)\end{array}$ & $\begin{array}{l}0.838 \\
(0.204)\end{array}$ \\
\hline & $(0.3,0.6)$ & $\begin{array}{l}0.952 \\
(0.296)\end{array}$ & $\begin{array}{l}0.897 \\
(0.261)\end{array}$ & $\begin{array}{l}0.937 \\
(0.239)\end{array}$ & $\begin{array}{l}0.883 \\
(0.220)\end{array}$ & $\begin{array}{l}0.885 \\
(0.207)\end{array}$ & $\begin{array}{l}0.836 \\
(0.196)\end{array}$ \\
\hline & $(0.4,0.5)$ & $\begin{array}{l}0.952 \\
(0.288)\end{array}$ & $\begin{array}{l}0.900 \\
(0.249)\end{array}$ & $\begin{array}{l}0.937 \\
(0.233)\end{array}$ & $\begin{array}{l}0.885 \\
(0.211)\end{array}$ & $\begin{array}{l}0.882 \\
(0.203)\end{array}$ & $\begin{array}{l}0.834 \\
(0.189)\end{array}$ \\
\hline \multirow{3}{*}{$\beta_{2}$} & $(0.2,0.7)$ & $\begin{array}{l}0.953 \\
(0.302)\end{array}$ & $\begin{array}{l}0.899 \\
(0.251)\end{array}$ & $\begin{array}{l}0.953 \\
(0.243)\end{array}$ & $\begin{array}{l}0.902 \\
(0.210)\end{array}$ & $\begin{array}{l}0.951 \\
(0.210)\end{array}$ & $\begin{array}{l}0.900 \\
(0.186)\end{array}$ \\
\hline & $(0.3,0.6)$ & $\begin{array}{l}0.951 \\
(0.295)\end{array}$ & $\begin{array}{l}0.900 \\
(0.238)\end{array}$ & $\begin{array}{l}0.952 \\
(0.238)\end{array}$ & $\begin{array}{l}0.902 \\
(0.200)\end{array}$ & $\begin{array}{l}0.950 \\
(0.206)\end{array}$ & $\begin{array}{l}0.900 \\
(0.178)\end{array}$ \\
\hline & $(0.4,0.5)$ & $\begin{array}{l}0.951 \\
(0.289)\end{array}$ & $\begin{array}{l}0.904 \\
(0.226)\end{array}$ & $\begin{array}{l}0.953 \\
(0.233)\end{array}$ & $\begin{array}{l}0.907 \\
(0.190)\end{array}$ & $\begin{array}{l}0.951 \\
(0.202)\end{array}$ & $\begin{array}{l}0.903 \\
(0.169)\end{array}$ \\
\hline
\end{tabular}

Table 4.6: Pointwise coverage probabilities, $\operatorname{GARCH}(1,1)$

coverage. In all considered cases, we see only minor differences between the three chosen GARCH parameter specifications in both length and coverage. ${ }^{4}$

The lower performance of the sieve bootstrap in this specification compared to the previous one is an expected result given that the method is not designed for heteroskedastic data. The fact that it nevertheless outperforms its wild extension is surprising. The confidence intervals constructed with the latter method are too narrow resulting in coverage probabilities that are too far below the nominal level. These results suggest that simply extending the sieve bootstrap with a wild component cannot be recommended in this case. Due to the existence of various bootstrap methods as well as several combinations and extensions thereof, it is tempting to simply find a version that is robust in the case at hand. However, our results stress that bootstrap methods should not simply be extended without a careful investigation of the performance in the given situation.

\subsubsection{Bandwidth selection methods}

Given that the previous results, or at least parts of it, show a dependence of the bootstrap's performance on the bandwidth, we look at the data-driven band-

\footnotetext{
${ }^{4}$ In the Appendix to this chapter we report some additional results for a combination of the two error structures: an ARMA-GARCH specification. We also increase the sample size of the pure ARMA specification to 300. All additional results show similar patterns than the ones presented here.
} 


\begin{tabular}{|c|c|c|c|c|c|c|c|}
\hline & & $h=$ & 0.04 & $h=$ & 0.06 & $h=$ & .08 \\
\hline & $\left(\alpha_{1}, \alpha_{2}\right)$ & SB & SWB & $\mathrm{SB}$ & SWB & SB & SWB \\
\hline & $(0.2,0.7)$ & 0.912 & 0.790 & 0.882 & 0.740 & 0.745 & 0.636 \\
\hline & & $(0.397)$ & $(0.354)$ & $(0.321)$ & $(0.299)$ & $(0.280)$ & $(0.267)$ \\
\hline$\beta_{1}$ & $(\cap 2 \Omega 6$ & 0.901 & 0.787 & 0.871 & 0.747 & 0.733 & 0.624 \\
\hline$\rho_{1}$ & 0.0 & $(0.390)$ & $(0.338)$ & $(0.316)$ & $(0.286)$ & $(0.275)$ & $(0.257)$ \\
\hline & 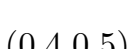 & 0.895 & 0.801 & 0.871 & 0.756 & 0.713 & 0.613 \\
\hline & $(0.4,0.5)$ & $(0.383)$ & $(0.323)$ & $(0.310)$ & $(0.275)$ & $(0.270)$ & $(0.247)$ \\
\hline & $(0207)$ & 0.912 & 0.829 & 0.909 & 0.818 & 0.901 & 0.787 \\
\hline & $(0.2, \mathrm{C}$ & $(0.396)$ & $(0.327)$ & $(0.320)$ & $(0.274)$ & $(0.279)$ & $(0.245)$ \\
\hline$\beta$ & $(0206)$ & 0.911 & 0.829 & 0.904 & 0.804 & 0.895 & 0.778 \\
\hline$p_{2}$ & (c) & $(0.390)$ & $(0.310)$ & $(0.315)$ & $(0.261)$ & $(0.274)$ & $(0.234)$ \\
\hline & $(0405)$ & 0.903 & 0.842 & 0.904 & 0.811 & 0.894 & 0.785 \\
\hline & $(0.4,0.5)$ & $(0.383)$ & $(0.294)$ & $(0.310)$ & $(0.248)$ & $(0.269)$ & $(0.223)$ \\
\hline
\end{tabular}

Table 4.7: Coverage probabilities for $G_{s u b}, \operatorname{GARCH}(1,1)$

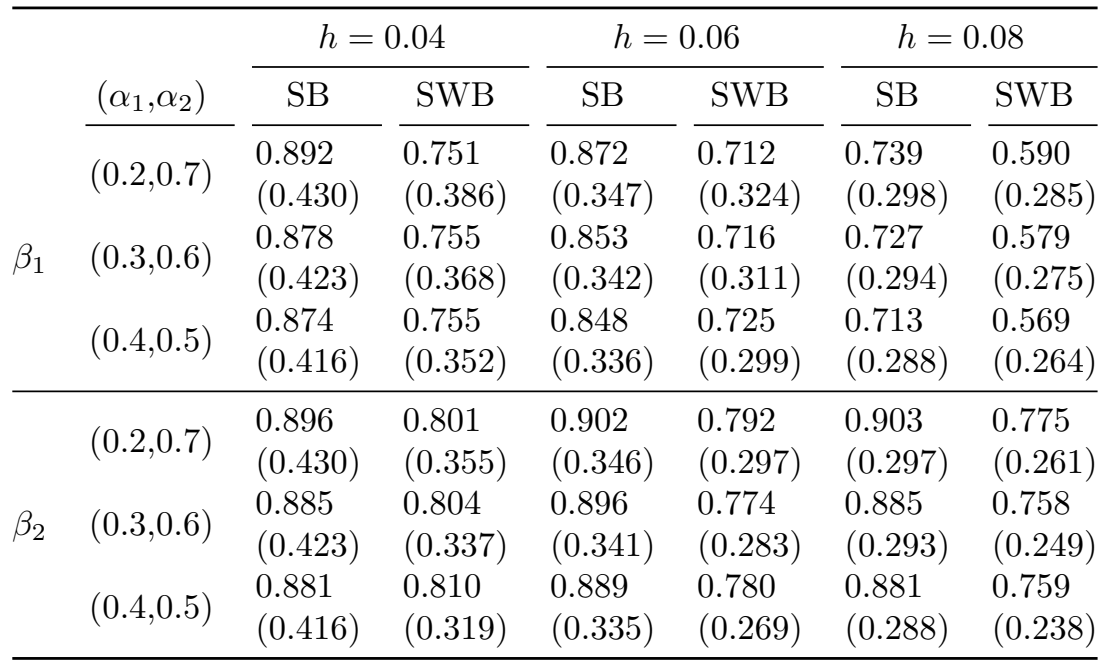

Table 4.8: Coverage probabilities for $G$ 


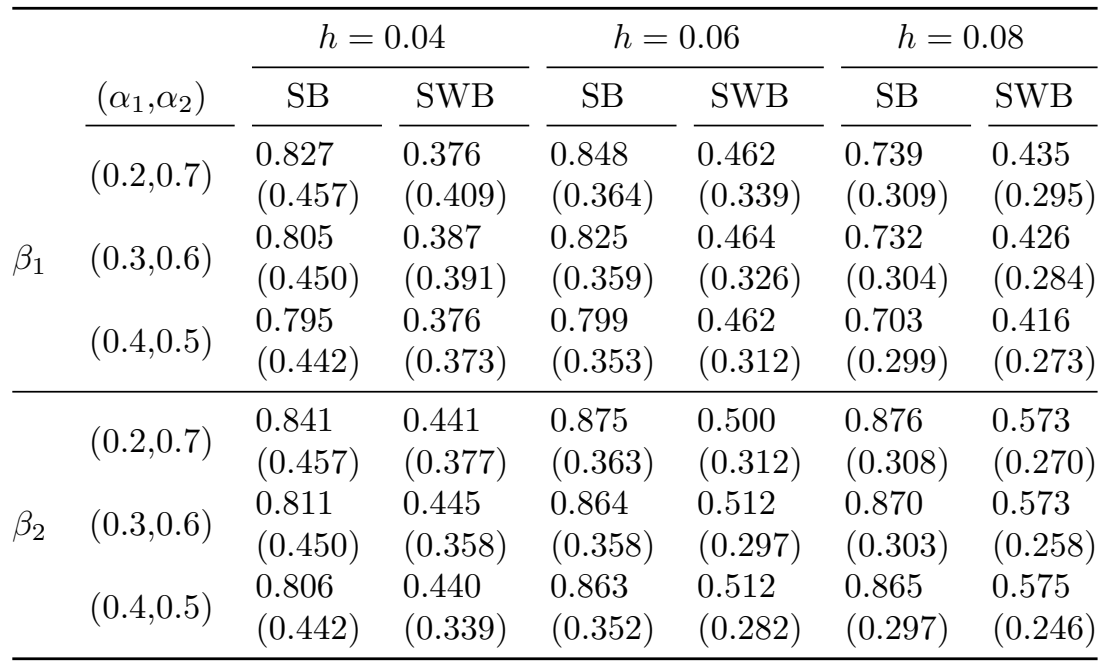

Table 4.9: Coverage probabilities for the whole sample

width selection methods presented in Section 4.3.2 as a final step of this simulation study. We generate 1000 samples from each of the following four error specifications: white noise, $\operatorname{AR}(1)$ with $\phi=0.3, \operatorname{AR}(1)$ with $\phi=-0.3$ and $\operatorname{GARCH}(1,1)$ with $\alpha_{1}=0.2$ and $\alpha_{2}=0.7$. For each draw, we select the optimal bandwidth chosen by the four different methods: Cross Validation (CV), Generalized Cross Validation (GCV), the Akaike selection criterion (AIC) and Leave- $(2 l+1)$-out Cross Validation. We apply the latter method with $l=2,4,6$ and denote them by L2O, L4O and L6O, respectively. The set of possible bandwidths to select from in each case ranges from 0.02 to 0.12 in steps of 0.001 .

The average selected bandwidths as well as the standard deviations are reported in Table 4.10. The left two columns present the white noise case. Here, bandwidths between 0.076 (Generalized Cross validation) and 0.103 (Leave- $(2 l+1)$ out Cross Validation with $l=6$ ) are chosen. The middle two columns show that the results are very similar for the $\mathrm{AR}(1)$ errors. Although it has been shown that cross validation produces a bandwidth that is too small in the presence of positive correlation (Chu and Marron, 1991), the selected bandwidths are only slightly smaller compared to the independent case. The differences between the two columns are around 0.002-0.003, which is approximately the magnitude of the standard deviations. The same holds for the $\mathrm{AR}(1)$ case with negative correlation where theory suggests that the chosen bandwidth is too large. Comparing to the 
results for positive correlation, the values are slightly larger. However, they are smaller than for white noise.

In the final two columns we see that for the $\operatorname{GARCH}(1,1)$ case, the selected bandwidths are substantially smaller and have a smaller standard deviation. As in the two other cases, Cross Validation selects the smallest bandwidth, followed by Generalized Cross Validation. Leave- $(2 l+1)$-out Cross Validation chooses the largest bandwidths with $l=6$.

Overall, the chosen bandwidths often lie close to the largest value we considered in our simulation study which is 0.08 . For the second, less intricate parameter curve, this value resulted in accurate coverage of the sieve bootstrap. For the first parameter curve, a smaller bandwidth parameter produced better coverage. Although a bandwidth matrix consisting of different bandwidths for each parameter curve is sometimes considered in the theoretical literature, in practice, a data driven bandwidth selection method to determine such a matrix is computationally cumbersome. This is why we consider the same bandwidth parameter for both curves.

Combining these results suggests to use a smaller bandwidth in practice than the one selected by the data-driven methods. While a smaller bandwidth might not produce an optimal approximation to coefficient curves with low variations, it nevertheless led to good coverage for such cases in our simulation study. For more difficult to estimate coefficient curves, a small bandwidth is preferable and it produced superior results when looking at coverage.

This shows that blindly relying on data-driven bandwidth selection methods can be problematic in practice. We recommend, in addition to such selection

\begin{tabular}{|c|c|c|c|c|c|c|c|c|}
\hline \multicolumn{9}{|c|}{ Bandwidth selection } \\
\hline \multirow[b]{2}{*}{ Method } & \multicolumn{2}{|c|}{$(\phi, \psi)=(0,0)$} & \multicolumn{2}{|c|}{$\phi=0.3$} & \multicolumn{2}{|c|}{$\phi=-0.3$} & \multicolumn{2}{|c|}{ GARCH } \\
\hline & $\mu$ & $(\sigma)$ & $\mu$ & $(\sigma)$ & $\mu$ & $(\sigma)$ & $\mu$ & $(\sigma)$ \\
\hline $\mathrm{CV}$ & 0.085 & $(0.024)$ & 0.083 & $(0.023)$ & 0.084 & $(0.022)$ & 0.082 & $(0.025)$ \\
\hline GCV & 0.076 & $(0.028)$ & 0.074 & $(0.028)$ & 0.076 & $(0.027)$ & 0.072 & $(0.027)$ \\
\hline AIC & 0.095 & $(0.018)$ & 0.093 & $(0.017)$ & 0.094 & $(0.017)$ & 0.093 & $(0.019)$ \\
\hline $\mathrm{L} 2 \mathrm{O}$ & 0.092 & $(0.021)$ & 0.090 & $(0.022)$ & 0.091 & $(0.020)$ & 0.089 & $(0.022)$ \\
\hline $\mathrm{L} 4 \mathrm{O}$ & 0.099 & $(0.019)$ & 0.096 & $(0.020)$ & 0.096 & $(0.019)$ & 0.094 & $(0.024)$ \\
\hline L6O & 0.103 & $(0.017)$ & 0.101 & $(0.018)$ & 0.101 & $(0.017)$ & 0.101 & $(0.018)$ \\
\hline
\end{tabular}

Table 4.10: Mean $(\mu)$ and stand deviation $(\sigma)$ of selected bandwidths 
methods, to perform a robustness analysis of the main results using different bandwidths.

\subsection{Application to the EU Emissions Trading Scheme}

Interesting areas for potential applications are, for instance, climatology and environmental economics. In these fields, it is quite common that relationships are varying smoothly over time rather than being subject to abrupt changes. Here, we would like to focus on an application to CO2 emission trading which is an important topic in environmental economics and politics. In particular, the European system - the EU ETS which is short for European Emissions Trading Scheme - is the largest and oldest cap-and-trade program.

\subsubsection{Background and related literature}

In the EU ETS, regulated firms and other participants can buy and sell permits that can be used to match emissions of 1 metric ton of $\mathrm{CO} 2$ or $\mathrm{CO} 2$ equivalent. These permits are called emission allowances (EUAs). The EU ETS covers around $45 \%$ of all greenhouse gas emissions of the EU. The main sector which is regulated by the scheme is the power sector. Energy intensive industries and the aviation sector are also part of the current version of the scheme. Since its implementation in 2005, the EU ETS went through two compliance phases and is currently in its third phase which began in 2013 and ends in 2020. Phase I, which ran from 2005 to 2007, was the trial phase and since then, the system underwent major reforms to become what it is today.

Despite the constant evaluation of the functioning and updating of rules, the market suffered from a long period of low prices which was seen by many as failure of the system to achieve its goal of establishing an efficient price of carbon. Figure 4.4 plots the daily price path of allowances since January $2008 .^{5}$ We decide to display December futures which are commonly used for empirical studies and which are frequently traded contracts. In 2011, there was a large price drop and the

\footnotetext{
${ }^{5}$ We do not plot the price development in Phase I where the fact that banking allowances from Phase I to Phase II was not allowed caused a huge price collapse at the end of Phase I. This led the price to approach zero towards the end of the period. Starting in Phase II, the no-banking rule was abolished such that allowances from previous phases remain valid. As we can observe in Figure 4.4, this made the transition between phases smoother.
} 
subsequent low prices lay between 5 and $10 €$ per ton of CO2 until 2017. Although the cause of this remains a puzzle to researchers and politicians, a lack of credibility and an oversupply of allowances in the system are among the potential reasons (Edenhofer, 2014; Koch et al., 2014). Similarly, the recent upward trend has received a lot of attention. A new reform which is designed to make the supply of allowances more flexible and the system as a whole more resilient to future shocks seems to be showing its desired effect even before its official introduction in January 2019. These are the most important developments of allowance prices.

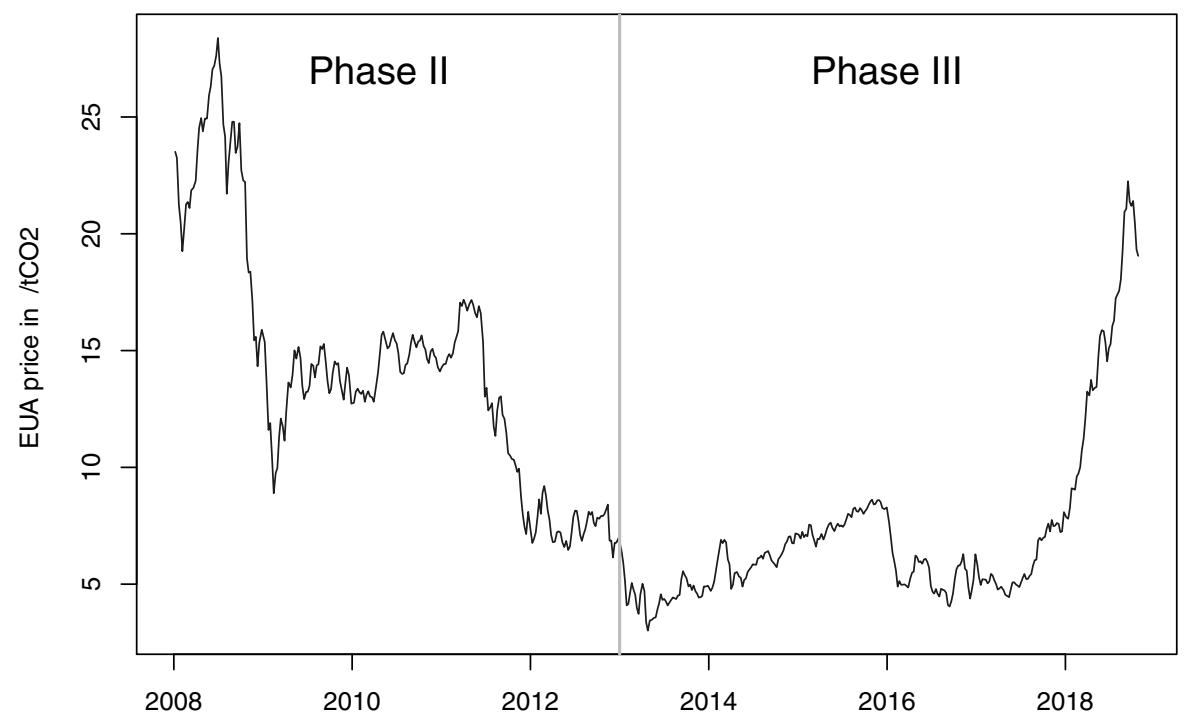

Figure 4.4: EUA December futures divided into compliance periods (data from EEX Leipzig)

An interesting area of research within this context is to study the impact of fundamental price drivers on the allowance price. Economic theory identifies clear price drivers among which the most important ones are the coal and the gas price. The power sector is the main sector in the EU ETS and therefore, it is responsible for the identification of the main price determinants. In this sector, firms can switch from coal to gas for electricity generation. In theory, due to the fuel switching relationship, the coal price is expected to have a negative effect on allowances prices. In contrast, gas should have a positive effect. Using coal in electricity generation produces much more emissions than using gas. Thus, an increase in the price of coal, ceteris paribus, leads to a switch from coal to gas which 
reduces emissions and simultaneously, it decreases the demand for allowances. Other price driving factors are e.g. economic activity which should have a positive effect on allowance prices. Electricity consumption and therefore emissions will increase with an increase in economic activity. In contrast, electricity generation from renewable sources should have a negative effect due to cleaner technologies, resulting in less demand for allowances. The effect of temperature on allowance prices is expected to be mainly due to increased use of heating during winter and therefore, theory predicts a negative effect.

Although market fundamentals should have a major effect on allowance prices, a study of the related literature shows that empirical evidence is mixed. Previous studies indicate that standard approaches, like linear regression models, need to be adapted by splitting the sample, including breaks or dummy variables in order to improve their findings. This is summarized in a review of the empirical literature on the EU ETS by Friedrich et al. (2019).

Linear models have been used in e.g. Hintermann (2010), Koch et al. (2014), Aatola et al. (2013) and many others. The question of whether it might be more appropriate to account for potential time-variation when modeling the relationship between allowance prices and fundamentals has not only been raised in Friedrich et al. (2019). It is also discussed in Lutz et al. (2013) who consider potential non-linearities. Using a regime-switching model, they distinguish two different pricing regimes - one applies during periods of high volatility and the other during periods of low volatility. By construction, the impact of explanatory variables on the allowance price can differ among the two regimes. In both regimes, they find the same set of relevant price drivers. Coal and gas prices, oil prices and the stock index are statistically significant determinants of the EUA price. In Regime 2, which is characterized by low and constant volatility, all significant price drivers show the anticipated sign. Regime 1 , however, shows a positive impact of the coal price. This goes against economic considerations that predict, as in the second regime, a negative effect of the coal price on allowance prices. These results gives further evidence that the relationship between the allowance price and its fundamentals might not be constant over time but be subject to (structural) changes.

The effect of the coal price on allowance prices causes further disagreement in findings. Similar to the results in the second regime of Lutz et al. (2013), Rickels et al. (2014) find a positive effect of the coal price on the allowance price in their single variable analysis. The paper by Rickels et al. (2014) differentiates itself 
from previous studies, because the authors investigate the effect of the choice of data series by performing various regressions with only one explanatory variable. They consider multiple data series for the different factors, from different sources and with different sampling frequency (daily and weekly). In their final regression specification, they do not include the coal price as a separate explanatory factor but as part of the switching price. Further evidence is found in Aatola et al. (2013) for the period 2005-2010 who find a negative coefficient of coal, while Hintermann (2010) finds it to be insignificant in Phase I data. In addition, Koch et al. (2014) look at the entire second Phase II and the first year of Phase III and find an insignificant coefficient of coal. However, the explicitly calculated fuel switching price is found to have a significant effect. It is obtained from gas and coal prices as well as the efficiency and emission rates of coal and gas plants in the EU ETS.

Regarding the effect of the gas price on allowance prices, there is no ambiguity. All studies find a positive and significant coefficient of the gas price independent which approach is used. In particular, in Hintermann (2010) it is the only explanatory variable with a significant effect throughout all considered specifications.

Overall, the regression models do not seem to provide a good fit to the data and many studies perform additional steps to improve the fit. Some look at sub-periods of their sample and run linear regressions separately for each period.

\subsubsection{The data}

We consider weekly data for the period from January 2008 to October 2018 resulting in $T=538$ observations. This sample period covers the entire Phase II and a large part of Phase III. We decide to exclude Phase I data because of its nature as a trial phase and because of the drop of allowance prices at the end of the phase which was due to the absence of banking possibilities.

As EUA price series, we use the December futures contract traded on the European Energy Exchange (EEX) as displayed in Figure 4.4. Most related papers rely on the December futures prices (Koch et al., 2014; Lutz et al., 2013; Aatola et al., 2013) since they are a frequently traded price series. As our main set of explanatory variables, we include natural gas, coal and oil prices as month-ahead futures. We further use two stock indices as supplementary indicators of current and expected economic activity. As a final explanatory variable we use European average temperature data.

The gas price is the settlement price of month-ahead Dutch TTF futures, denoted in EUR/MWh. TTF stand for Title Transfer Facility and is a virtual trading 


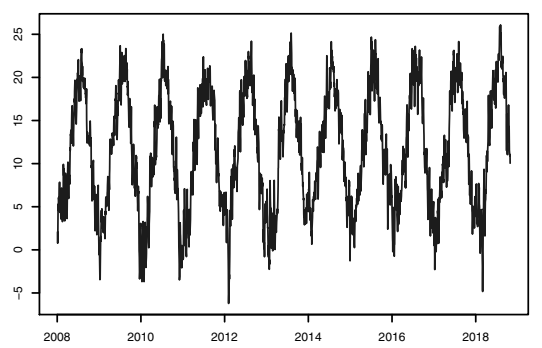

(a) Daily mean temperature

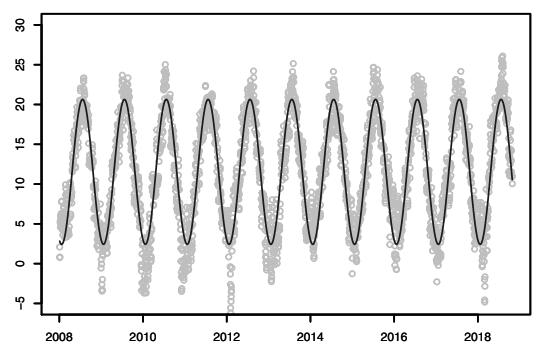

(b) Fourier fit

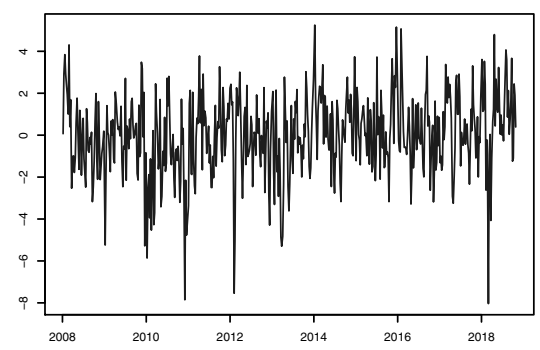

(c) Seasonally adjusted

Figure 4.5: Temperature data before and after the removal of the seasonal component.

point for natural gas in the Netherlands. The TTF futures contract is a frequently used series for gas prices in the related literature. Similarly, the coal price we consider is the settlement price of month-ahead futures based on the API2 index of the ARA region (Amsterdam-Rotterdam-Antwerp). The contract size is 1000 metric tonnes of thermal coal. ${ }^{6}$ Both are obtained from the EEX. For oil we rely on the historical futures prices (continuous contract) of Brent crude oil based on raw data from the Intercontinental Exchange (ICE), retrieved from Quandl. ${ }^{7}$ The contract size is 1,000 barrels. The coal and the oil prices need to be converted into EUR, as they are denoted in USD. This is done using the daily USD/EUR exchange rate data from Tullett Prebon. ${ }^{8}$ The first stock index is the STOXX Europe 50. As an alternative, we use data on a comparable index, which is some-

\footnotetext{
${ }^{6}$ To convert the coal price data into EUR/MWh, one simply has to divide the series by the conversion factor of 8.14. Since the conversion factor is constant and we consider first differences, this would not change our results.

${ }^{7}$ Retrieved from https://www.quandl.com/data/CHRIS/ICE_B1 on 21.01.2019

${ }^{8}$ Retrieved from https://quotes.wsj.com/fx/EURUSD/historical-prices on 21.01.2019
} 
times used in this context, the STOXX Europe 600 index. ${ }^{9}$ As temperature series we use temperature data for seven European cities from the European Climate Assessment \& Dataset (ECA\&D) which provides surface air temperature for 199 measurement stations in Europe. It is provided by The Royal Netherlands Meteorological Institute (KNMI). We refer to Klein Tank et al. (2002) for more details on the temperature series and measurement stations.

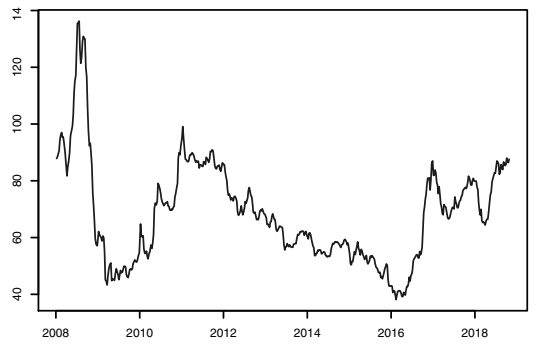

(a) Coal

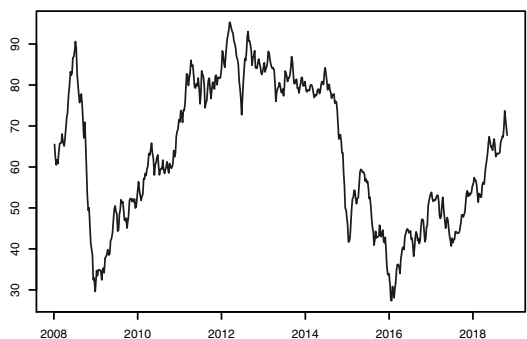

(c) Oil

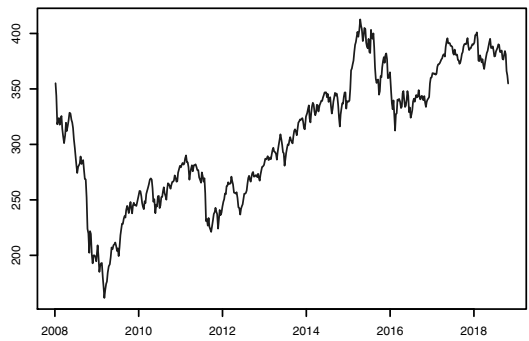

(e) Stoxx 600

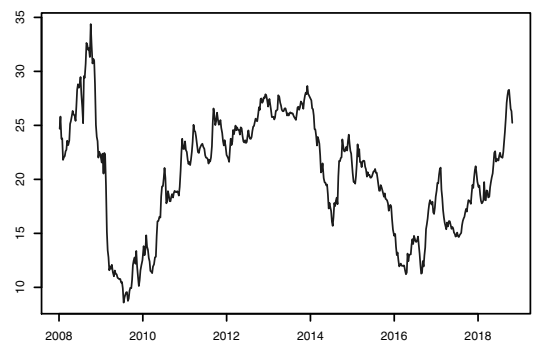

(b) Gas

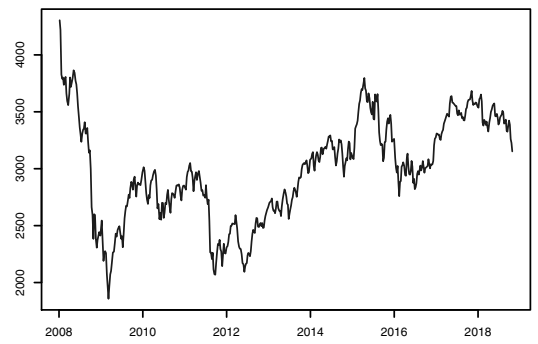

(d) Stoxx 50

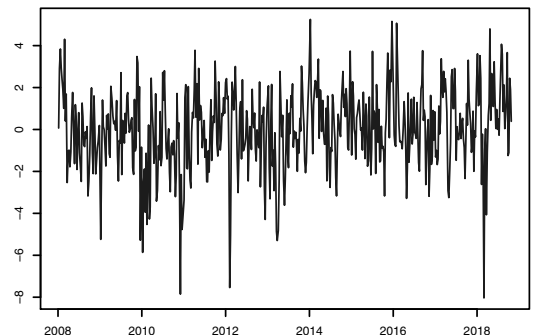

(f) Temperature averages

Figure 4.6: Plot of the time series in our data set.

\footnotetext{
${ }^{9}$ The stock index data are retrieved on 21.01.2019 from https://quotes.wsj.com/index/ XX/SXXP/historical-prices (STOXX Eur 600) and http://quotes.wsj.com/index/XX/SX5E/ historical-prices (STOXX Eur 50).
} 
We obtain daily mean temperature series for seven cities, located in seven different countries. They are spread out over Europe: Berlin, Budapest, De Bilt, Dublin, Lyon, Madrid and Stockholm. All series do not contain any missing observations and all were updated until the end of 2018. We take the average over all cities as our temperature series. It is displayed in Figure 4.5(a). We aggregate the data to weekly means in order to match our sample frequency. In addition, we remove seasonality by fitting a Fourier regression and subsequently, working with the residual series from this regression. This removes the seasonal component with the help of sine and cosine functions. More specifically, we fit the following regression

$$
\text { Temp }_{t}=\alpha_{1} \cos (2 \pi t)+\alpha_{2} \sin (2 \pi t)+\epsilon_{t}
$$

and continue to work with the residuals from this regression which are plotted in Figure $4.5(\mathrm{c})$. The remaining part (b) of Figure 4.5 plots the daily mean temperature (gray circles) together with the fitted Fourier terms (blue). We can see that the seasonal component is well captured by this method. ${ }^{10}$

Various other variables - e.g. data on renewable energy production, issued Certified Emission Reductions and water reservoir levels - appear in the literature. These variables are often found to be insignificant or to have a negligible effect in terms of the magnitude of the coefficients. Further limitations arise, since data on wind, solar or hydro production is only available for specific countries or regions. In our regression analysis we considered data on hydro power in Norway from the Norwegian Water Resources and Energy Directorate ${ }^{11}$ as well as wind and solar production data for Germany obtained from the database of the European Network of Transmission System Operators for Electricity (ENTSO-E). However, none of the time series showed a significant effect in our regression analysis and thus, we focus on the set of classical abatement related price drivers: coal and gas prices as well as oil prices, the stock indices and temperature data.

Figure 4.6 plots the various data series. All price and index series experienced a sharp drop at the beginning of our sample owing to the global financial crisis.

\footnotetext{
${ }^{10}$ As the method requires stationary data, removing the seasonality prior to the regression analysis is important to avoid spurious results. However, in this case part of the link between the temperature series and EUA prices might be lost. Therefore, it could be worthwhile to investigate ways to explicitly incorporate the seasonal component into the model or to construct an indicator for extremely cold periods. This is left for future work.

${ }^{11}$ Retrieved on 21.01.2019 from: http: //vannmagasinfylling.nve.no/Default . aspx?ViewType=AllYearsTable\&Omr=EL
} 
Additionally, panels (d) and (e) show that the two stock indices show an almost identical development over our sample period. A unit root test performed on the data shows that all series except for the temperature data contain a unit root. The results for the test are shown in Appendix 4.B. We therefore work with the series in log returns in the remainder of this application.

\subsubsection{Empirical results}

As a starting point and as a point of comparison for the nonparametric results, we show linear regression results for our dataset in Table 4.11 using Newey-West standard errors (Newey and West, 1987). The two significant factors are the gas and the oil price. The coal price, as an important driver, does not show a significant effect on the allowance price in this initial regression. This does not come as a surprise given the results from previous studies. Both significant coefficients are positive and thus show the anticipated sign. The coefficient of the coal price also shows the sign predicted by economic theory, while being insignificant. The results are robust regarding the choice of stock index; the estimates are very similar, if we include the alternative stock index, or if we include the oil price as the only indicator of economic activity. ${ }^{12}$

Before we present the results of our approach, we look for outliers in allowance price returns as the method is not designed to explain sudden jumps in the dependent variable. This is of particular relevance in this application since the previous literature suggests that there have been outliers present. We apply the impulse indicator saturation (IIS) approach proposed in Santos et al. (2008). This approach includes a dummy variable at every possible time point and performs expanding and contracting multiple block searches to determine which dummy variables should be retained. It is applied here to the EUA return series with a nominal significance level of $\alpha=0.005$.

The IIS method retains 10 dummy variables corresponding to 10 outliers located at observations 39, 50, 53, 167, 237, 246, 254, 257, 305 and 444. These observations are located at time points in October 2008, January and February 2009, June 2011, November 2012, January 2013, March and April 2013, March 2014 and December 2016. If we control for the impact of our most important explanatory variables - coal, gas, oil prices and temperature data - three of these

\footnotetext{
${ }^{12}$ Inclusion of the stock indices in addition the oil price left the other coefficients nearly unchanged and the index' coefficient is not significant. See Table 4.21 in Appendix 4.B for the results.
} 


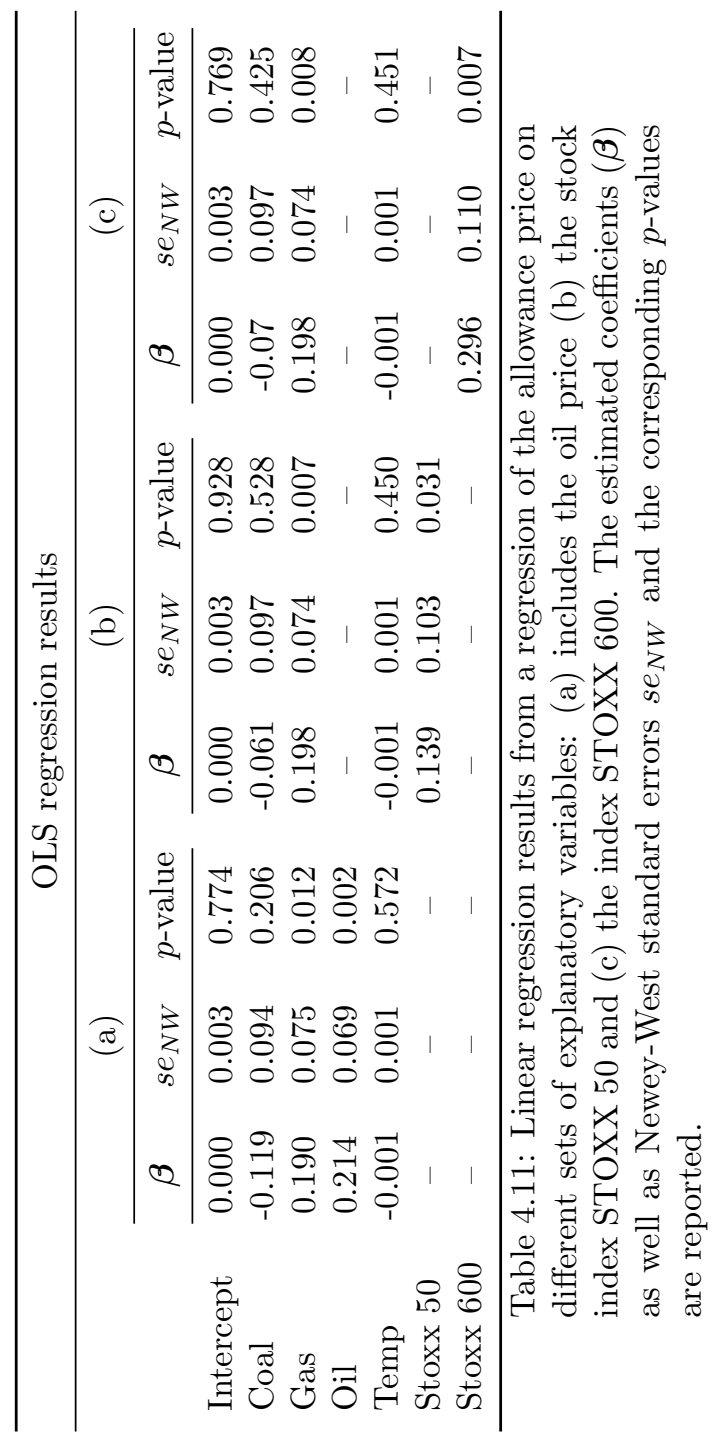




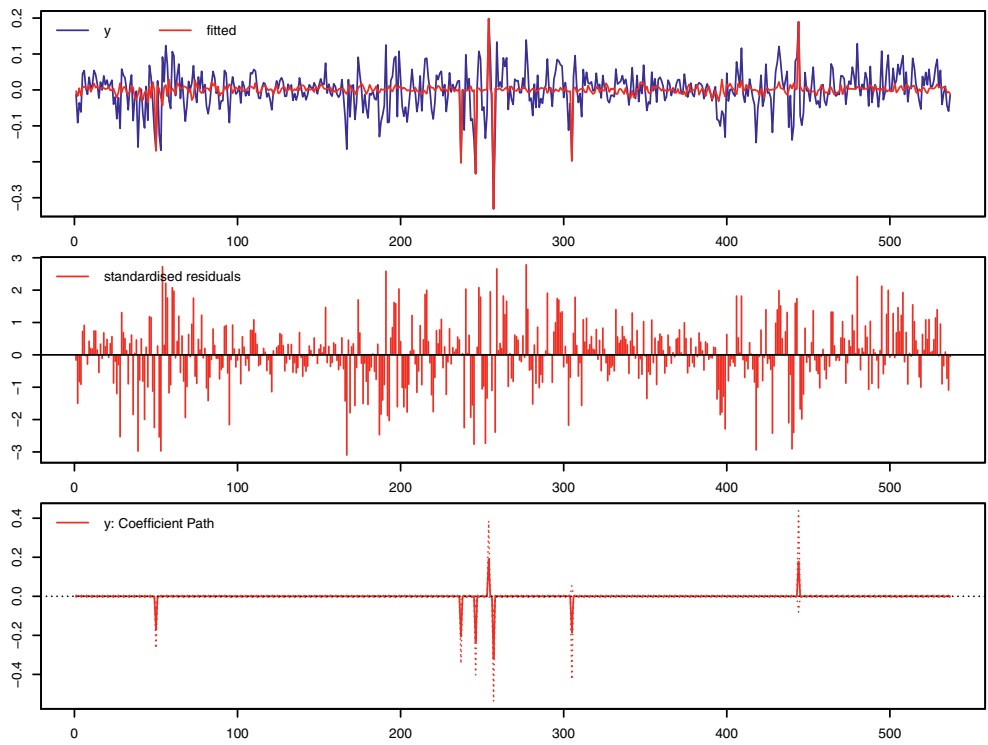

Figure 4.7: Summary of impulse indicator saturation results. Figure produced with $\mathrm{R}$ package gets. Top: EUA returns (blue) and fit of dummy regression (red); middle: standardized residuals after regression on dummies; bottom: retained dummy variables

outliers can be explained (at observation 39, 53 and 167). Figure 4.7 depicts the results of this IIS application. With this approach we retain 7 outliers which we delete from the EUA price series and subsequently perform our analysis. The results we present here are the results after outliers have been removed.

We apply the local linear estimator with the Epanechnikov kernel and a sieve wild bootstrap procedure with $B=999$ to generate $95 \%$ confidence bands. The results are plotted in Figure 4.9. In Appendix 4.B we repeat the analysis using the original data including all identified outliers. Figure 4.14 summarizes the results. Comparison of the two figures shows that there are no major differences. A noteworthy difference is that the confidence intervals get more narrow over some periods. In particular, this effect is visible for the gas, coal and oil price coefficient in the period from end 2012 to the beginning of 2014, where for the original data the confidence intervals experienced a widening which disappears with the removal of outliers. This does not change the results in a substantial way nor does it affect the conclusions drawn regarding the significance of coefficients.

To determine a suitable bandwidth, we perform data-driven bandwidth selection with the methods introduced in Section 4.3.2. We apply ordinary Cross 
Validation, Generalized Cross Validation, the Akaike-based criterion and Leave$(2 l+1)$-out Cross Validation with $l=4$. Table 4.12 presents the chosen bandwidths as well as their average. Figure 4.8 plots the corresponding selection criteria as functions of the bandwidth.

\begin{tabular}{|c|c|c|}
\hline \multicolumn{3}{|c|}{ Bandwidth selection } \\
\hline Method & $h_{\text {opt }}$ & Reference \\
\hline $\mathrm{CV}$ & 0.0874 & e.g. Fan and Gijbels (1996) \\
\hline $\mathrm{GCV}$ & 0.0800 & Zhou and $\mathrm{Wu}(2010)$ \\
\hline $\mathrm{AIC}$ & 0.0866 & Cai (2007) \\
\hline $\mathrm{L} 4 \mathrm{O} \mathrm{CV}$ & 0.0894 & Chu and Marron (1991) \\
\hline Average & 0.0859 & \\
\hline
\end{tabular}

Table 4.12: Optimal bandwidth chosen by different methods

We decide to use a bandwidth close to the average over the selected values, $h=0.09$. Given our simulation results, we repeat the analysis with a smaller value ( $h=0.05$ ) for robustness and observe that the main conclusions stay unchanged. In Appendix 4.B, we present the estimated parameter curves in Figure 4.15.

What we can observe in Figure 4.9 is that all of the estimated coefficients show periods of insignificance. In addition, we see significant time variation. We now go through all the results in detail. In Panel (a), the nonparametric trend fluctuates around zero for most of the considered time span. However, at the end of the sample, it turns significantly positive. This shows that the drastic price increase, visible in Figure 4.4, seems to be picked up by the trend component. Panel (b) shows that the coal price has a significant negative effect for two periods: one ranging from 2010 to mid 2011 and one from 2013 to mid 2015. Subsequently, the coefficient becomes positive and significant for a short period in $2016 / 2017$. This is a very interesting finding given that its coefficient was found to be insignificant in the linear regression analysis. This suggests that the time variation might have caused the insignificance in the linear regression results. Moving on to Panel (c), we see the coefficient of the gas price series. It has the expected positive sign and is significant in two periods. This is in line with previous findings as well as the linear regression results. However, apart from the boundary regions, we also find a period of insignificance in the usually stable gas price coefficient which is located in 2014. The coefficient of the oil price, as displayed in Panel (d) is positive and significant over two periods - until 2009 and from 2015 onward. Finally, from 


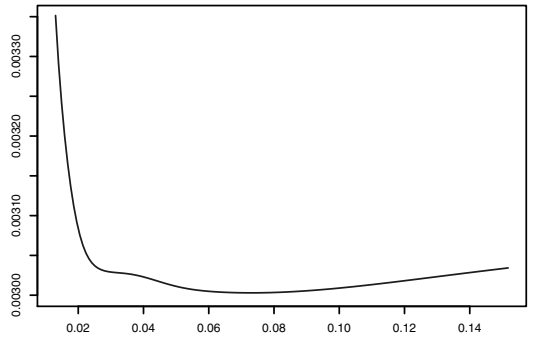

(a) $\mathrm{CV}$

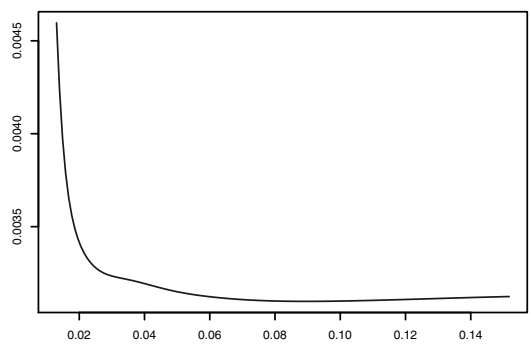

(c) Leave-9-out CV

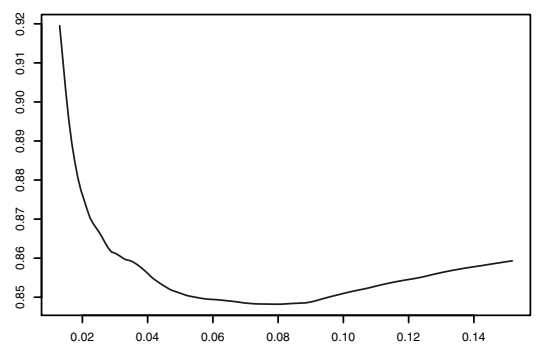

(b) GCV

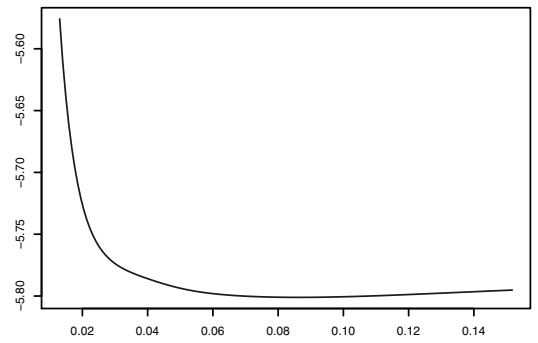

(d) AIC

Figure 4.8: The four different data-driven bandwidth selection criteria: (a) Cross validation, (b) Generalized cross validation, (c) Leave- $(2 l+1)$-out CV $(l=4),(d)$ Akaike-based bandwidth selection criterion

Panel (e) we see that the temperature series shows only two very short periods of significance and the magnitude of the coefficient estimate is, as in the linear regression results, negligible.

As an alternative indicator for economic activity, we also use the two stock indices as explanatory variables. We plot in Figure 4.10 the estimated parameter. The indices were entered separately into the nonparametric regression, replacing the oil price. We give here only the coefficient of the replaced data series and not the entire set of regressors. Switching from the oil price to either one of the stock indices does not change the parameter estimates for the remaining regressors. Their coefficient curves are almost identical to Figure 4.9. For the sake of brevity we do not plot them again. They can be obtained from the authors upon request.

Figure 4.10 reveals that the two stock indices produce very similar coefficient estimates. Given the shape of the two series (cf. Figure 4.6) this does not come as a surprise, as both indices show a comparable development over our sample period. Comparing the shape of the two curves in Figure 4.10 with the oil price 


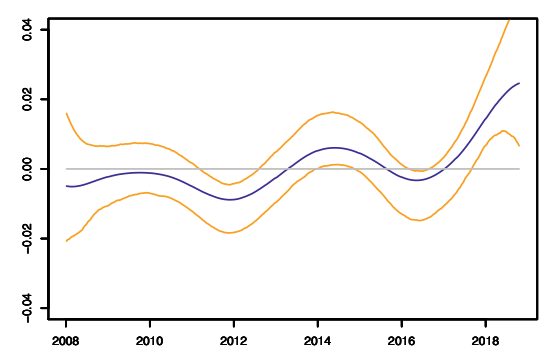

(a) $\hat{\beta}_{0}(t)$

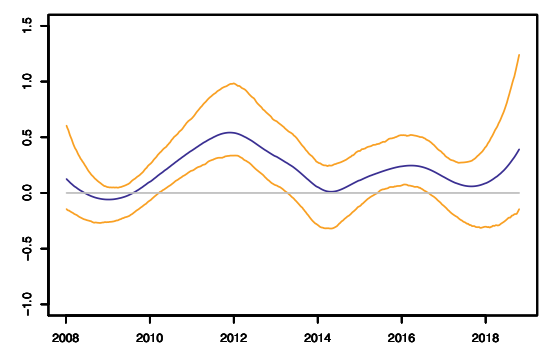

(c) $\hat{\beta}_{\text {gas }}(t)$

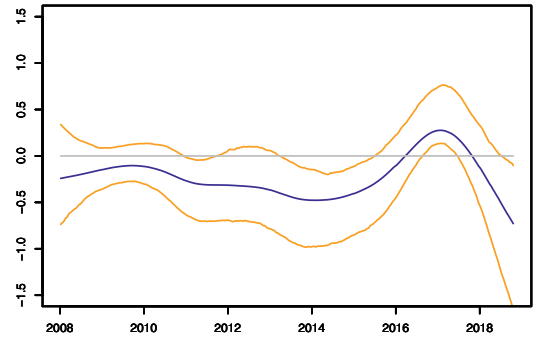

(b) $\hat{\beta}_{\text {coal }}(t)$

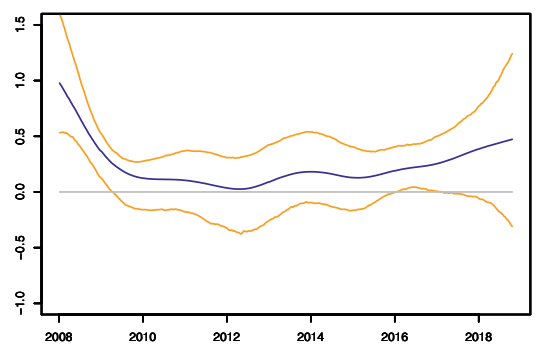

(d) $\hat{\beta}_{\text {oil }}(t)$

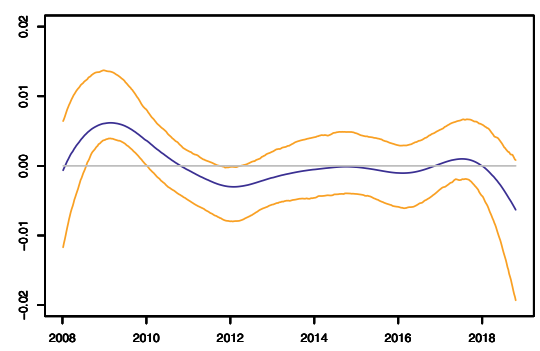

(e) $\hat{\beta}_{\text {temp }}(t)$

Figure 4.9: Nonparametrically estimated coefficient curves and 95\% confidence intervals

coefficient plotted in Figure 4.9(d) shows that there are also some similarities. All three parameter estimates are positive and show some periods of significance which are longest for the oil price coefficient. While the oil price coefficient becomes significant at the end of 2014 and stays significant until the end of the sample (disregarding the boundary effect), the stock indices have a significant coefficient only from the end of 2014 to the beginning of 2015 and the STOXX 600 index in 
2012 for a very short period. The period of significance is therefore overall shorter than for the oil price.

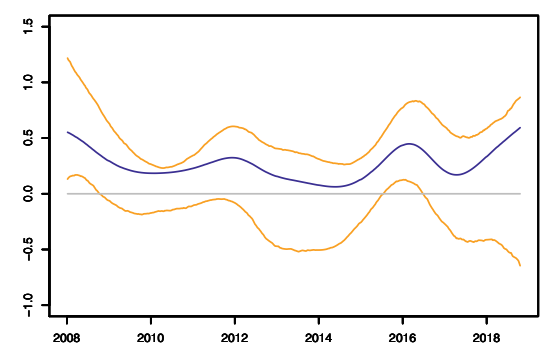

(a) $\hat{\beta}_{\text {Stoxx } 50}(t)$

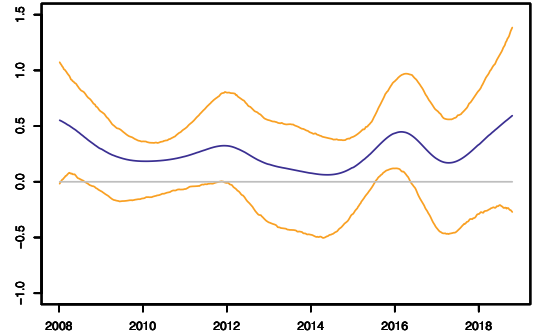

(b) $\hat{\beta}_{\text {Stoxx } 600}(t)$

Figure 4.10: Nonparametrically estimated coefficient curves and $95 \%$ confidence intervals for the two stock indices

As mentioned above, we add two additional explanatory variables to our nonparametric regression: energy supply from hydro power in Norway as well as data on electricity generation from wind for Germany. Both variables should have a negative effect on allowance prices, as generation from renewable energy sources reduces emissions and therefore the demand for allowances. The hydro power data are weekly data which contain a strong seasonal component which is, as for the temperature data, removed with the help of Fourier terms. The wind generation data is only available until the end of May 2018 which reduces our sample size for this regression exercise compared to the previous analysis. The sample comprises now 517 instead of 538 weekly observations. Added to the nonparametric regression, both new regressors have a coefficient estimate which is extremely low in magnitude for the whole sample. Both estimated coefficient curves are plotted in Figure 4.11. From Panel (a) we see that hydro power is significant over a very brief period in 2016. Panel (b) shows a period of significance for wind at the beginning of the sample until 2010. Although this period is quite long, given the small magnitude of the coefficient (in the order of $10^{-5}$ ), we consider this effect as negligible.

As a final supplementary analysis, we calculate the fuel switching price for the switch from coal to gas for electricity generation. The switching price is often included as an explanatory variable in the related literature (cf. Koch et al. (2014), Rickels et al. (2014)). It can be obtained from the coal and gas price series together with some additional values: the efficiency of coal and gas plants as well as the 


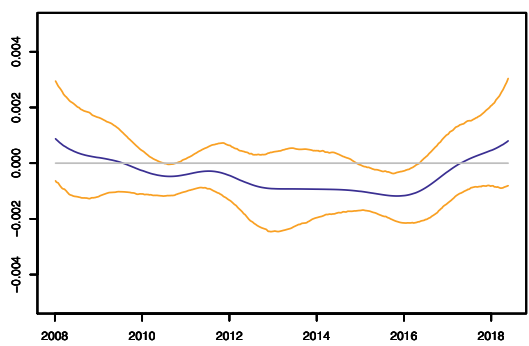

(a) $\hat{\beta}_{\text {Hydro }}(t)$

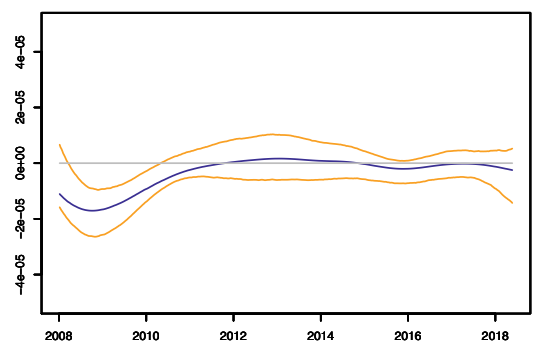

(b) $\hat{\beta}_{W i n d}(t)$

Figure 4.11: Nonparametrically estimated coefficient curves and 95\% confidence intervals for wind and hydro power generation

respective GHG emission factors. The emission factors are obtained from Juhrich (2016). The switching price is defined as follows

$$
\text { switch }_{t}=\frac{\eta_{\text {coal }} p_{\text {gas }}-\eta_{\text {gas }} p_{\text {coal }}}{\eta_{\text {gas }} f_{\text {coal }}-\eta_{\text {coal }} f_{\text {gas }}},
$$

where $\eta_{i}, f_{i}$ and $p_{i}$ are the plant efficiency, emission factor and fuel prices for $i=$ coal,gas, respectively. For details on this we refer to the review by Delarue et al. (2008). Figure 4.12 plots the resulting switching price as well as the estimated coefficient curve when replacing the gas and coal price series by the switching price. The switching price displayed in Panel (a) is obtained using our coal and gas price

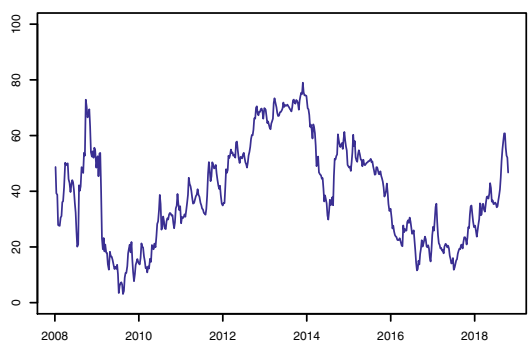

(a) Switching price

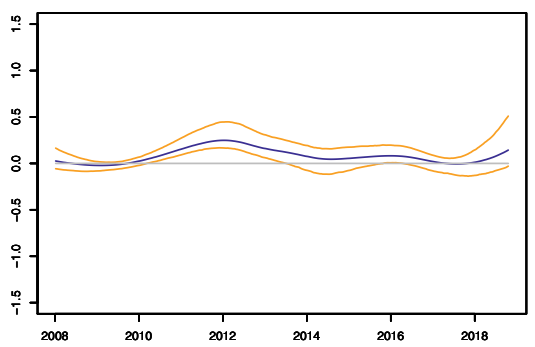

(b) $\hat{\beta}_{\text {Switch }}(t)$

Figure 4.12: Fuel switching price (Panel (a)) and nonparametrically estimated coefficient curves and $95 \%$ confidence intervals for the fuel switching price (Panel (b))

data as well as $\eta_{\text {gas }}=0.47, \eta_{\text {coal }}=0.36, f_{\text {gas }}=0.202$ and $f_{\text {coal }}=0.338$. Note 
that the gas and the coal price must both be denoted in EUR/MWh. This is why we need to divide our coal price by the conversion factor 8.14 . The general development of the switching price is similar to the gas price as plotted in Figure $4.6(\mathrm{~b})$.

From (4.22) we can see that the switching price is a linear combination of the gas and the coal price. Hence, we include it in place of the two price series in our regression. The result is presented in Panel (b) of Figure 4.12. The shape of the estimated coefficient curve closely resembles the coefficient of the gas price which is not surprising given the stronger effect of the gas price on allowance prices compared to the coal price. The estimated coefficient is, however, smaller and the second period of significance vanishes. Including the gas and the coal price separately and not as a linear combination is less restrictive and we therefore do not consider the switching price in our main model.

\subsection{Conclusion}

In this chapter, we propose a sieve bootstrap method to construct confidence intervals around nonparametrically estimated time-varying coefficient curves. The model allows for a flexible specification consisting of an intercept and multiple regressors, each with smooth functions of time as their coefficients. A major advantage of this model is that no functional form has to be specified in advance for any of these functions. Therefore, it is particularly suitable to model (economic) relationships which are suspected to be subject to gradual structural change.

We show in our extensive simulation study that the sieve bootstrap method provides confidence intervals with accurate coverage. This is the case for parameter curves of different shapes and even for small sample sizes and in the presence of serial correlation. We additionally propose a procedure to convert these confidence intervals into simultaneous confidence bands. Such bands are more useful than pointwise confidence intervals in such time-varying models since they allow for comparisons over time. They can be used, for instance, to verify whether the impact of a specific regressor has been constant over time - a question which cannot be answered using pointwise confidence intervals. Our simulation study reveals that the sieve bootstrap performs well in combination with the proposed procedure. The resulting simultaneous confidence bands display accurate coverage for almost all considered cases. When the simulated parameter curve is of a relatively simple 
shape, the method performs well for all considered values of the most important parameter (the bandwidth).

When the simulated parameter is more complex, the sensitivity to the bandwidth parameter increases as the coverage drops for larger bandwidth values. Given that this parameter is responsible for the smoothness of the estimated curve, this is not surprising since more complex shapes require an estimator which is able to adapt to more movements in the underlying curve. Investigating the performance of data-driven bandwidth selection methods, however, showed that on average larger values are selected. As the shape of the true parameter curve is unknown in practice, such methods have to be used with care. We recommend to additionally use a smaller bandwidth value than the selected one and to see if the results still hold when this bandwidth is applied. Extending the bootstrap method with a wild component has not proven to yield satisfactory results in our model set-up. The resulting confidence intervals are very narrow and display significantly lower coverage in our simulation study.

In the empirical application, we demonstrate the added value of our approach to a current topic in environmental economics: We study the price development of allowances in the largest cap and trade market for CO2 emissions - the European Emissions Trading Scheme. We provide evidence of time variation in the relationship between allowance prices in the EU ETS and their abatement-related fundamental price drivers. It supports previous research which has concluded that the relationship might be unstable. Due to the various periods of insignificance, our results offer a potential explanation for insignificant coefficients found with linear regression techniques used in some of the previous work. In addition, although the method offers great flexibility in modeling the relationship, the recent upward trend stays in the trend component indicating that this much discussed development is not driven by the included variables. This raises the question about the cause of the price increase which is still unanswered and provides an interesting starting point for further research in this area.

\section{A Additional simulation results}

We show here additional results for our main simulation study from Section 4.5. In addition, we present a second DGP which is inspired by time-varying CAPM models. For this DGP, we look at various $\operatorname{ARMA}(1,1)$ error specifications and several different bandwidths and study the performance of the sieve bootstrap. 


\section{A.1 Additions to the main simulation study}

In this section we provide additional results for the DGP presented in the main text. We divide the section into three sub-parts. First, we show results from a combination of the two error structures we considered in the main study: $\operatorname{ARMA}(1,1)$ $\operatorname{GARCH}(1,1)$. Second, we exclude the boundary points and third, we increase the sample size to 300 .

Table 4.13 displays results from the ARMA-GARCH specification. It combines both error structures we considered by adding a GARCH component to the ARMA errors. We look at three different ARMA specification with a GARCH error generated as in (4.20) with $\left(\alpha_{1}, \alpha_{2}\right)=(0.2,0.7)$. We investigate $\operatorname{AR}(1)$ errors with $\phi=0.1,0.3$ and $\mathrm{MA}(1)$ errors with $\psi=0.3$. The Table is split into four blocks: one for pointwise confidence intervals and three for simultaneous confidence bands over $G, G_{s u b}$ and the whole sample, respectively. In each block, the first pair of columns shows results for $\beta_{1}$ and the second pair of $\beta_{2}$. Each pair of

columns compares the sieve bootstrap (SB) with the sieve wild bootstrap (SWB). The rows present the coverage probabilities for the different ARMA specifications. The number in brackets below each coverage probability is the average median length of the intervals.

The results are comparable to the results from the pure GARCH specification. The sieve bootstrap outperforms the sieve wild counterpart. The difference is particularly high for simultaneity over the whole sample. The SB provides accurate pointwise coverage and simultanoeus coverage probabilities are quite high (around $90-92 \%$ for $G_{\text {sub }}, 89-91 \%$ for $G$ and $81-85 \%$ for the whole sample). The SWB results are considerably lower (88-90\% pointwise coverage, $75-86 \%$ for $G_{\text {sub }}, 68$ $83 \%$ for $G$ and $32-45 \%$ for the whole sample). A potential cause could be the repeatedly smaller interval width. This concludes the first part of this section.

As noted in Remark 4.1, the edge effects of the kernel estimator could distort the sieve bootstrap errors if the boundary residuals are resampled. Therefore, we exclude the boundary residuals and resample only from center residuals. We choose $\delta$ as in Remark 4.1 to be equal to the bandwidth. We make this choice because it is the truncation of the kernel estimator which is mainly responsible for the boundary effects and $n h$ observations get truncated. In Table 4.14 we report results for a small number of ARMA specification from our simulation study. Comparing these new results to the previous results we see at most a small improvement in coverage probabilities. The results do not seem to be greatly affected. The sieve bootstrap 
performance lies quite close to nominal level independent of whether or not we exclude boundary residuals.

The sieve wild bootstrap performance is more problematic. However, as no resampling is involved the boundary residuals stay at the boundary and we do not have to adapt the bootstrap algorithm. We would nevertheless like to investigate the effect of potential edge effects. Therefore, we repeat in Table 4.15 a limited number of specifications. For the calculations of the coverage probabilities, we simply exclude the first and last $(h * n)$ observations to rule out potential boundary effects - such as truncation of the kernel estimator - as cause for the poor performance of the sieve wild bootstrap in some cases. We look at three specifications for a bandwidth of $h=0.04$ : white noise errors, ARMA errors with $(\phi, \psi)=(0.3,0.3)$ as well as GARCH errors with $\left(\alpha_{1}, \alpha_{2}\right)=(0.2,0.7)$. Since the boundary points are excluded in the calculation of the simultaneous confidence bands over the sets $G$ and $G_{s u b}$, we only report results for pointwise coverage and simultaneity over the center part of the sample (i.e. the whole sample minus the boundary points).

Comparing the results for pointwise intervals with the previous values from Tables 4.1 and 4.6, we see a small improvement in coverage probabilities. Including the boundary regions, the SWB coverage probabilities for $\beta_{1}$ and $\beta_{2}$ in the white noise case were 90 and $91 \%$, respectively. Excluding the boundary points they are at $92 \%$. For the ARMA errors they were 85 and $86 \%$ and increased to 87 and $88 \%$. A larger improvement can be seen for simultaneous coverage which was as low as 40 and $48 \%$ for white noise or 24 and $30 \%$ for the ARMA errors (see Table 4.4). Now, the values are 58 and $66 \%$ for white noise as well as 41 and $48 \%$ for the ARMA errors. The SB produced better simultaneous results at 92 and $93 \%$ for white noise and 89 and $91 \%$ for ARMA. Excluding the boundary points, there is a small improvement of around 2 percentage points. In the GARCH case, the results show a similar pattern.

Overall, this exercise shows that excluding the boundary points can lead to some improvements but it is not the single explanation for why the sieve wild bootstrap performs so poorly, in particular, at simultaneous coverage. This concludes the second part of this section.

In Tables 4.16 and 4.17, we see how the coverage and lengths of the confidence intervals change when we increase the sample size from 100 to 300 . The first pair of columns of each table repeats selected results for $n=100$ from Tables 4.1 to 4.4. The second pair of columns shows the results for the larger sample size. The rows present a selected number for ARMA specifications. Here, we restrict ourselves 
to three cases: white noise, an $\mathrm{AR}(1)$ with coefficient of 0.3 and an MA(1) with coefficient of 0.3 . In each block, we first give results for $\beta_{1}$ and then for $\beta_{2}$. In this simulation exercise, we consider only one bandwidth $(h=0.04)$.

As expected, the coverage increases while the average median length becomes significantly smaller. In particular, for the sieve wild bootstrap, where coverage was below the nominal level for $n=100$, there is a visible improvement of coverage probabilities towards the nominal level. Pointwise confidence intervals now provide accurate coverage. For the simultaneous bands coverage still lies below the nominal level. For $\beta_{1}$, coverage probabilities are around $85 \%$ for $G$ and $88 \%$ for $G_{s u b}$. For $\beta_{2}$, the values are around $92 \%$ for $G$ and close to $95 \%$ for $G_{s u b}$. The white noise case always shows slightly better performance compared to the $\mathrm{AR}(1)$ and $\mathrm{MA}(1)$ cases. Simultaneity over the whole sample remains rather low at around $55 \%$ for $\beta_{1}$ and $64 \%$ for $\beta_{2}$.

The sieve bootstrap performs well in almost all cases. Coverage probabilities are even slightly too high in some cases. Merely the simultaneity over the whole sample remains at a small loss of coverage compared to the nominal level. While the $\mathrm{AR}(1)$ results are accurate, the white noise and $\mathrm{MA}(1)$ cases produce coverage of around $92-93 \%$ for $\beta_{1}$. In the white noise case, we even observe a small reduction of coverage. For $\beta_{2}$, only the MA(1) case lies below the nominal level with $93 \%$. Nevertheless, we conjecture that the performance is acceptable given the major reduction in the interval width.

\section{A.2 Time-varying CAPM models}

Of particular interest in financial econometrics are time-varying-beta CAPM models. To obtain evidence from an additional simulation study, we use a smooth transition model to simulate a CAPM model with time-varying beta coefficient.

Consider the following smooth transition model with three shifts:

$$
y_{t}=\beta_{1} x_{t}+\beta_{2} x_{t} G\left(t / n, \gamma_{1}, c_{1}\right)+\beta_{3} x_{t} G\left(t / n, \gamma_{2}, c_{2}\right)+\beta_{4} x_{t} G\left(t / n, \gamma_{3}, c_{3}\right)+u_{t}
$$

where $x_{t}=0.2 x_{t-1}+\xi_{t}$ with $\xi_{t} \sim N(0,1)$. The CAPM $\alpha$, which is the intercept of the above regression, is set to zero in all regimes. The function $G(\cdot, \cdot, \cdot)$ is a logistic transition function of the following form:

$$
G(x, \gamma, c)=(1+\exp \{-\gamma(x-c)\})^{-1}
$$


We induce different degrees of dependence with an $\operatorname{ARMA}(1,1)$ specification for the error term $\left\{u_{t}\right\}_{t=1}^{n}$

$$
u_{t}=\phi u_{t-1}+\psi \epsilon_{t-1}+\epsilon_{t} \quad \epsilon_{t} \sim N\left(0, \frac{1-\phi^{2}}{1+\psi^{2}+2 \phi \psi}\right)
$$

where the variance of $\epsilon_{t}$ is such that the signal to noise ratio does not change when we vary $\phi$ and $\psi$.

The fact that three shifts are used comes from an application to the banking sector as considered in Caporale (2012) who estimates a CAPM model with breaks using break point estimation as in Bai and Perron (1998). Caporale (2012) detects three breaks when estimating a CAPM model for returns of a banking stock index. All three breaks correspond to a specific event in the banking sector - like a US presidential regime shift or a crisis. He finds that the banking beta increases after the first two breaks and decreases after the third, while the CAPM intercept is not significantly different from zero in all periods. The latter result supports the theory behind the CAPM model and the behavior of the betas are in line with the nature of the different events.

We mimic the development in the banking sector by choosing the location parameters as well as the beta coefficients accordingly. The first shift occurs after $30 \%$ of the sample, the second after $60 \%$ and the final shift takes place towards the end of the sample at $90 \%$. The beta coefficient starts at $\beta_{1}=0.5$ and increases to $\beta_{2}=0.9$ and $\beta_{3}=1.4$ until it goes back to its initial level at $\beta_{4}=0.5$. We let $\gamma_{1}=10, \gamma_{2}=3, \gamma_{3}=7$.

This specification gives us two objects to estimate - the intercept $\alpha$, which in this case is a straight line at zero, and the coefficient curve $\beta$, a curve with one peak. We plot $\beta$ in Figure 4.13 together with estimated coefficient curves using two different bandwidths, $h=0.04$ and $h=0.08$.

Sieve bootstrap results are presented in Tables 4.18 and 4.19. The four different blocks in each table, report results for four different bandwidths: 0.02, 0.04, 0.06 and 0.08 . In each block, four columns first present pointwise results and subsequently, results of the simultaneous confidence bands for the sets $G$ and $G_{s u b}$. We look at nine different $\operatorname{ARMA}(1,1)$ specifications in the different rows of each block. We present coverage probabilities as well as the average median lengths in brackets below each coverage probability. Table 4.18 reports results for the intercept $\alpha$ and Table 4.19 for the coefficient curve $\beta$. 


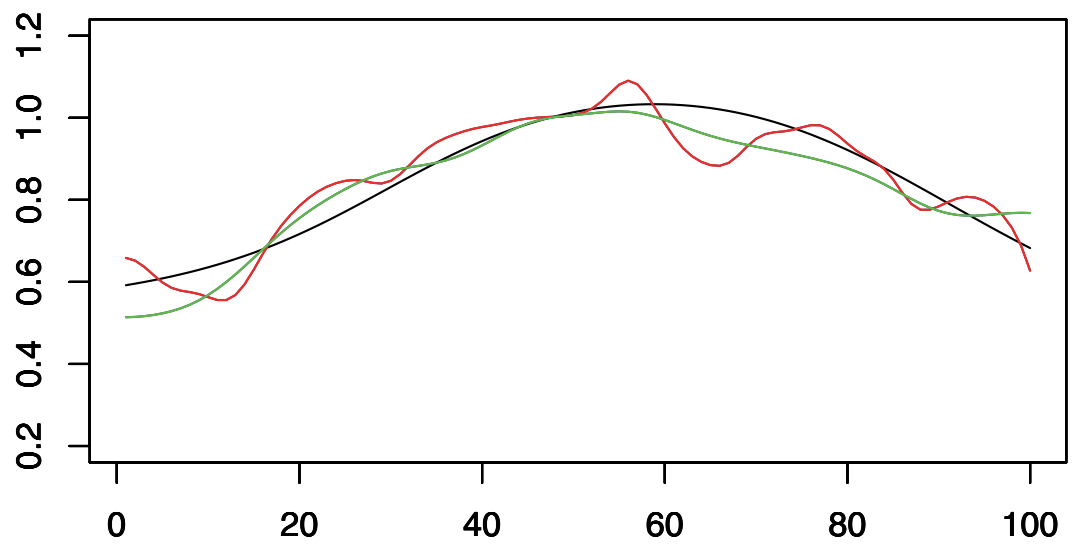

Figure 4.13: Plot of the $\beta$ coefficient as well as two estimated coefficient curves using $h=0.04$ (red) and $h=0.08$ (green) generated with $\operatorname{AR}(1)(\phi=0.1)$ errors.

Pointwise coverage probabilities are close to the nominal level in all considered specifications. Even strong serial correlation, for instance with $(\phi, \psi)=(0.5,0.5)$, are handled well by the sieve bootstrap. For the simultaneous confidence bands, coverage for $\alpha$ drops to around $81-94 \%$ for $G_{\text {sub }}$ and to $76-94 \%$ for $G$. The differences arise since negative correlation can be handled better than positive correlation. The lowest values are achieved for cases with strong positive correlation.

The results are quite robust regarding the choice of bandwidth (comparing the different blocks in each table). For the low positive and the negative correlation cases, larger bandwidths produce slightly better results, while for strong positive correlation, smaller bandwidths seemed to be preferred. This does not hold for simultaneity over the whole sample where the larger bandwidths produce the best results. The differences are overall not very pronounced. Comparing the two tables, we see that results for $\beta$ are better than for the constant parameter $\alpha$. The coverage is very close to the nominal level in almost all cases.

\section{B Additional empirical results}

Table 4.20 presents the results of the unit root tests. In addition to the Augmented Dickey-Fuller (ADF) test (Dickey and Fuller, 1979), we also use the tests by Phillips and Perron (1988), Kwiatkowski et al. (1992) and Leybourne et al. 
(1998). The test statistics are given for all series in levels $\left(y_{t}\right)$ as well as the log return data, which is defined as the first difference of the natural logarithm $\left(r_{y_{t}}=\ln \left(y_{t} / y_{t-1}\right)\right)$. The bottom part of the table shows the critical values for significance levels of $1 \%, 5 \%$ and $10 \%$. Apart from the KPSS test, all tests are left-sided, and hence, we reject the null hypothesis, if the test statistic is smaller than the critical value.

According to the ADF test, the unit root null hypothesis cannot be rejected at a $1 \%$ significance level in all cases but the temperature data. This holds true for the data in levels $\left(y_{t}\right)$. The only series for which a unit root would be rejected in favor of stationarity are the two stock indices at a $5 \%$ and $10 \%$ level, respectively. The log return series $\left(r_{y_{t}}\right)$ are all stationary.

The Augmented-Dickey-Fuller test, which has mainly been applied in the previous literature, considers a fixed mean or a linear trend under the alternative. It has been shown that this can quite frequently lead to spurious rejections of the unit root null hypothesis. Working with data in first differences if the data are trend stationary can substantially change the results. Therefore, it is crucial to carefully select the alternative hypothesis of unit root tests.

To address this, we additionally apply the tests by Phillips and Perron (1988), Kwiatkowski et al. (1992) and Leybourne et al. (1998). The latter test is similar to the ADF test, but it considers a smoothly varying time trend under the alternative hypothesis. The trend can undergo one transition and the time point as well as speed of the transition is determined endogenously by the test. This modification allows for much more flexibility under the alternative which is an advantage given our complex data series.

The results of the three additional tests are also presented in Table 4.20. The Phillips-Perron (PP) test results are presented in the third and fourth column. They confirm the results from the ADF test with the exception being that the unit root null hypothesis is now rejected for the two stock indices at the $1 \%$ significance level. Results of the more flexible test by Leybourne et al. (1998) (LNV) is given in the next two columns. According to this test, all series but the temperature data contain a unit root. The KPSS test, which is presented in the last two columns, comes to the same conclusion. Here, the null hypothesis is (trend) stationarity and the alternative is a unit root process.

Combining the above results with the ADF results, we conclude that allowance prices as well as all fuel prices and stock indices contain a unit root, the temperature data are stationary. 
In addition, we see in Table 4.21 that the linear regression results do not improve when we add the two stock indices to the model. Both show insignificant coefficients which could be due to the fact that with the oil price we already have some kind of measure of economic activity. This is why we leave them out in the presentation of the main results.

In Figure 4.14 we repeat the nonparametric regression exercise given in the main text without first removing the outliers from the data. In some cases, the confidence intervals get temporarily wider compared to Figure 4.9 presented above. This holds in particular for the gas and the oil price coefficient around 2013/2014. However, the main conclusions drawn in this chapter do not change.

Finally, we see in Figure 4.15 that these conclusions remain unchanged when we use a smaller bandwidth than the one suggested by the data-driven bandwidth selection methods. Here, we select $h=0.05$ which produced more consistent results in our simulation study. As expected, the estimated coefficient curves show more variability, but the main results highlighted in the above analysis are still visible, for instance, the two periods of significance of the gas price as well as the short period of a positive coal price coefficient. 


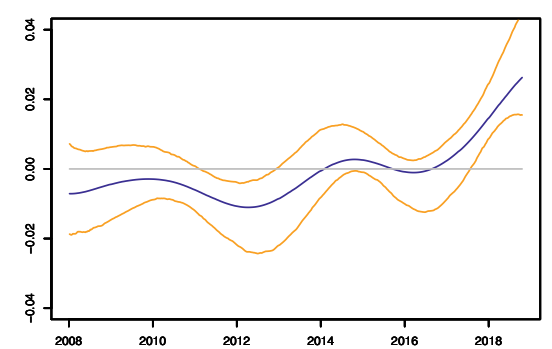

(a) $\hat{\beta}_{0}(t)$

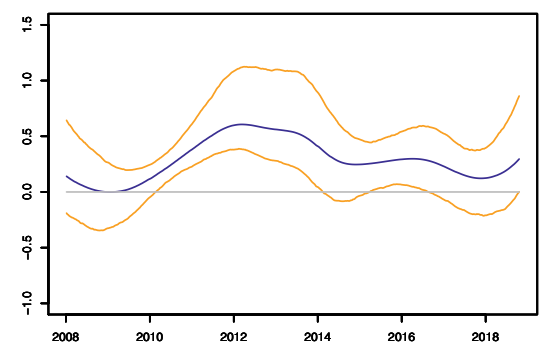

(c) $\hat{\beta}_{\text {gas }}(t)$

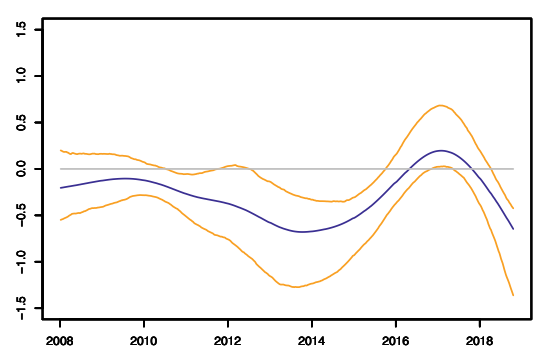

(b) $\hat{\beta}_{\text {coal }}(t)$

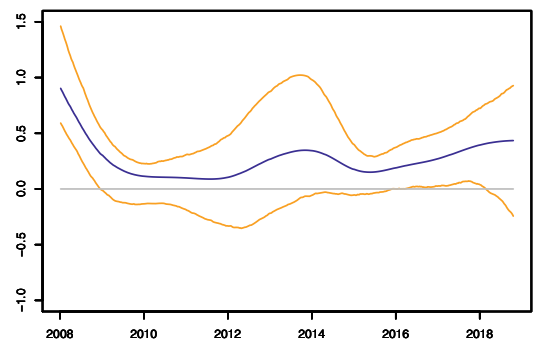

(d) $\hat{\beta}_{\text {oil }}(t)$

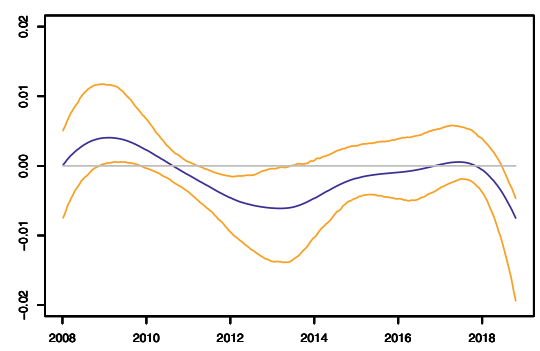

(e) $\hat{\beta}_{t e m p}(t)$

Figure 4.14: Nonparametrically estimated coefficient curves and 95\% confidence intervals before the removal of outliers 


\begin{tabular}{|c|c|c|c|c|c|}
\hline \multirow{7}{*}{ pw } & \multirow[b]{2}{*}{$(\phi, \psi)$} & \multicolumn{2}{|c|}{$\beta_{1}$} & \multicolumn{2}{|c|}{$\beta_{2}$} \\
\hline & & $\mathrm{SB}$ & SWB & SB & SWB \\
\hline & $(0.1,0)$ & $\begin{array}{c}0.949 \\
(0.308)\end{array}$ & $\begin{array}{c}0.891 \\
(0.274)\end{array}$ & $\begin{array}{c}0.956 \\
(0.305)\end{array}$ & $\begin{array}{c}0.903 \\
(0.253)\end{array}$ \\
\hline & \multirow{2}{*}{$(0.3,0)$} & 0.948 & 0.877 & 0.955 & 0.893 \\
\hline & & $(0.320)$ & $(0.274)$ & $(0.314)$ & $(0.255)$ \\
\hline & \multirow{2}{*}{$(0,0.3)$} & 0.948 & 0.878 & 0.955 & 0.894 \\
\hline & & $(0.319)$ & $(0.274)$ & $(0.313)$ & $(0.255)$ \\
\hline \multirow{8}{*}{$G_{\text {sub }}$} & \multirow[b]{2}{*}{$(\phi, \psi)$} & \multicolumn{2}{|c|}{$\beta_{1}$} & \multicolumn{2}{|c|}{$\beta_{2}$} \\
\hline & & $\mathrm{SB}$ & SWB & $\mathrm{SB}$ & SWB \\
\hline & $(010)$ & 0.911 & 0.780 & 0.920 & 0.855 \\
\hline & $(U .1, U)$ & $(0.404)$ & $(0.355)$ & $(0.400)$ & $(0.330)$ \\
\hline & $(030$ & 0.903 & 0.754 & 0.916 & 0.828 \\
\hline & $(0.3,0)$ & $(0.420)$ & $(0.356)$ & $(0.412)$ & $(0.333)$ \\
\hline & \multirow{2}{*}{$(0,0.3)$} & 0.903 & 0.757 & 0.908 & 0.835 \\
\hline & & $(0.419)$ & $(0.355)$ & $(0.411)$ & $(0.333)$ \\
\hline \multirow{8}{*}{$G$} & \multirow[b]{2}{*}{$(\phi, \psi)$} & \multicolumn{2}{|c|}{$\beta_{1}$} & \multicolumn{2}{|c|}{$\beta_{2}$} \\
\hline & & SB & SWB & $\mathrm{SB}$ & SWB \\
\hline & \multirow{2}{*}{$(0.1,0)$} & 0.893 & 0.726 & 0.912 & 0.829 \\
\hline & & $(0.437)$ & $(0.387)$ & $(0.433)$ & $(0.359)$ \\
\hline & \multirow{2}{*}{$(0.3,0)$} & 0.894 & 0.676 & 0.910 & 0.788 \\
\hline & & $(0.455)$ & $(0.388)$ & $(0.446)$ & $(0.362)$ \\
\hline & \multirow{2}{*}{$(0,0.3)$} & 0.893 & 0.695 & 0.911 & 0.793 \\
\hline & & $(0.454)$ & $(0.387)$ & $(0.445)$ & $(0.362)$ \\
\hline \multirow{8}{*}{$1, \cdots, n$} & \multirow[b]{2}{*}{$(\phi, \psi)$} & \multicolumn{2}{|c|}{$\beta_{1}$} & \multicolumn{2}{|c|}{$\beta_{2}$} \\
\hline & & SB & SWB & SB & SWB \\
\hline & \multirow{2}{*}{$(0.1,0)$} & 0.834 & 0.373 & 0.843 & 0.451 \\
\hline & & $(0.465)$ & $(0.411)$ & $(0.461)$ & $(0.381)$ \\
\hline & \multirow{2}{*}{$(0.3,0)$} & 0.813 & 0.337 & 0.846 & 0.397 \\
\hline & & $(0.484)$ & $(0.412)$ & $(0.474)$ & $(0.384)$ \\
\hline & \multirow{2}{*}{$(0,0.3)$} & 0.815 & 0.321 & 0.852 & 0.394 \\
\hline & & $(0.482)$ & $(0.411)$ & $(0.473)$ & $(0.384)$ \\
\hline
\end{tabular}

Table 4.13: $\overline{\operatorname{ARMA}(1,1)-\operatorname{GARCH}(1,1) \text { with varying }(\phi, \psi) \text { and }\left(\alpha_{1}, \alpha_{2}\right)}=(0.2,0.7)$ 


\begin{tabular}{|c|c|c|c|c|}
\hline & \multicolumn{2}{|c|}{ white noise } & \multicolumn{2}{|c|}{$\phi=0.1$} \\
\hline & $\beta_{1}$ & $\beta_{2}$ & $\beta_{1}$ & $\beta_{2}$ \\
\hline pw & $\begin{array}{c}0.956 \\
(0.307)\end{array}$ & $\begin{array}{c}0.956 \\
(0.306)\end{array}$ & $\begin{array}{c}0.954 \\
(0.306)\end{array}$ & $\begin{array}{c}0.957 \\
(0.305)\end{array}$ \\
\hline$G_{s u b}$ & $\begin{array}{c}0.951 \\
(0.401)\end{array}$ & $\begin{array}{c}0.944 \\
(0.399)\end{array}$ & $\begin{array}{c}0.934 \\
(0.400)\end{array}$ & $\begin{array}{c}0.937 \\
(0.2398)\end{array}$ \\
\hline$G$ & $\begin{array}{c}0.934 \\
(0.434)\end{array}$ & $\begin{array}{c}0.945 \\
(0.432)\end{array}$ & $\begin{array}{c}0.938 \\
(0.432)\end{array}$ & $\begin{array}{c}0.934 \\
(0.431)\end{array}$ \\
\hline \multirow[t]{3}{*}{$1, \cdots, n$} & $\begin{array}{c}0.906 \\
(0.460)\end{array}$ & $\begin{array}{c}0.916 \\
(0.459)\end{array}$ & $\begin{array}{c}0.898 \\
(0.459)\end{array}$ & $\begin{array}{c}0.898 \\
(0.457)\end{array}$ \\
\hline & \multicolumn{2}{|c|}{$\psi=0.3$} & \multicolumn{2}{|c|}{$(\phi, \psi)=(0.3,0.3)$} \\
\hline & $\beta_{1}$ & $\beta_{2}$ & $\beta_{1}$ & $\beta_{2}$ \\
\hline pw & $\begin{array}{c}0.956 \\
(0.299)\end{array}$ & $\begin{array}{c}0.957 \\
(0.293)\end{array}$ & $\begin{array}{c}0.951 \\
(0.360)\end{array}$ & $\begin{array}{c}0.953 \\
(0.349)\end{array}$ \\
\hline$G_{s u b}$ & $\begin{array}{c}0.943 \\
(0.390)\end{array}$ & $\begin{array}{c}0.948 \\
(0.383)\end{array}$ & $\begin{array}{c}0.934 \\
(0.470)\end{array}$ & $\begin{array}{c}0.933 \\
(0.456)\end{array}$ \\
\hline$G$ & $\begin{array}{c}0.934 \\
(0.421)\end{array}$ & $\begin{array}{c}0.943 \\
(0.413)\end{array}$ & $\begin{array}{c}0.923 \\
(0.509)\end{array}$ & $\begin{array}{c}0.926 \\
(0.493)\end{array}$ \\
\hline$\{1, \cdots, n\}$ & $\begin{array}{c}0.911 \\
(0.448)\end{array}$ & $\begin{array}{c}0.926 \\
(0.439)\end{array}$ & $\begin{array}{c}0.889 \\
(0.540)\end{array}$ & $\begin{array}{c}0.887 \\
(0.523)\end{array}$ \\
\hline
\end{tabular}

Table 4.14: Excluding the first and last $(h * n)$ residuals in Step 1 of the sieve bootstrap, with $h=0.04$

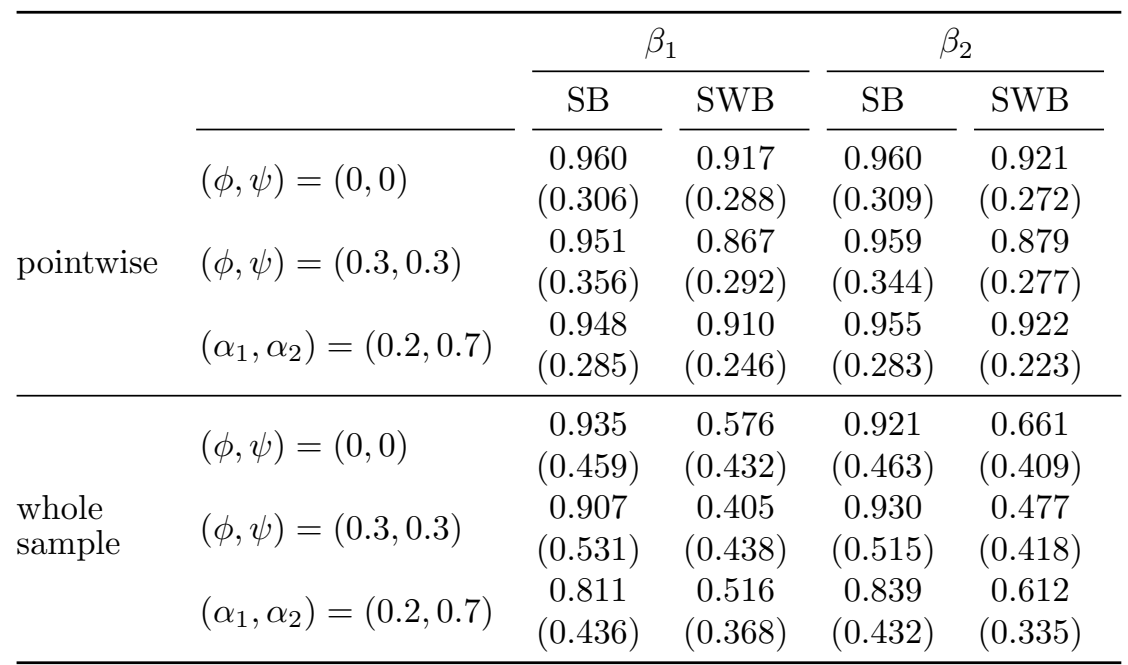

Table 4.15: Excluding the first and last $(h * n)$ observations when calculating coverage probabilities, with $h=0.04$ 


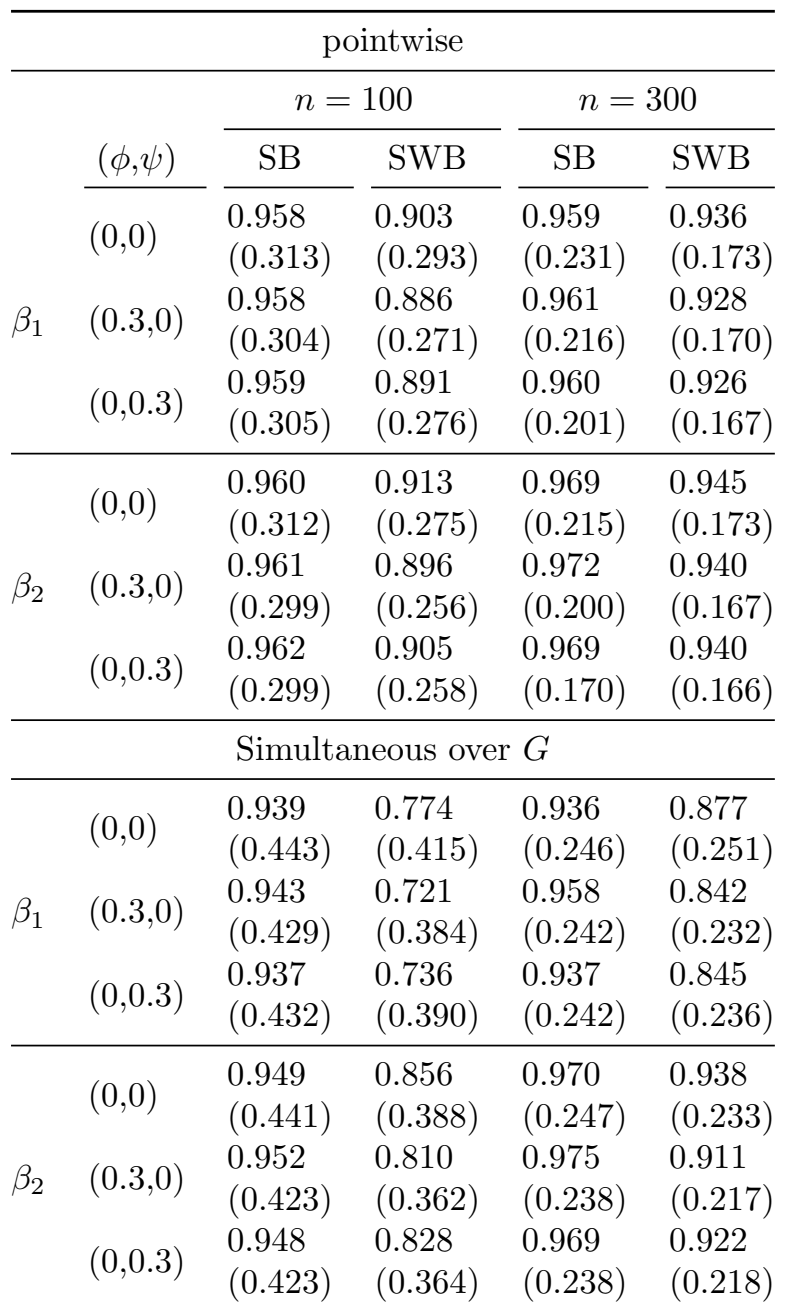

Table 4.16: Coverage probabilities for two sample sizes, $\operatorname{ARMA}(1,1), h=0.04$ 


\begin{tabular}{|c|c|c|c|c|c|}
\hline & \multicolumn{5}{|c|}{ Simultaneous over $G_{s u b}$} \\
\hline & \multirow[b]{2}{*}{$(\phi, \psi)$} & \multicolumn{2}{|c|}{$n=100$} & \multicolumn{2}{|c|}{$n=300$} \\
\hline & & SB & SWB & $\mathrm{SB}$ & SWB \\
\hline \multirow{3}{*}{$\beta_{1}$} & $(0,0)$ & $\begin{array}{l}0.940 \\
(0.408)\end{array}$ & $\begin{array}{l}0.810 \\
(0.381)\end{array}$ & $\begin{array}{l}0.930 \\
(0.227)\end{array}$ & $\begin{array}{l}0.893 \\
(0.231)\end{array}$ \\
\hline & $(0.3,0)$ & $\begin{array}{l}0.944 \\
(0.396)\end{array}$ & $\begin{array}{l}0.787 \\
(0.353)\end{array}$ & $\begin{array}{l}0.946 \\
(0.223)\end{array}$ & $\begin{array}{l}0.889 \\
(0.216)\end{array}$ \\
\hline & $(0,0.3)$ & $\begin{array}{l}0.934 \\
(0.398)\end{array}$ & $\begin{array}{l}0.796 \\
(0.358)\end{array}$ & $\begin{array}{l}0.940 \\
(0.223)\end{array}$ & $\begin{array}{l}0.880 \\
(0.216)\end{array}$ \\
\hline \multirow{3}{*}{$\beta_{2}$} & $(0,0)$ & $\begin{array}{l}0.956 \\
(0.407)\end{array}$ & $\begin{array}{l}0.893 \\
(0.358)\end{array}$ & $\begin{array}{l}0.977 \\
(0.228)\end{array}$ & $\begin{array}{l}0.947 \\
(0.214)\end{array}$ \\
\hline & $(0.3,0)$ & $\begin{array}{l}0.955 \\
(0.391)\end{array}$ & $\begin{array}{l}0.861 \\
(0.334)\end{array}$ & $\begin{array}{l}0.977 \\
(0.219)\end{array}$ & $\begin{array}{l}0.947 \\
(0.200)\end{array}$ \\
\hline & $(0,0.3)$ & $\begin{array}{l}0.959 \\
(0.390)\end{array}$ & $\begin{array}{l}0.890 \\
(0.336)\end{array}$ & $\begin{array}{l}0.969 \\
(0.219)\end{array}$ & $\begin{array}{l}0.943 \\
(0.201)\end{array}$ \\
\hline \multicolumn{6}{|c|}{ Simultaneous over whole sample } \\
\hline \multirow{3}{*}{$\beta_{1}$} & $(0,0)$ & $\begin{array}{l}0.921 \\
(0.470)\end{array}$ & $\begin{array}{l}0.397 \\
(0.441)\end{array}$ & $\begin{array}{l}0.915 \\
(0.263)\end{array}$ & $\begin{array}{l}0.581 \\
(0.269)\end{array}$ \\
\hline & $(0.3,0)$ & $\begin{array}{l}0.916 \\
(0.455)\end{array}$ & $\begin{array}{l}0.333 \\
(0.408)\end{array}$ & $\begin{array}{l}0.943 \\
(0.259)\end{array}$ & $\begin{array}{l}0.542 \\
(0.252)\end{array}$ \\
\hline & $(0,0.3)$ & $\begin{array}{l}0.913 \\
(0.458)\end{array}$ & $\begin{array}{l}0.360 \\
(0.415)\end{array}$ & $\begin{array}{l}0.927 \\
(0.259)\end{array}$ & $\begin{array}{l}0.541 \\
(0.253)\end{array}$ \\
\hline \multirow{3}{*}{$\beta_{2}$} & $(0,0)$ & $\begin{array}{l}0.929 \\
(0.468)\end{array}$ & $\begin{array}{l}0.483 \\
(0.413)\end{array}$ & $\begin{array}{l}0.958 \\
(0.264)\end{array}$ & $\begin{array}{l}0.666 \\
(0.249)\end{array}$ \\
\hline & $(0.3,0)$ & $\begin{array}{l}0.918 \\
(0.449)\end{array}$ & $\begin{array}{l}0.426 \\
(0.385)\end{array}$ & $\begin{array}{l}0.969 \\
(0.255)\end{array}$ & $\begin{array}{l}0.628 \\
(0.232)\end{array}$ \\
\hline & $(0,0.3)$ & $\begin{array}{l}0.933 \\
(0.449)\end{array}$ & $\begin{array}{l}0.451 \\
(0.387)\end{array}$ & $\begin{array}{l}0.927 \\
(0.212)\end{array}$ & $\begin{array}{l}0.622 \\
(0.253)\end{array}$ \\
\hline
\end{tabular}

Table 4.17: Coverage probabilities for two sample sizes (cont.), $\operatorname{ARMA}(1,1)$, $h=0.04$ 


\begin{tabular}{|c|c|c|c|c|c|c|}
\hline$(\phi, \psi)$ & $\mathrm{w}$ & $G_{s u b}$ & $G$ & $\mathrm{pw}$ & $G_{s u b}$ & $G$ \\
\hline & \multicolumn{3}{|c|}{$h=0.02$} & \multicolumn{3}{|c|}{$h=0.04$} \\
\hline$(0.1,0)$ & $\begin{array}{l}0.931 \\
(0.411)\end{array}$ & $\begin{array}{l}0.893 \\
(0.533)\end{array}$ & $\begin{array}{l}0.881 \\
(0.577)\end{array}$ & $\begin{array}{l}0.937 \\
(0.285)\end{array}$ & $\begin{array}{l}0.909 \\
(0.373)\end{array}$ & $\begin{array}{l}0.902 \\
(0.405)\end{array}$ \\
\hline$(0.3,0)$ & $\begin{array}{l}0.925 \\
(0.443)\end{array}$ & $\begin{array}{l}0.889 \\
(0.575)\end{array}$ & $\begin{array}{l}0.863 \\
(0.622)\end{array}$ & $\begin{array}{l}0.930 \\
(0.317)\end{array}$ & $\begin{array}{l}0.898 \\
(0.415)\end{array}$ & $\begin{array}{l}0.879 \\
(0.450)\end{array}$ \\
\hline$(0.5,0)$ & $\begin{array}{l}0.912 \\
(0.455)\end{array}$ & $\begin{array}{l}0.884 \\
(0.588)\end{array}$ & $\begin{array}{l}0.841 \\
(0.638)\end{array}$ & $\begin{array}{l}0.913 \\
(0.341)\end{array}$ & $\begin{array}{l}0.882 \\
(0.446)\end{array}$ & $\begin{array}{l}0.851 \\
(0.484)\end{array}$ \\
\hline$(0.7,0)$ & $\begin{array}{l}0.886 \\
(0.423)\end{array}$ & $\begin{array}{l}0.834 \\
(0.544)\end{array}$ & $\begin{array}{l}0.796 \\
(0.590)\end{array}$ & $\begin{array}{l}0.882 \\
(0.338)\end{array}$ & $\begin{array}{l}0.830 \\
(0.441)\end{array}$ & $\begin{array}{l}0.787 \\
(0.479)\end{array}$ \\
\hline$(-0.1,0)$ & $\begin{array}{l}0.937 \\
(0.367)\end{array}$ & $\begin{array}{l}0.911 \\
(0.477)\end{array}$ & $\begin{array}{l}0.900 \\
(0.516)\end{array}$ & $\begin{array}{l}0.946 \\
(0.248)\end{array}$ & $\begin{array}{l}0.921 \\
(0.326)\end{array}$ & $\begin{array}{l}0.923 \\
(0.353)\end{array}$ \\
\hline$(-0.3,0)$ & $\begin{array}{l}0.945 \\
(0.317)\end{array}$ & $\begin{array}{l}0.932 \\
(0.413)\end{array}$ & $\begin{array}{l}0.936 \\
(0.446)\end{array}$ & $\begin{array}{l}0.957 \\
(0.210)\end{array}$ & $\begin{array}{l}0.941 \\
(0.276)\end{array}$ & $\begin{array}{l}0.937 \\
(0.298)\end{array}$ \\
\hline$(0,0.3)$ & $\begin{array}{l}0.930 \\
(0.427)\end{array}$ & $\begin{array}{l}0.907 \\
(0.554)\end{array}$ & $\begin{array}{l}0.883 \\
(0.600)\end{array}$ & $\begin{array}{l}0.934 \\
(0.302)\end{array}$ & $\begin{array}{l}0.907 \\
(0.395)\end{array}$ & $\begin{array}{l}0.885 \\
(0.429)\end{array}$ \\
\hline$(0,0.5)$ & $\begin{array}{l}0.924 \\
(0.414)\end{array}$ & $\begin{array}{l}0.902 \\
(0.537)\end{array}$ & $\begin{array}{l}0.874 \\
(0.582)\end{array}$ & $\begin{array}{l}0.929 \\
(0.295)\end{array}$ & $\begin{array}{l}0.895 \\
(0.387)\end{array}$ & $\begin{array}{l}0.884 \\
(0.420)\end{array}$ \\
\hline \multirow[t]{2}{*}{$(0.5,0.5)$} & $\begin{array}{l}0.898 \\
(0.845)\end{array}$ & $\begin{array}{l}0.857 \\
(1.092)\end{array}$ & $\begin{array}{l}0.795 \\
(1.184)\end{array}$ & $\begin{array}{l}0.891 \\
(0.629)\end{array}$ & $\begin{array}{l}0.837 \\
(0.824)\end{array}$ & $\begin{array}{l}0.780 \\
(0.893)\end{array}$ \\
\hline & \multicolumn{3}{|c|}{$h=0.06$} & \multicolumn{3}{|c|}{$h=0.08$} \\
\hline (0.1, & $\begin{array}{l}0.938 \\
(0.234)\end{array}$ & $\begin{array}{l}0.909 \\
(0.308)\end{array}$ & $\begin{array}{l}0.893 \\
(0.333)\end{array}$ & $\begin{array}{l}0.937 \\
(0.205)\end{array}$ & $\begin{array}{l}0.903 \\
(0.271)\end{array}$ & $\begin{array}{l}0.903 \\
(0.290)\end{array}$ \\
\hline$(0.3,0)$ & $\begin{array}{l}0.929 \\
(0.262)\end{array}$ & $\begin{array}{l}0.890 \\
(0.345)\end{array}$ & $\begin{array}{l}0.873 \\
(0.373)\end{array}$ & $\begin{array}{l}0.928 \\
(0.230)\end{array}$ & $\begin{array}{l}0.893 \\
(0.305)\end{array}$ & $\begin{array}{l}0.882 \\
(0.325)\end{array}$ \\
\hline$(0.5,0)$ & $\begin{array}{l}0.909 \\
(0.284)\end{array}$ & $\begin{array}{l}0.877 \\
(0.373)\end{array}$ & $\begin{array}{l}0.846 \\
(0.404)\end{array}$ & $\begin{array}{l}0.908 \\
(0.250)\end{array}$ & $\begin{array}{l}0.869 \\
(0.330)\end{array}$ & $\begin{array}{l}0.850 \\
(0.353)\end{array}$ \\
\hline$(0.7,0)$ & $\begin{array}{l}0.876 \\
(0.284)\end{array}$ & $\begin{array}{l}0.801 \\
(0.373)\end{array}$ & $\begin{array}{l}0.772 \\
(0.403)\end{array}$ & $\begin{array}{l}0.870 \\
(0.251)\end{array}$ & $\begin{array}{l}0.792 \\
(0.333)\end{array}$ & $\begin{array}{l}0.759 \\
(0.355)\end{array}$ \\
\hline$(-0.1,0)$ & $\begin{array}{l}0.947 \\
(0.203)\end{array}$ & $\begin{array}{l}0.918 \\
(0.268)\end{array}$ & $\begin{array}{l}0.916 \\
(0.289)\end{array}$ & $\begin{array}{l}0.947 \\
(0.178)\end{array}$ & $\begin{array}{l}0.917 \\
(0.236)\end{array}$ & $\begin{array}{l}0.916 \\
(0.252)\end{array}$ \\
\hline$(-0.3,0)$ & $\begin{array}{l}0.960 \\
(0.172)\end{array}$ & $\begin{array}{l}0.938 \\
(0.226)\end{array}$ & $\begin{array}{l}0.942 \\
(0.244)\end{array}$ & $\begin{array}{l}0.963 \\
(0.151)\end{array}$ & $\begin{array}{l}0.942 \\
(0.200)\end{array}$ & $\begin{array}{l}0.942 \\
(0.213)\end{array}$ \\
\hline$(0,0.3)$ & $\begin{array}{l}0.935 \\
(0.248)\end{array}$ & $\begin{array}{l}0.898 \\
(0.327)\end{array}$ & $\begin{array}{l}0.887 \\
(0.354)\end{array}$ & $\begin{array}{l}0.934 \\
(0.217)\end{array}$ & $\begin{array}{l}0.894 \\
(0.288)\end{array}$ & $\begin{array}{l}0.886 \\
(0.307)\end{array}$ \\
\hline$(0,0.5)$ & $\begin{array}{l}0.929 \\
(0.243)\end{array}$ & $\begin{array}{l}0.874 \\
(0.320)\end{array}$ & $\begin{array}{l}0.868 \\
(0.346)\end{array}$ & $\begin{array}{l}0.929 \\
(0.213)\end{array}$ & $\begin{array}{l}0.881 \\
(0.283)\end{array}$ & $\begin{array}{l}0.877 \\
(0.302)\end{array}$ \\
\hline$(0.5,0.5)$ & $\begin{array}{l}0.883 \\
(0.520)\end{array}$ & $\begin{array}{l}0.812 \\
(0.684)\end{array}$ & $\begin{array}{l}0.773 \\
(0.739)\end{array}$ & $\begin{array}{l}0.877 \\
(0.455)\end{array}$ & $\begin{array}{l}0.785 \\
(0.602)\end{array}$ & $\begin{array}{l}0.762 \\
(0.642)\end{array}$ \\
\hline
\end{tabular}

Table 4.18: Sieve bootstrap coverage probabilities for $\alpha$ 


\begin{tabular}{|c|c|c|c|c|c|c|}
\hline$(\phi, \psi)$ & $\mathrm{W}$ & $G_{s u b}$ & $G$ & pw & $G_{s u b}$ & $G$ \\
\hline & \multicolumn{3}{|c|}{$h=0.02$} & \multicolumn{3}{|c|}{$h=0.04$} \\
\hline$(0.1,0)$ & $\begin{array}{l}0.950 \\
(0.453)\end{array}$ & $\begin{array}{l}0.945 \\
(0.588)\end{array}$ & $\begin{array}{l}0.935 \\
(0.636)\end{array}$ & $\begin{array}{l}0.956 \\
(0.299)\end{array}$ & $\begin{array}{l}0.950 \\
(0.391)\end{array}$ & $\begin{array}{l}0.937 \\
(0.423)\end{array}$ \\
\hline$(0.3,0)$ & $\begin{array}{l}0.950 \\
(0.406)\end{array}$ & $\begin{array}{l}0.929 \\
(0.528)\end{array}$ & $\begin{array}{l}0.926 \\
(0.630)\end{array}$ & $\begin{array}{l}0.953 \\
(0.281)\end{array}$ & $\begin{array}{l}0.934 \\
(0.368)\end{array}$ & $\begin{array}{l}0.936 \\
(0.398)\end{array}$ \\
\hline$(0.5,0)$ & $\begin{array}{l}0.954 \\
(0.334)\end{array}$ & $\begin{array}{l}0.947 \\
(0.434)\end{array}$ & $\begin{array}{l}0.939 \\
(0.469)\end{array}$ & $\begin{array}{l}0.959 \\
(0.247)\end{array}$ & $\begin{array}{l}0.963 \\
(0.323)\end{array}$ & $\begin{array}{l}0.956 \\
(0.350)\end{array}$ \\
\hline$(0.7,0)$ & $\begin{array}{l}0.970 \\
(0.234)\end{array}$ & $\begin{array}{l}0.967 \\
(0.306)\end{array}$ & $\begin{array}{l}0.964 \\
(0.331)\end{array}$ & $\begin{array}{l}0.974 \\
(0.190)\end{array}$ & $\begin{array}{l}0.973 \\
(0.249)\end{array}$ & $\begin{array}{l}0.974 \\
(0.270)\end{array}$ \\
\hline$(-0.1,0)$ & $\begin{array}{l}0.949 \\
(0.472)\end{array}$ & $\begin{array}{l}0.948 \\
(0.612)\end{array}$ & $\begin{array}{l}0.930 \\
(0.663)\end{array}$ & $\begin{array}{l}0.957 \\
(0.301)\end{array}$ & $\begin{array}{l}0.953 \\
(0.393)\end{array}$ & $\begin{array}{l}0.940 \\
(0.426)\end{array}$ \\
\hline$(-0.3,0)$ & $\begin{array}{l}0.948 \\
(0.465)\end{array}$ & $\begin{array}{l}0.932 \\
(0.604)\end{array}$ & $\begin{array}{l}0.934 \\
(0.653)\end{array}$ & $\begin{array}{l}0.955 \\
(0.290)\end{array}$ & $\begin{array}{l}0.941 \\
(0.379)\end{array}$ & $\begin{array}{l}0.936 \\
(0.411)\end{array}$ \\
\hline$(0,0.3)$ & $\begin{array}{l}0.951 \\
(0.418)\end{array}$ & $\begin{array}{l}0.942 \\
(0.543)\end{array}$ & $\begin{array}{l}0.927 \\
(0.587)\end{array}$ & $\begin{array}{l}0.954 \\
(0.288)\end{array}$ & $\begin{array}{l}0.942 \\
(0.376)\end{array}$ & $\begin{array}{l}0.941 \\
(0.407)\end{array}$ \\
\hline$(0,0.5)$ & $\begin{array}{l}0.954 \\
(0.381)\end{array}$ & $\begin{array}{l}0.932 \\
(0.494)\end{array}$ & $\begin{array}{l}0.929 \\
(0.534)\end{array}$ & $\begin{array}{l}0.957 \\
(0.270)\end{array}$ & $\begin{array}{l}0.943 \\
(0.353)\end{array}$ & $\begin{array}{l}0.940 \\
(0.382)\end{array}$ \\
\hline \multirow[t]{2}{*}{$(0.5,0.5)$} & $\begin{array}{l}0.953 \\
(0.493)\end{array}$ & $\begin{array}{l}0.949 \\
(0.642)\end{array}$ & $\begin{array}{l}0.931 \\
(0.694)\end{array}$ & $\begin{array}{l}0.950 \\
(0.394)\end{array}$ & $\begin{array}{l}0.948 \\
(0.517)\end{array}$ & $\begin{array}{l}0.933 \\
(0.559)\end{array}$ \\
\hline & \multicolumn{3}{|c|}{$h=0.06$} & \multicolumn{3}{|c|}{$h=0.08$} \\
\hline$(0.1,0)$ & $\begin{array}{l}0.958 \\
(0.243)\end{array}$ & $\begin{array}{l}0.946 \\
(0.319)\end{array}$ & $\begin{array}{l}0.932 \\
(0.345)\end{array}$ & $\begin{array}{l}0.955 \\
(0.212)\end{array}$ & $\begin{array}{l}0.944 \\
(0.280)\end{array}$ & $\begin{array}{l}0.933 \\
(0.298)\end{array}$ \\
\hline$(0.3,0)$ & $\begin{array}{l}0.955 \\
(0.232)\end{array}$ & $\begin{array}{l}0.933 \\
(0.305)\end{array}$ & $\begin{array}{l}0.936 \\
(0.329)\end{array}$ & $\begin{array}{l}0.950 \\
(0.204)\end{array}$ & $\begin{array}{l}0.932 \\
(0.270)\end{array}$ & $\begin{array}{l}0.921 \\
(0.287)\end{array}$ \\
\hline$(0.5,0)$ & $\begin{array}{l}0.962 \\
(0.208)\end{array}$ & $\begin{array}{l}0.960 \\
(0.273)\end{array}$ & $\begin{array}{l}0.952 \\
(0.295)\end{array}$ & $\begin{array}{l}0.957 \\
(0.186)\end{array}$ & $\begin{array}{l}0.953 \\
(0.246)\end{array}$ & $\begin{array}{l}0.939 \\
(0.262)\end{array}$ \\
\hline$(0.7,0)$ & $\begin{array}{l}0.972 \\
(0.168)\end{array}$ & $\begin{array}{l}0.981 \\
(0.222)\end{array}$ & $\begin{array}{l}0.965 \\
(0.239)\end{array}$ & $\begin{array}{l}0.965 \\
(0.154)\end{array}$ & $\begin{array}{l}0.973 \\
(0.204)\end{array}$ & $\begin{array}{l}0.957 \\
(0.217)\end{array}$ \\
\hline$(-0.1,0)$ & $\begin{array}{l}0.959 \\
(0.243)\end{array}$ & $\begin{array}{l}0.948 \\
(0.318)\end{array}$ & $\begin{array}{l}0.939 \\
(0.344)\end{array}$ & $\begin{array}{l}0.956 \\
(0.211)\end{array}$ & $\begin{array}{l}0.949 \\
(0.278)\end{array}$ & $\begin{array}{l}0.931 \\
(0.296)\end{array}$ \\
\hline$(-0.3,0)$ & $\begin{array}{l}0.957 \\
(0.232)\end{array}$ & $\begin{array}{l}0.951 \\
(0.305)\end{array}$ & $\begin{array}{l}0.934 \\
(0.329)\end{array}$ & $\begin{array}{l}0.954 \\
(0.201)\end{array}$ & $\begin{array}{l}0.949 \\
(0.265)\end{array}$ & $\begin{array}{l}0.936 \\
(0.282)\end{array}$ \\
\hline$(0,0.3)$ & $\begin{array}{l}0.955 \\
(0.236)\end{array}$ & $\begin{array}{l}0.955 \\
(0.310)\end{array}$ & $\begin{array}{l}0.931 \\
(0.334)\end{array}$ & $\begin{array}{l}0.954 \\
(0.206)\end{array}$ & $\begin{array}{l}0.937 \\
(0.273)\end{array}$ & $\begin{array}{l}0.931 \\
(0.290)\end{array}$ \\
\hline$(0,0.5)$ & $\begin{array}{l}0.956 \\
(0.224)\end{array}$ & $\begin{array}{l}0.951 \\
(0.293)\end{array}$ & $\begin{array}{l}0.938 \\
(0.317)\end{array}$ & $\begin{array}{l}0.954 \\
(0.197)\end{array}$ & $\begin{array}{l}0.939 \\
(0.260)\end{array}$ & $\begin{array}{l}0.931 \\
(0.277)\end{array}$ \\
\hline$(0.5,0.5)$ & $\begin{array}{l}0.950 \\
(0.338)\end{array}$ & $\begin{array}{l}0.947 \\
(0.445)\end{array}$ & $\begin{array}{l}0.934 \\
(0.480)\end{array}$ & $\begin{array}{l}0.945 \\
(0.304)\end{array}$ & $\begin{array}{l}0.942 \\
(0.401)\end{array}$ & $\begin{array}{l}0.922 \\
(0.427)\end{array}$ \\
\hline
\end{tabular}

Table 4.19: Sieve bootstrap coverage probabilities for $\beta$ 


\begin{tabular}{|c|c|c|c|c|}
\hline \multicolumn{5}{|c|}{ Unit root tests } \\
\hline & \multicolumn{2}{|c|}{ ADF test } & \multicolumn{2}{|c|}{ PP test } \\
\hline & $y_{t}$ & $r_{y_{t}}$ & $y_{t}$ & $r_{y_{t}}$ \\
\hline EUA & -0.562 & -14.132 & -0.675 & -17.641 \\
\hline Coal & -2.236 & -12.879 & -2.089 & -16.488 \\
\hline Gas & -1.999 & -13.586 & -2.108 & -18.678 \\
\hline Oil & -1.780 & -13.882 & -1.761 & -18.593 \\
\hline $\mathrm{S}_{50}$ & -3.419 & -15.965 & -4.339 & -19.111 \\
\hline $\mathrm{S}_{600}$ & -3.452 & -15.901 & -4.045 & -19.868 \\
\hline \multirow[t]{5}{*}{ Temp } & -13.054 & - & -15.026 & - \\
\hline & \multicolumn{4}{|c|}{ Critical values: $(90 \%, 95 \%, 99 \%)$} \\
\hline & \multicolumn{2}{|c|}{$(-3.13,-3.42,-3.98)$} & \multicolumn{2}{|c|}{$(-3.13,-3.42,-3.98)$} \\
\hline & \multicolumn{2}{|c|}{ LNV test } & \multicolumn{2}{|c|}{ KPSS test } \\
\hline & $y_{t}$ & $r_{y_{t}}$ & $y_{t}$ & $r_{y_{t}}$ \\
\hline EUA & -1.011 & -14.336 & 1.306 & 0.072 \\
\hline Coal & -2.336 & -13.051 & 0.553 & 0.056 \\
\hline Gas & -2.029 & 13.723 & 0.574 & 0.068 \\
\hline Oil & -2.033 & -13.958 & 1.130 & 0.084 \\
\hline $\mathrm{S}_{50}$ & -3.705 & -16.210 & 0.739 & 0.102 \\
\hline $\mathrm{S}_{600}$ & -3.495 & -16.301 & 0.452 & 0.071 \\
\hline \multirow[t]{3}{*}{ Temp } & -13.147 & - & 0.070 & - \\
\hline & \multicolumn{4}{|c|}{ Critical values: $(90 \%, 95 \%, 99 \%)$} \\
\hline & $(-4.55,-$ & $83,-5.42)$ & $(0.12$, & $14,0.22)$ \\
\hline
\end{tabular}

Table 4.20: Results from the unit root tests of Dickey and Fuller (1979), Phillips and Perron (1988), Leybourne et al. (1998) and Kwiatkowski et al. (1992) 


\begin{tabular}{|c|c|c|c|c|c|c|}
\hline \multicolumn{7}{|c|}{ OLS regression results } \\
\hline & \multicolumn{3}{|c|}{ (d) } & \multicolumn{3}{|c|}{ (e) } \\
\hline & $\boldsymbol{\beta}$ & $s e_{N W}$ & $p$-value & $\boldsymbol{\beta}$ & $s e_{N W}$ & $p$-value \\
\hline Intercept & 0.000 & 0.003 & 0.928 & 0.000 & 0.003 & 0.769 \\
\hline Coal & -0.124 & 0.109 & 0.255 & -0.145 & 0.097 & 0.136 \\
\hline Gas & 0.213 & 0.074 & 0.004 & 0.177 & 0.077 & 0.022 \\
\hline Oil & 0.175 & 0.088 & 0.048 & 0.178 & 0.086 & 0.040 \\
\hline Stoxx 50 & 0.070 & 0.158 & 0.671 & - & - & - \\
\hline Stoxx 600 & - & - & - & 0.112 & 0.165 & 0.498 \\
\hline
\end{tabular}

Table 4.21: Linear regression results additional to the ones presented in Table 4.11. The dependent variable is the return on allowance prices. Here, we consider two new specifications: when adding (d) STOXX 50 or (e) STOXX 600 to the set of explanatory variables in addition to the oil price. As in Table 4.11, the coefficient estimates $(\boldsymbol{\beta})$, the Newey-West standard errors $\left(s e_{N W}\right)$ and the $p$-values are reported. 


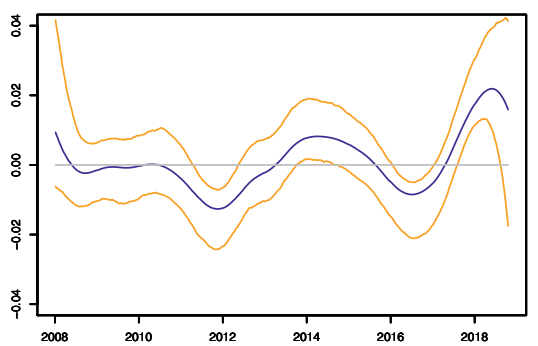

(a) $\hat{\beta}_{0}(t)$

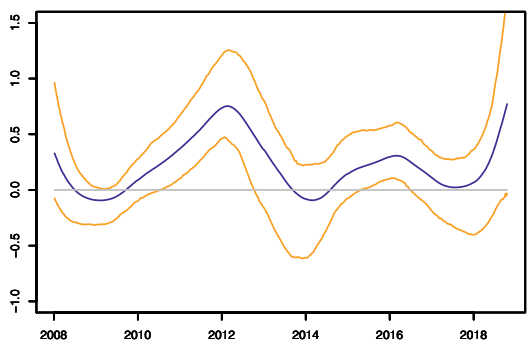

(c) $\hat{\beta}_{\text {gas }}(t)$

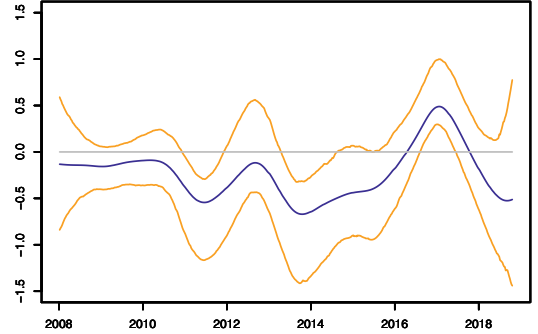

(b) $\hat{\beta}_{\text {coal }}(t)$

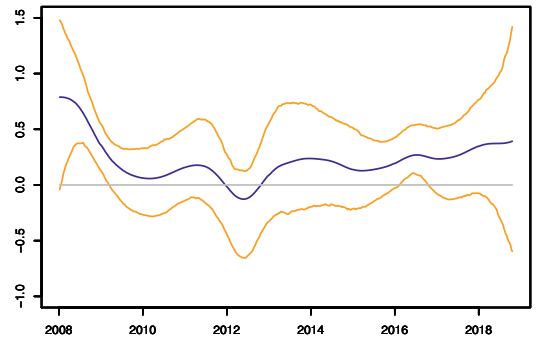

(d) $\hat{\beta}_{\text {oil }}(t)$

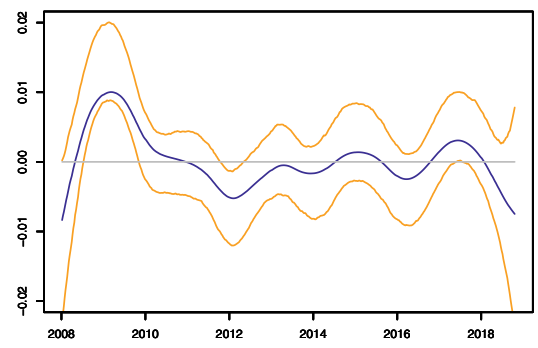

(e) $\hat{\beta}_{t e m p}(t)$

Figure 4.15: Nonparametrically estimated coefficient curves and $95 \%$ confidence intervals $(h=0.05)$ 


\section{Chapter 5}

\section{Conclusion}

At the end of each chapter, a detailed conclusion of the individual contribution has been given. This chapter serves as an overall conclusion of this thesis and discusses directions for further research. Although the chapters are devoted to different topics, they share a common ground. The main similarities are the use of nonparametric estimation methods as well as bootstrap methods to conduct inference and to obtain critical values for testing. This is why Chapter 1 presents a general introduction to both topics providing an overview of some of the related work in both fields.

In Chapter 2, we begin with a simple nonparametric trend model for which we propose a kernel estimator in combination with an autoregressive wild bootstrap. We establish the validity of the bootstrap and investigate a procedure to transform the pointwise confidence intervals into simultaneous confidence bands. We show that these bands provide accurate coverage - both asymptotically as well as in finite samples. The proposed approach works under general conditions which allow for serial correlation, heteroskedasticity as well as missing data. In particular, with the latter aspect the autoregressive wild bootstrap offers an advantage over other frequently used bootstrap methods such as the sieve or the block bootstrap. Missing data are often encountered in environmental applications as the recording of climate data is sensitive to, for instance, instrument failures or adverse measurement conditions.

One prominent application for our approach proposed in Chapter 2 is trend analysis of atmospheric ethane which is presented in Chapter 3. Atmospheric ethane is one of the most abundant hydrocarbon gases in the Earth's atmosphere 
and it is an indicator of air quality and the transport of tropospheric pollution. The recording of observations in this case requires clear skies leading to a severe missing data problem. It is therefore crucial that this is taken into account in the selection of appropriate methods. To confirm previous results which have been obtained in the geophysics literature, we estimate a broken linear trend model before moving on to the nonparametric specification. As a novel result, we obtain confidence intervals around the estimated break location using the autoregressive wild bootstrap.

Going beyond the analysis of ethane data, Chapter 3 can be seen as a collection of trend analysis tools which are motivated by the research questions underlying the ethane application. Based on the nonparametric trend analysis, we propose several tests to further investigate the properties of the estimated trend function. Of particular interest is the existence and location of trend reversals. To this end, we identify locations of extrema in the estimated trend curves and propose a bootstrap method to construct confidence intervals around the locations.

Additionally, we study the shape of the post-reversal trend. In the ethane application, a topic of interest is whether the post-minimum trend is monotonically increasing and if this part of the trend is best described by a linear form. Inspired by these questions, we propose two tests. The first tests if a specific parametric form is appropriate to describe the trend and the second tests if a certain part of the trend function has been monotone.

In Chapter 4, we add a set of exogenous regressors to the nonparametric trend model which adds considerable flexibility and allows us to model complex relationships between variables in a time-varying fashion. Again, we propose a combination of nonparametric kernel estimation and bootstrap methods. The sieve bootstrap performs well in our extensive simulation study. Adding a wild component, however, has not proven to work well in this context. In future research it would be worth investigating how the autoregressive wild bootstrap performs in comparison to the sieve (wild) versions.

The empirical application of Chapter 4 is of interest in environmental economics as we study the price development of CO2 emission allowances in the world's largest cap-and-trade market, the European Emissions Trading Scheme. The price series has been the subject of many studies and political debates since it experienced a long period of low prices against the expectation of market participants. In addition, the abatement-related fundamentals which should be the main price drivers according to economic theory have be shown to have a small 
impact on allowance prices. We find evidence of time-variation of the estimated coefficient curves of some of these price drivers. However, none of the considered drivers can explain the recent upward price movement. To find potential causes of this upward trend is a topic for further research.

From this collection of chapters, several other directions for further research emerge. From Chapters 2 and 3 we observe that, in general, the autoregressive wild bootstrap requires the use of a parameter that determines how much of the dependence of the data is reflected in the bootstrap sample. This parameter is similar to the block length of the block bootstrap. It has to be chosen by the researcher. Although we show that in the context of Chapter 2, the results are not very dependent on this parameter, a data-driven selection method would be useful for practitioners. Such selection methods still need to be developed.

Worth investigating but beyond the scope of this thesis is a thorough analysis of the asymptotic properties of the proposed bootstrap methods of Chapters 3 and 4. These are very promising directions for future research which arise from this thesis. 



\section{Chapter 6}

\section{Valorization}

This dissertation makes a contribution to the academic literature. In addition, it bears value for society which we discuss in the following. In general, the methods proposed in the previous chapters increase the set of available statistical tools to analyze economic and, in particular, environmental developments. In addition, the applications which are presented in this thesis deepen our understanding of important topics in geology and environmental economics. All conclusions drawn from these applications are relevant to the understanding of climate change.

As illustrated in Chapters 2 and 3, we propose a set of trend analysis tools which deepens our understanding of important atmospheric gases. In an application to atmospheric ethane we learn about the presence of a trend reversal pattern which can be associated with the increase of shale gas extraction in the United States. This is an important finding since before this recent rise there were longterm successful measures in place which lead to a steady reduction of the indirect greenhouse gas. With the increased activity in shale gas extraction, these measures were more than offset in less than 10 years.

In addition to being an indirect greenhouse gas and contributing to the formation of ground-level ozone, atmospheric ethane measurements can be used to study another important pollutant which is atmospheric methane. The latter is hard to measure because it is emitted by both anthropogenic and natural sources. Atmospheric ethane, however, is mostly coming from anthropogenic activities which also emit methane in a constant proportion. This is why developments of atmospheric ethane can give us a good indication of the fraction of methane stemming from anthropogenic sources. 
The application of the proposed tools is not limited to the analysis of atmospheric gases. They are valid under very general conditions and can therefore be used for the analysis of various other time series of interest. In order to facilitate the analysis, we make $\mathrm{R}$ code implementing our methods freely available on our websites.

The contribution of Chapter 4 to the analysis of fundamental price drivers in the European Emission Trading Scheme provides a final point worth mentioning in this addendum. The EU ETS is the European Union's flagship policy for regulating carbon dioxide emissions. It currently covers around $45 \%$ of all greenhouse gas emissions in the EU, and it is bound to be expanded as part of the EU's recent Green Deal strategy. Depending on the price of allowances, there is an incentive for firms to engage in short-term mitigation (e.g. fuel switch from coal to gas) and to investment in low-carbon technologies. Economic theory suggests that this is done in a cost-effective way, which is why - in face of more ambitious climate targets and higher policy costs - this policy instrument becomes increasingly important as a tool for society in the presence of climate change.

However, the cost-effectiveness of the EU ETS crucially hinges on efficient price formation, which depends on regulation and policy design as well as the behavior of market participants. In the last years, many concerns have been raised that price formation is inefficient for various reasons, leading to market distortions. If that would indeed be the case, additional measures to correct these distortions are essential to guarantee the proper functioning of the EU ETS. This is why understanding the price development of allowance prices in this scheme is of crucial importance, in particular, after the long period of unexpectedly low prices. In that regard, the results of Chapter 4 suggests that a complementary measure like a carbon price collar could be beneficial to stabilize prices. This recommendation is particularly relevant for the further development of the EU ETS, and thus for the EU's prospects of achieving its ambitious climate targets. 


\section{Bibliography}

Aatola, P., M. Ollikainen, and A. Toppinen (2013). Price determination in the EU ETS market: Theory and econometric analysis with market fundamentals. Energy Economics 36(October 2007), 380-395.

Andrews, D. W. K. (1992). Generic Uniform Convergence. Econometric Theory $8(2), 241-257$.

Bai, J. and P. Perron (1998). Estimating and testing linear models with multiple structural changes. Econometrica 66(1), 47-78.

Billingsley, P. (1968). Convergence of probability measures. Wiley, New York.

Boswijk, H. P. and P. H. Franses (2006). Robust inference on average economic growth. Oxford Bulletin of Economics and Statistics 68, 345-370.

Bühlmann, P. (1997). Sieve bootstrap for time series. Bernoulli 3(2), 123-148.

Bühlmann, P. (1998). Sieve bootstrap for smoothing in nonstationary time series. Annals of Statistics 26, 48-83.

Cai, Z. (2007). Trending time-varying coefficient time series models with serially correlated errors. Journal of Econometrics 136, 163-188.

Caporale, T. (2012). Time-varying CAPM betas and banking sector risks. Economics Letters 115, 293-295.

Cavaliere, G. and A. M. R. Taylor (2008). Bootstrap Unit Root Tests for Time Series with Nonstationary Volatility. Econometric Theory 24(1), 43-71.

Cavaliere, G. and A. M. R. Taylor (2009). Heteroskedastic Time Series with a Unit Root. Econometric Theory 25(5), 1228-1276. 
Chetverikov, D. (2012). Testing regression monotonicity in econometric models. cemmap working paper CWP35/12.

Chow, A. (1960). Tests of equality between sets of coefficients in two linear regressions. Econometrica 28, 591-605.

Chu, C.-K. and J. S. Marron (1991). Comparison of two bandwidths selectors with dependent errors. The Annals of Statistics 19(4), 1906-1918.

Craven, P. and G. Wahba (1978). Smoothing noisy data with spline functions. Numerische Mathematik 31(4), 377-403.

Davidson, J. (2002). Stochastic Limit Theory (2nd edition). Oxford: Oxford University Press.

Davidson, R. and E. Flachaire (2008). The wild bootstrap, tamed at last. Journal of Econometrics 146, 162-169.

De Smedt, I., T. Stavrakou, J.-F. Müller, R. J. van der A, and M. Van Roozendael (2010, sep). Trend detection in satellite observations of formaldehyde tropospheric columns. Geophysical Research Letters 37(18).

Delarue, E., K. Voorspools, and W. D'Haeseleer (2008). Fuel Switching in the Electricity Sector under the EU ETS: Review and Prospective. Journal of Energy Engineering 134(2), 40-46.

Dickey, D. A. and W. A. Fuller (1979). Distribution of the Estimators for Autoregressive Time Series With a Unit Root. Journal of the American Statistical Association $74(366), 427-431$.

Edenhofer, O. (2014, aug). Reforming emissions trading. Nature Climate Change 4(8), 663-664.

Efron, B. (1979). Bootstrap Methods: Another Look at the Jackknife. The Annals of Statistics 7(1), 1-26.

Fan, J. (1992). Design-adaptive nonparametric regression. Journal of the American Statistical Association 87(420), 998-1004.

Fan, J. and I. Gijbels (1996). Local polynomial modeling and its applications. Chapman and Hall, London. 
Franco, B., W. Bader, G. C. Toon, C. Bray, A. Perrin, E. V. Fischer, K. Sudo, C. D. Boone, B. Bovya, B. Lejeune, C. Servais, and E. Mahieu (2015). Retrieval of ethane from ground-based FTIR solar spectra using improved spectroscopy: Recent burden increase above Jungfraujoch. Journal of Quantitative Spectroscopy and Radiative Transfer 160, 36-49.

Franco, B., E. Mahieu, L. K. Emmons, Z. A. Tzompa-Sosa, E. V. Fischer, K. Sudo, B. Bovy, S. Conway, D. Griffin, J. W. Hannigan, K. Strong, and K. A. Walker (2016). Evaluating ethane and methane emissions associated with the development of oil and natural gas extraction in North America. Environmental Research Letters $11(4), 44010$.

Franses, P. H. and T. J. Vogelsang (2005). Are winters getting warmer? Environmental Modeling and Software 20, 1449-1455.

Friedrich, M., E.-M. Mauer, M. Pahle, and O. Tietjen (2019). From fundamentals to financial assets: the evolution of understanding price formation in the EU ETS. Technical report, Kiel, Hamburg.

Friedrich, M., S. Smeekes, and J.-P. Urbain (2019). Autoregressive wild bootstrap inference for nonparametric trends. Journal of Econometrics In Press.

Gardiner, T., A. Forbes, M. de Mazière, C. Vigouroux, E. Mahieu, P. Demoulin, V. Velazco, J. Notholt, T. Blumenstock, F. Hase, I. Kramer, R. Sussmann, W. Stremme, J. Mellqvist, A. Strandberg, K. Ellingsen, and M. Gauss (2008). Trend analysis of greenhouse gases over Europe measuerd by a network of ground-based remote FTIR instruments. Atmospheric Chemistry and Physics 8, 6719-6727.

Gasser, T. and H. G. Müller (1984). Estimating regression functions and their derivatives by the kernel method. Scandinavian Journal of Statistics 11, 171185.

Ghosal, S., A. Sen, and A. van der Vaart (2000). Testing Monotonicity of Regression. Annals of Statistics 28, 1054-1082.

Gine, E. and J. Zinn (1990). Bootstrapping General Empirical Measures. The Annals of Probability 18(2), 851-869.

Gonzalés, A. and T. Teräsvirta (2008). Modelling autoregressive processes with a shifting mean. Studies in Nonlinear Dynamics 8 Econometrics 12(1). 
Gregory, K. B., S. N. Lahiri, and D. J. Nordman (2015, may). A Smooth Block Bootstrap for Statistical Functionals and Time Series. Journal of Time Series Analysis 36(3), 442-461.

Gregory, K. B., S. N. Lahiri, and D. J. Nordman (2018). A smooth block bootstrap for quantile regression with time series. The Annals of Statistics 46(3), 11381166.

Hall, P. and C. C. Heyde (1980). Martingale Limit Theory and Its Application. New York: Springer Academic Press.

Hall, P. and J. Horowitz (2013). A simple bootstrap method for constructing nonparametric confidence bands for functions. The Annals of Statistics 41(4), $1892-1921$.

Hansen, B. E. (1991). Strong Laws for Dependent Heterogeneous Processes. Econometric Theory 7(2), 213-221.

Hansen, B. E. (2009). Econometrics. Univeristy of Wisconsin.

Hardle, W. and J. S. Marron (1991). Bootstrap Simultaneous Error Bars for Nonparametric Regression. The Annals of Statistics 19(2), 778-796.

Hausmann, P., R. Sussmann, and D. Smale (2016, mar). Contribution of oil and natural gas production to renewed increase in atmospheric methane (20072014): top-down estimate from ethane and methane column observations. Atmos. Chem. Phys. 16(5), 3227-3244.

Helmig, D., S. Rossabi, J. Hueber, P. Tans, S. A. Montzka, K. Masarie, K. Thoning, C. Plass-Duelmer, A. Claude, L. J. Carpenter, A. C. Lewis, S. Punjabi, S. Reimann, M. K. Vollmer, R. Steinbrecher, J. W. Hannigan, L. K. Emmons, E. Mahieu, B. Franco, D. Smale, and A. Pozzer (2016, jun). Reversal of global atmospheric ethane and propane trends largely due to US oil and natural gas production. Nature Geoscience 9, 490.

Hidalgo, J. (2005). Uniform confidence bands for kernel regression estimates with dependent data. Unpublished manuscript.

Hintermann, B. (2010). Allowance price drivers in the first phase of the EU ETS. Journal of Environmental Economics and Management 59(1), 43-56. 
Jansson, M. (2002). Consistent covariance matrix estimation for linear processes. Econometric Theory 18, 1449-1459.

Juhrich, K. (2016). CO2 emission factors for fossil fuels. Climate Change, Umweltbundesamt 28, 1-48.

Kapetanios, G. (2008). Bootstrap-based tests for deterministic time-varying coefficients in regression models. Computational Statistics \& Data Analysis 53, $534-545$.

Klein Tank, A. M. G., J. B. Wijngaard, G. P. Können, R. Böhm, G. Demarée, A. Gocheva, M. Mileta, S. Pashiardis, L. Hejkrlik, C. Kern-Hansen, R. Heino, P. Bessemoulin, G. Müller-Westermeier, M. Tzanakou, S. Szalai, T. Pálsdóttir, D. Fitzgerald, S. Rubin, M. Capaldo, M. Maugeri, A. Leitass, A. Bukantis, R. Aberfeld, A. F. V. van Engelen, E. Forland, M. Mietus, F. Coelho, C. Mares, V. Razuvaev, E. Nieplova, T. Cegnar, J. Antonio López, B. Dahlström, A. Moberg, W. Kirchhofer, A. Ceylan, O. Pachaliuk, L. V. Alexander, and P. Petrovic (2002). Daily dataset of 20th-century surface air temperature and precipitation series for the European Climate Assessment. International Journal of Climatology 22(12), 1441-1453.

Koch, N., S. Fuss, G. Grosjean, and O. Edenhofer (2014). Causes of the EU ETS price drop: Recession, CDM, renewable policies or a bit of everything?-New evidence. Energy Policy 73, 676-685.

Kreiss, J.-P. (1992). Bootstrap procedures for AR(1)-processes. In K.H. Jöckel, G. Rothe and W. Sender (Eds.), Bootstrapping and Related Techniques, Number 376 in Lecture Notes in Economics and Mathematical Systems.

Kristensen, D. (2012). Non-parametric detection and estimation of structural change. Econonmetrics Journal 15, 420-461.

Künsch, H. R. (1989). The jackknife and the bootstrap for general stationary observations. pp. 1217-1241.

Kwiatkowski, D., P. C. B. Phillips, P. Schmidt, and Y. Shin (1992). Testing the null hypothesis of stationarity against the alternative of a unit root: How sure are we that economic time series have a unit root? Journal of Econometrics 54(1), 159-178. 
Leucht, A. and M. H. Neumann (2013). Dependent wild bootstrap for degenerate U- and V-statistics. Journal of Multivariate Analysis 117, 257-280.

Leybourne, S., P. Newbold, and D. Vougas (1998). Unit roots and smooth transitions. Journal of Time Series Analysis 19(1), 83-97.

Liang, Z. and Q. Li (2012). Functional coefficient regression models with time trend. Journal of Econometrics 170, 15-31.

Ling, S., W. K. Li, and M. McAleer (2003). Estimation and Testing for Unit Root Processes with GARCH $(1,1)$ Errors: Theory and Monte Carlo Evidence. Econometric Reviews 22(2), 179-202.

Lomb, N. R. (1976, feb). Least-squares frequency analysis of unequally spaced data. Astrophysics and Space Science 39(2), 447-462.

Lutz, B. J., U. Pigorsch, and W. Rotfuß (2013). Nonlinearity in cap-and-trade systems: The EUA price and its fundamentals. Energy Economics 40, 222-232.

Mahieu, E., M. P. Chipperfield, J. Notholt, T. Reddmann, J. Anderson, P. F. Bernath, T. Blumenstock, M. T. Coffey, S. S. Dhomse, W. Feng, B. Franco, L. Froidevaux, D. W. T. Griffith, J. W. Hannigan, F. Hase, R. Hossaini, N. B. Jones, I. Morino, I. Murata, H. Nakajima, M. Palm, C. Paton-Walsh, J. M. R. III, M. Schneider, C. Servais, D. Smale, and K. A. Walker (2014, nov). Recent Northern Hemisphere stratospheric $\mathrm{HCl}$ increase due to atmospheric circulation changes. Nature 515, 104.

McKitrick, R. and T. J. Vogelsang (2014). HAC robust trend comparisons among climate series with possible level shifts. Environmetrics 25(7), 528-547.

McLeish, D. L. (1975). A Maximal Inequality and Dependent Strong Laws. The Annals of Probability 3(5), 829-839.

Nadaraya, E. A. (1964). On estimating regression. Theory Prob. Appl. 9. pp. $141-142$.

Neumann, M. H. (1998). Pointwise confidence intervals in nonparametric regression with heteroskedastic error structure. Statistics 29(1), 1-36.

Neumann, M. H. and J. Polzehl (1998). Simultaneous bootstrap confidence bands in nonparametric regression. Journal of Nonparametric Statistics 9, 307-333. 
Newey, W. K. and K. D. West (1987). A Simple, Positive Semi-Definite, Heteroskedasticity and Autocorrelation Consistent Covariance Matrix. Econometrica 55(3), 703-708.

Norris, J. R., R. J. Allen, A. T. Evan, M. D. Zelinka, C. W. O'Dell, and S. A. Klein (2016). Evidence for climate change in the satellite cloud record. Nature 536, 72-75.

Orbe, S., E. Ferreira, and J. Rodriguez-Poo (2005). Nonparametric estimation of time varying parameters under shape restrictions. Journal of Econometrics $126,53-77$.

Orbe, S., E. Ferreira, and J. Rodriguez-Poo (2006). On the estimation and testing of time varying constraints in econometric models. Statistica Sinica 16, 13131333.

Paparoditis, E. and D. Politis (2002, jun). The tapered block bootstrap for general statistics from stationary sequences. The Econometrics Journal 5(1), 131-148.

Phillips, P. C. B. (2001). Trending time series and macroeconomic activity: some present and future challenges. Journal of Econometrics 100, 21-27.

Phillips, P. C. B. and P. Perron (1988). Testing for a Unit Root in Time Series Regression. Biometrika 75(2), 335-346.

Politis, D. N., J. P. Romano, and M. Wolf (1999). Subsampling. Springer Series in Statistics. New York, NY: Springer New York.

Priestley, M. (1965). Evolutionary spectra and nonstationary processes. Journal of Royal Statistical Society, Series B 27, 204-237.

Rickels, W., D. Görlich, and S. Peterson (2014). Explaining European Emission Allowance Price Dynamics: Evidence from Phase II. German Economic Review 16(2), 181-202.

Robinson, P. M. (1989). Nonparametric estimation of time varying parameters In: Hackl, P. (Ed.), Statistics, Analysis and Forecasting of Economic Structural Change. Springer, Berlin.

Robinson, P. M. (1991). Time varying nonlinear regression In: Hackl, P. (Ed.), Statistics, Analysis and Forecasting of Economic Structural Change. Springer, Berlin. 
Santos, C., D. Hendry, and S. Johansen (2008). Automatic selection of indicators in a fully saturated regression. Computational Statistics 23, 317-335.

Scargle, J. D. (1982, dec). Studies in astronomical time series analysis. II - Statistical aspects of spectral analysis of unevenly spaced data. The Astrophysical Journal 263, 835.

Shao, X. (2010a). Extended tapered block bootstrap. Statistica Sinica 20(2), $807-821$.

Shao, X. (2010b). The dependent wild bootstrap. Journal of the American Statistical Association 105, 218-235.

Simpson, I. J., M. P. S. Andersen, S. Meinardi, L. B. N. J. B. D. Helmig, F. S. Rowland, and D. R. Blake (2012). Longterm decline of global atmospheric ethane concentrations and implications for methane. Nature 488, 490-494.

Smeekes, S. and A. M. R. Taylor (2012). Bootstrap union tests for unit roots in the presence of nonstationary volatility. Econometric Theory 28(2), 422-456.

Smeekes, S. and J.-P. Urbain (2014). A multivariate invariance principle for modified wild bootstrap methods with an application to unit root testing. Maastricht University Research Papers, RM/14/008.

Vinciguerra, T., S. Yao, J. Dadzie, A. Chittams, T. Deskins, S. Ehrman, and R. R. Dickerson (2015). Regional air quality impacts of hydraulic fracturing and shale natural gas activity: evidence from ambient VOC observations. Atmos. Environ. 110, 144-150.

Watson, G. S. (1964). Smooth regression analysis. Sankhyā Ser. A 26, 359-372.

Wu, W. B. (1986). Jackknife, Bootstrap and Other Resampling Methods in Regression Analysis. The Annals of Statistics 14(4), 1261-1295.

Wu, W. B. and Z. Zhao (2007). Inference of trends in time series. Journal of the Royal Statistical Society Series B 69, 391-410.

Yurganov, L. N., T. Blumenstock, E. I. Grechko, F. Hase, E. J. Hyer, I. S. Kasischke, M. Koike, Y. Kondo, I. Kramer, F.-Y. Leung, E. Mahieu, J. Mellqvist, J. Notholt, P. C. Novelli, C. P. Rinsland, H. E. Scheel, A. Schulz, A. Strandberg, R. Sussmann, H. Tanimoto, V. Velazco, R. Zander, and Y. Zhao (2004). A quantitative assessment of the 1998 carbon monoxide emission anomaly in the 
Northern Hemisphere based on total column and surface concentration measurements. Journal of Geophysical Research 109, D15305.

Yurganov, L. N., P. Duchatelet, A. V. Dzhola, D. P. Edwards, F. Hase, I. Kramer, E. Mahieu, J. Mellqvist, J. Notholt, P. C. Novelli, A. Rockmann, H. E. Scheel, M. Schneider, A. Schulz, A. Strandberg, R. Sussmann, H. Tanimoto, V. Velazco, J. R. Drummond, and J. C. Gille (2005). Increased Northern Hemispheric carbon monoxide burden in the troposphere in 2002 and 2003 detected from the ground and from space. Atmospheric Chemistry and Physics 5(2), 563-573.

Zeng, G., S. W. Wood, O. Morgenstern, N. B. Jones, J. Robinson, and D. Smale (2012). Trends and variations in CO, C2H6, and HCN in the Southern Hemisphere point to the declining anthropogenic emissions of $\mathrm{CO}$ and $\mathrm{C} 2 \mathrm{H} 6$. Atmospheric Chemistry and Physics 12(16), 7543-7555.

Zhou, Z. and W. B. Wu (2010). Simultaneous inference of linear models with time varying coefficients. Journal of the Royal Statistical Society Series B 72, 513-531. 



\section{Nederlandse Samenvatting}

Deze dissertatie betreft de analyse van tijdreeksen data. De nadruk ligt op het onderzoeken van zowel variabelen met een deterministische tijdtrend als relaties tussen variabelen die veranderen door de tijd heen. Daartoe introduceren wij in hoofdstuk 2 en 3 verschillende methodieken om tijdtrends en specifieke eigenschappen van de geschatte trendlijn te onderzoeken. Voor de analyse van de relatie tussen meerdere variabelen, presenteren wij in hoofdstuk 4 een setting waarin zulke relaties kunnen worden gemodelleerd op een flexibele manier met het gebruik van onbekende functies van tijd. In beide gevallen pleiten wij voor het gebruik van niet-parametrische regressie technieken en bootstrap methoden. Wij benadrukken dat de methoden niet alleen kunnen worden toegepast op economische variabelen, maar ook op klimatologische tijdreeksen. De focus van deze dissertatie is met name gericht op laatstgenoemde onderwerp en alle methodologische bijdragen aan de literatuur zijn gecombineerd met toepassingen die relevant zijn voor de studie van klimaatverandering. De volgende alinea's bespreken de bijdragen van elk hoofdstuk in meer detail.

Hoofdstuk 1 bestaat uit een overzicht van de komende hoofdstukken en bevat tevens een eerste discussie over de econometrische en statistische methoden die gebruikt worden in deze thesis. Het doel is om een algemene introductie te verschaffen voor de volgende twee onderwerpen: de niet-parametrische regressie schatting en het opstellen van betrouwbaarheidsintervallen met behulp van de bootstrap. We bespreken in dit hoofdstuk voornamelijk de elementaire intuïtie achter trend schatting door middel van lokaal-constante alsmede lokaal-lineaire kernel smoothers. Wij introduceren tevens het eerste bootstrap algoritme voor 
het opstellen van betrouwbaarheidsintervallen rondom een geschatte, van belang zijnde parameter.

In hoofdstuk 2 is het startpunt een algemeen deterministisch trend model waarin de trend een onbekende functie van tijd is. De niet-parametrische schattingsmethode van dit model is een lokaal-constante kernel schatter. De voornaamste bijdrage van dit hoofdstuk aan de bestaande literatuur is de bootstrap methode in combinatie met een techniek die betrouwbaarheidsintervallen in betrouwbaarheidsbanden transformeert, welke gelijktijdig geldig zijn over meerdere tijdspunten. We tonen de geldigheid van de bootstrap methode aan onder algemene voorwaarden, die de aanwezigheid van seriële correlatie, heteroscedasticiteit en ontbrekende data toestaan.

De empirische toepassing in hoofdstuk 2 en het voornaamste thema in hoofdstuk 3 bestaan uit tijdtrends in atmosferisch ethaan, een belangrijke atmosferische vervuiler en indirect broeikasgas. Wij bestuderen tijdreeksen van ethaan metingen verkregen van zes verschillende stations verspreid over de hele wereld. We passen de niet-parametrische trend methode van hoofdstuk 2 toe en introduceren een set van technieken voor inferentie die toegepast kunnen worden om meer te leren over de trend schatting. Bovendien beschouwen wij een parametrische benadering bestaande uit een lineaire trend met onderbreking.

Hoofdstuk 4 bevat de laatste bijdrage aan deze thesis. Het bestaat uit een flexibele methodiek om (economische) verbanden te bestuderen die onderhevig zijn aan structurele veranderingen. Vergeleken met de modellen in de vorige hoofdstukken staat dit nieuwe model de gebruiker toe om niet alleen een tijdtrend maar ook exogene variabelen toe te voegen. De coëfficiënten van deze onafhankelijke variabelen zijn niet constant - zoals in het lineaire regressie model - maar tijdsafhankelijk. De vorm van verandering hoeft niet te worden gespecificeerd door de gebruiker, maar wordt geschat en is dus afgeleid van de data. Dit is de reden waarom dit model grote flexibiliteit biedt. Ons voorstel is het gebruik van een niet-parametrische lokaal-lineaire kernel schatter in combinatie met een sieve bootstrap om betrouwbaarheidsintervallen te verkrijgen. In een Monte Carlo simulatie studie tonen wij aan dat de methode goed werkt in kleine steekproeven, ook in de aanwezigheid van serieel-gecorreleerde storingstermen. Net als in hoofdstuk 2 onderzoeken wij het algoritme om gelijktijdige betrouwbaarheidsbanden op te stellen en concluderen dat deze accurate dekkingswaarschijnlijkheid hebben in de simulatie studie. 
In het empirische gedeelte van hoofdstuk 4 passen wij de voorgestelde aanpak toe om het verband tussen de prijs van emissierechten in de EU en hun fundamentele prijsaandrijvers te bestuderen. Op basis van eerdere literatuur wordt er verwacht dat deze relatie niet constant is door de tijd heen. Wij tonen aan dat er inderdaad een significante tijdvariatie aanwezig is in de geschatte coëfficiënten. 



\section{Curriculum Vitae}

Marina Friedrich was born on 1 June, 1987 in Geilenkirchen, Germany. She attended high school between 1997 and 2006 at Kreisgymnasium Heinsberg, where she received her Gymnasium diploma. Subsequently, she participated in an apprenticeship program offered by Kreissparkasse Heinsberg to become a certified bank clerk, where she worked for 3 years. After 2.5 of these 3 years she obtained the desired qualification and continued to work for 6 months at Kreissparkasse Heinsberg. In September 2009, she started her undergraduate studies in Econometrics and Operations Research at Maastricht University, during which she spent one semester at University of California in Berkeley, USA, as a visiting student. As a result, she obtained her B.Sc. degree in August 2012 with distinction (cum laude). In August 2014, she obtained her M.Sc. degree in Economic and Financial Research with distinction (cum laude) from Maastricht University.

After graduation, Marina joined the Department of Quantitative Economics as a Ph.D. candidate in September 2014, under the supervision of Prof. dr. JeanPierre Urbain and Dr. Stephan Smeekes. In October 2016, Dr. Eric Beutner and Prof. dr. Franz Palm became involved in the supervision. The results of her research are presented in this thesis.

Marina spent five weeks at Oxford University as visiting researcher in November 2016 and she presented her work at various international conferences, such as the International Conference on Computational and Financial Econometrics (CFE) in Pisa and London, the Netherlands Econometric Study Group (NESG) meeting in Maastricht, the European Meeting of Statisticians in Amsterdam, the conference on Econometric Models of Climate Change (EMCC) in Aarhus and Frascati as well as the Maastricht Workshop on Advances in Quantitative Economics II. 
From February 2018 to May 2020, Marina was part of the Potsdam Institute of Climate Impact Research in their research domain on Mitigation Pathways and since August 2020, she is an Assistant Professor of Econometrics at Vrije Universiteit Amsterdam. 


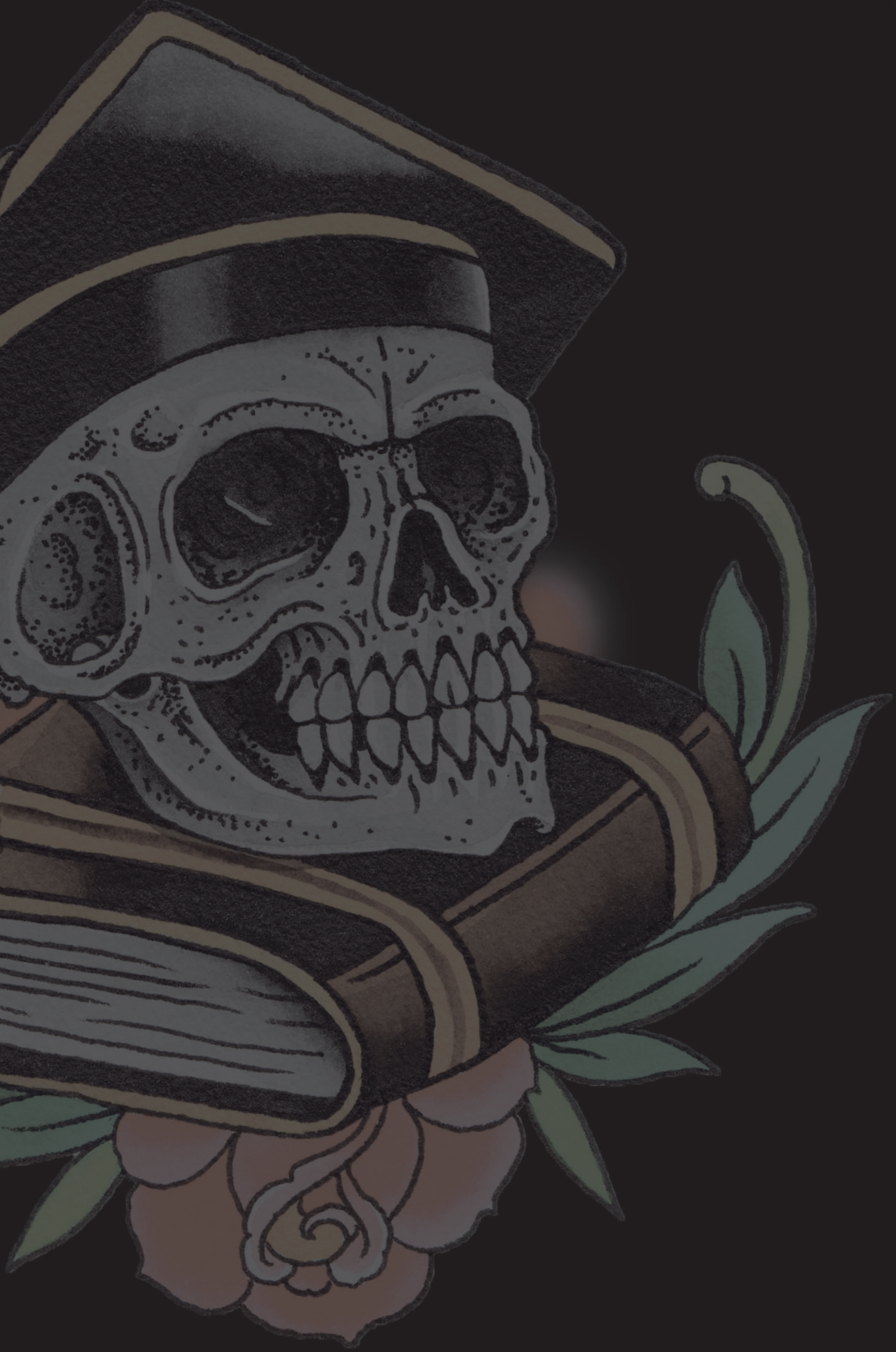

